\title{
Gauge and Lorentz invariant one-pion exchange currents in electron scattering from a relativistic Fermi gas
}

\author{
J.E. Amaro ${ }^{\mathrm{a}, *}$, M.B. Barbaro $^{\mathrm{b}}$, J.A. Caballero ${ }^{\mathrm{c}}$, T.W. Donnelly ${ }^{\mathrm{d}}$, A. Molinari ${ }^{\mathrm{b}}$ \\ ${ }^{a}$ Departamento de Física Moderna, Universidad de Granada, Campus de Fuentenueva, E-18071 Granada, Spain \\ ${ }^{\mathrm{b}}$ Dipartimento di Fisica Teorica, Università di Torino and INFN, Sezione di Torino Via P. Giuria 1, 10125 Torino, Italy \\ ${ }^{\mathrm{c}}$ Departamento de Física Atómica, Molecular y Nuclear, Universidad de Sevilla, Apdo. 1065, E-41080 Sevilla, Spain \\ ${ }^{\mathrm{d}}$ Center for Theoretical Physics, Laboratory for Nuclear Science and Department of Physics, \\ Massachusetts Institute of Technology, Cambridge, MA 02139, USA
}

Received 1 April 2002

editor: G.E. Brown

\begin{abstract}
A consistent analysis of relativistic pionic correlations and meson-exchange currents for electroweak quasielastic electron scattering from nuclei is carried out. Fully relativistic one-pion-exchange electromagnetic operators are developed for use in one-particle emission electronuclear reactions within the context of the relativistic Fermi gas model. Then the exchange and pionic correlation currents are set up fully respecting the gauge invariance of the theory. Emphasis is placed on the self-energy current which, being infinite, needs to be renormalized. This is achieved starting in the Hartree-Fock framework and then expanding the Hartree-Fock current to first order in the square of the pion coupling constant to obtain a truly gauge invariant, one-pion-exchange current. The model is applied to the calculation of the parity-conserving and parity-violating inclusive responses of nuclei. Interestingly, in the pionic correlations terms exist which arise uniquely from relativity, although their impact on the responses is found to be modest. (c) 2002 Elsevier Science B.V. All rights reserved.
\end{abstract}

PACS: 25.30.Rw; 14.20.Gk; 24.10.Jv; 24.30.Gd; 13.40.Gp

Keywords: Nuclear reactions; Inclusive electron scattering; Pionic correlations; Meson-exchange currents; Relativistic Fermi gas

\section{Contents}

1. Introduction

\footnotetext{
${ }^{*}$ Corresponding author. Tel.: +34-958-246-172; fax: 34-958-249-487.

E-mail address: amaro@ugr.es (J.E. Amaro).
} 


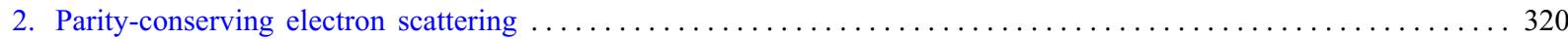

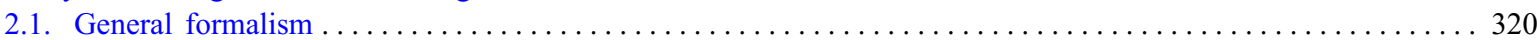

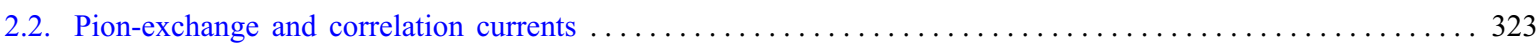

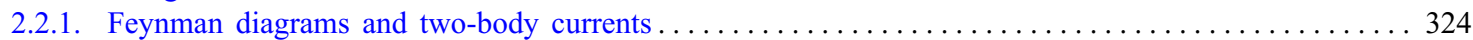

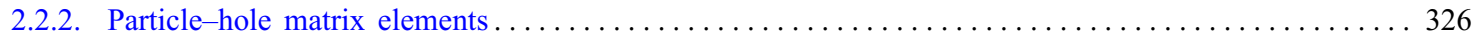

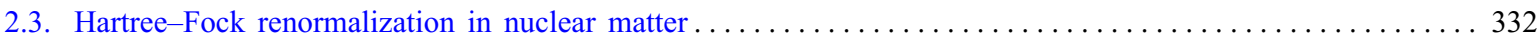

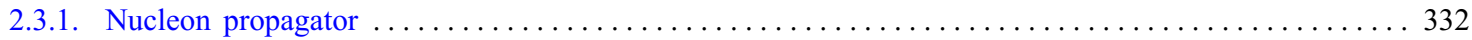

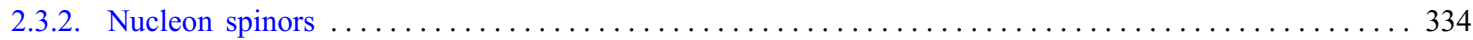

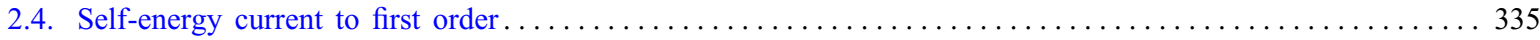

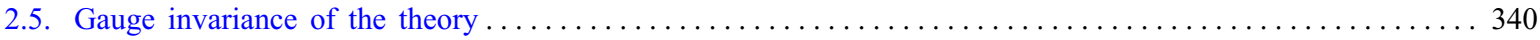

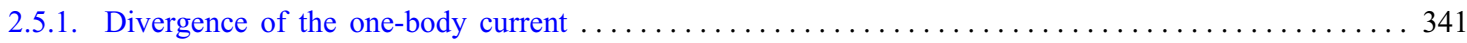

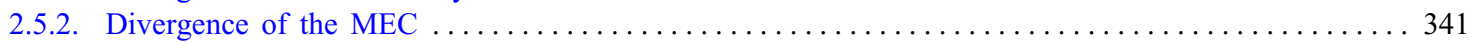

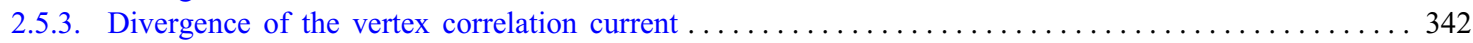

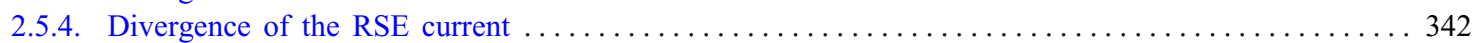

2.6. Nuclear hadronic tensor and electromagnetic response functions $\ldots \ldots \ldots \ldots \ldots \ldots \ldots \ldots \ldots \ldots \ldots \ldots \ldots \ldots \ldots$

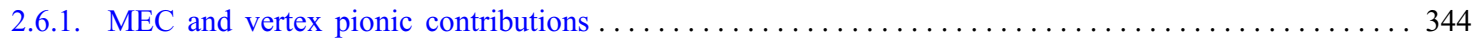

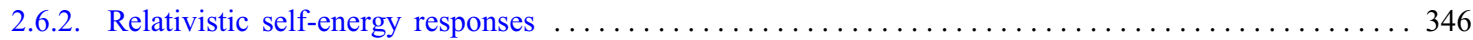

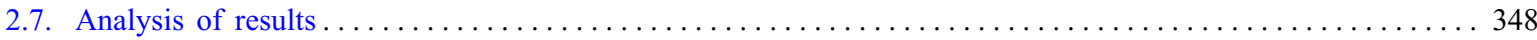

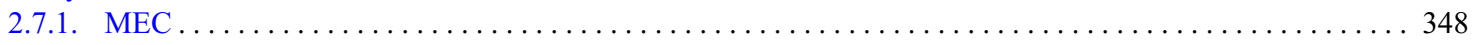

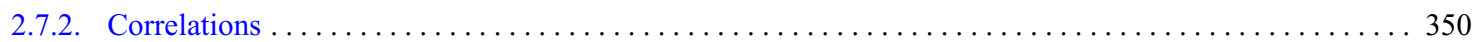

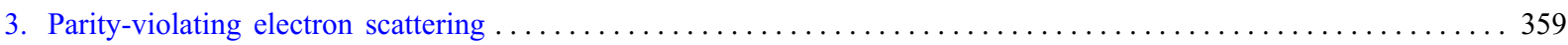

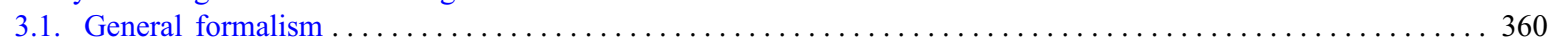

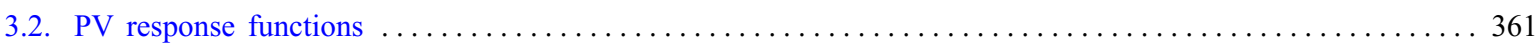

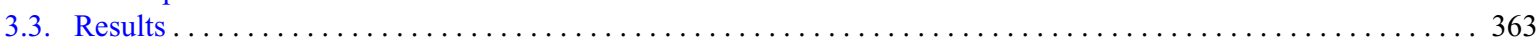

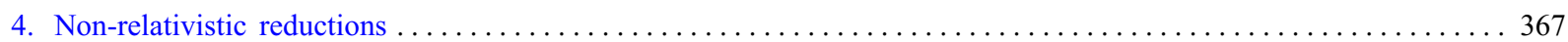

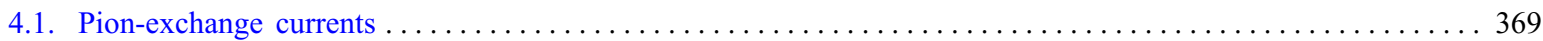

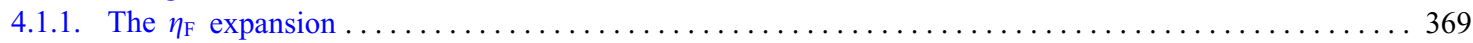

4.1.2. Results for the responses using the relativized MEC model ......................... 375

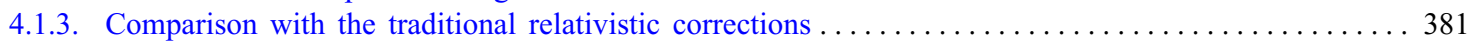

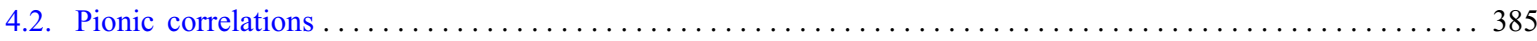

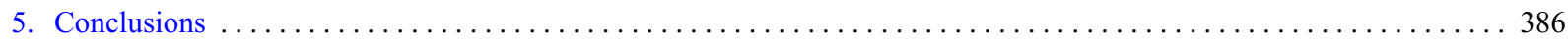

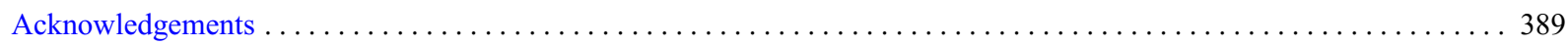

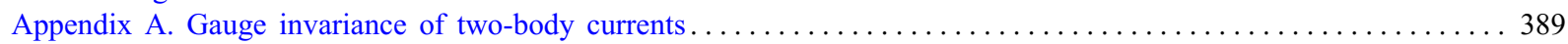

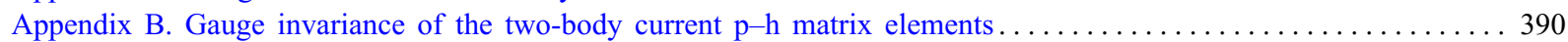

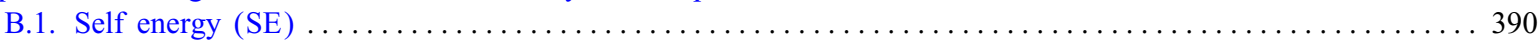

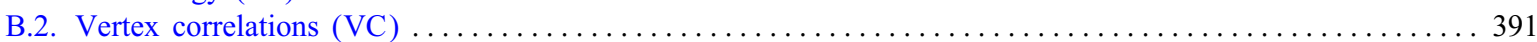

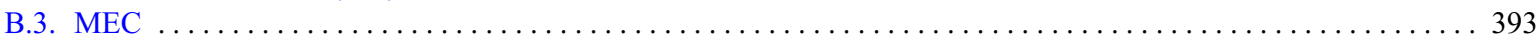

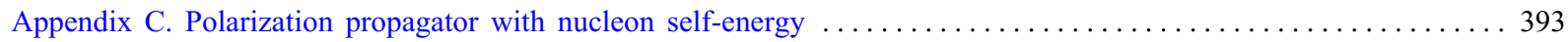

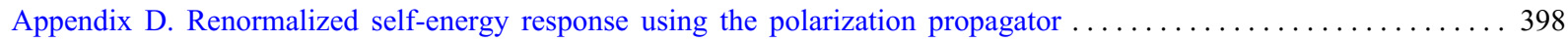

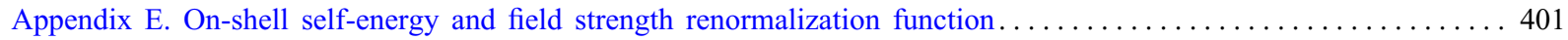

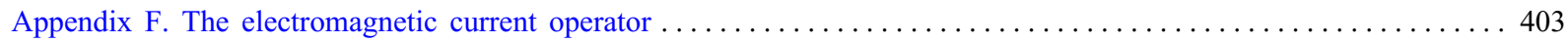

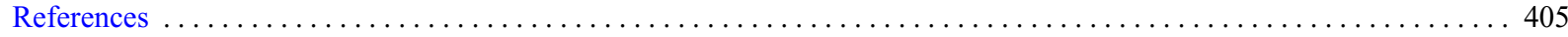

\section{Introduction}

In modern experimental studies of electron scattering from nuclei [1-3], the typical values of energy and momentum transfer are comparable to or even larger than the mass scale set by the nucleon mass and accordingly one must expect relativistic effects to be important. Unfortunately, the wave functions and operators used to describe this high-energy regime have been treated only 
approximately. Indeed it is still the case that many calculations continue to be done at the nonrelativistic level with leading-order expansions of the electroweak currents involved [4-8]. However, a number of studies in recent years show that such an approach is highly constrained to work only at relatively low energies and momenta.

In order to gain insight into which ingredients can or cannot be non-relativistically approximated, we have employed a simple model in which Lorentz covariance and gauge invariance can be maintained, namely, the relativistic Fermi gas model (RFG). Since our focus is placed on the quasielastic region where high-energy knockout of nucleons is kinematically favored, we believe that this model, while undoubtedly too simple to encompass all aspects of nuclear dynamics is nevertheless a convenient place to start in such explorations. Indeed, the problem of relativity in electroweak studies of nuclei is so difficult [9-18] that only in special frameworks such as the RFG can we hope to carry out all but rather severely approximated modeling.

In the quasielastic regime we also expect pions to play a role that differs from the dynamics typically occurring near the Fermi surface where one expects other mesons ( $\sigma$ and $\omega$ in particular) to dominate. For quasielastic scattering the residual interaction of relevance is principally that between a low-energy hole and a very high-energy particle, and for this the pion is expected to play an important role. Accordingly, as the next step after the basic relativistic Fermi gas of non-interacting nucleons we have concentrated on one-pion-exchange (OPE) effects in our description of the nuclear responses. These occur as correlation effects and also as two-body meson-exchange current effects. After developing approximate methods for modeling to this order, in recent work we have reached the stage where large classes of effects can be incorporated fully relativistically. The present paper is a comprehensive discussion of what we have learned to this point based on this type of approach, together with comments on what directions future studies could follow.

In particular, in a recent paper [19] we investigated the role played by pions in inclusive electron scattering from nuclei within the context of one-particle one-hole (1p-1h) excitations, i.e., for the dominant modes in the quasielastic regime. There we extended our previous work [20,21] where a systematic investigation of relativistic effects in the nuclear electromagnetic responses spanning a wide range of kinematical conditions and accounting for both meson-exchange and isobar currents was carried out. In these studies a consistent first-order operator, embodying all Feynman diagrams built out of nucleons and pions with one exchanged pion and one photon attached to all the possible lines was set up to represent the two-body current. Importantly, the latter has been explicitly proven to be gauge invariant in [19].

In addition to the usual contact and pion-in-flight meson-exchange currents (MEC), this fully relativistic operator includes as well the so-called correlation currents. The latter are often not included in model calculations because they give rise to contributions assumed already to be accounted for (at least in part) in the initial and final nuclear wave functions [22,23]. However, our model is based on an uncorrelated relativistic Fermi gas whose states are Slater determinants built out of (Dirac) plane waves. Within a perturbative approach, we are free to consider the one-pion correlation contributions to the responses as arising either explicitly in the wave functions or from an appropriate current operator acting on unperturbed states: our choice has been the latter. Clearly, should it be possible to sum up the whole perturbative expansion, then the results obtained starting with the true "correlated" wave function would be exactly recovered.

In this paper we provide a deeper analysis of the impact of pions on the nuclear electromagnetic response in the $1 \mathrm{p}-1 \mathrm{~h}$ channel [24-28]. Just as for the MEC, the two-body correlation current 
also contributes in this sector and, of course, it should do so consistently, namely fulfilling gauge invariance at the level of OPE.

When the operator associated with a two-body current acts on the RFG ground state, in general, it changes the quantum numbers of two nucleons: the $1 \mathrm{p}-1 \mathrm{~h}$ matrix element is then obtained via the integration of a one-particle state over the Fermi sea. In the case of the correlation current two contributions are thus obtained. The first one, sometimes referred to as a vertex correction [29], arises from the exchange of a pion between the particle and hole; the second relates to the Fock self-energy (SE) [29,30] and dresses the particle and hole propagation lines. This one diverges, since it corresponds to an SE insertion on an external line, which field theory [31,32] tells us not to include in a perturbative expansion. Instead, one should apply a renormalization procedure to dress the external lines by summing up the entire perturbative series of self-energy insertions. In the nuclear context, this procedure leads to the relativistic Hartree-Fock (HF) approach.

In some relativistic calculations [29,30], this contribution has been treated by introducing from the outset an HF propagator in the medium, which accounts for the SE diagrams. In-medium form factors for the $1 \mathrm{p}-1 \mathrm{~h}$ current were also introduced neglecting however any momentum dependence in the self-energy and effective mass. Thus in [30] the self-consistent Hartree mean field was inserted into the single-particle propagator, automatically including the Pauli blocking of $N \bar{N}$ pairs, whose contribution was thus included in the random-phase-approximation (RPA) responses computed there. A similar semi-phenomenological treatment of the nucleon self-energy in the medium at the non-relativistic level was implemented in [4]. More recently, the Dirac structure of nucleon self-energy in nuclear matter has been studied in [33], while a finite nuclei calculation based on the $\sigma-\omega$ model can be found in [9], where the relativistic Hartree model of [10] is used for the single-particle bound states.

In [19] the difficulty of the SE insertion in first order was avoided by computing the associated self-energy response as the imaginary part of the corresponding polarization propagator with OPE $\mathrm{SE}$ insertions on the particle and hole lines. A finite result was thus obtained in first order (one pionic line) without resorting to the HF approach. The question then arises whether it is possible to obtain the same result for the self-energy response function starting with finite well-defined matrix elements of the current operator.

In this paper we answer this question by constructing a renormalized self-energy current corresponding to OPE. This current acts over free Dirac spinors and leads to the same response functions as those obtained by taking the imaginary part of the polarization propagator computed to first order. It should be clear that in this work the concept of renormalization has a many-body significance, namely it amounts to a relativistic HF approximation and ignores (see [19]) the additional vacuum renormalization due to the change of the negative-energy sea induced by the nuclear medium [30].

The new current is obtained by renormalizing spinors and energies and by expanding the resulting in-medium one-body current to first order in the square of the pion-nucleon coupling constant, to be consistent with the requirement of dealing with diagrams having only one pionic line. The renormalized quantities should then be obtained in the general case by solving a set of self-consistent relativistic HF equations numerically. However, one of the goals of this paper is to show that to first order the solutions and the corresponding corrections to the bare single-nucleon current operator can be expressed analytically in terms of a simple electromagnetic operator. This operator accounts for two main effects induced by the interaction of the nucleon with the medium: the first is the enhancement of the lower components of the Dirac spinors; the second is a global renormalization 
of the spinors in the nuclear medium. These effects are genuine relativistic corrections that are absent in a non-relativistic framework [24]. Actually a third renormalization effect also arises, related to the in-medium modification of the energy-momentum relation for a nucleon, which is here treated in first order of the square of the pion-nucleon coupling constant (in other approaches this effect is embedded in a constant effective mass [29]).

Using the renormalized SE current operator together with the MEC and the vertex exchange operator we prove the full gauge invariance of the current if account is taken of the change in energy arising from the HF renormalization to first order. The results for the inclusive response functions we obtain with this current agree completely with the ones of [19], where the polarization propagator technique was used.

The present review is organized as follows: in Section 2 we focus on parity-conserving electron scattering from nuclei. We begin in Section 2.1 with some general formalism and then in Section 2.2 discuss the pion exchange and correlation currents. There we revisit the full set of $1 \mathrm{p}-1 \mathrm{~h}$ current operators with OPE line which contribute to the electro-excitation process, paying special attention to the SE contribution. We show the necessity of re-defining the otherwise infinite self-energy diagrams. In Section 2.3 we develop the HF renormalization scheme as a vehicle to addressing this problem, going on in Section 2.4 to expand the renormalized spinors and energies to first order in the pion coupling constant squared obtaining a new self-energy current. Then in Section 2.5 we prove the gauge invariance of the theory. To conclude this section we go on to discuss the hadronic tensor and electromagnetic response functions (Section 2.6) and present some typical results (Section 2.7). In Section 3 we briefly discuss parity-violating electron scattering to place it in context with the above studies. In Section 4 we make contact with non-relativistic expansions schemes, both for the pion-exchange currents (Section 4.1) and for the pionic correlations (Section 4.2). In Section 5 we summarize our results and draw our conclusions and end with a series of appendices where more technical aspects of the formalism are compiled.

\section{Parity-conserving electron scattering}

\subsection{General formalism}

The general formalism involved in the description of $\left(e, e^{\prime}\right)$ processes for quasielastic kinematics has been derived and discussed at length in several papers (see for instance [34-41]). Here we summarize only those aspects that are of special relevance to the analysis that follows. We limit our attention to the plane wave born approximation (PWBA), i.e., the electron is described as a plane wave and interacts with the nuclear target via the exchange of a virtual photon. The laboratory system variables involved in the process are $K^{\mu}=(\varepsilon, \mathbf{k})$ and $K^{\prime \mu}=\left(\varepsilon^{\prime}, \mathbf{k}^{\prime}\right)$, the initial and scattered electron four-momenta, and $P_{i}^{\mu}=\left(E_{i}, \mathbf{p}_{i}\right)=\left(M_{i}, \mathbf{0}\right)$ and $P_{f}^{\mu}=\left(E_{f}, \mathbf{p}_{f}\right)$, the initial and final hadronic four-momenta, respectively. The four-momentum transferred by the virtual photon is $Q^{\mu}=\left(K-K^{\prime}\right)^{\mu}=$ $\left(P_{f}-P_{i}\right)^{\mu}=(\omega, \mathbf{q})$; for electron scattering the momentum transfer is spacelike, $Q^{2}=\omega^{2}-q^{2}<0$, with $q=|\mathbf{q}|$. The $S$-matrix element in PWBA can then be written as

$$
S_{f i}=-2 \pi \mathrm{i} \delta\left(E_{f}-E_{i}-\omega\right) \frac{e^{2}}{Q^{2}}\left\langle\mathbf{k}^{\prime}, s^{\prime}\left|j_{\mathrm{e} \mu}(0)\right| \mathbf{k}, s\right\rangle\left\langle f\left|\hat{J}^{\mu}(Q)\right| i\right\rangle,
$$


where

$$
\left\langle\mathbf{k}^{\prime}, s^{\prime}\left|j_{\mathrm{e} \mu}(0)\right| \mathbf{k}, s\right\rangle=\left(\frac{m_{\mathrm{e}}}{V \varepsilon^{\prime}} \frac{m_{\mathrm{e}}}{V \varepsilon}\right)^{1 / 2} \bar{u}_{s^{\prime}}\left(\mathbf{k}^{\prime}\right) \gamma_{\mu} u_{s}(\mathbf{k})
$$

is the electron current matrix element and $\hat{J}^{\mu}(Q)$ is the Fourier transform of the nuclear electromagnetic current operator.

We assume Lorentz invariance, parity conservation and work in the extreme relativistic limit (ERL), in which the electron energy $\varepsilon \gg m_{\mathrm{e}}$. Under these conditions the unpolarized, inclusive $\left(e, e^{\prime}\right)$ cross section reads

$$
\frac{\mathrm{d} \sigma}{\mathrm{d} \Omega_{\mathrm{e}}^{\prime} \mathrm{d} \omega}=\frac{2 \alpha^{2}}{Q^{4}}\left(\frac{\varepsilon^{\prime}}{\varepsilon}\right) \eta_{\mu \nu} W^{\mu v}=\sigma_{\mathrm{M}}\left[v_{\mathrm{L}} R^{\mathrm{L}}(q, \omega)+v_{\mathrm{T}} R^{\mathrm{T}}(q, \omega)\right] .
$$

Here $\alpha$ is the fine structure constant and $\Omega_{\mathrm{e}}^{\prime}$ the scattered electron solid angle. The term $\sigma_{\mathrm{M}}$ represents the Mott cross section which in the ERL reduces to

$$
\sigma_{\mathrm{M}}=\left(\frac{\alpha \cos \theta_{\mathrm{e}} / 2}{2 \varepsilon \sin ^{2} \theta_{\mathrm{e}} / 2}\right)^{2}
$$

where $\theta_{\mathrm{e}}$ is the electron scattering angle, and $\eta_{\mu v}$ and $W^{\mu \nu}$ are the leptonic and hadronic tensor, respectively. Within PWBA the leptonic tensor simply reads

$$
\eta_{\mu \nu}=K_{\mu} K_{v}^{\prime}+K_{\mu}^{\prime} K_{v}+\frac{Q^{2}}{2} g_{\mu v} .
$$

The kinematic factors $v_{\mathrm{L}}$ and $v_{\mathrm{T}}$ are evaluated from the leptonic tensor using standard techniques (see, for example, [34])

$$
\begin{aligned}
& v_{\mathrm{L}}=\left(\frac{Q^{2}}{q^{2}}\right)^{2}, \\
& v_{\mathrm{T}}=-\frac{1}{2}\left(\frac{Q^{2}}{q^{2}}\right)+\tan ^{2} \frac{\theta_{\mathrm{e}}}{2},
\end{aligned}
$$

whereas the longitudinal and transverse (with respect to the momentum transfer $\mathbf{q}$ ) response functions $R^{\mathrm{L}}$ and $R^{\mathrm{T}}$ are constructed directly as components of the hadronic tensor $W^{\mu v}$ according to

$$
\begin{aligned}
& R^{\mathrm{L}}(q, \omega)=\left(\frac{q^{2}}{Q^{2}}\right)^{2}\left[W^{00}-\frac{\omega}{q}\left(W^{03}+W^{30}\right)+\frac{\omega^{2}}{q^{2}} W^{33}\right], \\
& R^{\mathrm{T}}(q, \omega)=W^{11}+W^{22}
\end{aligned}
$$

where we use a coordinate system with the $z$-axis in the direction of the vector q. Note that if gauge invariance is fulfilled, implying that $W^{03}=W^{30}=(\omega / q) W^{00}$ and $W^{33}=(\omega / q)^{2} W^{00}$, then $R^{\mathrm{L}}$ is simply the time component of the hadronic tensor, namely $W^{00}$. Hence $R^{\mathrm{L}}$ is determined by the charge distribution, whereas $R^{\mathrm{T}}$ reflects the current distribution of the nuclear target.

The hadronic tensor and consequently the response functions derived from it embody the entire dependence on the nuclear structure, specifically on the charge and current distributions in nuclei, and 
accordingly these provide the prime focus in analyses of electron scattering. There are various options on how to proceed in performing such analyses (see, for example, [42]), depending on the specific problem under consideration and on the approximations to be made. In what follows we recall two common expressions for the hadronic tensor $W^{\mu v}$ and comment briefly on their applications.

First, the hadronic tensor can be defined according to

$$
W^{\mu v}=\overline{\sum_{i}} \sum_{f}\left\langle f\left|\hat{J}^{\mu}(Q)\right| i\right\rangle^{*}\left\langle f\left|\hat{J}^{v}(Q)\right| i\right\rangle \delta\left(E_{i}+\omega-E_{f}\right),
$$

where $\hat{J}^{\mu}(Q)$ represents the nuclear many-body current operator, the nuclear states $|i\rangle$ and $|f\rangle$ are exact eigenstates of the nuclear Hamiltonian with definite four-momenta and the sum with a bar means average over initial states. This form is very general and includes all possible final states that can be reached through the action of the current operator $\hat{J}^{\mu}(Q)$ on the exact ground state. In our perturbative approach we shall use eigenstates of the free Hamiltonian $H_{0}$ (which describes the free relativistic Fermi gas) and include correlations among nucleons in the current mediated by the exchange of pions. This current of course allows one to reach both the $\mathrm{p}-\mathrm{h}$ and the $2 \mathrm{p}-2 \mathrm{~h}$ sectors in the Hilbert space of $H_{0}$. In the present work, however, we shall restrict our attention to the former.

A different option for evaluating the nuclear responses exploits the polarization propagator $\Pi^{\mu v}$ (also referred to as the current-current correlation function). The latter can be expressed in terms of the full propagator, $\hat{G}$, of the nuclear many-body system, since closure can be used to carry out the sum over the final states in Eq. (10). Then one has for the hadronic tensor [43]

$$
W^{\mu v}=-\frac{1}{\pi} \operatorname{Im} \Pi^{\mu v}(q, q ; \omega)=-\frac{1}{\pi} \operatorname{Im} \overline{\sum_{i}}\left\langle i\left|\hat{J}^{\dagger \mu}(Q) \hat{G}\left(\omega+E_{i}\right) \hat{J}^{v}(Q)\right| i\right\rangle .
$$

A possible advantage of this approach relates to the existence of a well-defined set of rules (the relativistic Feynman diagrams) which allows one to compute $\Pi^{\mu v}$ perturbatively [43].

Obviously, the two procedures are equivalent and hence the observables calculated using the expressions for the hadronic tensor given by Eq. (10) or (11) should be the same. However, notice that Eq. (10) is less suitable for dealing with situations where the nuclear current matrix element $\left\langle f\left|\hat{J}^{\mu}\right| i\right\rangle$ is divergent. In this case one proceeds either by computing directly the responses via the polarization propagator or by first renormalizing the current matrix element and then by using Eq. (10).

Finally, we remark that gauge invariance must be fulfilled both at the level of the nuclear current matrix elements and at the level of the hadronic tensor and/or the polarization propagator. A consequence is that the electromagnetic continuity equation should be satisfied. In other words, in momentum space all of the expressions $Q_{\mu}\left\langle f\left|\hat{J}^{\mu}(Q)\right| i\right\rangle, Q_{\mu} W^{\mu v}$ and $Q_{\mu} \Pi^{\mu v}$ should vanish.

\subsection{Pion-exchange and correlation currents}

Working within the framework of the RFG model, i.e., for nucleons moving freely inside the nucleus with relativistic kinematics, in this section we present a detailed study of the electromagnetic currents accounting for the effects introduced by pions in first-order perturbation theory (one-pion exchange). 


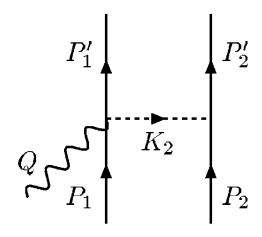

(a)

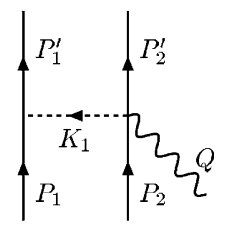

(b)

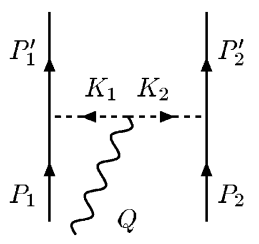

(c)

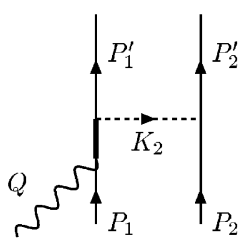

(d)

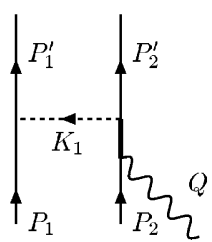

(e)

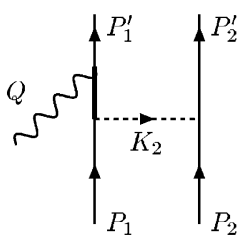

(f)

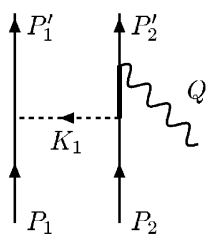

(g)

Fig. 1. Feynman diagrams contributing to the two-body current with OPE. The wide line in the correlation diagrams (d) $-(\mathrm{g})$ means a fully relativistic Dirac propagator for the nucleon.

\subsubsection{Feynman diagrams and two-body currents}

The linked, two-body Feynman diagrams that contribute to electron scattering with OPE are shown in Fig. 1. The first three correspond to the usual MEC: diagrams (a), (b) refer to the contact or seagull current, diagram (c) to the pion-in-flight current. The four diagrams (d) $-(\mathrm{g})$ represent the so-called correlation current and are usually not treated as genuine MEC, but as correlation corrections to the nuclear wave function. However, again we note that our approach puts all correlation effects in the current operator and uses an uncorrelated wave function for the initial and final nuclear states.

In this work we use Bjorken and Drell conventions [31] and pseudovector $\pi N N$ coupling (the effect of a pseudoscalar coupling will be commented on later), namely

$$
\mathscr{H}_{\pi N N}=\frac{f}{m_{\pi}} \bar{\psi} \gamma_{5} \gamma^{\mu}\left(\partial_{\mu} \phi_{a}\right) \tau_{a} \psi,
$$

where $\psi$ is the nucleon field, $\phi_{a}$ is the isovector pion field, $f$ represents the $\pi N N$ coupling constant and $m_{\pi}$ is the pion mass. The electromagnetic currents corresponding to diagrams (a) $-(\mathrm{g})$ are obtained by computing the $S$-matrix element

$$
S_{f i}=S_{f i}\left(P_{1}^{\prime}, P_{2}^{\prime}, P_{1}, P_{2}\right)-S_{f i}\left(P_{1}^{\prime}, P_{2}^{\prime}, P_{2}, P_{1}\right)
$$

for the absorption of a virtual photon by a system of two nucleons, namely for the process

$$
\gamma+N_{1}+N_{2} \rightarrow N_{1}^{\prime}+N_{2}^{\prime},
$$

with $P_{1}, P_{2}\left(P_{1}^{\prime}, P_{2}^{\prime}\right)$ being the initial (final) four-momenta of the two nucleons involved (see Fig. 1). The electromagnetic current is then defined according to

$$
S_{f i}\left(P_{1}^{\prime}, P_{2}^{\prime}, P_{1}, P_{2}\right)=-\mathrm{i} e A_{\mu}(Q) 2 \pi \delta\left(E_{1}^{\prime}+E_{2}^{\prime}-E_{1}-E_{2}-\omega\right)\left\langle P_{1}^{\prime} P_{2}^{\prime}\left|\hat{j}^{\mu}(Q)\right| P_{1} P_{2}\right\rangle,
$$


where $A_{\mu}(Q)$ is related to the matrix element of the electromagnetic field between the incident photon with momentum $Q$ and the vacuum state, namely

$$
\left\langle 0\left|A_{\mu}(X)\right| \gamma(Q)\right\rangle=A_{\mu}(Q) \mathrm{e}^{-\mathrm{i} Q \cdot X} .
$$

Finally, the on-shell matrix element of the two-body current can be written in terms of a function $j^{\mu}\left(\mathbf{p}_{1}^{\prime}, \mathbf{p}_{2}^{\prime}, \mathbf{p}_{1}, \mathbf{p}_{2}\right)$ as follows:

$$
\begin{aligned}
& \left\langle P_{1}^{\prime} P_{2}^{\prime}\left|\hat{j}^{\mu}(Q)\right| P_{1} P_{2}\right\rangle \\
& \quad=(2 \pi)^{3} \delta^{3}\left(\mathbf{p}_{1}^{\prime}+\mathbf{p}_{2}^{\prime}-\mathbf{q}-\mathbf{p}_{1}-\mathbf{p}_{2}\right) \frac{m^{2}}{V^{2}\left(E_{\mathbf{p}_{1}} E_{\mathbf{p}_{2}} E_{\mathbf{p}_{1}^{\prime}} E_{\mathbf{p}_{2}^{\prime}}\right)^{1 / 2}} j^{\mu}\left(\mathbf{p}_{1}^{\prime}, \mathbf{p}_{2}^{\prime}, \mathbf{p}_{1}, \mathbf{p}_{2}\right),
\end{aligned}
$$

where $m$ is the nucleon mass, $V$ is the volume enclosing the system and $E_{\mathbf{p}}=\sqrt{\mathbf{p}^{2}+m^{2}}$ the on-shell energy of a nucleon with momentum $\mathbf{p}$. The four-momenta - indicated by capital letters - are defined in Fig. 1.

The general relativistic expressions for the seagull (diagrams $(a, b)$ ), pion-in-flight (c) and correlation $(\mathrm{d}-\mathrm{g})$ current matrix elements are (isospin summations are understood)

- Seagull or contact:

$$
j_{\mathrm{s}}^{\mu}\left(\mathbf{p}_{1}^{\prime}, \mathbf{p}_{2}^{\prime}, \mathbf{p}_{1}, \mathbf{p}_{2}\right)=\frac{f^{2}}{m_{\pi}^{2}} \mathrm{i} \epsilon_{3 a b} \bar{u}\left(\mathbf{p}_{1}^{\prime}\right) \tau_{a} \gamma_{5} K_{1} u\left(\mathbf{p}_{1}\right) \frac{F_{1}^{\mathrm{V}}}{K_{1}^{2}-m_{\pi}^{2}} \bar{u}\left(\mathbf{p}_{2}^{\prime}\right) \tau_{b} \gamma_{5} \gamma^{\mu} u\left(\mathbf{p}_{2}\right)+(1 \leftrightarrow 2) .
$$

- Pion-in-flight:

$$
j_{\mathrm{p}}^{\mu}\left(\mathbf{p}_{1}^{\prime}, \mathbf{p}_{2}^{\prime}, \mathbf{p}_{1}, \mathbf{p}_{2}\right)=\frac{f^{2}}{m_{\pi}^{2}} \mathrm{i} \epsilon_{3 a b} \frac{F_{\pi}\left(K_{1}-K_{2}\right)^{\mu}}{\left(K_{1}^{2}-m_{\pi}^{2}\right)\left(K_{2}^{2}-m_{\pi}^{2}\right)} \bar{u}\left(\mathbf{p}_{1}^{\prime}\right) \tau_{a} \gamma_{5} K_{1} u\left(\mathbf{p}_{1}\right) \bar{u}\left(\mathbf{p}_{2}^{\prime}\right) \tau_{b} \gamma_{5} K_{2} u\left(\mathbf{p}_{2}\right) .
$$

- Correlation:

$$
\begin{aligned}
j_{\text {cor }}^{\mu}\left(\mathbf{p}_{1}^{\prime}, \mathbf{p}_{2}^{\prime}, \mathbf{p}_{1}, \mathbf{p}_{2}\right)= & \frac{f^{2}}{m_{\pi}^{2}} \bar{u}\left(\mathbf{p}_{1}^{\prime}\right) \tau_{a} \gamma_{5} K_{1} u\left(\mathbf{p}_{1}\right) \frac{1}{K_{1}^{2}-m_{\pi}^{2}} \bar{u}\left(\mathbf{p}_{2}^{\prime}\right)\left[\tau_{a} \gamma_{5} K_{1} S_{\mathrm{F}}\left(P_{2}+Q\right) \Gamma^{\mu}(Q)\right. \\
& \left.+\Gamma^{\mu}(Q) S_{\mathrm{F}}\left(P_{2}^{\prime}-Q\right) \tau_{a} \gamma_{5} K_{1}\right] u\left(\mathbf{p}_{2}\right)+(1 \leftrightarrow 2) .
\end{aligned}
$$

In the above, $K_{1}, K_{2}$ are the four-momenta given to the nucleons 1,2 by the exchanged pion, and they are defined in Fig. 1, while $F_{1}^{\mathrm{V}}$ and $F_{\pi}$ are the electromagnetic isovector nucleon and pion form factors, respectively. Furthermore, $S_{\mathrm{F}}(P)$ is the nucleon propagator and $\Gamma^{\mu}(Q)$ the electromagnetic nucleon vertex, i.e.,

$$
\begin{aligned}
& S_{\mathrm{F}}(P)=\frac{P+m}{P^{2}-m^{2}}, \\
& \Gamma^{\mu}(Q)=F_{1} \gamma^{\mu}+\frac{\mathrm{i}}{2 m} F_{2} \sigma^{\mu v} Q_{v},
\end{aligned}
$$

$F_{1}$ and $F_{2}$ being the Dirac and Pauli form factors: for these we use the Galster parameterization [44]. Finally, the spinors (for brevity we denote $u\left(\mathbf{p}, s_{p}\right)$ by $u(\mathbf{p})$ ) are normalized according to the Bjorken and Drell convention [31] and the isospinors are not explicitly indicated. 
The seagull and pion-in-flight currents shown above coincide with the expressions given by Van Orden and Donnelly [45] if account is taken for the different conventions used for the gamma matrix $\gamma_{5}$ and for the metric. Concerning the correlation current note that, at variance with [45], it embodies both the positive and negative energy components of the nucleon propagator.

A crucial point to be stressed is that the sum of the relativistic seagull, pion-in-flight and correlation currents satisfy current conservation, i.e., $Q_{\mu} J^{\mu}=0$, provided some assumptions are made for the form factors involved in the various currents. This is proven in Appendix A (see also [19]) where we show that when the seagull and pion-in-flight currents are multiplied by the same electromagnetic form factor $F_{1}^{\mathrm{V}}$, gauge invariance is fulfilled, i.e.,

$$
Q_{\mu}\left(j_{\mathrm{s}}^{\mu}+j_{\mathrm{p}}^{\mu}+j_{\mathrm{cor}}^{\mu}\right)=0,
$$

where the two-body currents in Eq. (23) are defined in Eqs. (18)-(20).

It is also possible [46] to use different phenomenological electromagnetic form factors for the nucleon and pion-even introducing phenomenological form factors at the strong pion-nucleon vertices-without violating current conservation, by appropriate modification in the currents through the generalized Ward-Takahashi identity [47,48].

\subsubsection{Particle-hole matrix elements}

In this report we deal with the case of one-particle emission induced by the two-body currents introduced above. The matrix element of a two-body operator between the Fermi gas ground state and a $1 \mathrm{p}-1 \mathrm{~h}$ excited state reads

$$
\begin{aligned}
\left\langle p h^{-1}\left|\hat{j}^{\mu}(Q)\right| F\right\rangle & \equiv(2 \pi)^{3} \delta^{3}(\mathbf{q}+\mathbf{h}-\mathbf{p}) \frac{m}{V \sqrt{E_{\mathbf{p}} E_{\mathbf{h}}}} j^{\mu}(\mathbf{p}, \mathbf{h}) \\
& =\sum_{k<F}\left[\left\langle p k\left|\hat{j}^{\mu}(Q)\right| h k\right\rangle-\left\langle p k\left|\hat{j}^{\mu}(Q)\right| k h\right\rangle\right],
\end{aligned}
$$

where the summation runs over all occupied levels in the ground state, and thus includes a sum over spin $\left(s_{k}\right)$ and isospin $\left(t_{k}\right)$ and an integral over the momentum $\mathbf{k}$.

The first and second terms in Eq. (24) represent the direct and exchange contribution to the matrix element, respectively. It can be easily verified (see, e.g., [19,20]) that in spin-isospin saturated systems, the direct term vanishes for the MEC and pionic correlation currents upon summation over the occupied states. Hence only the exchange term contributes to the $\mathrm{p}-\mathrm{h}$ matrix elements. The associated many-body Feynman diagrams are displayed in Fig. 2. Diagrams (a,b) and (c) correspond to the seagull (or contact) and pion-in-flight contributions, respectively. Diagrams ( $\mathrm{d}-\mathrm{g}$ ) represent instead the correlation contributions. Here we distinguish the exchange of a pion between a particle and a hole line $(\mathrm{d}, \mathrm{e})$, giving rise to the so-called vertex correlation (VC), and the self-energy insertions on the nucleonic lines (f,g). After carrying out explicitly the sums over the internal spin, $s_{k}$, and isospin, $t_{k}$, the fully relativistic expressions for the MEC (seagull and pion-in-flight) and correlation (vertex correlations and self-energy) currents turn out to be

- Seagull:

$$
j_{\mathrm{s}}^{\mu}(\mathbf{p}, \mathbf{h})=-\frac{f^{2}}{V m_{\pi}^{2}} F_{1}^{\mathrm{V}} \mathrm{i} \varepsilon_{3 a b} \sum_{\mathbf{k} \leqslant k_{\mathrm{F}}} \frac{m}{E_{\mathbf{k}}} \bar{u}(\mathbf{p}) \tau_{a} \tau_{b}\left\{\frac{(K-m) \gamma^{\mu}}{(P-K)^{2}-m_{\pi}^{2}}+\frac{\gamma^{\mu}(K-m)}{(K-H)^{2}-m_{\pi}^{2}}\right\} u(\mathbf{h}) .
$$




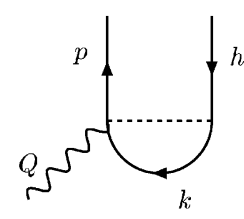

(a)

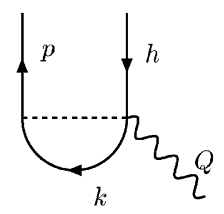

(b)

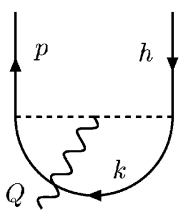

(c)

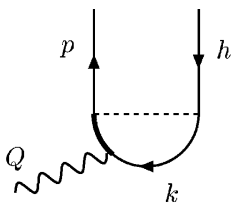

(d)

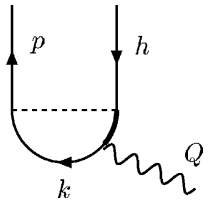

(e)

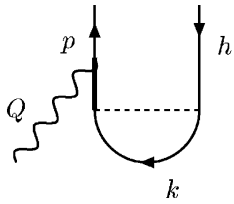

(f)

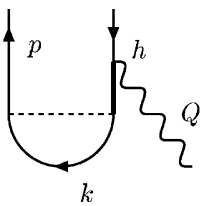

$(\mathrm{g})$

Fig. 2. Many-body Feynman diagrams contributing to the one-body current with OPE. The thick line in the correlation diagrams (d) $-(\mathrm{g})$ corresponds to a fully relativistic Dirac propagator for the nucleon. Diagrams (d) and (e) represent the vertex current, while diagrams ( $f$ and $g$ ) represent the self-energy current of the hole and of the particle, respectively.

- Pion-in-flight:

$$
\begin{aligned}
& j_{\mathrm{p}}^{\mu}(\mathbf{p}, \mathbf{h}) \\
& \quad=2 m \frac{f^{2}}{V m_{\pi}^{2}} F_{1}^{\mathrm{V}} \mathrm{i} \varepsilon_{3 a b} \sum_{\mathbf{k} \leqslant k_{\mathrm{F}}} \frac{m}{E_{\mathbf{k}}} \frac{(Q+2 H-2 K)^{\mu}}{\left[(P-K)^{2}-m_{\pi}^{2}\right]\left[(K-H)^{2}-m_{\pi}^{2}\right]} \bar{u}(\mathbf{p}) \tau_{a} \tau_{b}(K-m) u(\mathbf{h}) .
\end{aligned}
$$

- Vertex correlations:

$$
\begin{aligned}
j_{\mathrm{VC}}^{\mu}(\mathbf{p}, \mathbf{h}) & \\
= & \frac{f^{2}}{V m_{\pi}^{2}} \sum_{\mathbf{k} \leqslant k_{\mathrm{F}}} \frac{1}{2 E_{\mathbf{k}}} \bar{u}(\mathbf{p})\left\{\frac{K-H}{(K-H)^{2}-m_{\pi}^{2}} \gamma_{5} S_{\mathrm{F}}(K+Q) \tau_{a} \Gamma^{\mu}(Q) \tau_{a} \gamma_{5}(K-m)(K-H)\right. \\
& \left.+(P-K)(K-m) \gamma_{5} \tau_{a} \Gamma^{\mu}(Q) \tau_{a} S_{\mathrm{F}}(K-Q) \gamma_{5} \frac{P-\not K}{(P-K)^{2}-m_{\pi}^{2}}\right\} u(\mathbf{h}) \\
\equiv & \mathscr{F}^{\mu}+\mathscr{B}^{\mu} .
\end{aligned}
$$

- Self-energy:

$$
\begin{aligned}
j_{\mathrm{SE}}^{\mu}(\mathbf{p}, \mathbf{h})= & -\frac{3 f^{2}}{V m_{\pi}^{2}} \sum_{\mathbf{k} \leqslant k_{\mathrm{F}}} \frac{1}{2 E_{\mathbf{k}}} \bar{u}(\mathbf{p})\left\{\frac{P-K}{(P-K)^{2}-m_{\pi}^{2}}(K-m)(P-\not) S_{\mathrm{F}}(P) \Gamma^{\mu}(Q)\right. \\
& \left.+\Gamma^{\mu}(Q) S_{\mathrm{F}}(H)(K-H)(K-m) \frac{K-H}{(K-H)^{2}-m_{\pi}^{2}}\right\} u(\mathbf{h}) \\
\equiv & \mathscr{H}_{p}^{\mu}+\mathscr{H}_{h}^{\mu} .
\end{aligned}
$$

The effects of the medium are included through the summation in Eqs. (25)-(28) over the intermediate momentum $\mathbf{k}$ up to the Fermi momentum. 
In the thermodynamic limit the sum $(1 / V) \sum_{\mathbf{k} \leqslant k_{\mathrm{F}}}$ becomes an integral over the momentum $\int \mathrm{d}^{3} k /(2 \pi)^{3}$ in the range $0 \leqslant k \leqslant k_{\mathrm{F}}, k_{\mathrm{F}}$ being the Fermi momentum, and over the angular variables $\theta_{k}, \phi_{k}$. Note that, although the global factor $m / V \sqrt{E_{\mathbf{p}} E_{\mathbf{h}}}$ has been extracted from the current in Eq. (24), the factor $m / V E_{\mathbf{k}}$, associated with the internal line, has to be retained inside the sum. Note also that, in order to fulfill gauge invariance, we have assumed $F_{\pi}=F_{1}^{\mathrm{V}}$.

The vertex $\mathrm{p}-\mathrm{h}$ matrix element splits into two terms $\mathscr{F}^{\mu}$ and $\mathscr{B}^{\mu}$ representing the forward- and backward-going contributions, respectively (Fig. 2d and e). They are

$$
\begin{aligned}
& \mathscr{F}^{\mu}=-\frac{f^{2}}{V m_{\pi}^{2}} \sum_{\mathbf{k} \leqslant k_{\mathrm{F}}} \frac{m}{E_{\mathbf{k}}} \bar{u}(\mathbf{p}) \gamma_{5}(K-H) S_{\mathrm{F}}(K+Q) \tau_{a} \Gamma^{\mu}(Q) \tau_{a} \gamma_{5} \frac{(K-m)}{(K-H)^{2}-m_{\pi}^{2}} u(\mathbf{h}), \\
& \mathscr{B}^{\mu}=-\frac{f^{2}}{V m_{\pi}^{2}} \sum_{\mathbf{k} \leqslant k_{\mathrm{F}}} \frac{m}{E_{\mathbf{k}}} \bar{u}(\mathbf{p}) \frac{\not K-m}{(P-K)^{2}-m_{\pi}^{2}} \gamma_{5} \tau_{a} \Gamma^{\mu}(Q) \tau_{a} S_{\mathrm{F}}(K-Q) \gamma_{5}(P-K) u(\mathbf{h}) .
\end{aligned}
$$

Similarly, the self-energy $\mathrm{p}-\mathrm{h}$ matrix element splits into two terms, $\mathscr{H}_{p}^{\mu}$ and $\mathscr{H}_{h}^{\mu}$. The former corresponds to the diagram with the pion inserted in the particle line (Fig. $2 \mathrm{~g}$ ), whereas the latter describes the diagram with the pion inserted in the hole line (Fig. 2f). They are given by

$$
\begin{aligned}
& \mathscr{H}_{p}^{\mu}=-\frac{3 f^{2}}{V m_{\pi}^{2}} \sum_{\mathbf{k} \leqslant k_{\mathrm{F}}} \frac{m}{E_{\mathbf{k}}} \bar{u}(\mathbf{p})(K-m) \frac{P-K}{(P-K)^{2}-m_{\pi}^{2}} S_{\mathrm{F}}(P) \Gamma^{\mu}(Q) u(\mathbf{h}), \\
& \mathscr{H}_{h}^{\mu}=\frac{3 f^{2}}{V m_{\pi}^{2}} \sum_{\mathbf{k} \leqslant k_{\mathrm{F}}} \frac{m}{E_{\mathbf{k}}} \bar{u}(\mathbf{p}) \Gamma^{\mu}(Q) S_{\mathrm{F}}(H) \frac{(K-H)}{(K-H)^{2}-m_{\pi}^{2}}(K-m) u(\mathbf{h}) .
\end{aligned}
$$

Finally, splitting also the electromagnetic nucleon operator $\Gamma^{\mu}$ into its isoscalar and isovector parts, one obtains the isoscalar and isovector contributions to the self-energy and vertex $\mathrm{p}-\mathrm{h}$ matrix elements. The final results can be cast in the form

$$
\begin{aligned}
& \mathscr{F}^{\mu(\mathrm{S})}=-\frac{3 f^{2}}{V m_{\pi}^{2}} \sum_{\mathbf{k} \leqslant k_{\mathrm{F}}} \frac{m}{E_{\mathbf{k}}} \bar{u}(\mathbf{p}) \gamma_{5}(K-H /) S_{\mathrm{F}}(K+Q) \Gamma^{\mu(\mathrm{S})}(Q) \gamma_{5} \frac{(K-m)}{(K-H)^{2}-m_{\pi}^{2}} u(\mathbf{h}), \\
& \mathscr{B}^{\mu(\mathrm{S})}=-\frac{3 f^{2}}{V m_{\pi}^{2}} \sum_{\mathbf{k} \leqslant k_{\mathrm{F}}} \frac{m}{E_{\mathbf{k}}} \bar{u}(\mathbf{p}) \frac{\not K-m}{(P-K)^{2}-m_{\pi}^{2}} \gamma_{5} \Gamma^{\mu(\mathrm{S})}(Q) S_{\mathrm{F}}(K-Q) \gamma_{5}(\not P-K K) u(\mathbf{h})
\end{aligned}
$$

for the isoscalar and

$$
\begin{aligned}
\mathscr{F} \mu(\mathrm{V})= & -\frac{f^{2}}{V m_{\pi}^{2}} \sum_{\mathbf{k} \leqslant k_{\mathrm{F}}} \frac{m}{E_{\mathbf{k}}} \bar{u}(\mathbf{p}) \gamma_{5}(K-H) \\
& \times S_{\mathrm{F}}(K+Q) \Gamma^{\mu(\mathrm{V})}(Q)\left(\tau_{3}+\mathrm{i} \varepsilon_{3 a b} \tau_{a} \tau_{b}\right) \gamma_{5} \frac{(K-m)}{(K-H)^{2}-m_{\pi}^{2}} u(\mathbf{h}), \\
\mathscr{B}^{\mu(\mathrm{V})}= & -\frac{f^{2}}{V m_{\pi}^{2}} \sum_{\mathbf{k} \leqslant k \mathrm{~F}} \frac{m}{E_{\mathbf{k}}} \bar{u}(\mathbf{p}) \frac{K-m}{(P-K)^{2}-m_{\pi}^{2}} \gamma_{5} \Gamma^{\mu(\mathrm{V})}(Q)\left(\tau_{3}+\mathrm{i} \varepsilon_{3 a b} \tau_{a} \tau_{b}\right) \\
& \times S_{\mathrm{F}}(K-Q) \gamma_{5}(P P-K X) u(\mathbf{h})
\end{aligned}
$$


for the isovector vertex $\mathrm{p}-\mathrm{h}$ matrix elements and

$$
\begin{aligned}
& \mathscr{H}_{p}^{\mu(\mathrm{S}, \mathrm{V})} \\
& \quad=-\frac{3 f^{2}}{2 V m_{\pi}^{2}} \sum_{\mathbf{k} \leqslant k_{\mathrm{F}}} \frac{m}{E_{\mathbf{k}}} \bar{u}(\mathbf{p}) \frac{P-K}{(P-K)^{2}-m_{\pi}^{2}}(K-m)(P-K) S_{\mathrm{F}}(P) \Gamma^{\mu(\mathrm{S}, \mathrm{V})}(Q) u(\mathbf{h}), \\
& \mathscr{H}_{h}^{\mu(\mathrm{S}, \mathrm{V})} \\
& \quad=-\frac{3 f^{2}}{2 V m_{\pi}^{2}} \sum_{\mathbf{k} \leqslant k_{\mathrm{F}}} \frac{m}{E_{\mathbf{k}}} \bar{u}(\mathbf{p}) \Gamma^{\mu(\mathrm{S}, \mathrm{V})}(Q) S_{\mathrm{F}}(H)(K-H)(K-m) \frac{(K-H /)}{(K-H)^{2}-m_{\pi}^{2}} u(\mathbf{h})
\end{aligned}
$$

for the self-energy matrix elements. Interestingly, the isoscalar/isovector ratio is $=-3$ in the vertex matrix element, whereas in the self-energy case it is the unity. ${ }^{1}$ Note that the MEC (pion-in-flight and seagull) $\mathrm{p}-\mathrm{h}$ matrix elements are purely isovector, whereas the vertex and self-energy correlations get both isoscalar and isovector contributions.

The VC and SE $\mathrm{p}-\mathrm{h}$ matrix elements involve the nucleon propagator $S_{\mathrm{F}}(P)$ which in some situations may imply the occurrence of singularities. In the case of the vertex diagrams, the four-momenta appearing in the propagators are $K+Q$ and $K-Q$ for the forward- (Fig. 2d) and backward-going (Fig. 2e) contributions, respectively, and an integration over $\mathbf{k}$ should be done. For $q \geqslant 2 k_{\mathrm{F}}$ (no Pauli blocking) it can be proven (see [19]) that only the forward diagram contains a pole, i.e., a value of the inner momentum $\mathbf{k}$ exists such that the nucleon carrying a four-momentum $K+Q$ is on-shell. In this situation the forward vertex $\mathrm{p}-\mathrm{h}$ matrix element is evaluated by taking the principal value in the integral over $\cos \theta_{k}$. In the case of the backward-going diagram, the nucleon propagator $S_{\mathrm{F}}(K-Q)$ has no singularity for the kinematics in which we are interested.

The case of the self-energy diagrams is clearly different. Here the particle $(p)$ and hole $(h)$ are described in the Fermi gas by unperturbed plane waves, i.e., they are on-shell, and hence the propagators $S_{\mathrm{F}}(P)$ and $S_{\mathrm{F}}(H)$ diverge. The divergence of diagrams (f) and (g) is reminiscent of the well-known infinity occurring in standard perturbative quantum field theory, when self-energy insertions in the external legs are included in Feynman diagrams [32]. As is well known, there one should renormalize the theory by dressing the external legs, propagators and vertices. In the nuclear matter case we assume that the particle-physics effects are already accounted for by the physical masses and electromagnetic form factors. However, an additional nuclear physics renormalization, arising from the interaction of a nucleon with the nuclear medium, should be included at the OPE level to account for the self-energy diagram.

The self-energy current in Eq. (28) can be written in the following form:

$$
j_{\mathrm{SE}}^{\mu}(\mathbf{p}, \mathbf{h})=\bar{u}(\mathbf{p}) \Sigma(P) S_{\mathrm{F}}(P) \Gamma^{\mu}(Q) u(\mathbf{h})+\bar{u}(\mathbf{p}) \Gamma^{\mu}(Q) S_{\mathrm{F}}(H) \Sigma(H) u(\mathbf{h}),
$$

\footnotetext{
${ }^{1}$ The latter result stems from the relation $\tau_{3}+\mathrm{i} \varepsilon_{3 a b} \tau_{a} \tau_{b}=-\tau_{3}$; however, we prefer to leave the isospin structure of the isovector exchange as in Eqs. (35) and (36), since it makes more transparent the self-energy and exchange cancellation in the continuity equation, as shown in Appendix B.
} 


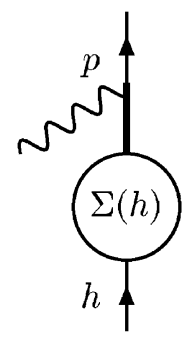

(a)

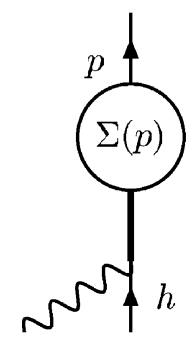

(b)

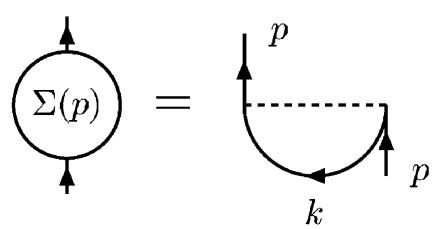

(c)

Fig. 3. Diagrammatic representation of the self-energy current for a hole (a) and a particle (b). The self-energy is defined to first order as the Fock insertion shown in (c) with OPE.

where $\Sigma(P)$ is the nucleon self-energy matrix that in first order reads

$$
\Sigma(P)=-\frac{f^{2}}{V m_{\pi}^{2}} \sum_{\mathbf{k} \leqslant k_{\mathrm{F}}} \sum_{s_{k}, t_{k}} \frac{m}{E_{\mathbf{k}}} \tau_{a} \gamma_{5}(P-K) \frac{u(\mathbf{k}) \bar{u}(\mathbf{k})}{(P-K)^{2}-m_{\pi}^{2}} \tau_{a} \gamma_{5}(P-\not K) .
$$

This is diagrammatically displayed in Fig. 3. The SE matrix, shown in Fig. 3c, corresponds to the Fock term of the mean-field potential (the Hartree or direct term is zero for pion exchange, since it involves a pion carrying zero momentum).

Performing the sum over the internal spin $\left(s_{k}\right)$ and isospin $\left(t_{k}\right)$ indices and using the commutation properties of the gamma matrices to eliminate $\gamma_{5}$, the self-energy can be cast in the form

$$
\Sigma(P)=-\frac{3 f^{2}}{m_{\pi}^{2}} \int \frac{\mathrm{d}^{3} k}{(2 \pi)^{3}} \theta\left(k_{\mathrm{F}}-k\right) \frac{1}{2 E_{\mathbf{k}}} \frac{(P-K)(K-m)(P-K)}{(P-K)^{2}-m_{\pi}^{2}},
$$

where the sum over $\mathbf{k}$ has been converted into an integral. Note that the self-energies $\Sigma(P)$ and $\Sigma(H)$ appearing in Eq. (39) are evaluated for free particles and holes, i.e., for $P^{\mu}$ and $H^{\mu}$ on-shell. Hence the self-energy contributions to the current are divergent, since so are the free propagators $S_{\mathrm{F}}(P)$ and $S_{\mathrm{F}}(H)$ in Eq. (39). Therefore they should not be computed using Eq. (39), but rather one should first renormalize the wave function and the propagator of the particles in the medium. This is achieved through the summation of the full series of diagrams with repeated self-energy insertions displayed in Fig. 4.

Now the energy of a particle in nuclear matter is modified by the interaction with the medium and, as well, through its energy-momentum relation. Thus the associated momentum is no longer 


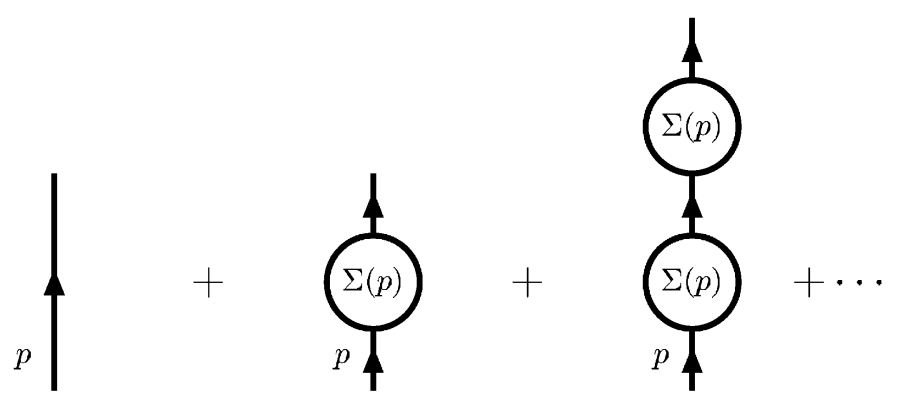

Fig. 4. Diagrammatic series for the nucleon propagator in the medium.

on-shell and therefore in the next section we shall evaluate the self-energy for off-shell particles. In the first iteration, corresponding to OPE, the particle $P^{\mu}$ is off-shell, but the intermediate interacting hole $K^{\mu}$ is still on-shell. ${ }^{2}$ In this case, with the help of Dirac spinology, one writes

$$
(P-K)(K-m)(P-K)=2\left(P \cdot K-m^{2}\right)(P+m)-\left(P^{2}-m^{2}\right)(K+m),
$$

which allows one to recast the self-energy in Eq. (41) for the off-shell momentum $P$ in the form

$$
\Sigma(P)=-\frac{3 f^{2}}{m_{\pi}^{2}} \int \frac{\mathrm{d}^{3} k}{(2 \pi)^{3}} \theta\left(k_{\mathrm{F}}-k\right) \frac{1}{2 E_{\mathbf{k}}} \frac{2\left(P \cdot K-m^{2}\right)(P+m)-\left(P^{2}-m^{2}\right)(K+m)}{(P-K)^{2}-m_{\pi}^{2}} .
$$

Note that the second term inside the integral vanishes for $P$ on-shell.

In general the self-energy of a nucleon in nuclear matter can be written in the form [49]

$$
\Sigma(P)=m A(P)+B(P) \gamma_{0} p^{0}-C(P) \gamma \cdot \mathbf{p} .
$$

In contrast to the quantum field-theory decomposition $\Sigma(P)=m A+B P$, owing to the non-invariance under a boost of the step function $\theta\left(k_{\mathrm{F}}-k\right)$ appearing in the self-energy, in nuclear matter $B(P) \neq$ $C(P)$. This in turn reflects the existence of a privileged system, namely the lab system where the Fermi gas has total momentum $\mathbf{p}_{\mathrm{FG}}=0$. Here it is natural to compute the self-energy. Under a boost, the Fermi gas ground state is no longer characterized by $k<k_{\mathrm{F}}$ and also the self-energy takes a different form.

In the case of the Fock self-energy in Eq. (43) the functions $A, B, C$ can be expressed in terms of the integrals (for $K^{\mu}$ on-shell)

$$
\begin{aligned}
& I(P) \equiv \int \frac{\mathrm{d}^{3} k}{(2 \pi)^{3}} \theta\left(k_{\mathrm{F}}-k\right) \frac{1}{2 E_{\mathbf{k}}} \frac{1}{(P-K)^{2}-m_{\pi}^{2}}, \\
& L^{\mu}(P) \equiv \int \frac{\mathrm{d}^{3} k}{(2 \pi)^{3}} \theta\left(k_{\mathrm{F}}-k\right) \frac{1}{2 E_{\mathbf{k}}} \frac{K^{\mu}}{(P-K)^{2}-m_{\pi}^{2}} .
\end{aligned}
$$

\footnotetext{
${ }^{2}$ Note that in deriving Eq. (41) we have assumed free spinors $u(\mathbf{k})$; hence Eq. (41) is only valid for $K^{\mu}$ on-shell. The off-shell case requires a redefinition of the spinors $u(\mathbf{k})$ according to an interacting Dirac equation, as is shown later.
} 
Indeed one gets ${ }^{3}$

$$
\begin{aligned}
& A(P)=-3 \frac{f^{2}}{m_{\pi}^{2}}\left\{2\left[P_{\mu} L^{\mu}(P)-m^{2} I(P)\right]-\left(P^{2}-m^{2}\right) I(P)\right\}, \\
& B(P)=-3 \frac{f^{2}}{m_{\pi}^{2}}\left\{2\left[P_{\mu} L^{\mu}(P)-m^{2} I(P)\right]-\left(P^{2}-m^{2}\right) \frac{L_{0}(P)}{p_{0}}\right\}, \\
& C(P)=-3 \frac{f^{2}}{m_{\pi}^{2}}\left\{2\left[P_{\mu} L^{\mu}(P)-m^{2} I(P)\right]-\left(P^{2}-m^{2}\right) \frac{L_{3}(P)}{p}\right\} .
\end{aligned}
$$

Note that $A=B=C$ for $P$ on-shell. In this case one simply has $\Sigma(P)_{\text {on-shell }}=A(P)(m+P)$.

\subsection{Hartree-Fock renormalization in nuclear matter}

In this section we discuss the renormalization of the nucleon propagator and spinors associated with the pionic self-energy in an HF scheme.

\subsubsection{Nucleon propagator}

The HF nucleon propagator in the nuclear medium is the solution of Dyson's equation

$$
S_{\mathrm{HF}}(P)=S_{0}(P)+S_{0}(P) \Sigma(P) S_{\mathrm{HF}}(P),
$$

where $\Sigma(P)$ is the HF proper self-energy and

$$
S_{0}(P)=\frac{\theta\left(p-k_{\mathrm{F}}\right)}{P+m+\mathrm{i} \epsilon}+\frac{\theta\left(k_{\mathrm{F}}-p\right)}{P+m-\mathrm{i} \epsilon p_{0}}
$$

is the free propagator in the medium. Eq. (50) results from summing up a series with an infinite number of self-energy insertions (see Fig. 4) for each of the two terms in (51), ${ }^{4}$ namely

$$
\begin{aligned}
& \frac{1}{P-m}+\frac{1}{P-m} \Sigma(P) \frac{1}{P-m}+\frac{1}{P-m} \Sigma(P) \frac{1}{P-m} \Sigma(P) \frac{1}{P-m}+\cdots \\
& =\frac{1}{P-m-\Sigma(P)} .
\end{aligned}
$$

Using the spin decomposition of the self-energy in Eq. (44), we can write

$$
P-m-\Sigma(P)=[1-B(P)] \gamma_{0} p_{0}-[1-C(P)] \gamma \cdot \mathbf{p}-[1+A(P)] m .
$$

\footnotetext{
${ }^{3} \mathbf{L}$ is parallel to $\mathbf{p}$ since, choosing $\mathbf{p}$ along the $z$-axis, the azimuthal integration in Eq. (46) yields $L_{x}=L_{y}=0$.

${ }^{4}$ No interference term arises since $\theta\left(k-k_{\mathrm{F}}\right) \theta\left(k_{\mathrm{F}}-k\right)=0$.
} 
Now the new four-momentum $f^{\mu}=f^{\mu}(P)$, which is related to $P^{\mu}$ as

$$
\begin{aligned}
& f_{0}(P)=\frac{1-B(P)}{1-C(P)} p_{0}, \\
& \mathbf{f}(P)=\mathbf{p}
\end{aligned}
$$

and the functions

$$
\begin{aligned}
& \tilde{m}(P)=\frac{1+A(P)}{1-C(P)} m, \\
& z(P)=\frac{1}{1-C(P)},
\end{aligned}
$$

allow one to recast Eq. (52) in the form

$$
\frac{1}{P-m-\Sigma(P)}=\frac{z(P)}{\gamma_{0} f_{0}(P)-\boldsymbol{\gamma} \cdot \mathbf{p}-\tilde{m}(P)}=\frac{z(P)}{f(P)-\tilde{m}(P)} .
$$

For a nucleon with a fixed three-momentum p, the pole of the propagator in Eq. (58) in the variable $p_{0}$ defines the new energy of the nucleon in the medium. To find the latter we introduce

$$
\tilde{E}(P) \equiv E(\mathbf{p}, \tilde{m}(P))=\sqrt{\mathbf{p}^{2}+\tilde{m}(P)^{2}} .
$$

Then the propagator reads

$$
\frac{1}{P-m-\Sigma(P)}=\frac{z(P)}{f_{0}(P)-\tilde{E}(P)} \frac{f(P)+\tilde{m}(P)}{f_{0}(P)+\tilde{E}(P)}
$$

and its pole $p_{0}$ is found by solving the implicit equation

$$
f_{0}(P)=\tilde{E}(P)
$$

which, exploiting Eq. (54), can be recast as follows:

$$
p_{0}=\frac{1-C(P)}{1-B(P)} \sqrt{\mathbf{p}^{2}+\tilde{m}(P)^{2}} \equiv \frac{1-C\left(p_{0}, \mathbf{p}\right)}{1-B\left(p_{0}, \mathbf{p}\right)} \sqrt{\mathbf{p}^{2}+\tilde{m}\left(p_{0}, \mathbf{p}\right)^{2}} .
$$

The solution of Eq. (62) for fixed $\mathbf{p}$ defines the new dispersion relation $p_{0}=\epsilon(\mathbf{p})$ for interacting nuclear matter. Once the above equation has been solved, the field strength renormalization constant

$$
Z_{2}(\mathbf{p})=\left.\operatorname{Res} \frac{z(P)}{f_{0}(P)-\tilde{E}(P)}\right|_{p_{0}=\epsilon(\mathbf{p})},
$$

defined as the residue of the first factor on the right-hand side of Eq. (60) at $p_{0}=\epsilon(\mathbf{p})$, can be computed. Indeed using Eq. (57), $Z_{2}(\mathbf{p})$ is obtained by expanding the denominator around the pole $\epsilon(\mathbf{p})$, i.e.,

$$
[1-C(P)]\left[f_{0}(P)-\tilde{E}(P)\right]=Z_{2}(\mathbf{p})^{-1}\left[p_{0}-\epsilon(\mathbf{p})\right]+\cdots ;
$$


hence

$$
\begin{aligned}
Z_{2}(\mathbf{p})^{-1} & =\left.\frac{\partial}{\partial p_{0}}\right|_{p_{0}=\epsilon(\mathbf{p})}\left\{[1-C(P)]\left[f_{0}(P)-\tilde{E}(P)\right]\right\} \\
& =\left.\frac{\partial}{\partial p_{0}}\right|_{p_{0}=\epsilon(\mathbf{p})}\left\{[1-B(P)] p_{0}-[1-C(P)] \tilde{E}(P)\right\} .
\end{aligned}
$$

With the help of Eq. (59) the derivative can be easily evaluated, the result being

$$
Z_{2}(\mathbf{p})^{-1}=\left[1-B-\frac{\partial B}{\partial p_{0}} p_{0}-m \frac{\tilde{m}}{\tilde{E}} \frac{\partial A}{\partial p_{0}}+\frac{\mathbf{p}^{2}}{\tilde{E}} \frac{\partial C}{\partial p_{0}}\right]_{p_{0}=\epsilon(\mathbf{p})} .
$$

\subsubsection{Nucleon spinors}

The self-energy modifies not only the propagator and the energy-momentum relation of a nucleon, but, as well, the free Dirac spinors. In fact the spinors are now solutions of the Dirac equation in the nuclear medium [49], i.e.,

$$
[P-m-\Sigma(P)] \phi(\mathbf{p})=0
$$

which, again using the decomposition in Eq. (44), can be recast as

$$
\left[\gamma_{0} f_{0}(P)-\gamma \cdot \mathbf{p}-\tilde{m}(P)\right] \phi(\mathbf{p})=0,
$$

the functions $f_{0}(P)$ and $\tilde{m}(P)$ being defined in Eqs. (54) and (56), respectively. Eq. (68) has the same structure as the free Dirac equation; hence for the positive-energy eigenvalue one has

$$
f_{0}^{2}(P)=\mathbf{p}^{2}+\tilde{m}^{2}(P)
$$

which implicitly yields the energy $p_{0}=\epsilon(\mathbf{p})$ of the nucleon in the nuclear medium. This result was already obtained as the pole of the nucleon propagator. Then the corresponding positive-energy spinors ( $s$ being the spin index) read

$$
\phi_{s}(\mathbf{p})=\sqrt{Z_{2}(\mathbf{p})}\left(\frac{\tilde{E}(\mathbf{p})+\tilde{m}(\mathbf{p})}{2 \tilde{m}(\mathbf{p})}\right)^{1 / 2}\left[\frac{\boldsymbol{\sigma} \cdot \mathbf{p}}{\tilde{E}(\mathbf{p})+\tilde{m}(\mathbf{p})} \chi_{s}\right]=\sqrt{Z_{2}(\mathbf{p})} u_{s}(\mathbf{p}, \tilde{m}(\mathbf{p})),
$$

where the two functions of the three-momentum $\mathbf{p}$

$$
\tilde{m}(\mathbf{p}) \equiv \tilde{m}(\epsilon(\mathbf{p}), \mathbf{p})
$$

and

$$
\tilde{E}(\mathbf{p}) \equiv \tilde{E}(\epsilon(\mathbf{p}), \mathbf{p})=\sqrt{\mathbf{p}^{2}+\tilde{m}(\mathbf{p})^{2}},
$$

represent the nucleon effective mass and effective energy corresponding to $p_{0}=\epsilon(\mathbf{p})$. The field strength renormalization constant, $\sqrt{Z_{2}(\mathbf{p})}$, of the new spinors, defined in Eq. (66), is required by renormalization theory, since the propagator in Eq. (58) for $p_{0}$ close to the pole $\epsilon(\mathbf{p})$ reads, 


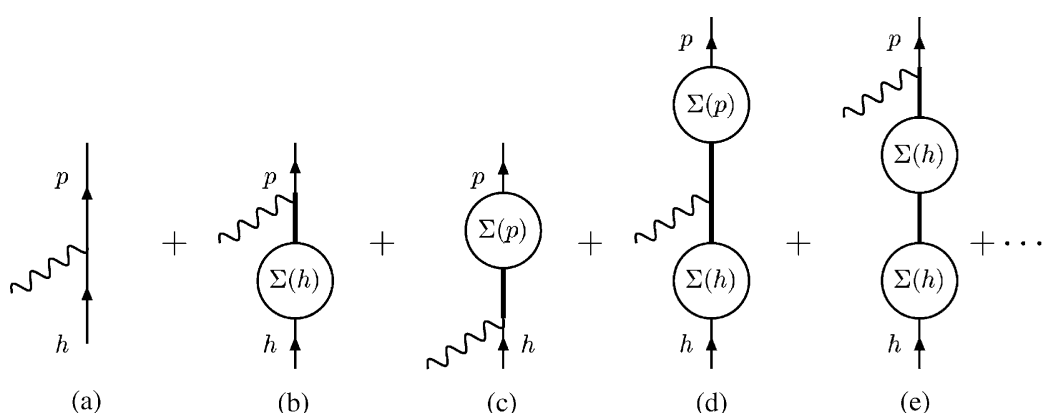

Fig. 5. Diagrammatic series for the one-body electromagnetic current with dressed external lines.

from Eq. (64),

$$
\frac{1}{P-m-\Sigma(P)} \sim \frac{Z_{2}(\mathbf{p})}{p_{0}-\epsilon(\mathbf{p})} \frac{f(\mathbf{p})+\tilde{m}(\mathbf{p})}{2 \tilde{E}(\mathbf{p})}=\frac{1}{p_{0}-\epsilon(\mathbf{p})} \frac{\tilde{m}(\mathbf{p})}{\tilde{E}(\mathbf{p})} \sum_{s} \phi_{s}(\mathbf{p}) \bar{\phi}_{s}(\mathbf{p}) .
$$

Once the new spinors have been computed, the self-energy can be evaluated by inserting $\phi(\mathbf{k})$ instead of $u(\mathbf{k})$ into Eq. (40). Then the Dirac equation should be solved again with the new self-energy and so on. This self-consistent procedure leads to the relativistic HF model which has to be dealt with numerically.

In this paper we do not attempt to solve the HF equations, since we are interested only in the OPE first-iteration correction to the single-nucleon current. Although the latter cannot be derived by directly applying the Feynman rules, it can still be identified with the self-energy diagrams of Fig. $2 \mathrm{f}$ and $\mathrm{g}$. Thus in the next section we shall compute the renormalized one-body current using the new spinors and energy-momentum relation and then expand it in powers of the square of the pion coupling constant $f^{2} / m_{\pi}^{2}$. As we shall see, the unperturbed one-body current is thus recovered as the leading-order term whereas the first-order term is searched for the self-energy contribution.

It is also important to remark that the use of the new 'renormalized' wave functions $\phi_{s}$ leads to a slightly modified global momentum distribution as shown in [50]. Note however that the number of particles is conserved without modifying the value of the Fermi momentum selected.

\subsection{Self-energy current to first order}

The particle-hole $(\mathrm{p}-\mathrm{h})$ current matrix element in the HF approximation reads

$$
j_{\mathrm{HF}}^{\mu}(\mathbf{p}, \mathbf{h})=\bar{\phi}(\mathbf{p}) \Gamma^{\mu}(Q) \phi(\mathbf{h}),
$$

where the spinors $\phi(\mathbf{p})$, the first iteration solution of the HF equation, are given by Eq. (70). Hence Eq. (74) represents the electromagnetic excitation of the $\mathrm{p}-\mathrm{h}$ pair with dressed external lines corresponding to the sum of the diagrams shown in Fig. 5.

In order to obtain a genuine OPE expression we expand Eq. (74) in powers of the square of the pion coupling constant $f^{2} / m_{\pi}^{2}$ and single out the first-order term, i.e., the one linear in $f^{2} / m_{\pi}^{2}$. We shall still refer to the current thus obtained, representing the OPE contribution, as the "self-energy" current and, importantly, we shall show that it yields a finite contribution, free from the divergence problem of the current in Eq. (39). 
To proceed we start by deriving the HF energy $\epsilon(\mathbf{p})$ to first order in $f^{2} / m_{\pi}^{2}$. For this purpose we note that the functions $A(P), B(P)$ and $C(P)$ defined in Eqs. (47)-(49) are of order $O\left(f^{2} / m_{\pi}^{2}\right)$. Hence the following expansion of the Dirac mass in Eq. (56) holds:

$$
\tilde{m}(P)=m \frac{1+A(P)}{1-C(P)}=m[1+A(P)+C(P)]+O\left(\frac{f^{4}}{m_{\pi}^{4}}\right) .
$$

Inserting this into Eq. (62) for the energy and expanding again to first order in $f^{2} / m_{\pi}^{2}$, we get

$$
\begin{aligned}
p_{0}=\epsilon(\mathbf{p}) & \simeq[1-C(P)+B(P)] \sqrt{\mathbf{p}^{2}+m^{2}+2 m^{2}[A(P)+C(P)]} \\
& =E_{\mathbf{p}}+\Delta E\left(p_{0}, \mathbf{p}\right),
\end{aligned}
$$

where $E_{\mathbf{p}}=\sqrt{\mathbf{p}^{2}+m^{2}}$ is the unperturbed free energy and

$$
\Delta E\left(p_{0}, \mathbf{p}\right) \equiv \frac{1}{E_{\mathbf{p}}}\left[m^{2} A(P)+E_{\mathbf{p}}^{2} B(P)-\mathbf{p}^{2} C(P)\right]+O\left(\frac{f^{4}}{m_{\pi}^{4}}\right)
$$

is the first-order correction to the energy. Next we can insert the above value of $p_{0}$ inside the argument of the functions $A, B, C$. Expanding the latter around the on-shell value $p_{0}=E_{\mathbf{p}}$ we get

$$
A(P)=A\left(p_{0}, \mathbf{p}\right)=A\left(E_{\mathbf{p}}+\Delta E, \mathbf{p}\right)=A\left(E_{\mathbf{p}}, \mathbf{p}\right)+O\left(\frac{f^{4}}{m_{\pi}^{4}}\right) \simeq A_{0}(\mathbf{p}),
$$

where $A_{0}(\mathbf{p}) \equiv A\left(E_{\mathbf{p}}, \mathbf{p}\right)$. Likewise to first order we obtain

$$
\begin{aligned}
& B(P) \simeq B\left(E_{\mathbf{p}}, \mathbf{p}\right) \equiv B_{0}(\mathbf{p}), \\
& C(P) \simeq C\left(E_{\mathbf{p}}, \mathbf{p}\right) \equiv C_{0}(\mathbf{p}) .
\end{aligned}
$$

Recalling that for $P$ on-shell the functions $A, B, C$ coincide, i.e., $A_{0}(\mathbf{p})=B_{0}(\mathbf{p})=C_{0}(\mathbf{p})$, we can insert these on-shell values into Eq. (77) and, neglecting terms of second order, i.e., $O\left(f^{4} / m_{\pi}^{4}\right)$, we finally arrive at the result

$$
p_{0}=\epsilon(\mathbf{p})=E_{\mathbf{p}}+\frac{1}{E_{\mathbf{p}}} B_{0}(\mathbf{p})\left(m^{2}+E_{\mathbf{p}}^{2}-\mathbf{p}^{2}\right)+O\left(\frac{f^{4}}{m_{\pi}^{4}}\right)=E_{\mathbf{p}}+\frac{1}{E_{\mathbf{p}}} 2 m^{2} B_{0}(\mathbf{p})+O\left(\frac{f^{4}}{m_{\pi}^{4}}\right) .
$$

The above expression can be recast in terms of the on-shell value of the self-energy

$$
\Sigma_{0}(\mathbf{p}) \equiv 2 m B_{0}(\mathbf{p}),
$$

which satisfies the relation

$$
\Sigma\left(E_{\mathbf{p}}, \mathbf{p}\right) u(\mathbf{p})=\Sigma_{0}(\mathbf{p}) u(\mathbf{p}),
$$

thus showing that the free spinors are eigenvectors of the on-shell self-energy matrix $\Sigma\left(E_{\mathbf{p}}, \mathbf{p}\right)$ corresponding to the eigenvalue $\Sigma_{0}(\mathbf{p})$. Hence to first order in $f^{2} / m_{\pi}^{2}$, the HF energy in Eq. (81) is found to read

$$
\epsilon(\mathbf{p}) \simeq E_{\mathbf{p}}+\frac{m}{E_{\mathbf{p}}} \Sigma_{0}(\mathbf{p})
$$


in terms of the on-shell self-energy eigenvalue $\Sigma_{0}(\mathbf{p})$. When compared with the non-relativistic HF energy (see Eq. (207)) it appears that, beyond the different expressions of the self-energy functions that hold in the relativistic and non-relativistic frameworks, an extra multiplicative factor $m / E_{\mathbf{p}}$ occurs in the relativistic case.

Once the HF energy $\epsilon(\mathbf{p})$ is known to first order in $f^{2} / m_{\pi}^{2}$, we expand as well the renormalized spinors, namely

$$
\sqrt{\frac{\tilde{m}(\mathbf{p})}{\tilde{E}(\mathbf{p})}} u(\mathbf{p}, \tilde{m}(\mathbf{p}))=\sqrt{\frac{\tilde{E}(\mathbf{p})+\tilde{m}(\mathbf{p})}{2 \tilde{E}(\mathbf{p})}}\left[\begin{array}{c}
\chi \\
\frac{\boldsymbol{\sigma} \cdot \mathbf{p}}{\tilde{E}(\mathbf{p})+\tilde{m}(\mathbf{p})} \chi
\end{array}\right] .
$$

Actually, for later use in the calculation of the hadronic tensor, it is convenient to expand the spinor multiplied by the factor $\sqrt{\tilde{m}(\mathbf{p}) / \tilde{E}(\mathbf{p})}$.

Thus we start by expanding the Dirac mass in Eq. (75) around the on-shell energy, obtaining

$$
\tilde{m}(\mathbf{p})=m\left[1+A_{0}(\mathbf{p})+C_{0}(\mathbf{p})\right]+O\left(f^{2} / m_{\pi}^{2}\right) \simeq m+\Sigma_{0}(\mathbf{p}),
$$

where use has been made of the on-shell self-energy in Eq. (82). Likewise, using the HF equation (Eq. (62)), the Dirac energy $\tilde{E}(\mathbf{p})$ defined in Eq. (72) is given by

$$
\begin{aligned}
\tilde{E}(\mathbf{p}) & =\frac{1-B}{1-C} \epsilon(\mathbf{p}) \simeq\left[1-B_{0}(\mathbf{p})+C_{0}(\mathbf{p})\right]\left[E_{\mathbf{p}}+\frac{m}{E_{\mathbf{p}}} \Sigma_{0}(\mathbf{p})\right] \\
& \simeq E_{\mathbf{p}}+\frac{m}{E_{\mathbf{p}}} \Sigma_{0}(\mathbf{p}) \simeq \epsilon(\mathbf{p}) .
\end{aligned}
$$

After some algebra the following first-order expressions are obtained:

$$
\begin{aligned}
& \sqrt{\frac{\tilde{E}+\tilde{m}}{2 \tilde{E}}} \simeq \sqrt{\frac{m+E_{\mathbf{p}}}{2 E_{\mathbf{p}}}}\left(1+\frac{E_{\mathbf{p}}-m}{2 E_{\mathbf{p}}} \frac{\Sigma_{0}}{E_{\mathbf{p}}}\right), \\
& \frac{1}{\tilde{E}+\tilde{m}} \simeq \frac{1}{m+E_{\mathbf{p}}}\left(1-\frac{\Sigma_{0}}{E_{\mathbf{p}}}\right) .
\end{aligned}
$$

Inserting Eqs. (88) and (89) into the renormalized spinor in Eq. (85) we get

$$
\begin{aligned}
& \sqrt{\frac{\tilde{m}(\mathbf{p})}{\tilde{E}(\mathbf{p})}} u(\mathbf{p}, \tilde{m}(\mathbf{p})) \simeq \sqrt{\frac{m+E_{\mathbf{p}}}{2 E_{\mathbf{p}}}}\left[1+\frac{E_{\mathbf{p}}-m}{2 E_{\mathbf{p}}} \frac{\Sigma_{0}}{E_{\mathbf{p}}}\right]\left[\begin{array}{c}
\chi \\
\left.\frac{\boldsymbol{\sigma} \cdot \mathbf{p}}{m+E_{\mathbf{p}}}\left(1-\frac{\Sigma_{0}}{E_{\mathbf{p}}}\right) \chi\right]
\end{array}\right] \\
& \simeq \sqrt{\frac{m}{E_{\mathbf{p}}}} u(\mathbf{p})+\frac{\Sigma_{0}}{E_{\mathbf{p}}} \sqrt{\frac{m}{E_{\mathbf{p}}}} \sqrt{\frac{m+E_{\mathbf{p}}}{2 m}}\left[\begin{array}{c}
\frac{E_{\mathbf{p}}-m}{2 E_{\mathbf{p}}} \chi \\
-\frac{E_{\mathbf{p}}+m}{2 E_{\mathbf{p}}} \frac{\boldsymbol{\sigma} \cdot \mathbf{p}}{m+E_{\mathbf{p}}} \chi
\end{array}\right] .
\end{aligned}
$$


Since

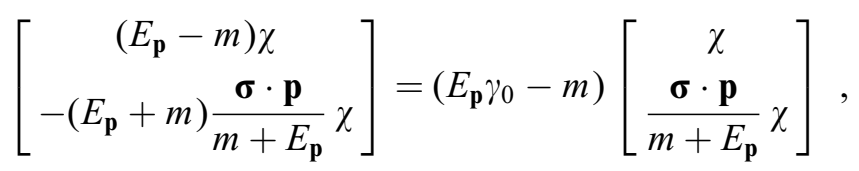

the first-order (in $f^{2} / m_{\pi}^{2}$ ) renormalized spinor can be cast in the form

$$
\sqrt{\frac{\tilde{m}(\mathbf{p})}{\tilde{E}(\mathbf{p})}} u(\mathbf{p}, \tilde{m}(\mathbf{p})) \simeq \sqrt{\frac{m}{E_{\mathbf{p}}}}\left[u(\mathbf{p})+\frac{\Sigma_{0}(\mathbf{p})}{E_{\mathbf{p}}} \frac{E_{\mathbf{p}} \gamma_{0}-m}{2 E_{\mathbf{p}}} u(\mathbf{p})\right] .
$$

The above expansion transparently displays the effect of the self-energy on the free spinor $u(\mathbf{p})$. Indeed the second term in the square brackets of Eq. (92) corresponds to a negative-energy component with momentum p. In fact, the Dirac equation for a positive-energy spinor is given by

$$
(\mathbf{p} \cdot \boldsymbol{\gamma}+m) u(\mathbf{p})=E_{\mathbf{p}} \gamma_{0} u(\mathbf{p}) \quad \text { with } E_{\mathbf{p}}>0 .
$$

Now if we apply the operator $(\mathbf{p} \cdot \boldsymbol{\gamma}+m)$ to the spinor $\left(E_{\mathbf{p}} \gamma_{0}-m\right) u(\mathbf{p})$, we obtain

$$
\begin{aligned}
(\mathbf{p} \cdot \boldsymbol{\gamma}+m)\left(E_{\mathbf{p}} \gamma_{0}-m\right) u(\mathbf{p}) & =\mathbf{p} \cdot \gamma\left(E_{\mathbf{p}} \gamma_{0}-m\right) u(\mathbf{p})+m\left(E_{\mathbf{p}} \gamma_{0}-m\right) u(\mathbf{p}) \\
& =\left(-E_{\mathbf{p}} \gamma_{0}-m\right) \mathbf{p} \cdot \boldsymbol{\gamma} u(\mathbf{p})+m\left(E_{\mathbf{p}} \gamma_{0}-m\right) u(\mathbf{p}) \\
& =\left(-E_{\mathbf{p}} \gamma_{0}-m\right)\left(E_{\mathbf{p}} \gamma_{0}-m\right) u(\mathbf{p})+m\left(E_{\mathbf{p}} \gamma_{0}-m\right) u(\mathbf{p}) \\
& =-E_{\mathbf{p}} \gamma_{0}\left(E_{\mathbf{p}} \gamma_{0}-m\right) u(\mathbf{p}) .
\end{aligned}
$$

Hence $\left(E_{\mathbf{p}} \gamma_{0}-m\right) u$ is an eigenvector of the free Dirac Hamiltonian with eigenvalue $-E_{\mathbf{p}}$. Therefore the operator $E_{\mathbf{p}} \gamma_{0}-m$ transforms a positive-energy spinor $u(\mathbf{p})$ into a negative-energy one.

Moreover, it is useful to write down the correction to the free spinor (see Eq. (92)) in an alternative form. Using the identity in Eq. (94) we can write

$$
(P-m)\left(E_{\mathbf{p}} \gamma_{0}-m\right) u(\mathbf{p})=2 E_{\mathbf{p}} \gamma_{0}\left(E_{\mathbf{p}} \gamma_{0}-m\right) u(\mathbf{p}) .
$$

Multiplying by $\left[2 E_{\mathbf{p}}(P-m)\right]^{-1}$ we then obtain

$$
\frac{E_{\mathbf{p}} \gamma_{0}-m}{2 E_{\mathbf{p}}} u(\mathbf{p})=\frac{1}{P-m} \gamma_{0}\left(E_{\mathbf{p}} \gamma_{0}-m\right) u(\mathbf{p}) .
$$

Hence the second term in the square brackets on the right-hand side of Eq. (92) can be recast in the form

$$
\begin{aligned}
\frac{\Sigma_{0}}{E_{\mathbf{p}}} \frac{E_{\mathbf{p}} \gamma_{0}-m}{2 E_{\mathbf{p}}} u(\mathbf{p}) & =\frac{\Sigma_{0}}{E_{\mathbf{p}}} \frac{1}{P-m} \gamma_{0}\left(E_{\mathbf{p}} \gamma_{0}-m\right) u(\mathbf{p})=\frac{1}{P-m}\left(1-\frac{m}{E_{\mathbf{p}}} \gamma_{0}\right) \Sigma(\mathbf{p}) u(\mathbf{p}) \\
& =S_{\mathrm{F}}(\mathbf{p})\left(1-\frac{m}{E_{\mathbf{p}}} \gamma_{0}\right) \Sigma(\mathbf{p}) u(\mathbf{p}) .
\end{aligned}
$$

The first term in Eq. (97), $S_{\mathrm{F}}(\mathbf{p}) \Sigma(\mathbf{p}) u(\mathbf{p})$, corresponds to the one that enters in the original (divergent) self-energy current for a nucleon on-shell (Eq. (39)). The subtracted term, with the factor 
$\left(m / E_{\mathbf{p}}\right) \gamma_{0}$ inserted between the propagator and the self-energy, cancels the divergence and yields a finite result. Thus it can be viewed as a "recipe" to renormalize the self-energy current.

We turn now to an expansion of the field-strength renormalization function defined in Eq. (66). For this purpose we use Eqs. (86) and (87), obtaining

$$
Z_{2}(\mathbf{p}) \simeq\left[1+B_{0}(\mathbf{p})+\frac{m^{2}}{E_{\mathbf{p}}} \frac{\partial A}{\partial p_{0}}+E_{\mathbf{p}} \frac{\partial B}{\partial p_{0}}-\frac{\mathbf{p}^{2}}{E_{\mathbf{p}}} \frac{\partial C}{\partial p_{0}}\right]_{p_{0}=E_{\mathbf{p}}},
$$

which implies that

$$
\sqrt{Z_{2}(\mathbf{p})} \simeq 1+\frac{1}{2} \alpha(\mathbf{p})
$$

with

$$
\alpha(\mathbf{p}) \equiv B_{0}(\mathbf{p})+\left[\frac{m^{2}}{E_{\mathbf{p}}} \frac{\partial A}{\partial p_{0}}+E_{\mathbf{p}} \frac{\partial B}{\partial p_{0}}-\left.\frac{\mathbf{p}^{2}}{E_{\mathbf{p}}} \frac{\partial C}{\partial p_{0}}\right|_{p_{0}=E_{\mathbf{p}}} .\right.
$$

Hence, collecting the above results and inserting them into Eq. (70), we get to first order

$$
\sqrt{\frac{\tilde{m}(\mathbf{p})}{\widetilde{p_{0}}(\mathbf{p})}} \phi(\mathbf{p}) \simeq \sqrt{\frac{m}{E_{\mathbf{p}}}}\left[u(\mathbf{p})+\frac{\Sigma_{0}}{E_{\mathbf{p}}} \frac{E_{\mathbf{p}} \gamma_{0}-m}{2 E_{\mathbf{p}}} u(\mathbf{p})+\frac{1}{2} \alpha(\mathbf{p}) u(\mathbf{p})\right] .
$$

Thus, within the OPE approach the renormalized HF spinors in the nuclear medium are characterized by two new elements with respect to the bare $u(\mathbf{p})$ : the term $\Sigma_{0} / E_{\mathbf{p}}\left[\left(E_{\mathbf{p}} \gamma_{0}-m\right) / 2 E_{\mathbf{p}}\right] u(\mathbf{p})$ introduces negative-energy components in the wave function, while the term $\frac{1}{2} \alpha(\mathbf{p}) u(\mathbf{p})$ comes from the field-strength renormalization which modifies the occupation number of the single-particle states.

Using the above expressions for the renormalized spinors, we now expand the renormalized one-body current matrix element to first order in $f^{2} / m_{\pi}^{2}$, getting

$$
\begin{aligned}
\sqrt{\frac{\tilde{m}(\mathbf{p})}{\tilde{E}(\mathbf{p})}} \sqrt{\frac{\tilde{m}(\mathbf{h})}{\tilde{E}(\mathbf{h})}} j_{\mathrm{HF}}^{\mu}(\mathbf{p}, \mathbf{h}) \simeq & \sqrt{\frac{m}{E_{\mathbf{p}}} \frac{m}{E_{\mathbf{h}}}} \bar{u}(\mathbf{p})\left[\Gamma^{\mu}+\Gamma^{\mu} \frac{\Sigma_{0}(\mathbf{h})}{E_{\mathbf{h}}} \frac{E_{\mathbf{h}} \gamma_{0}-m}{2 E_{\mathbf{h}}}+\frac{\alpha(\mathbf{h})}{2} \Gamma^{\mu}\right. \\
& \left.+\frac{\Sigma_{0}(\mathbf{p})}{E_{\mathbf{p}}} \frac{E_{\mathbf{p}} \gamma_{0}-m}{2 E_{\mathbf{p}}} \Gamma^{\mu}+\frac{\alpha(\mathbf{p})}{2} \Gamma^{\mu}\right] u(\mathbf{h}) \\
\equiv & \frac{m}{\sqrt{E_{\mathbf{p}} E_{\mathbf{h}}}}\left[j_{\mathrm{OB}}^{\mu}(\mathbf{p}, \mathbf{h})+j_{\mathrm{RSE}}^{\mu}(\mathbf{p}, \mathbf{h})\right]
\end{aligned}
$$

In Eq. (102) the term $j_{\mathrm{OB}}^{\mu}$ represents the usual one-body current matrix element evaluated with free spinors, i.e.,

$$
j_{\mathrm{OB}}^{\mu}(\mathbf{p}, \mathbf{h})=\bar{u}(\mathbf{p}) \Gamma^{\mu}(Q) u(\mathbf{h}),
$$

whereas $j_{\mathrm{RSE}}^{\mu}$ is a new renormalized self-energy (RSE) current matrix element that includes the effects of the renormalization of the spinors. It can be decomposed according to

$$
j_{\mathrm{RSE}}^{\mu}(\mathbf{p}, \mathbf{h})=j_{\mathrm{RSE} 1}^{\mu}(\mathbf{p}, \mathbf{h})+j_{\mathrm{RSE} 2}^{\mu}(\mathbf{p}, \mathbf{h}),
$$


where $j_{\mathrm{RSE} 1}^{\mu}$ embodies the correction arising from the new spinor solution of the Dirac equation in the medium and $j_{\mathrm{RSE} 2}^{\mu}$ the one stemming from the field-strength renormalization function $\sqrt{Z_{2}}$ in the medium. Their expressions are the following:

$$
\begin{aligned}
& j_{\mathrm{RSE} 1}^{\mu}(\mathbf{p}, \mathbf{h})=\bar{u}(\mathbf{p})\left[\Gamma^{\mu} \frac{\Sigma_{0}(\mathbf{h})}{E_{\mathbf{h}}} \frac{E_{\mathbf{h}} \gamma_{0}-m}{2 E_{\mathbf{h}}}+\frac{\Sigma_{0}(\mathbf{p})}{E_{\mathbf{p}}} \frac{E_{\mathbf{p}} \gamma_{0}-m}{2 E_{\mathbf{p}}} \Gamma^{\mu}\right] u(\mathbf{h}), \\
& j_{\mathrm{RSE} 2}^{\mu}(\mathbf{p}, \mathbf{h})=\left[\frac{\alpha(\mathbf{h})+\alpha(\mathbf{p})}{2}\right] j_{\mathrm{OB}}^{\mu}(\mathbf{p}, \mathbf{h}) .
\end{aligned}
$$

\subsection{Gauge invariance of the theory}

A crucial feature of the present theory is that the hadronic tensor, computed either through the $\mathrm{p}-\mathrm{h}$ matrix elements or through the polarization propagator, is gauge invariant. This may be somewhat surprising because, as shown in Appendix B (see also [19]), current conservation is already obtained at the level of the MEC and correlation $\mathrm{p}-\mathrm{h}$ matrix elements: hence the one-body current $\mathrm{p}-\mathrm{h}$ matrix element also has to be independently conserved. This however occurs only in zeroth order of perturbation theory. To be dealt with properly, the situation clearly requires the renormalization of the $\mathrm{p}-\mathrm{h}$ energies and of the Dirac spinors (see previous sections). Only then does it become possible to set up a renormalized SE current which leads to a hadronic tensor coinciding with the one obtained through the polarization propagator [19].

As shown in the previous section, the renormalized HF current matrix element, expanded to first order in $f^{2} / m_{\pi}^{2}$, has been split into the usual one-body current and into a new renormalized self-energy current. In order to be consistent with the OPE model, we should add the contribution of the seagull, pion-in-flight and vertex correlation currents corresponding to the diagrams shown in Fig. 2a-e. We point out once more that the self-energy diagrams (f) and (g), of Fig. 2, corresponding to insertions in external legs, should be disregarded in computing amplitudes (or currents) in perturbation theory. Rather, their contributions should be taken into account via renormalized energies and spinors as solutions of the relativistic HF equations. We have expressed the latter, to first order in $f^{2} / m_{\pi}^{2}$, in the form of a new current operator (denoted as RSE current).

Then the total current in our model reads

$$
j^{\mu}(\mathbf{p}, \mathbf{h})=j_{\mathrm{OB}}^{\mu}(\mathbf{p}, \mathbf{h})+j_{\mathrm{OPE}}^{\mu}(\mathbf{p}, \mathbf{h}),
$$

where $j_{\mathrm{OPE}}^{\mu}$ embodies the seagull, pion-in-flight, vertex correlation and renormalized self-energy currents, namely

$$
j_{\mathrm{OPE}}^{\mu}=j_{\mathrm{s}}^{\mu}+j_{\mathrm{p}}^{\mu}+j_{\mathrm{VC}}^{\mu}+j_{\mathrm{RSE}}^{\mu} .
$$

In what follows we shall prove the gauge invariance of this current to first order in $f^{2} / m_{\pi}^{2}$. In so-doing it is crucial to take into account not only the full current in Eqs. (107) and (108), but also the first-order correction to the energy of the particles and holes due to the self-energy interaction in Eq. (84). In other words, for a given momentum transfer $\mathbf{q}=\mathbf{p}-\mathbf{h}$, the energy transfer should be computed as the difference between the particle and hole HF energies and not using the free values 
$E_{\mathbf{p}}$ and $E_{\mathbf{h}}$. Thus the energy transfer is

$$
\omega_{\mathrm{HF}}=E_{\mathbf{p}}-E_{\mathbf{h}}+\frac{m}{E_{\mathbf{p}}} \Sigma_{0}(\mathbf{p})-\frac{m}{E_{\mathbf{h}}} \Sigma_{0}(\mathbf{h})
$$

and the associated four-momentum transfer is $Q_{\mathrm{HF}}^{\mu}=\left(\omega_{\mathrm{HF}}, \mathbf{q}\right)$. To make the following discussion clearer we denote with $Q_{\mathrm{HF}}$ the $\mathrm{HF}$ four-momentum and with $\omega_{\mathrm{HF}}$ the HF energy transfer, to distinguish them from the on-shell values $Q$ and $\omega$.

\subsubsection{Divergence of the one-body current}

The divergence of the zeroth-order one-body current computed using the HF four-momentum transfer $Q_{\mathrm{HF}}$ is given by

$$
Q_{\mathrm{HF}, \mu} j_{\mathrm{OB}}^{\mu}(\mathbf{p}, \mathbf{h})=\bar{u}(\mathbf{p}) Q_{\mathrm{HF}, \mu} \Gamma^{\mu}\left(Q_{\mathrm{HF}}\right) u(\mathbf{h})=\bar{u}(\mathbf{p}) F_{1}\left(Q_{\mathrm{HF}}\right) Q_{\mathrm{HF}} u(\mathbf{h}),
$$

where the nucleon vertex $\Gamma^{\mu}\left(Q_{\mathrm{HF}}\right)$ is also evaluated at the momentum transfer $Q_{\mathrm{HF}}$. Because of $\bar{u}(\mathbf{p}) Q u(\mathbf{h})=0$, only the first-order contribution arising from the self-energy correction survives, namely

$$
Q_{\mathrm{HF}, \mu} j_{\mathrm{OB}}^{\mu}(\mathbf{p}, \mathbf{h})=\bar{u}(\mathbf{p}) F_{1}(Q)\left[\frac{m}{E_{\mathbf{p}}} \Sigma_{0}(\mathbf{p})-\frac{m}{E_{\mathbf{h}}} \Sigma_{0}(\mathbf{h})\right] \gamma_{0} u(\mathbf{h}) .
$$

In the above, the Dirac form factor $F_{1}$ is computed at the unperturbed value $Q^{\mu}$, since we disregard second-order contributions. Note that the one-body current itself is not gauge invariant-its divergence yields a first-order term which turns out to be essential for the gauge invariance of the full current, as we shall see below.

\subsubsection{Divergence of the $M E C$}

The seagull and pionic 1p-1h currents given in Eqs. (25) and (26) are already of first order in $f^{2} / m_{\pi}^{2}$; thus in computing their divergence we use the unperturbed value of the energy transfer, neglecting a term of order $O\left(f^{4} / m_{\pi}^{4}\right)$. Using the free Dirac equation and exploiting the kinematics we obtain

$$
\begin{aligned}
& Q_{\mu} j_{\mathrm{s}}^{\mu}(\mathbf{p}, \mathbf{h}) \\
& \quad=-\frac{f^{2}}{V m_{\pi}^{2}} F_{1}^{\mathrm{V}} \mathrm{i} \varepsilon_{3 a b} \bar{u}(\mathbf{p}) \tau_{a} \tau_{b} \sum_{\mathbf{k} \leqslant k_{\mathrm{F}}} \frac{m}{E_{\mathbf{k}}}\left\{\frac{2(K \cdot P-m K)}{(P-K)^{2}-m_{\pi}^{2}}-\frac{2(K \cdot H-m K)}{(K-H)^{2}-m_{\pi}^{2}}\right\} u(\mathbf{h}), \\
& Q_{\mu} j_{\mathrm{p}}^{\mu}(\mathbf{p}, \mathbf{h}) \\
& \quad=-\frac{f^{2}}{V m_{\pi}^{2}} F_{1}^{\mathrm{V}} \mathrm{i} \varepsilon_{3 a b} \bar{u}(\mathbf{p}) \tau_{a} \tau_{b} \sum_{\mathbf{k} \leqslant k_{\mathrm{F}}} \frac{m}{E_{\mathbf{k}}}\left\{\frac{2 m(K-m)}{(P-K)^{2}-m_{\pi}^{2}}-\frac{2 m(K-m)}{(K-H)^{2}-m_{\pi}^{2}}\right\} u(\mathbf{h}) .
\end{aligned}
$$

In deriving these equations we have used the relations $Q_{\mu}(Q+2 H-2 K)^{\mu}=-2 K \cdot Q$ and

$$
\frac{1}{(K-H)^{2}-m_{\pi}^{2}}-\frac{1}{(P-K)^{2}-m_{\pi}^{2}}=\frac{-2 P \cdot Q}{\left[(K-H)^{2}-m_{\pi}^{2}\right]\left[(P-K)^{2}-m_{\pi}^{2}\right]} .
$$


Upon addition of Eqs. (112) and (113) the terms containing $K$ cancel, leaving for the total divergence of the seagull and pion-in-flight the expression

$$
\begin{aligned}
& Q_{\mu}\left(j_{\mathrm{s}}^{\mu}+j_{\mathrm{p}}^{\mu}\right) \\
& \quad=-\frac{f^{2}}{V m_{\pi}^{2}} F_{1}^{\mathrm{V}} \mathrm{i} \varepsilon_{3 a b} \bar{u}(\mathbf{p}) \tau_{a} \tau_{b} \sum_{\mathbf{k} \leqslant k \mathrm{~F}} \frac{m}{E_{\mathbf{k}}}\left\{\frac{2\left(K \cdot H-m^{2}\right)}{(K-H)^{2}-m_{\pi}^{2}}-\frac{2\left(K \cdot P-m^{2}\right)}{(P-K)^{2}-m_{\pi}^{2}}\right\} u(\mathbf{h}),
\end{aligned}
$$

which can be further simplified by exploiting the self-energy of Eq. (43) for on-shell momenta. One finally obtains

$$
Q_{\mu}\left(j_{\mathrm{s}}^{\mu}+j_{\mathrm{p}}^{\mu}\right)=\frac{\mathrm{i}}{3} F_{1}^{\mathrm{V}} \varepsilon_{3 a b} \bar{u}(\mathbf{p}) \tau_{a} \tau_{b}[\Sigma(\mathbf{p})-\Sigma(\mathbf{h})] u(\mathbf{h}) .
$$

\subsubsection{Divergence of the vertex correlation current}

Starting from the $1 \mathrm{p}-1 \mathrm{~h}$ matrix element of the VC current in Eq. (27) and applying the Dirac equation, we get

$$
\begin{aligned}
Q_{\mu} j_{\mathrm{VC}}^{\mu}(\mathbf{p}, \mathbf{h})= & \frac{f^{2}}{V m_{\pi}^{2}} \bar{u}(\mathbf{p}) \tau_{a} F_{1} \tau_{a} \sum_{\mathbf{k} \leqslant k_{\mathrm{F}}} \frac{1}{2 E_{\mathbf{k}}} \gamma_{5}(P-K) \frac{K+m}{(P-K)^{2}-m_{\pi}^{2}} \gamma_{5}(P-K) u(\mathbf{h}) \\
& -\frac{f^{2}}{V m_{\pi}^{2}} \bar{u}(\mathbf{p}) \tau_{a} F_{1} \tau_{a} \sum_{\mathbf{k} \leqslant k_{\mathrm{F}}} \frac{1}{2 E_{\mathbf{k}}} \gamma_{5}(K-H) \frac{K+m}{(K-H)^{2}-m_{\pi}^{2}} \gamma_{5}(K-H) u(\mathbf{h}),
\end{aligned}
$$

where we recognize again the expression of the self-energy matrix in Eq. (11). Since the Dirac form factor can be split into an isoscalar and an isovector component according to

$$
F_{1}=\frac{1}{2}\left(F_{1}^{\mathrm{S}}+F_{1}^{\mathrm{V}} \tau_{3}\right),
$$

which yields

$$
\tau_{a} F_{1} \tau_{a}=3 F_{1}+\mathrm{i} F_{1}^{\mathrm{V}} \varepsilon_{3 a b} \tau_{a} \tau_{b},
$$

the divergence of the $\mathrm{VC}$ current written in terms of the self-energy function reads

$$
Q_{\mu} j_{\mathrm{VC}}^{\mu}(\mathbf{p}, \mathbf{h})=\bar{u}(\mathbf{p})\left(F_{1}+\frac{\mathrm{i}}{3} F_{1}^{\mathrm{V}} \varepsilon_{3 a b} \tau_{a} \tau_{b}\right)[\Sigma(\mathbf{h})-\Sigma(\mathbf{p})] u(\mathbf{h}) .
$$

Comparing this result with Eq. (116) we note that the term above containing $\epsilon_{3 a b} \tau_{a} \tau_{b}$ cancels with the MEC contribution. Hence

$$
Q_{\mu}\left[j_{\mathrm{MEC}}^{\mu}(\mathbf{p}, \mathbf{h})+j_{\mathrm{VC}}^{\mu}(\mathbf{p}, \mathbf{h})\right]=\bar{u}(\mathbf{p}) F_{1}[\Sigma(\mathbf{h})-\Sigma(\mathbf{p})] u(\mathbf{h}) .
$$

The above relation just expresses the Ward-Takahashi identity [46] relating the full vertex correction, namely MEC plus VC (Fig. 2a-e), to the self-energy matrix element.

\subsubsection{Divergence of the RSE current}

Finally we compute the divergence of the RSE current defined in Eqs. (104)-(106). For this purpose we first note that the divergence of $j_{\mathrm{RSE} 2}^{\mu}$ vanishes to first order because it is proportional 
to the OB current. Hence we write

$$
Q_{\mu} j_{\mathrm{RSE}}^{\mu}(\mathbf{p}, \mathbf{h})=\bar{u}(\mathbf{p})\left[F_{1} Q \frac{\Sigma_{0}(\mathbf{h})}{E_{\mathbf{h}}} \frac{E_{\mathbf{h}} \gamma_{0}-m}{2 E_{\mathbf{h}}}+\frac{\Sigma_{0}(\mathbf{p})}{E_{\mathbf{p}}} \frac{E_{\mathbf{p}} \gamma_{0}-m}{2 E_{\mathbf{p}}} F_{1} \mathscr{Q}\right] u(\mathbf{h}) .
$$

Using the relation $\bar{u}(\mathbf{p}) Q u(\mathbf{h})=0$ and

$$
\begin{aligned}
& \bar{u}(\mathbf{p}) Q_{0} u(\mathbf{h})=\bar{u}(\mathbf{p}) 2\left(m \gamma_{0}-E_{\mathbf{h}}\right) u(\mathbf{h}), \\
& \bar{u}(\mathbf{p}) \gamma_{0} Q u(\mathbf{h})=\bar{u}(\mathbf{p}) 2\left(E_{\mathbf{p}}-m \gamma_{0}\right) u(\mathbf{h}),
\end{aligned}
$$

it is straightforward to obtain

$$
Q_{\mu} j_{\mathrm{RSE}}^{\mu}(\mathbf{p}, \mathbf{h})=\bar{u}(\mathbf{p}) F_{1}[\Sigma(\mathbf{p})-\Sigma(\mathbf{h})] u(\mathbf{h})+\bar{u}(\mathbf{p}) F_{1}\left[\frac{m}{E_{\mathbf{h}}} \Sigma_{0}(\mathbf{h})-\frac{m}{E_{\mathbf{p}}} \Sigma_{0}(\mathbf{p})\right] u(\mathbf{h}) .
$$

Remarkably the first term of this equation cancels with the divergence of the MEC plus the VC current, given by the Ward-Takahashi identity in Eq. (121), whereas the second term cancels with the divergence of the $\mathrm{OB}$ current in Eq. (111). We have thus proven that, within the present model up to first order in $f^{2} / m_{\pi}^{2}$, the total current in Eq. (107) satisfies the continuity equation, namely

$$
Q_{\mathrm{HF}, \mu}\left(j_{\mathrm{OB}}^{\mu}+j_{\mathrm{MEC}}^{\mu}+j_{\mathrm{VC}}^{\mu}+j_{\mathrm{RSE}}^{\mu}\right)=0 \text {. }
$$

\subsection{Nuclear hadronic tensor and electromagnetic response functions}

In this section we compute the electromagnetic inclusive response functions for one-particle emission reactions within the RFG model. As discussed in previous sections, the $\mathrm{p}-\mathrm{h}$ matrix elements corresponding to the different pionic diagrams are all well defined except for the self-energy term which diverges, and consequently needs to be renormalized. In what follows, we evaluate the hadronic tensor starting from the current $\mathrm{p}-\mathrm{h}$ matrix elements in the case of the one-body, MEC and vertex correlation diagrams. These are shown diagrammatically in Fig. 6. On the contrary, for the self-energy diagrams we calculate the hadronic tensor in two at first sight different ways: on the one hand, from the polarization propagator $\Pi^{\mu \nu}$ (see Appendix C), and on the other, using the renormalized, well-defined, SE $\mathrm{p}-\mathrm{h}$ matrix elements. In Appendix D we prove that the two formalisms are equivalent.

The formalism of the nuclear hadronic tensor set up with the $\mathrm{p}-\mathrm{h}$ matrix elements has been presented in detail, within the RFG model, in previous papers [24,51]. Hence, here we simply summarize the results needed for later discussions. Before starting the analysis of pionic contributions, we recall the analytic expressions for the one-body (OB), leading-order electromagnetic responses of the RFG (see, for example, [35,41] for details):

$$
R^{\mathrm{L}, \mathrm{T}}(q, \omega)=R_{0}(q, \omega)\left[U_{\mathrm{p}}^{\mathrm{L}, \mathrm{T}}(q, \omega)+U_{\mathrm{n}}^{\mathrm{L}, \mathrm{T}}(q, \omega)\right],
$$

where $\mathrm{p}$ and $\mathrm{n}$ refer to protons and neutrons, respectively, and, for $Z=N$,

$$
R_{0}(q, \omega) \equiv \frac{3 Z}{4 m \kappa \eta_{\mathrm{F}}^{3}}\left(\varepsilon_{\mathrm{F}}-\varepsilon_{0}\right) \theta\left(\varepsilon_{\mathrm{F}}-\varepsilon_{0}\right)
$$




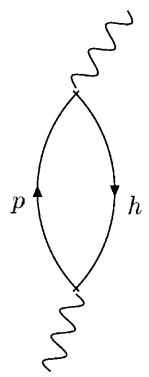

(a)

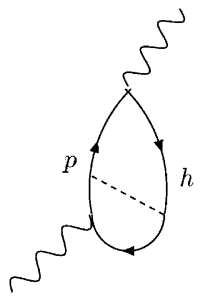

(e)

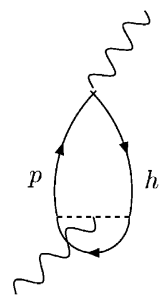

(b)

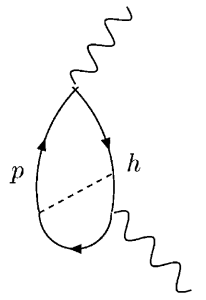

(f)

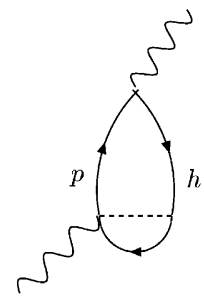

(c)

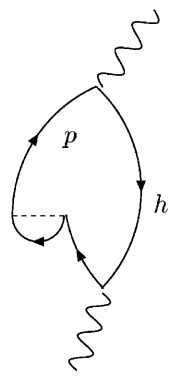

(g)

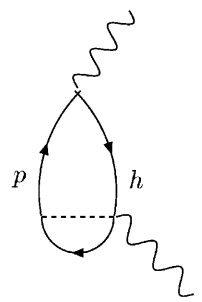

(d)

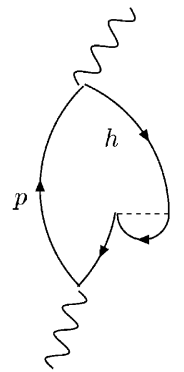

(h)

Fig. 6. Feynman diagrams of the free (a) and first-order pion-in-flight (b), seagull (c and d), vertex correlation (e and f) and self-energy $(\mathrm{g}$ and $\mathrm{h})$ polarization propagator.

with

$$
\varepsilon_{0}=\max \left\{\varepsilon_{\mathrm{F}}-2 \lambda, \kappa \sqrt{1+\frac{1}{\tau}}-\lambda\right\} .
$$

In the above the usual dimensionless variables

$$
\lambda=\frac{\omega}{2 m}, \quad \tau=\frac{\left|Q^{2}\right|}{4 m^{2}}, \quad \kappa=\frac{q}{2 m}, \quad \eta_{\mathrm{F}}=\frac{k_{\mathrm{F}}}{m}, \quad \varepsilon_{\mathrm{F}}=\frac{E_{\mathrm{F}}}{m}
$$

have been introduced and $E_{\mathrm{F}}=\sqrt{k_{\mathrm{F}}^{2}+m^{2}}$ is the Fermi energy. The functions $U^{\mathrm{L}, \mathrm{T}}$ in Eq. (127) are

$$
\begin{aligned}
& U_{\mathrm{p}(\mathrm{n})}^{\mathrm{L}}(q, \omega)=\frac{\kappa^{2}}{\tau}\left\{G_{\mathrm{Ep}(\mathrm{n})}^{2}+\frac{\Delta}{1+\tau}\left[G_{\mathrm{Ep}(\mathrm{n})}^{2}+\tau G_{\mathrm{Mp}(\mathrm{n})}^{2}\right]\right\}, \\
& U_{\mathrm{p}(\mathrm{n})}^{\mathrm{T}}(q, \omega)=2 \tau G_{\mathrm{Mp}(\mathrm{n})}^{2}+\frac{\Delta}{1+\tau}\left[G_{\mathrm{Ep}(\mathrm{n})}^{2}+\tau G_{\mathrm{Mp}(\mathrm{n})}^{2}\right],
\end{aligned}
$$

where

$$
\Delta \equiv \frac{\tau}{\kappa^{2}}\left[\frac{1}{3}\left(\varepsilon_{\mathrm{F}}^{3}+\varepsilon_{\mathrm{F}} \varepsilon_{0}+\varepsilon_{0}^{2}\right)+\lambda\left(\varepsilon_{\mathrm{F}}+\varepsilon_{0}\right)+\lambda^{2}\right]-(1+\tau) .
$$

\subsubsection{MEC and vertex pionic contributions}

The hadronic tensor that arises from the interference of the single-nucleon, OB current, $j_{\mathrm{OB}}^{\mu}$, with the OPE current $j_{a}^{\mu}$, with $a=\mathrm{s}$ (seagull), p (pion-in-flight) and VC (vertex correlation), is for the 
RFG model with $Z=N$ (see Eq. (193))

$$
W^{\mu v}=\frac{3 Z}{8 \pi k_{\mathrm{F}}^{3} q} \int_{h_{0}}^{k_{\mathrm{F}}} h \mathrm{~d} h\left(\omega+E_{\mathbf{h}}\right) \int_{0}^{2 \pi} \mathrm{d} \phi_{h} \sum_{s_{p}, s_{h}} \frac{m^{2}}{E_{\mathbf{p}} E_{\mathbf{h}}} 2 \operatorname{Re}\left[j_{\mathrm{OB}}^{\mu}(\mathbf{p}, \mathbf{h})^{*} j_{a}^{v}(\mathbf{p}, \mathbf{h})\right],
$$

where $j_{\mathrm{OB}}^{\mu}(\mathbf{p}, \mathbf{h})=\bar{u}(\mathbf{p}) \Gamma^{\mu} u(\mathbf{h})$ is the single-nucleon $\mathrm{p}-\mathrm{h}$ matrix element with $\Gamma^{\mu}$ the electromagnetic nucleon current from Eq. (22) and $j_{a}^{v}(\mathbf{p}, \mathbf{h})$ is the $\mathrm{p}-\mathrm{h}$ matrix element for the seagull, pion-in-flight or vertex current as given in Eqs. (25), (26), and (29) and (30), respectively.

Note that in Eq. (134) the integral over the hole polar angle, $\cos \theta_{h}$, has been performed explicitly by exploiting the energy-conserving $\delta$-function. This fixes the minimum momentum of the hole according to

$$
h_{0}=m \sqrt{\varepsilon_{0}^{2}-1} .
$$

Moreover, the hole three-momentum,

$$
\mathbf{h}=h\left(\sin \theta_{0} \cos \phi_{h}, \sin \theta_{0} \sin \phi_{h}, \cos \theta_{0}\right),
$$

involved in the hadronic tensor must be evaluated for the following specific value of the polar angle:

$$
\cos \theta_{0}=\frac{\lambda \varepsilon-\tau}{\eta \kappa}
$$

with $\eta=h / m$.

The hadronic tensor, as was the case for the current, can be also split into isoscalar and isovector parts, since there is no interference between the two isospin channels.

An important issue relates to the form factor of the $\pi N N$ vertex, $\Gamma_{\pi}$, which incorporates some aspects of the short-range physics affecting the pionic correlations. In all of the above expressions $\Gamma_{\pi}$ has not been explicitly indicated for sake of simplicity. In [19] the analysis of the gauge invariance at the level of the particle-hole channel, performed by deriving the contribution to the continuity equation of the isoscalar and isovector SE, VC and MEC $\mathrm{p}-\mathrm{h}$ matrix elements, is presented. There, it is shown that the SE and VC contributions cancel in the isoscalar channel, in contrast with the non-relativistic result [24], where the SE is by itself gauge invariant. Furthermore, the SE and $\mathrm{VC}$ contribution in the isovector channel is exactly canceled by that of the MEC (seagull and pion-in-flight). It is crucial to recall that the inclusion of $\Gamma_{\pi}$ in the $\mathrm{p}-\mathrm{h}$ current matrix elements is not without consequences in connection with gauge invariance. In fact, in this case, the model is not gauge invariant unless new terms are added to the MEC (see [51-57] for recent work on the restoration of current conservation in model calculations). Lacking a fundamental theory for $\Gamma_{\pi}$, in the calculations reported in this work we use the phenomenological expression

$$
\Gamma_{\pi}(P)=\frac{\Lambda^{2}-m_{\pi}^{2}}{\Lambda^{2}-P^{2}}
$$

with $\Lambda=1.3 \mathrm{GeV}$. As long as the dependence upon $\Lambda$ is not too strong, the gauge invariance of the theory should not be too badly affected. Within a non-relativistic approach for the pion currents, a detailed discussion on the breakdown of the gauge invariance induced by $\Gamma_{\pi}$, and on the dependence of the responses upon the cutoff value can be found in $[51,55]$. 
In $[58,59]$ the effects of the MEC upon the transverse response in a non-relativistic shell model for finite nuclei were studied as a function of the cutoff $\Lambda$.

\subsubsection{Relativistic self-energy responses}

As already discussed in previous sections, a crucial point to be emphasized is that the self-energy $\mathrm{p}-\mathrm{h}$ matrix element, Eq. (39), is divergent. Hence it cannot be used directly in the evaluation of the hadronic tensor. Instead one should use renormalized spinors with the corresponding renormalized energies. Above we have taken account of the effect of renormalization to first order in $f^{2} / m_{\pi}^{2}$ by introducing an extra term in the current: the RSE current defined in Eq. (104). In addition there is also a $O\left(f^{2} / m_{\pi}^{2}\right)$ modification of the energy of the particles, Eq. (84). These two modifications of the free current and energy in turn give a contribution to the hadronic tensor of order $O\left(f^{2} / m_{\pi}^{2}\right)$, which we will refer to as RSE contribution, which is of the same order as the MEC and VC currents and should be included in any consistent calculation to first order in $f^{2} / m_{\pi}^{2}$. In addition this contribution is needed for the gauge invariance of the results.

In what follows we derive the RSE contribution to the nuclear response functions. This RSE contribution should replace the SE Feynman diagrams shown in Fig. $6 \mathrm{~g}$ and h. As a matter of fact, these two diagrams can be computed using the polarization propagator formalism (see Appendix C and Ref. [19]), where one does not need to appeal to renormalization since the SE diagrams are finite in this case. Our goal is to show that the results for the response functions obtained in the two ways coincide, although they stem from different approaches. This is proved in Appendix D. The RSE contribution, therefore, can be identified with the contribution coming from the two diagrams (g) and (h) of Fig. 6.

The one-body hadronic tensor in HF approximation reads

$$
\begin{aligned}
W_{\mathrm{HF}}^{\mu v}(\omega, \mathbf{q})= & V \sum_{s_{p} s_{h}} \sum_{t_{p} t_{h}} \int \frac{\mathrm{d}^{3} h}{(2 \pi)^{3}} \frac{\tilde{m}(\mathbf{p}) \tilde{m}(\mathbf{h})}{\tilde{E}(\mathbf{p}) \tilde{E}(\mathbf{h})} j_{\mathrm{HF}}^{\mu}(\mathbf{p}, \mathbf{h})^{*} j_{\mathrm{HF}}^{v}(\mathbf{p}, \mathbf{h}) \\
& \times \delta(\omega+\epsilon(\mathbf{h})-\epsilon(\mathbf{p})) \theta\left(k_{\mathrm{F}}-h\right),
\end{aligned}
$$

where $\mathbf{p}=\mathbf{h}+\mathbf{q}$ and $j_{\mathrm{HF}}^{\mu}(\mathbf{p}, \mathbf{h})$ is the one-body HF current in Eq. (74) computed using the renormalized HF spinors and HF energies of the particle and the hole.

Next we use the expansions in Eqs. (102) for the current $j_{\mathrm{HF}}$ and (84) for the HF energies. In addition we expand the energy delta function to first order in $f^{2} / m_{\pi}^{2}$ according to

$$
\delta(\omega+\epsilon(\mathbf{h})-\epsilon(\mathbf{p})) \simeq \delta\left(\omega+E_{\mathbf{h}}-E_{\mathbf{p}}\right)+\frac{\mathrm{d} \delta\left(\omega+E_{\mathbf{h}}-E_{\mathbf{p}}\right)}{\mathrm{d} \omega}\left[\frac{m}{E_{\mathbf{h}}} \Sigma_{0}(\mathbf{h})-\frac{m}{E_{\mathbf{p}}} \Sigma_{0}(\mathbf{p})\right] .
$$

Inserting all of these relations into the hadronic tensor and neglecting terms of second order we get for the diagonal elements of the hadronic tensor ${ }^{5}$

$$
W_{\mathrm{HF}}^{\mu \mu}(\omega, \mathbf{q}) \simeq W_{\mathrm{OB}}^{\mu \mu}(\omega, \mathbf{q})+\Delta W_{\mathrm{RSE}}^{\mu \mu}(\omega, \mathbf{q})
$$

\footnotetext{
${ }^{5}$ We only work out the diagonal elements of the hadronic tensor, since these are the ones that contribute to the unpolarized inclusive longitudinal and transverse response functions.
} 
(the summation convention is not in force in Eq. (141)), where $W_{\mathrm{OB}}^{\mu \mu}(\omega, \mathbf{q})$ is the usual OB hadronic tensor of an RFG, i.e.,

$$
W_{\mathrm{OB}}^{\mu \mu}=V \sum_{s_{p} s_{h}} \sum_{t_{p} t_{h}} \int \frac{\mathrm{d}^{3} h}{(2 \pi)^{3}} \frac{m^{2}}{E_{\mathbf{p}} E_{\mathbf{h}}}\left|j_{\mathrm{OB}}^{\mu}(\mathbf{p}, \mathbf{h})\right|^{2} \delta\left(\omega+E_{\mathbf{h}}-E_{\mathbf{p}}\right) \theta\left(k_{\mathrm{F}}-h\right),
$$

and $\Delta W_{\mathrm{RSE}}^{\mu \mu}(\omega, \mathbf{q})$ is the first-order self-energy correction

$$
\begin{aligned}
\Delta W_{\mathrm{RSE}}^{\mu \mu}= & V \sum_{s_{p} s_{h}} \sum_{t_{p} t_{h}} \int \frac{\mathrm{d}^{3} h}{(2 \pi)^{3}} \frac{m^{2}}{E_{\mathbf{p}} E_{\mathbf{h}}}\left\{2 \operatorname{Re} j_{\mathrm{OB}}^{\mu}(\mathbf{p}, \mathbf{h})^{*} j_{\mathrm{RSE}}^{\mu}(\mathbf{p}, \mathbf{h}) \delta\left(\omega+E_{\mathbf{h}}-E_{\mathbf{p}}\right)\right. \\
& \left.+\left|j_{\mathrm{OB}}^{\mu}(\mathbf{p}, \mathbf{h})\right|^{2}\left[\frac{m}{E_{\mathbf{h}}} \Sigma_{0}(\mathbf{h})-\frac{m}{E_{\mathbf{p}}} \Sigma_{0}(\mathbf{p})\right] \frac{\mathrm{d}}{\mathrm{d} \omega} \delta\left(\omega+E_{\mathbf{h}}-E_{\mathbf{p}}\right)\right\} \theta\left(k_{\mathrm{F}}-h\right) .
\end{aligned}
$$

In Eq. (143) the first term corresponds to the interference between the OB and the RSE currents, while the second one, which shifts the allowed kinematical region because of the derivative of the energy delta function, is due to the modification of the nucleon energies in the medium.

Carrying out the spin traces for the single-nucleon current

$$
\sum_{s_{p} s_{h}}\left|j_{\mathrm{OB}}^{\mu}(\mathbf{p}, \mathbf{h})\right|^{2}=\frac{1}{4 m^{2}} \operatorname{Tr}\left\{\Gamma^{\mu}(Q)(H+m) \Gamma^{\mu}(-Q)(P+m)\right\},
$$

we get for the renormalized self-energy response function

$$
\begin{aligned}
\Delta W_{\mathrm{RSE}}^{\mu \mu}= & V \int \frac{\mathrm{d}^{3} h}{(2 \pi)^{3}} \frac{1}{4 E_{\mathbf{p}} E_{\mathbf{h}}} \operatorname{Tr}\left\{\Gamma^{\mu}(Q)\left[\frac{\Sigma_{0}(\mathbf{h})}{E_{\mathbf{h}}} \frac{E_{\mathbf{h}} \gamma_{0}-m}{2 E_{\mathbf{h}}}+\frac{\alpha(\mathbf{h})}{2}\right](H+m) \Gamma^{\mu}(-Q)(P+m)\right. \\
& +\Gamma^{\mu}(Q)(H+m) \Gamma^{\mu}(-Q)(\not P+m)\left[\frac{\Sigma_{0}(\mathbf{p})}{E_{\mathbf{p}}} \frac{E_{\mathbf{p}} \gamma_{0}-m}{2 E_{\mathbf{p}}}+\frac{\alpha(\mathbf{p})}{2}\right] \\
& +\Gamma^{\mu}(Q)(H+m)\left[\frac{\Sigma_{0}(\mathbf{h})}{E_{\mathbf{h}}} \frac{E_{\mathbf{h}} \gamma_{0}-m}{2 E_{\mathbf{h}}}+\frac{\alpha(\mathbf{h})}{2}\right] \Gamma^{\mu}(-Q)(P+m) \\
& \left.+\Gamma^{\mu}(Q)(H+m) \Gamma^{\mu}(-Q)\left[\frac{\Sigma_{0}(\mathbf{p})}{E_{\mathbf{p}}} \frac{E_{\mathbf{p}} \gamma_{0}-m}{2 E_{\mathbf{p}}}+\frac{\alpha(\mathbf{p})}{2}\right](P+m)\right\} \\
& \times \delta\left(\omega+E_{\mathbf{h}}-E_{\mathbf{p}}\right) \theta\left(k_{\mathrm{F}}-h\right) \\
& +V \int \frac{\mathrm{d}^{3} h}{(2 \pi)^{3}} \frac{1}{4 E_{\mathbf{p}} E_{\mathbf{h}}} \operatorname{Tr}\left\{\Gamma^{\mu}(Q)(H+m) \Gamma^{\mu}(-Q)(P+m)\right\} \\
& \times\left(\frac{m}{E_{\mathbf{h}}} \Sigma_{0}(\mathbf{h})-\frac{m}{E_{\mathbf{p}}} \Sigma_{0}(\mathbf{p})\right) \frac{\mathrm{d}}{\mathrm{d} \omega} \delta\left(\omega+E_{\mathbf{h}}-E_{\mathbf{p}}\right) \theta\left(k_{\mathrm{F}}-h\right) .
\end{aligned}
$$

More precisely, one should add two copies of Eq. (145), one with the form factors appropriate to the proton and one to the neutron.

In Appendix $\mathrm{D}$ we show that this contribution to the response function is identical to the one obtained in Appendix $\mathrm{C}$ by computing the imaginary part of the polarization propagator corresponding 
to the two SE diagrams (g) and (h) of Fig. 6. This identity is not trivial: indeed in the case of the polarization propagator the response functions, with the Fock self-energy dressing the particle and the hole lines, are computed by representing the product of two nucleon propagators as the derivative of a single one to deal with the presence of a double pole in the integrand. In the present paper the problem has been solved differently. First the entire perturbative series with Fock self-energy insertions has been summed up and then the result has been expanded to first order, thus obtaining a finite first-order current operator. Because of the equivalence of these two procedures we are confident about the validity of the results we have obtained for the self-energy contribution to the nuclear responses.

\subsection{Analysis of results}

In this section we report the numerical results obtained for the pionic MEC (pion-in-flight and seagull) and for the correlation (vertex and self-energy) contributions to the quasielastic peak (QEP) in the $1 \mathrm{p}-1 \mathrm{~h}$ sector. The calculation is fully relativistic. We have taken $Z=N=20$ and set $k_{\mathrm{F}}=$ $237 \mathrm{MeV} / c$, which is representative of nuclei in the vicinity of ${ }^{40} \mathrm{Ca}$.

The five-dimensional integrations of the MEC and correlation responses implicit in Eq. (134) have been performed numerically. The reliability of the numerical procedure has been proven by checking that the free RFG responses coincide with their analytic expressions (see, e.g., [35]).

\subsection{1. $M E C$}

We start by analyzing the effects introduced by the MEC. These are presented in Figs. 7 and 8 where we show the longitudinal (left panels) and transverse (right panels) response functions versus the transferred energy $\omega$ for four different values of the transferred momentum $q: 0.5,1,2$ and $3 \mathrm{GeV} / c$. First, in Fig. 7 we compare the free RFG responses (dashed) with the responses obtained including the global MEC contribution (solid). As shown, while for the longitudinal responses the MEC are hardly visible, in the T channel they contribute somewhat more, typically by about $5-10 \%$, depending upon $q$ and $\omega$ (see discussion later).

In Fig. 8 we display the separate pion-in-flight (dashed) and seagull (short-dashed) contribution to $R^{\mathrm{L}}$ and $R^{\mathrm{T}}$ for various values of $q$. The total MEC (seagull+pion-in-flight) contribution is also shown (solid line). In the transverse channel (right panels) it appears that the seagull term is always larger than the pion-in-flight term, a dominance that increases with $q$ and reflecting the spin nature of the photon-MEC interaction. Moreover, whereas the pion-in-flight term is always negative, the seagull changes sign with $\omega$, inducing a (mild) softening of the response, i.e., a shift to lower energy. Within the longitudinal channel (left panels), the seagull term, now always negative, also dominates. Note however that the relative difference between the seagull and pion-in-flight contributions is not as large as in the previous case. Moreover, the behavior of the seagull and pion-in-flight terms in the longitudinal channel as $q$ increases displays a different pattern from the one shown in the transverse channel, since for high $q$ the pionic current is not negligible compared with the seagull one.

To complete this discussion we briefly comment on the MEC dependence upon the momentum transfer $q$ and the Fermi momentum $k_{\mathrm{F}}$, associated with scaling of first and second kind, respectively (see [60-62]).

In [19] we have explored in detail the evolution with $q$ of the MEC in the transverse channel (as they are negligible in the longitudinal channel). We have proven that their relative contribution to 

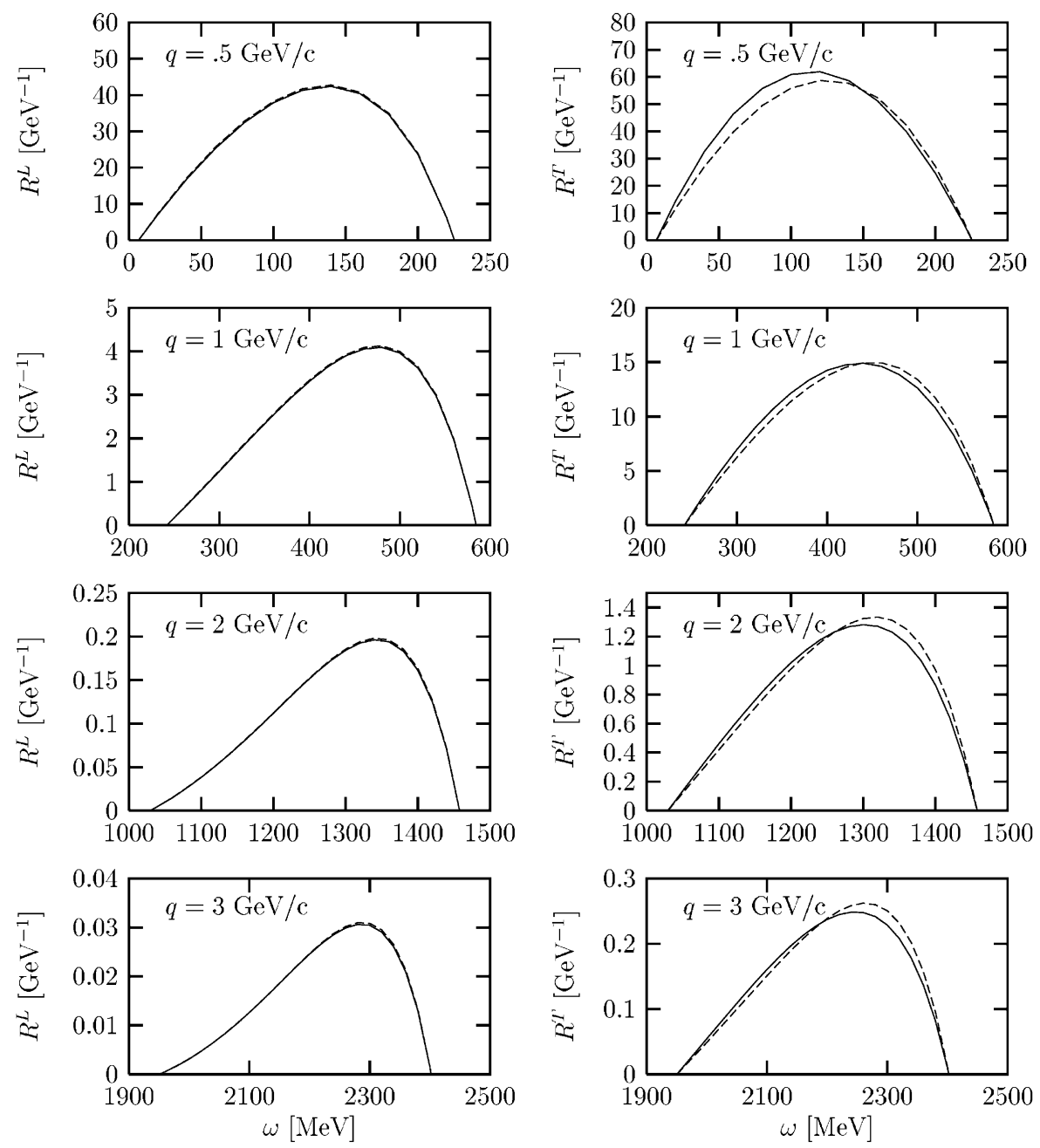

Fig. 7. Longitudinal (left panels) and transverse (right panels) electromagnetic response functions versus $\omega$. Dashed: free RFG; solid: RFG + MEC contribution. Here and in all the figures that follow, unless explicitly indicated, the nucleus is ${ }^{40} \mathrm{Ca}$, corresponding to a Fermi momentum $k_{\mathrm{F}}=237 \mathrm{MeV} / c$.

$R^{\mathrm{T}}$ decreases with $q$, but does not vanish for large values of $q$. In fact, the relative MEC contribution decreases in going from 0.5 to $1 \mathrm{GeV} / c$, but then it rapidly saturates at or slightly above $q=1 \mathrm{GeV} / c$, where its value stabilizes, typically around $10 \%$. Thus, one can conclude that at momentum transfers above $1 \mathrm{GeV} / c$, scaling of the first kind is satisfied for the MEC contributions considered in this work. Moreover, for high $q$ the MEC almost vanish for $\omega$ in the vicinity of the QEP.

A detailed analysis of the $k_{\mathrm{F}}$ dependence of the MEC contribution in the transverse response has also been presented in [19]. The MEC contribution is found to grow with $k_{\mathrm{F}}$, in contrast with the free response which decreases as $k_{\mathrm{F}}^{-1}$. It is also shown that the two-body MEC processes violate the second-kind scaling by roughly three powers of $k_{\mathrm{F}}$. This effect is a rapid function of the Fermi momentum (or equivalently, of the density): for example, if one considers the cases ${ }^{2} \mathrm{H} /{ }^{4} \mathrm{He} /$ heavy 

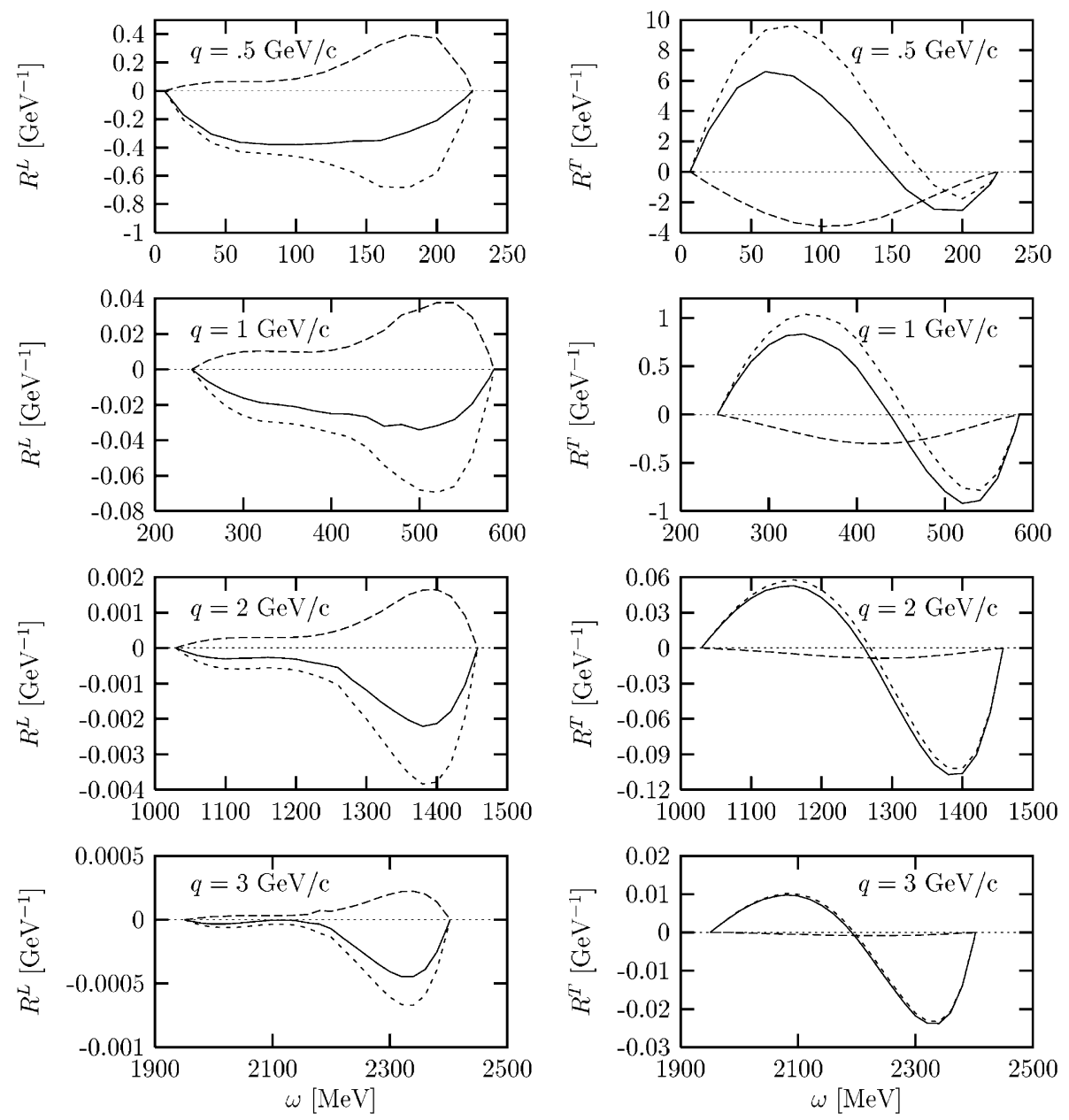

Fig. 8. Separate MEC contribution to the longitudinal (left panels) and transverse (right panels) responses. Dashed: pion-in-flight; short-dashed: seagull and solid: MEC (pion-in-flight+seagull) contribution.

nuclei with Fermi momenta of approximately 55/200/260 MeV/c, respectively, then the $1 \mathrm{p}-1 \mathrm{~h}$ MEC contributions amount to $0.1 \% / 5 \% / 10 \%$ of the total transverse response, respectively (normalizing to $10 \%$ for the heavy nucleus case).

\subsubsection{Correlations}

In Fig. 9 we display the vertex correlation contribution to the longitudinal and transverse responses by comparing the free RFG responses (dashed) with the responses obtained including the VC contribution (solid). As noted, the $\mathrm{VC}$ action, while substantial in both the longitudinal and transverse channel, is actually dominant in the former by roughly a factor of three. This outcome relates to the minor role played by the isoscalar contribution in the transverse response, in turn due to the smallness of the isoscalar magnetic moment. 

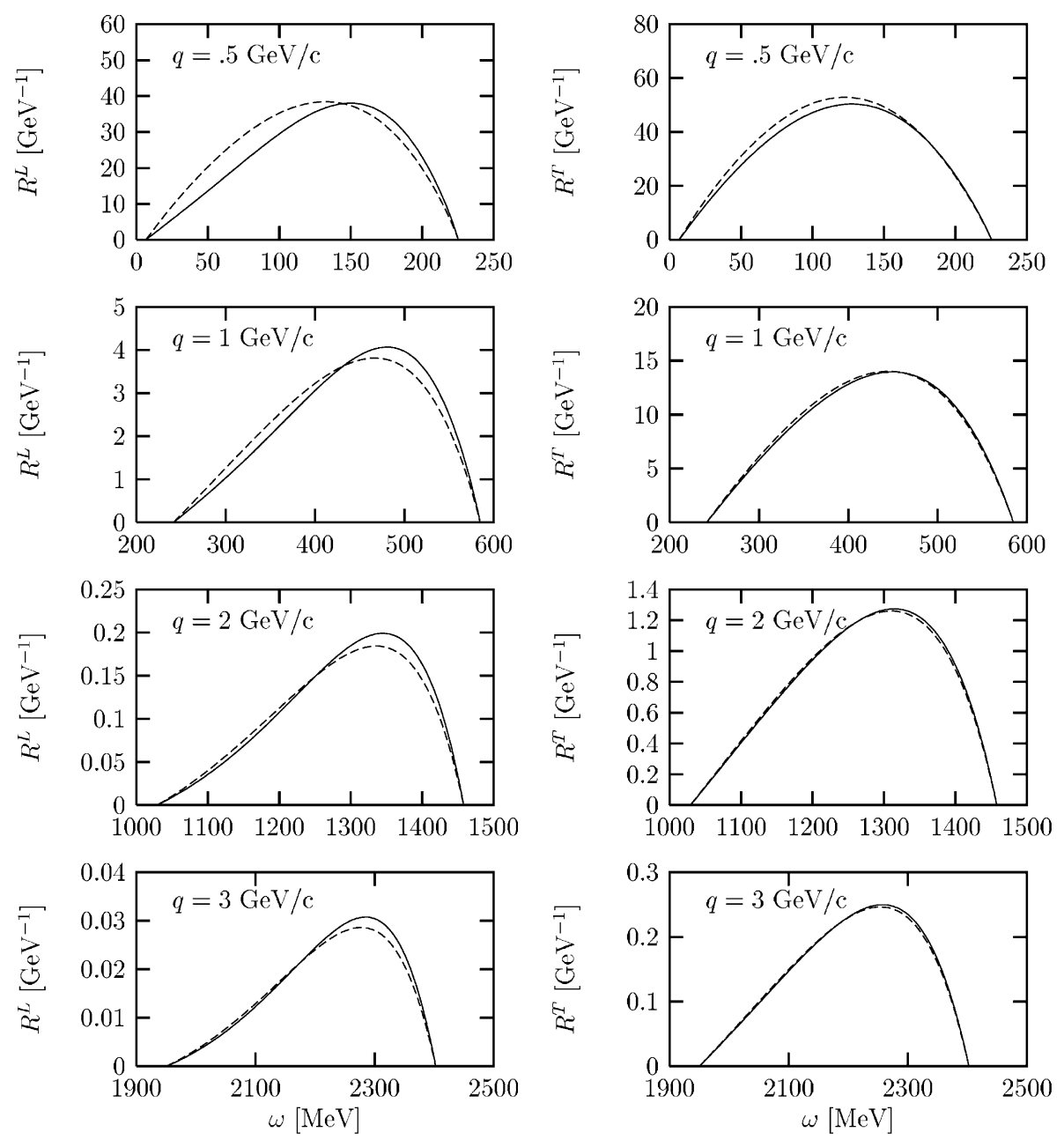

Fig. 9. Same as Fig. 7 but for the vertex correlation. Dashed: RFG responses; solid: RFG+VC contribution.

The evolution with $q$ of the $\mathrm{VC}$ in the longitudinal and transverse channels has been discussed at length in [19]. Let us summarize the basic findings. First, the VC do not saturate quite as rapidly as the MEC, although their behavior is rather similar and saturation again occurs somewhere above $q=1-1.5 \mathrm{GeV} / c$ : thus, once more, scaling of the first kind is achieved at high momentum transfers for these contributions. Moreover, similarly to the MEC case, for high $q$ the VC almost vanish around the QEP.

Finally, the vertex correlations are found to grow with $k_{\mathrm{F}}$, much as the MEC do. From a semi-relativistic point of view, we find a behavior that goes as $k_{\mathrm{F}}^{2}$. The basic conclusion is similar to that made above for the seagull contribution and hence for the total MEC at high $q$, namely, scaling of the second kind is badly broken by effects that go roughly as $k_{\mathrm{F}}^{3}$.

The role played by the SE contribution is displayed in Figs. 10 and 11. In Fig. 10 we show the total $\mathrm{RFG}+\mathrm{SE}$ responses (solid line) compared with the free RFG responses (dashed). 

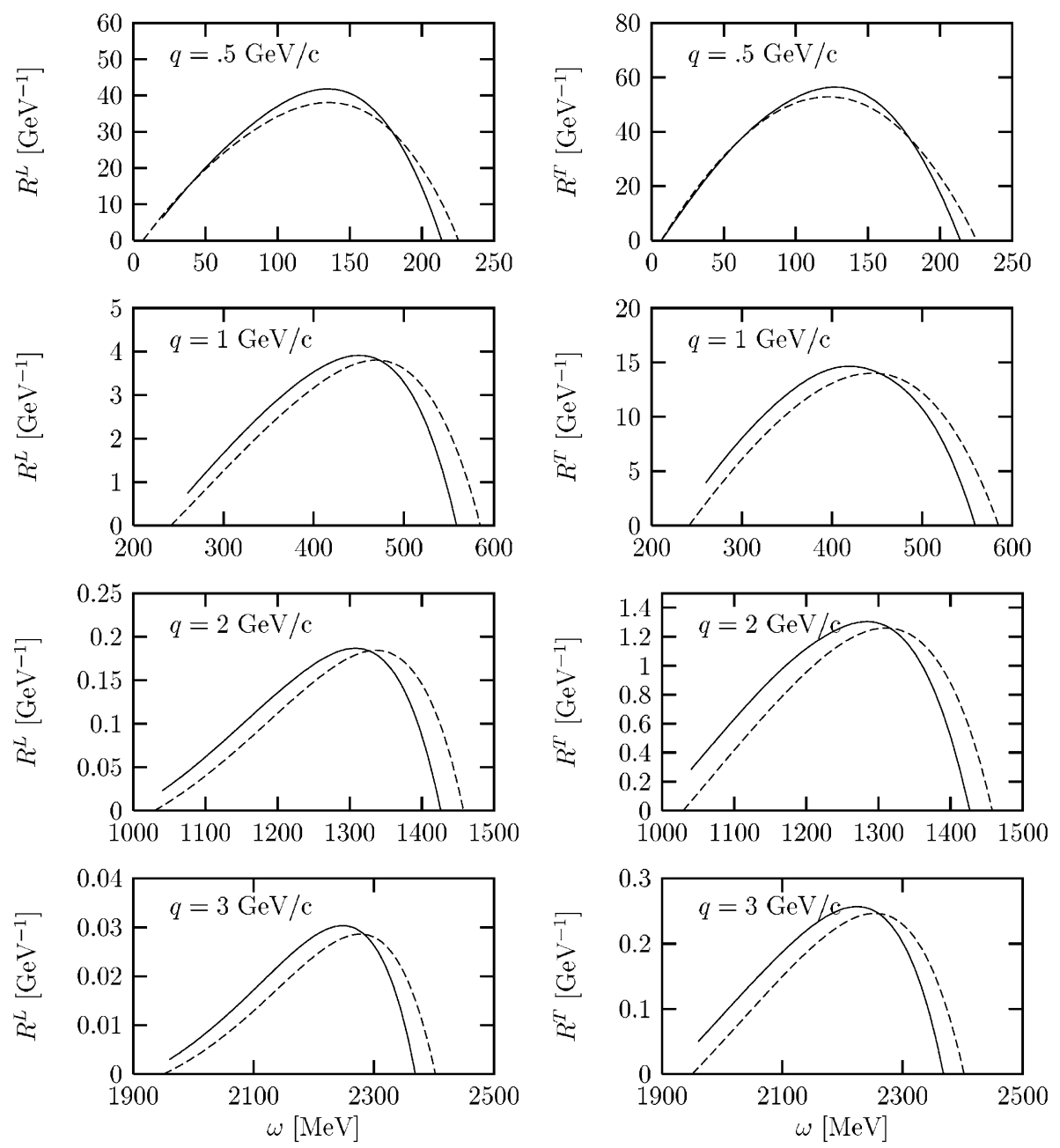

Fig. 10. Same as Fig. 7 but for the self-energy. Dashed: RFG responses; solid: RFG+SE contribution.

Note that, in contrast with the MEC and vertex correlations, which mostly contribute to only one channel (transverse and longitudinal, respectively), the impact of the self-energy on $R^{\mathrm{L}}$ and $R^{\mathrm{T}}$ is similar, leading in both cases to a softening of the responses for high $q$.

The separate particle (dashed) and hole (short-dashed) SE contributions to the longitudinal and transverse responses are presented in Fig. 11. Here, also the total SE contribution (solid) is displayed. We observe that the self-energy contribution results from a quite delicate cancellation between the responses having only the particle or only the hole dressed (Fig. 11). This was already pointed out in [51] within the framework of a treatment in which relativistic effects were partially incorporated and it is now confirmed within a fully relativistic context.

Whereas this cancellation is very substantial at $q=0.5 \mathrm{GeV} / c$, as the momentum transfer increases the imbalance between the two contributions grows. Indeed the response associated with the particle 

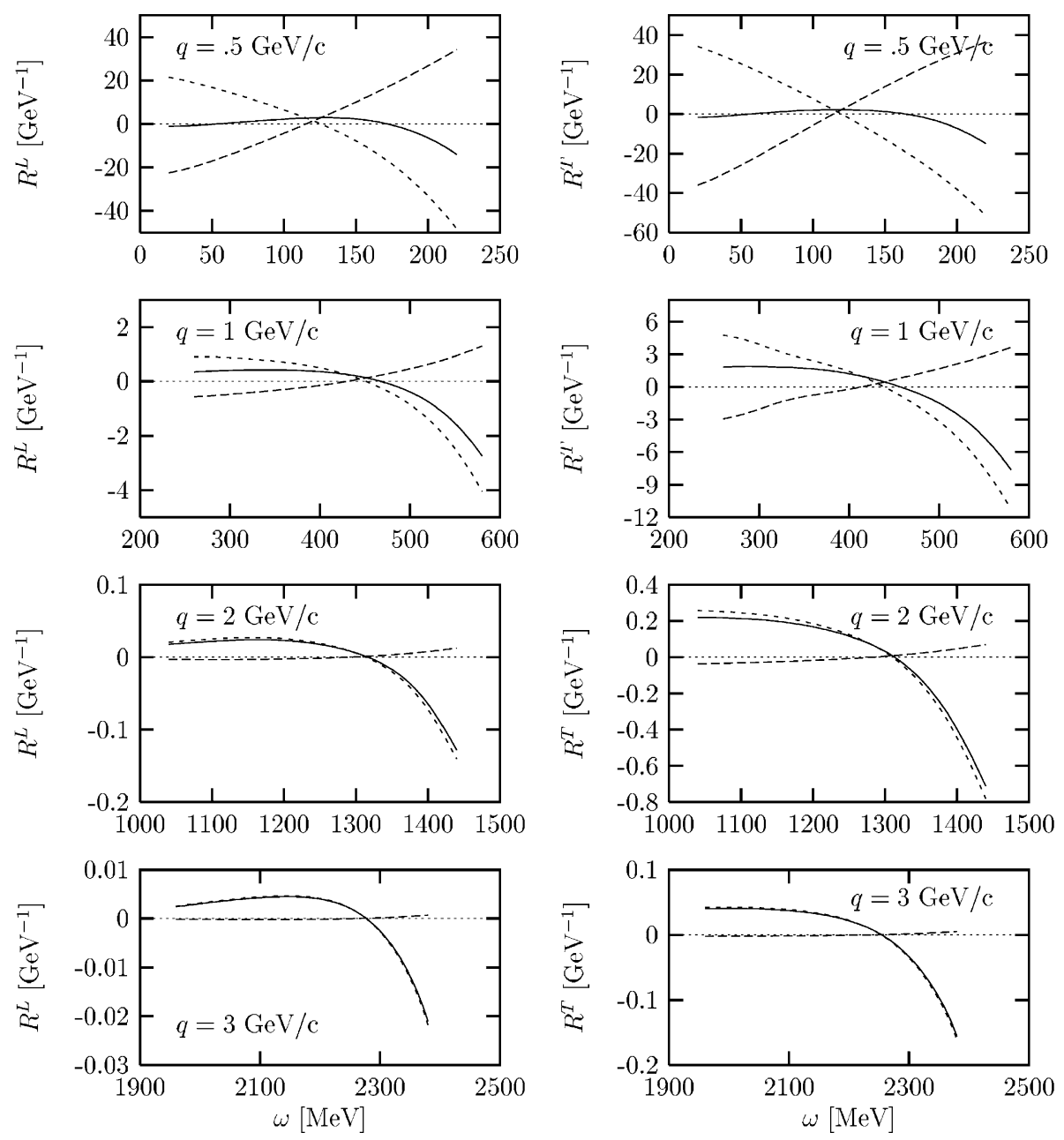

Fig. 11. Particle (dashed) and hole (short-dashed) contributions to the longitudinal (left panels) and transverse (right panels) self-energy. The solid line represents the total SE contribution.

self-energy is suppressed by the form factors and by the pion propagator, but that coming from the hole self-energy is not. As a result, for $q \geqslant 2 \mathrm{GeV} / c$ the total self-energy response is almost entirely due to the hole dressing and induces a moderate softening to the free response. Note that the SE contribution does not vanish on the borders of the response region. Moreover for high values of $\omega$ (close to the upper border) it becomes very large (Fig. 11) and yields a significant lowering of the upper $\omega$ limit in the responses. This clearly points to the insufficiency of a first-order perturbative treatment in this kinematical region, an effect already present in the partially relativized analysis of [51] and emphasized by our fully relativistic calculation. Therefore, the summation of the full Fock series becomes necessary near the upper boundary of the response.

The analysis of the scaling and superscaling properties of the self-energy correlations has been presented in [19]. In accord with the above, the particle contribution decreases with $q$, going to zero 


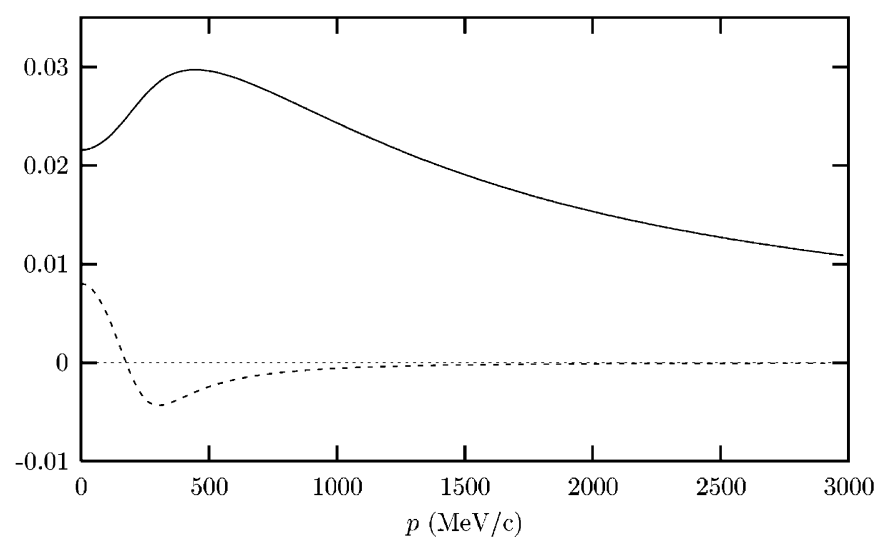

Fig. 12. The on-shell self-energy $\Sigma_{0}(p) / E_{\mathbf{p}}$ defined in Eq. (82) (solid line) and the field-strength renormalization function $\alpha(p)$ given in Eq. (100) (dashed line) plotted versus the momentum $p$.

at $q \simeq 2 \mathrm{GeV} / c$, whereas the hole contribution, although also decreasing with $q$ when not too high, saturates for $q \geqslant 1 \mathrm{GeV} / c$ (see Fig. 11). As a result the total self-energy grows with $q$ in the range $q=0.5-2 \mathrm{GeV} / c$, then stabilizes typically at about $30-40 \%$ of the free response to the left of the QEP, thus inducing an important softening of the longitudinal and transverse responses. In summary, again scaling of the first kind is achieved at momentum transfers somewhat below $2 \mathrm{GeV} / c$. Finally, we also prove that the self-energy relative contribution grows with $k_{\mathrm{F}}$, although not uniformly in the scaling variable $\psi$ (see [60-62]) - recall that in the first-order analysis presented in this paper the edges of the response region are not treated adequately for the self-energy contribution and thus should not be taken too seriously. Where the self-energy contribution is correctly modeled (away from the edges) we again see breaking of second-kind scaling by roughly $k_{\mathrm{F}}^{3}$.

In what follows we explore the impact on the responses of the new currents $j_{\mathrm{RSE} 1}^{\mu}$ and $j_{\mathrm{RSE} 2}^{\mu}$ that arise from the enhancement of the lower components of the spinors and from the field strength renormalization $\sqrt{Z_{2}(\mathbf{p})}$, respectively. In Fig. 12 we show the on-shell self-energy (solid curve) and the field strength renormalization function $\alpha(p)$ (dashed curve) given by Eqs. (82) and (100), respectively. The explicit expressions for $\Sigma_{0}(p)$ and $\alpha(p)$ are derived in Appendix E. The $\Sigma_{0}(p)$ obtained here is in good agreement with the results of [63] and its effect on the single-particle energy in Eq. (84) and on the effective mass in Eq. (86) is very small (less than $\sim 3 \%$ ). Note that $\alpha$, which is linked to the current $j_{\mathrm{RSE} 2}^{\mu}$ of Eq. (106), is much smaller than $\Sigma_{0}(p) / E_{\mathbf{p}}$, which enters in $j_{\mathrm{RSE} 1}^{\mu}$ through Eq. (105). Thus the effect of the enhancement of the lower components of the spinors dominates over the field-strength renormalization. This is very clearly seen in Fig. 13, where the various contributions to the longitudinal and transverse responses stemming from $j_{\mathrm{RSE} 1}^{\mu}$ and $j_{\mathrm{RSE} 2}^{\mu}$ are displayed versus the transferred energy $\omega$ for momentum transfer $q=0.5,1,2$ and $3 \mathrm{GeV} / c$. It is evident that the effect of $j_{\mathrm{RSE} 2}^{\mu}$ is negligible with respect to that of $j_{\mathrm{RSE} 1}^{\mu}$. The separate contributions of the particle and hole self-energies are also shown: as $q$ increases the contribution of the particle is suppressed, whereas that of the hole survives.

In Fig. 14 we compare the contribution to the longitudinal and transverse responses due to renormalization of the wave functions (solid) with that arising from renormalization of the energies 

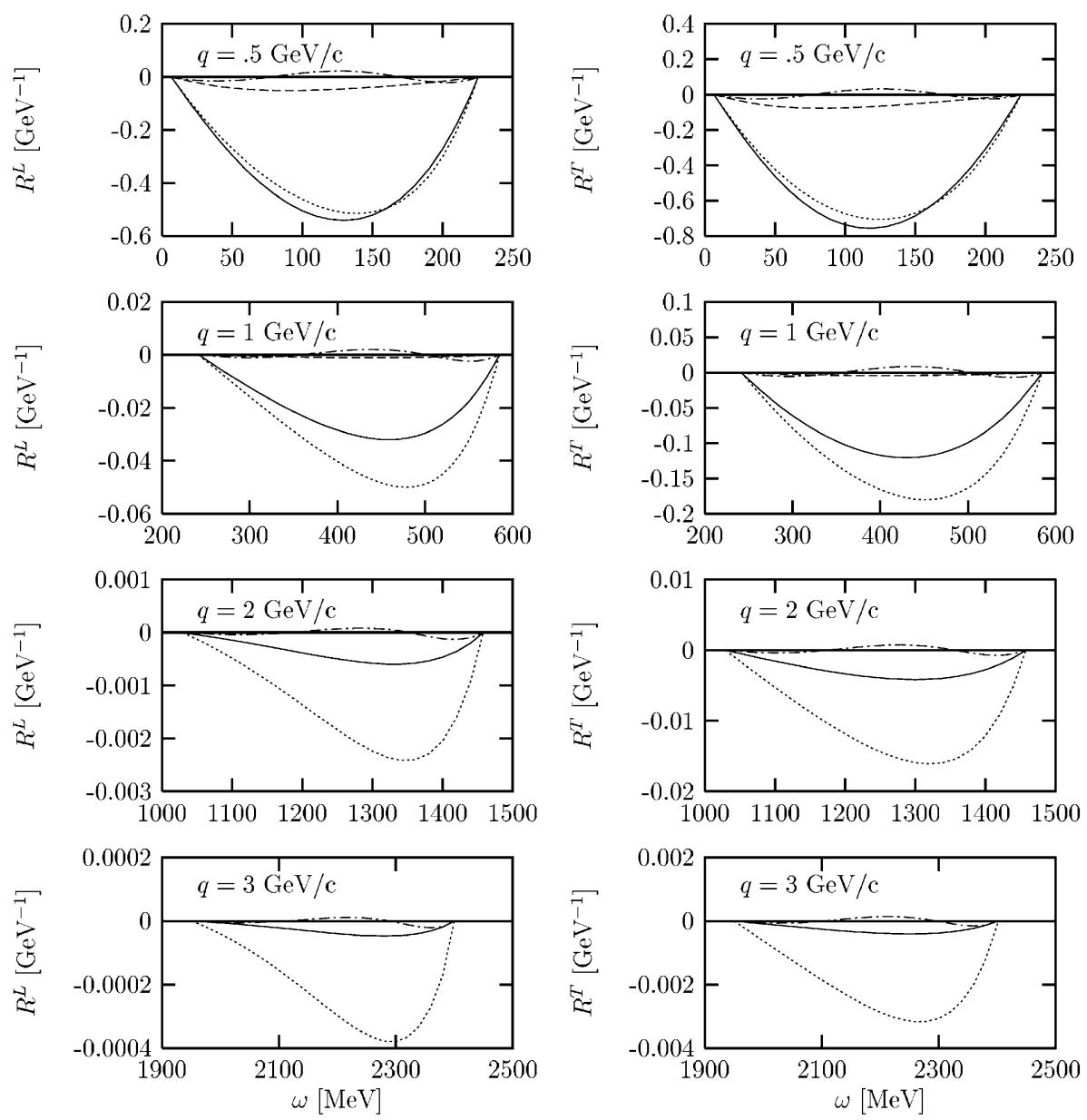

Fig. 13. The contribution of the renormalized self-energy current to the longitudinal (left panels) and transverse (right panels) responses plotted versus $\omega$. The separate contributions of the current $j_{\mathrm{RSE} 1}^{\mu}$ for the particle (solid) and hole (dotted) and of the current $j_{\mathrm{RSE2}}^{\mu}$ for the particle (dashed) and hole (dot-dashed) are displayed.

(dashed). The effect linked to modification of the energy due to the medium is the dominant one, the other being very small, especially for large values of $q$.

To complete this section we display in Fig. 15 the separate contributions of seagull (dashed), pion-in-flight (dot-dashed), VC (dotted) and SE (solid) to the longitudinal and transverse responses. Worth pointing out is the oscillatory behavior versus $\omega$ of the vertex correlations, which induces a hardening of the responses. In addition, the seagull and vertex correlations tend to cancel in the transverse channel, especially for low values of $q$, whereas for higher $q$ the MEC dominate. Note that in the $\mathrm{T}$ channel both the seagull and $\mathrm{VC}$ exactly vanish at the same value of $\omega$, the latter coinciding with the QEP for high momentum transfers, as said above. It is also important to point out that the net effect introduced by the SE contribution is in general the largest one for transfer momentum values $q \geqslant 1 \mathrm{GeV} / c$. Within the L channel, the pionic correlations (VC and SE) clearly 

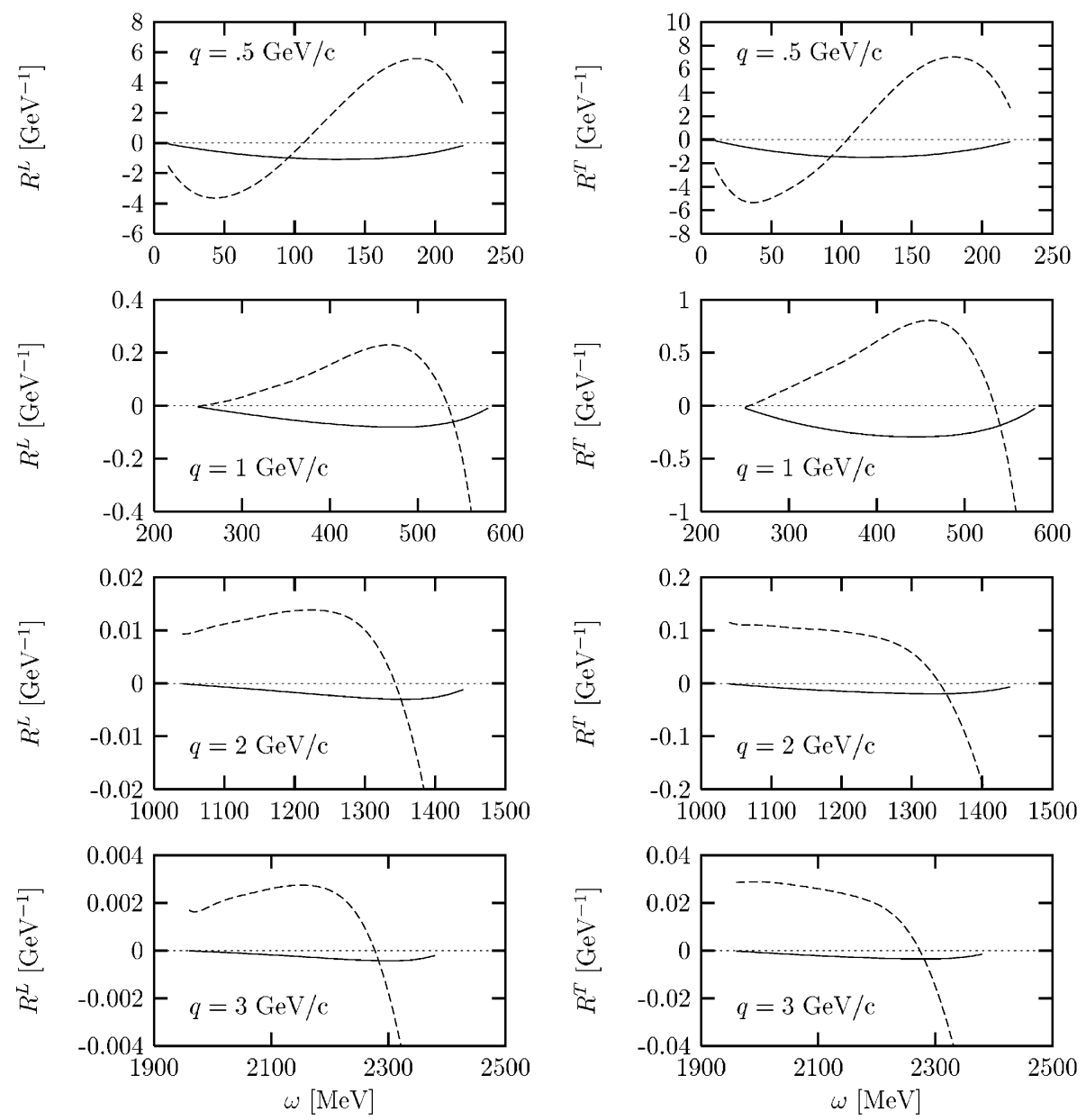

Fig. 14. The contributions of the first (solid) and second (dashed) term in Eq. (143) to the longitudinal (left panels) and transverse (right panels) responses.

dominate over the MEC. In the transverse channel, apart from the SE contribution which seems to dominate for high $q$, the seagull term is clearly more important than the $\mathrm{VC}$ one, whereas the pion-in-flight only enters for $q$ not very high.

Up to now, we have considered a pseudovector coupling for the pion, Eq. (12). We now shortly investigate the effects on the responses of using a pseudoscalar pion-nucleon Hamiltonian

$$
\mathscr{H}_{\pi N N}^{(\mathrm{PS})}=\mathrm{i} g \bar{\psi} \gamma_{5} \phi_{a} \tau_{a} \psi
$$

instead of the pseudovector one. For on-shell nucleons the Hamiltonians in Eqs. (12) and (146) are equivalent provided $f / m_{\pi}=g /(2 m)$, but for off-shell nucleons this is not so. Among the diagrams considered in our approach the only one involving off-shell nucleons is the one associated with the vertex correlations (Fig. 6e and f). Hence in Fig. 16 we compare the VC contribution to $R^{\mathrm{L}}$ and $R^{\mathrm{T}}$ 

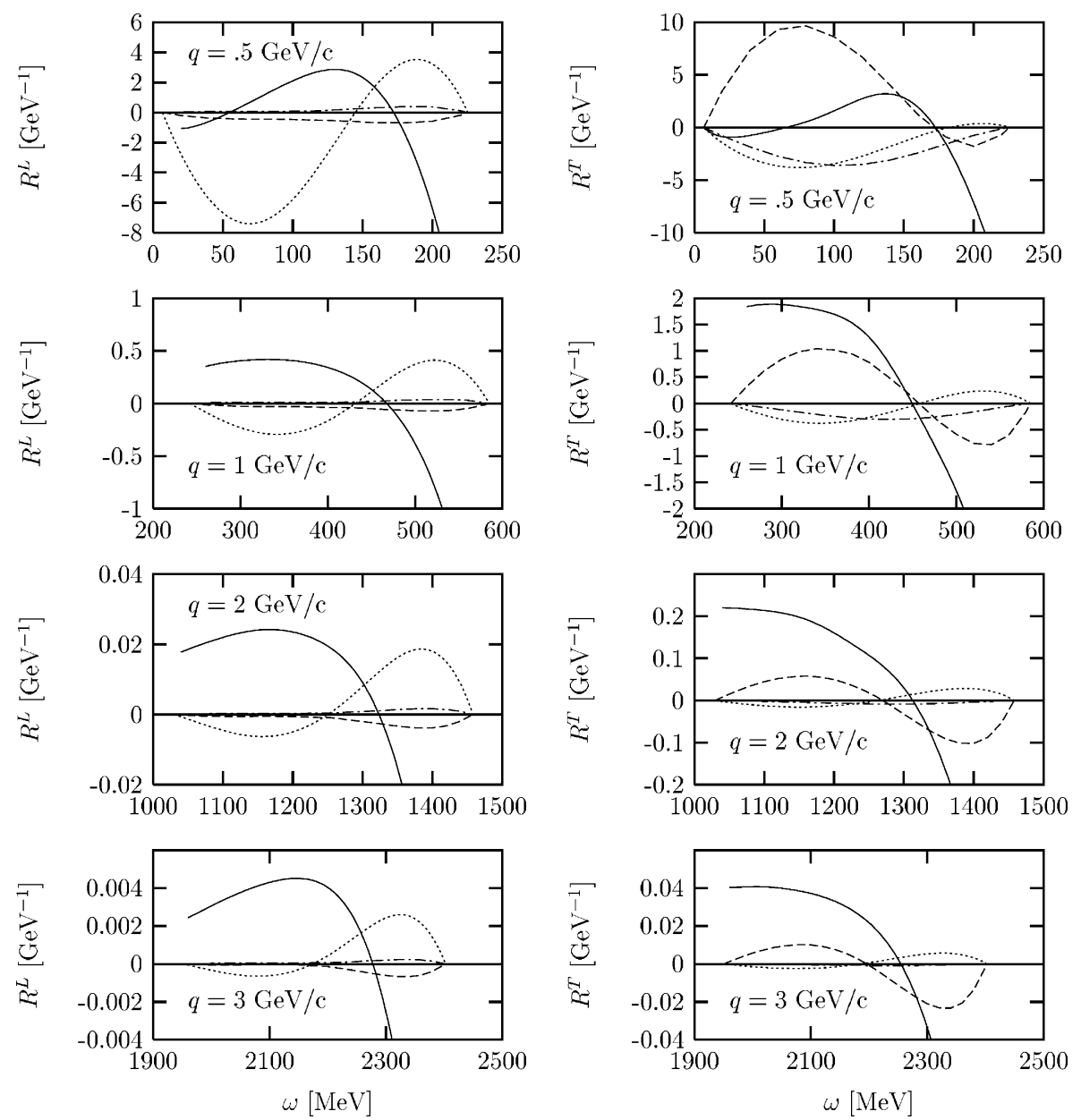

Fig. 15. Separate pion-in-flight (dot-dashed), seagull (dashed), vertex correlation (dotted) and self-energy (solid) contributions to the longitudinal (left panels) and transverse (right panels) responses.

obtained with the pseudovector (solid) and pseudoscalar (dashed) couplings. The difference between the two is especially sizable in the transverse channel (where the impact of VC is smaller) and increases with the momentum transfer.

In conclusion, in Fig. 17 we display the total responses in first order of perturbation theory and compare them with the zeroth-order ones (free responses) for several momentum transfers. Here one assesses the impact of the global two-body current contribution to the responses. First the overall effect of the two-body currents appears sufficiently modest to justify our first-order treatment. Next the softening at large $q$ appears to be common to both $\mathrm{L}$ and $\mathrm{T}$ channels, whereas at low $q$ the longitudinal response displays a hardening that is absent in the transverse one. Also evident is the already-noted nearly vanishing of the two-body correlation contribution at the peak of the free responses. Finally the unrealistic dominance of the self-energy contribution on the upper border is 

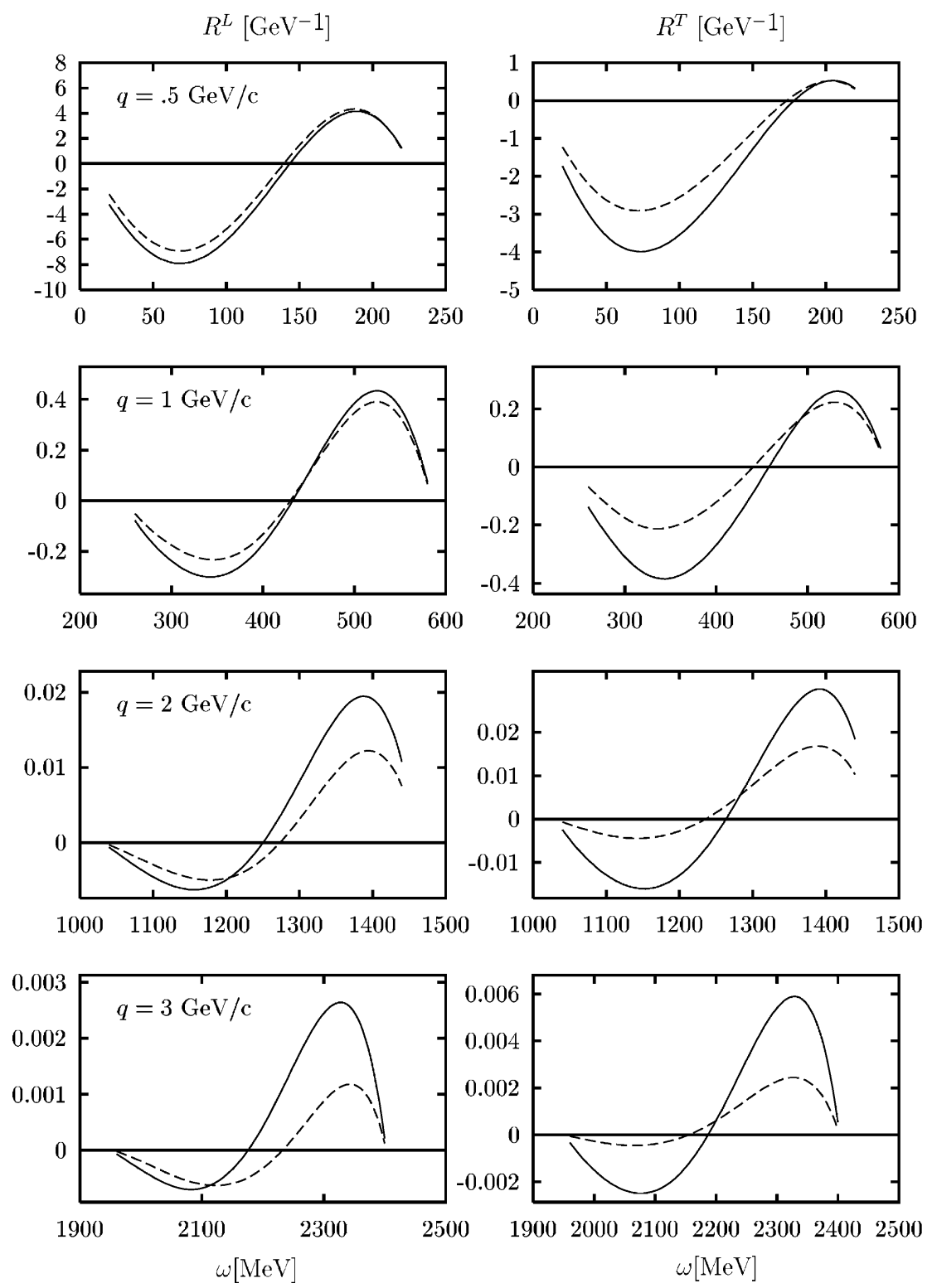

Fig. 16. Longitudinal and transverse vertex correlation responses versus $\omega$ in the pseudovector (solid) and pseudoscalar (dashed) $\pi-N$ coupling.

apparent. Summarizing, the impacts of the different first-order contributions-MEC, vertex correlations and self-energy - to the total responses are all comparable in size in the transverse channel (in the longitudinal one the MEC are negligible), their relative contribution ranging from $\sim 5 \%$ to $\sim 15 \%$ depending upon the kinematics and the Fermi momentum. 

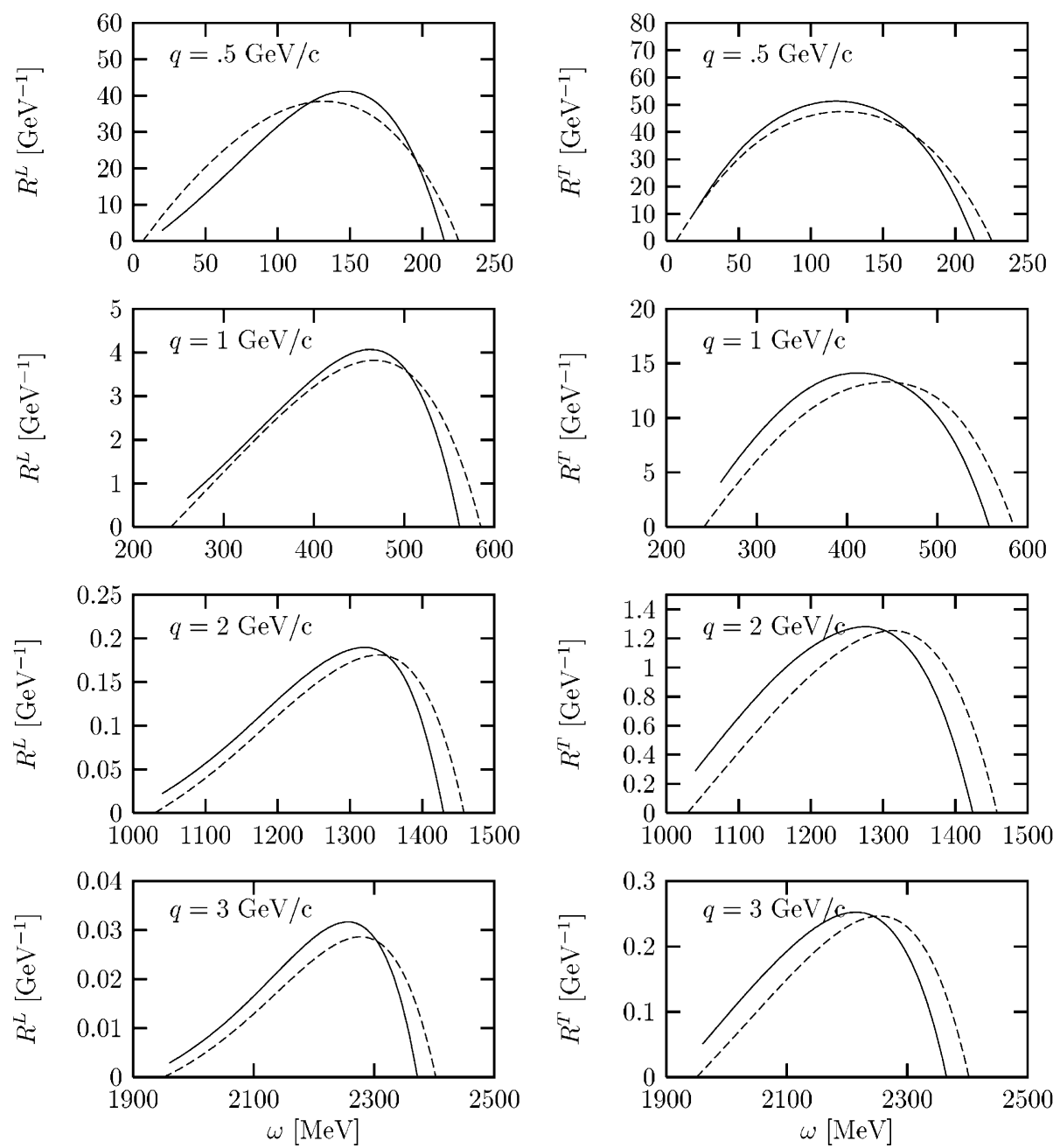

Fig. 17. Longitudinal and transverse responses versus $\omega$ including all first-order contributions (solid) compared with the free result (dashed).

\section{Parity-violating electron scattering}

In this section we deal with the parity-violating (PV) effects arising from the weak interaction between the electron and the nucleus. Such effects, which are negligible in unpolarized electron processes, can be brought to evidence by measuring the asymmetry associated with longitudinally polarized electrons having opposite helicities, namely

$$
\mathscr{A}=\frac{\mathrm{d} \sigma^{+}-d \sigma^{-}}{\mathrm{d} \sigma^{+}+d \sigma^{-}} .
$$


In this case the purely electromagnetic cross sections cancel out and one is left with the interference between the electromagnetic and neutral weak currents, corresponding to the exchange of a photon and a $Z^{0}$, respectively.

An important motivation of PV experiments (see, for example, [64] for a general review and [35] for the foundations of PV quasielastic scattering) is the measurement of the single-nucleon form factors, in particular the strange and axial ones: for this reason most experiments are presently being carried out on light nuclei, where the uncertainties associated with the nuclear model are minimized. Other motivations exist for such studies: specifically, as discussed in the following, the PV response functions display a different sensitivity to nuclear correlations compared with the parity-conserving ones: hence they could not only shed light on the part of the problem concerned with nucleon (and meson) structure, but also are being used as a test of nuclear models. In the present work we provide no details for the underlying formalism used in PV electron scattering-those discussions can be found in [64]. Our focus here is rather to place in context the expectations for PV electron scattering of what role the modeling discussed above plays.

\subsection{General formalism}

The cross section for scattering of a polarized electron with helicity $h$ reads

$$
\frac{\mathrm{d} \sigma^{(h)}}{\mathrm{d} \Omega_{\mathrm{e}}^{\prime} \mathrm{d} \omega}=\frac{\varepsilon^{\prime}}{\varepsilon}\left(\frac{2 \alpha^{2}}{Q^{4}} \eta_{\mu \nu} W^{\mu \nu}+\frac{\alpha G}{2 \sqrt{2} \pi Q^{2}} \tilde{\eta}_{\mu \nu} \tilde{W}^{\mu \nu}\right) .
$$

In Eq. (148) $G$ is the Fermi constant, $\eta_{\mu \nu}, W^{\mu \nu}$ are the leptonic and hadronic electromagnetic tensors defined in Section 2.1 and $\tilde{\eta}_{\mu \nu}, \tilde{W}^{\mu \nu}$ are the tensors arising from the $\gamma-Z^{0}$ interference. Here terms containing two weak currents have been neglected. The interference tensors read

$$
\tilde{\eta}_{\mu v}=\left(a_{\mathrm{V}}-h a_{\mathrm{A}}\right)\left(K_{\mu} K_{v}^{\prime}+K_{\mu}^{\prime} K_{v}-g_{\mu v} K \cdot K^{\prime}\right)+\left(a_{\mathrm{A}}-h a_{\mathrm{V}}\right) \mathrm{i} \epsilon_{\mu \nu \rho \sigma} K^{\rho} K^{\prime \sigma}
$$

with $a_{\mathrm{A}}=-1$ and $a_{\mathrm{V}}=4 \sin ^{2} \theta_{\mathrm{W}}-1, \theta_{\mathrm{W}}$ being the weak mixing angle, and

$$
\tilde{W}^{\mu v}=\overline{\sum_{i}} \sum_{f}\left\langle f\left|\hat{J}_{\mathrm{em}}^{\mu}(Q)\right| i\right\rangle^{*}\left\langle f\left|\hat{J}_{\mathrm{wn}}^{v}(Q)\right| i\right\rangle \delta\left(E_{i}+\omega-E_{f}\right),
$$

$\hat{J}_{\text {em }}^{\mu}(Q)$ and $\hat{J}_{\text {wn }}^{v}(Q)$ being the nuclear electromagnetic and weak neutral currents, respectively.

When the difference of cross sections corresponding to opposite electron helicities is taken, the electromagnetic term in Eq. (148) cancels out and the resulting PV expression reads

$$
\begin{aligned}
\left(\frac{\mathrm{d} \sigma}{\mathrm{d} \Omega_{\mathrm{e}}^{\prime} \mathrm{d} \omega}\right)_{\mathrm{PV}} & \equiv \frac{1}{2}\left(\frac{\mathrm{d} \sigma^{(+)}}{\mathrm{d} \Omega_{\mathrm{e}}^{\prime} \mathrm{d} \omega}-\frac{\mathrm{d} \sigma^{(-)}}{\mathrm{d} \Omega_{\mathrm{e}}^{\prime} \mathrm{d} \omega}\right) \\
& =\mathscr{A}_{0} \sigma_{\mathrm{M}}\left[v_{\mathrm{L}} R_{\mathrm{AV}}^{\mathrm{L}}(q, \omega)+v_{\mathrm{T}} R_{\mathrm{AV}}^{\mathrm{T}}(q, \omega)+v_{\mathrm{T}} R_{\mathrm{VA}}^{\mathrm{T}^{\prime}}(q, \omega)\right],
\end{aligned}
$$

where

$$
\mathscr{A}_{0}=\frac{G\left|Q^{2}\right|}{2 \sqrt{2} \pi \alpha},
$$


$\sigma_{\mathrm{M}}$ is the Mott cross section in Eq. (4), the leptonic kinematical factors $v_{\mathrm{L}}$ and $v_{\mathrm{T}}$ are given by Eqs. (6) and (7) and

$$
v_{\mathrm{T}^{\prime}}=\tan \frac{\theta_{\mathrm{e}}}{2} \sqrt{-\left(\frac{Q^{2}}{q^{2}}\right)+\tan ^{2} \frac{\theta_{\mathrm{e}}}{2}} .
$$

In terms of nuclear response functions the asymmetry in Eq. (147) reads

$$
\mathscr{A}=\mathscr{A}_{0} \frac{v_{\mathrm{L}} R_{\mathrm{AV}}^{\mathrm{L}}+v_{\mathrm{T}} R_{\mathrm{AV}}^{\mathrm{T}}+v_{\mathrm{T}^{\prime}} R_{\mathrm{VA}}^{\mathrm{T}^{\prime}}}{v_{\mathrm{L}} R^{\mathrm{L}}+v_{\mathrm{T}} R^{\mathrm{T}}} .
$$

\subsection{PV response functions}

The PV response functions appearing in Eq. (151) are linked to the interference hadronic tensor in Eq. (150) by the following relations:

$$
\begin{aligned}
& R_{\mathrm{AV}}^{\mathrm{L}}(q, \omega)=a_{\mathrm{A}}\left(\frac{q^{2}}{Q^{2}}\right)^{2}\left[\tilde{W}^{00}-\frac{\omega}{q}\left(\tilde{W}^{03}+\tilde{W}^{30}\right)+\frac{\omega^{2}}{q^{2}} \tilde{W}^{33}\right], \\
& R_{\mathrm{AV}}^{\mathrm{T}}(q, \omega)=a_{\mathrm{A}}\left(\tilde{W}^{11}+\tilde{W}^{22}\right), \\
& R_{\mathrm{VA}}^{\mathrm{T}^{\prime}}(q, \omega)=-\mathrm{i} a_{\mathrm{V}}\left(\tilde{W}^{12}-\tilde{W}^{21}\right) .
\end{aligned}
$$

The subscript AV in the PV responses denotes interferences of axial-vector leptonic currents with vector hadronic currents, and the reverse for the subscript VA.

Within the context of the RFG model the interference hadronic tensor is

$$
\tilde{W}^{\mu \nu}=\frac{3 Z}{8 \pi k_{\mathrm{F}}^{3} q} \int_{h_{0}}^{k_{\mathrm{F}}} h \mathrm{~d} h\left(\omega+E_{\mathbf{h}}\right) \int_{0}^{2 \pi} \mathrm{d} \phi_{h} \sum_{s_{p}, s_{h}} \frac{m^{2}}{E_{\mathbf{p}} E_{\mathbf{h}}} 2 \operatorname{Re}\left[j_{\mathrm{em}}^{\mu}(\mathbf{p}, \mathbf{h})^{*} j_{\mathrm{wn}}^{v}(\mathbf{p}, \mathbf{h})\right],
$$

where the electromagnetic current $j_{\mathrm{em}}^{\mu}$ includes both the single-nucleon one-body and the two-body (MEC and correlation) currents discussed in the previous section, i.e., $j_{\mathrm{em}}^{\mu}=j_{\mathrm{OB}}^{\mu}+j_{\mathrm{MEC}}^{\mu}+j_{\mathrm{cor}}^{\mu}$. In this work we include in the weak neutral current only the one-body contribution (see Fig. 18), namely

$$
j_{\text {wn }}^{v}(\mathbf{p}, \mathbf{h})=\bar{u}(\mathbf{p})\left(\tilde{F}_{1} \gamma^{\nu}+\mathrm{i} \frac{\tilde{F}_{2}}{2 m} \sigma^{\nu \rho} Q_{\rho}+\tilde{G}_{\mathrm{A}} \gamma_{5} \gamma^{\nu}\right) u(\mathbf{h}),
$$

where the Pauli and Dirac form factors are

$$
\begin{aligned}
& \tilde{F}_{1}=\frac{\tilde{G}_{\mathrm{E}}+\tau \tilde{G}_{\mathrm{M}}}{1+\tau}, \\
& \tilde{F}_{2}=\frac{\tilde{G}_{\mathrm{E}}-\tilde{G}_{\mathrm{M}}}{1+\tau} .
\end{aligned}
$$

Thus we neglect the direct coupling of a $Z^{0}$ to the pion (important clues for the understanding of the weak-neutral sector of the MEC should be found in the study of pion electroproduction on the 


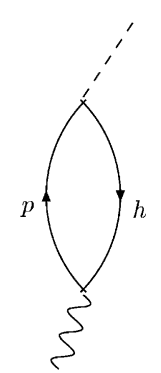

(a)

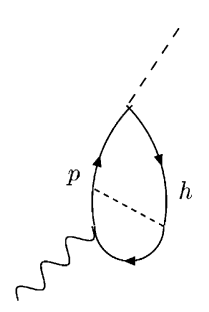

(e)

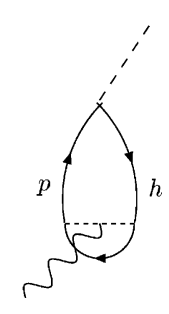

(b)

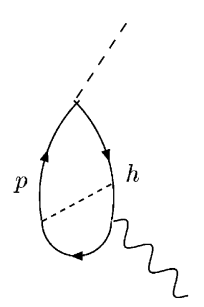

(f)

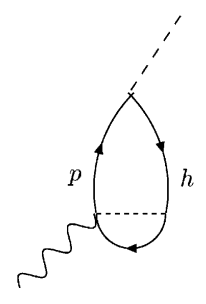

(c)

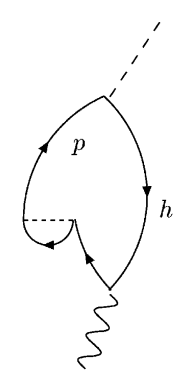

(g)

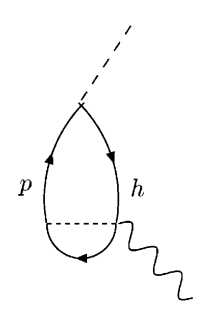

(d)

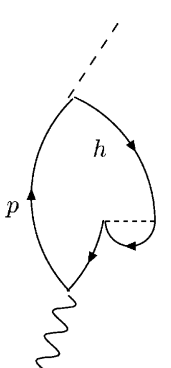

(h)

Fig. 18. Feynman diagrams of the free (a) and first-order pion-in-flight (b), seagull (c and d), vertex correlation (e and f) and self-energy (g and h) PV polarization propagator. The external wavy and dashed lines represent a photon and a $Z^{0}$ boson, respectively.

nucleon, where a $Z^{0}$ is exchanged with the nucleon. This topic has recently been investigated in [65].)

Within the standard model at tree level, the weak neutral form factors are linked to the electromagnetic ones by the following relations (possible contributions from the strange quark are neglectedthese can be included in a straightforward way $[35,64]$ and do not provide the primary focus of the present discussions):

$$
\begin{aligned}
& \tilde{G}_{\mathrm{Ep}(\mathrm{n})}=\beta_{\mathrm{V}}^{\mathrm{p}} G_{\mathrm{Ep}(\mathrm{n})}+\beta_{\mathrm{V}}^{\mathrm{n}} G_{\mathrm{En}(\mathrm{p})}, \\
& \tilde{G}_{\mathrm{Mp}(\mathrm{n})}=\beta_{\mathrm{V}}^{\mathrm{p}} G_{\mathrm{Mp}(\mathrm{n})}+\beta_{\mathrm{V}}^{\mathrm{n}} G_{\mathrm{Mn}(\mathrm{p})}, \\
& \tilde{G}_{\mathrm{Ap}(\mathrm{n})}=\beta_{\mathrm{A}}^{\mathrm{p}} G_{\mathrm{Ap}(\mathrm{n})}+\beta_{\mathrm{A}}^{\mathrm{n}} G_{\mathrm{An}(\mathrm{p})},
\end{aligned}
$$

where

$$
\beta_{\mathrm{V}}^{\mathrm{p}}=\frac{1}{2}\left(1-4 \sin ^{2} \theta_{\mathrm{W}}\right), \quad \beta_{\mathrm{V}}^{\mathrm{n}}=\beta_{\mathrm{A}}^{\mathrm{n}}=-\beta_{\mathrm{A}}^{\mathrm{p}}=\frac{1}{2} .
$$

The one-body contribution to the three PV responses can be evaluated analytically in RFG, yielding (see, for example [35])

$$
\begin{aligned}
& R_{\mathrm{AV}}^{\mathrm{L}, \mathrm{T}}(q, \omega)=a_{\mathrm{A}} R_{0}(q, \omega)\left[\tilde{U}_{\mathrm{p}}^{\mathrm{L}, \mathrm{T}}(q, \omega)+\tilde{U}_{\mathrm{n}}^{\mathrm{L}, \mathrm{T}}(q, \omega)\right], \\
& R_{\mathrm{VA}}^{\mathrm{T}^{\prime}}(q, \omega)=a_{\mathrm{V}} R_{0}(q, \omega)\left[\tilde{U}_{\mathrm{p}}^{\mathrm{T}^{\prime}}(q, \omega)+\tilde{U}_{\mathrm{n}}^{\mathrm{T}^{\prime}}(q, \omega)\right],
\end{aligned}
$$


where $R_{0}$ has been defined in Eq. (128) and

$$
\begin{aligned}
& \tilde{U}_{\mathrm{p}(\mathrm{n})}^{\mathrm{L}}(q, \omega)=\frac{\kappa^{2}}{\tau}\left\{G_{\mathrm{Ep}(\mathrm{n})} \tilde{G}_{\mathrm{Ep}(\mathrm{n})}+\frac{\Delta}{1+\tau}\left[G_{\mathrm{Ep}(\mathrm{n})} \tilde{G}_{\mathrm{Ep}(\mathrm{n})}+\tau G_{\mathrm{Mp}(\mathrm{n})} \tilde{G}_{\mathrm{Mp}(\mathrm{n})}\right]\right\}, \\
& \tilde{U}_{\mathrm{p}(\mathrm{n})}^{\mathrm{T}}(q, \omega)=2 \tau G_{\mathrm{Mp}(\mathrm{n})} \tilde{G}_{\mathrm{Mp}(\mathrm{n})}+\frac{\Delta}{1+\tau}\left[G_{\mathrm{Ep}(\mathrm{n})} \tilde{G}_{\mathrm{Ep}(\mathrm{n})}+\tau G_{\mathrm{Mp}(\mathrm{n})} \tilde{G}_{\mathrm{Mp}(\mathrm{n})}\right], \\
& \tilde{U}_{\mathrm{p}(\mathrm{n})}^{\mathrm{T}^{\prime}}(q, \omega)=2 \sqrt{\tau(1+\tau)} G_{\mathrm{Mp}(\mathrm{n})} \tilde{G}_{\mathrm{Ap}(\mathrm{n})}(1+\tilde{\Delta}),
\end{aligned}
$$

with $\Delta$ given by Eq. (133) and

$$
\tilde{\Delta} \equiv \frac{1}{\kappa} \sqrt{\frac{\tau}{1+\tau}}\left[\frac{1}{2}\left(\varepsilon_{\mathrm{F}}+\varepsilon_{0}\right)+\lambda\right]-1 .
$$

The two-body contributions involve instead multidimensional integrals, to be numerically evaluated.

\subsection{Results}

In this section we analyze the PV response functions labeled $\mathrm{L}, \mathrm{T}$ and $\mathrm{T}^{\prime}$ and the associated asymmetry for various values of the momentum transfer. In [66] results for the PV responses in a relativized continuum shell model were presented in the impulse approximation for finite, closed shell nuclei. Two-body currents were not included in that calculation. In [51,55], a semi-relativistic analysis of the PV responses has been presented, showing the dominance of pionic correlations in the longitudinal channel. Here we perform a fully relativistic calculation, which confirms the above findings, and extends them to higher values of the momentum transfer.

In Fig. 19 we display the PV responses for four values of the momentum transfer $q$. The dashed line corresponds to the free RFG, the dotted line includes MEC and vertex correlations, whereas the solid line also includes the self-energy contribution.

One observes that in the $\mathrm{T}$ and $\mathrm{T}^{\prime}$ channels (central and right columns) the main effect arises from the self-energy, which tends to soften the response function, similar to what happens in the parity-conserving case, whereas the MEC and VC effect is very tiny. Note also that the axial response is proportional to the transverse one, the factor between the two being roughly $\sqrt{1+1 / \tau} a_{\mathrm{V}} G_{\mathrm{A}}^{(1)} / G_{\mathrm{M}}^{(1)}$ : this agrees with the conjecture of $[41,55]$, which is proven here to be valid within a fully relativistic context. It also appears that the self-energy contribution increases in going from $q=500 \mathrm{MeV} / c$ to $1 \mathrm{GeV} / c$, then saturates for higher values of $q$ (thus scaling of first kind is fulfilled). This is due to the same particle-hole cancellation mechanism occurring in the electromagnetic case.

In the longitudinal channel, the self-energy gives instead a very small contribution compared with the MEC and vertex correlations. The effect of the SE contribution is found to increase with $q$, but always remains smaller than the one arising from the other correlations. Indeed the one-body longitudinal response is suppressed due to a delicate cancellation between the isoscalar and isovector responses [35]. Physically this occurrence reflects the fact that the electric form factor in one of the two vertices of Fig. 18a is always very small, for both protons and neutrons. When isospin correlations are taken into account this balance can be disrupted, as pointed out in [51,55,67], thus yielding the large effects observed in Fig. 19. Indeed in Fig. 18e and f a proton can be converted 

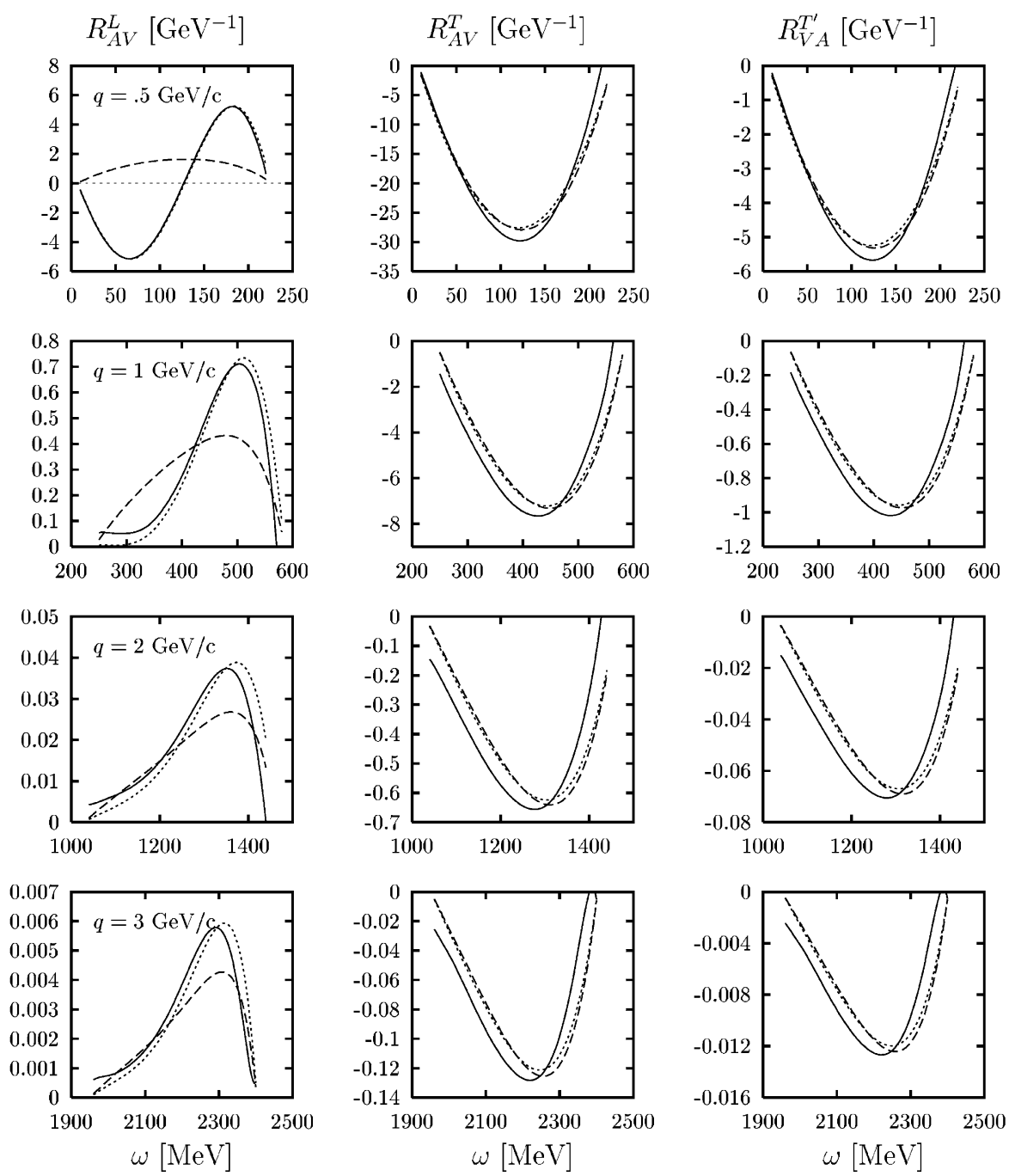

Fig. 19. The longitudinal (left panels), transverse (central panels) and axial (right panels) PV responses plotted versus $\omega$. Dashed line: one-body contribution; dotted line: one-body+MEC+VC; solid line: total (including SE).

into a neutron, leading to two large couplings, $G_{\text {Ep }}$ and $\tilde{G}_{\mathrm{En}}$. Using different language, the MEC and $\mathrm{VC}$ are more effective than the $\mathrm{SE}$ in $R_{\mathrm{AV}}^{\mathrm{L}}$, since they act differently in the two isospin channels. Indeed the $\mathrm{VC}$ carries a factor -3 in the isoscalar response and +1 in the isovector one and the MEC are purely isovector, whereas the SE has almost the same impact in the two channels.

This is clearly illustrated in Fig. 20, where the separate seagull (dashed), pion-in-flight (dotdashed) and VC (solid) contributions are displayed. In the $\mathrm{L}$ channel the role of MEC is almost negligible, in agreement with the findings for the electromagnetic $R^{\mathrm{L}}$, whereas the effect of the vertex correlations is dominant, especially at small values of $q$. In the $\mathrm{T}$ and $\mathrm{T}^{\prime}$ channels, the balance between MEC and VC is similar to that occurring for the electromagnetic $R^{\mathrm{T}}$ (see Fig. 15): the pion-in-flight gives the smallest contribution, particularly for large $q$, whereas the seagull 

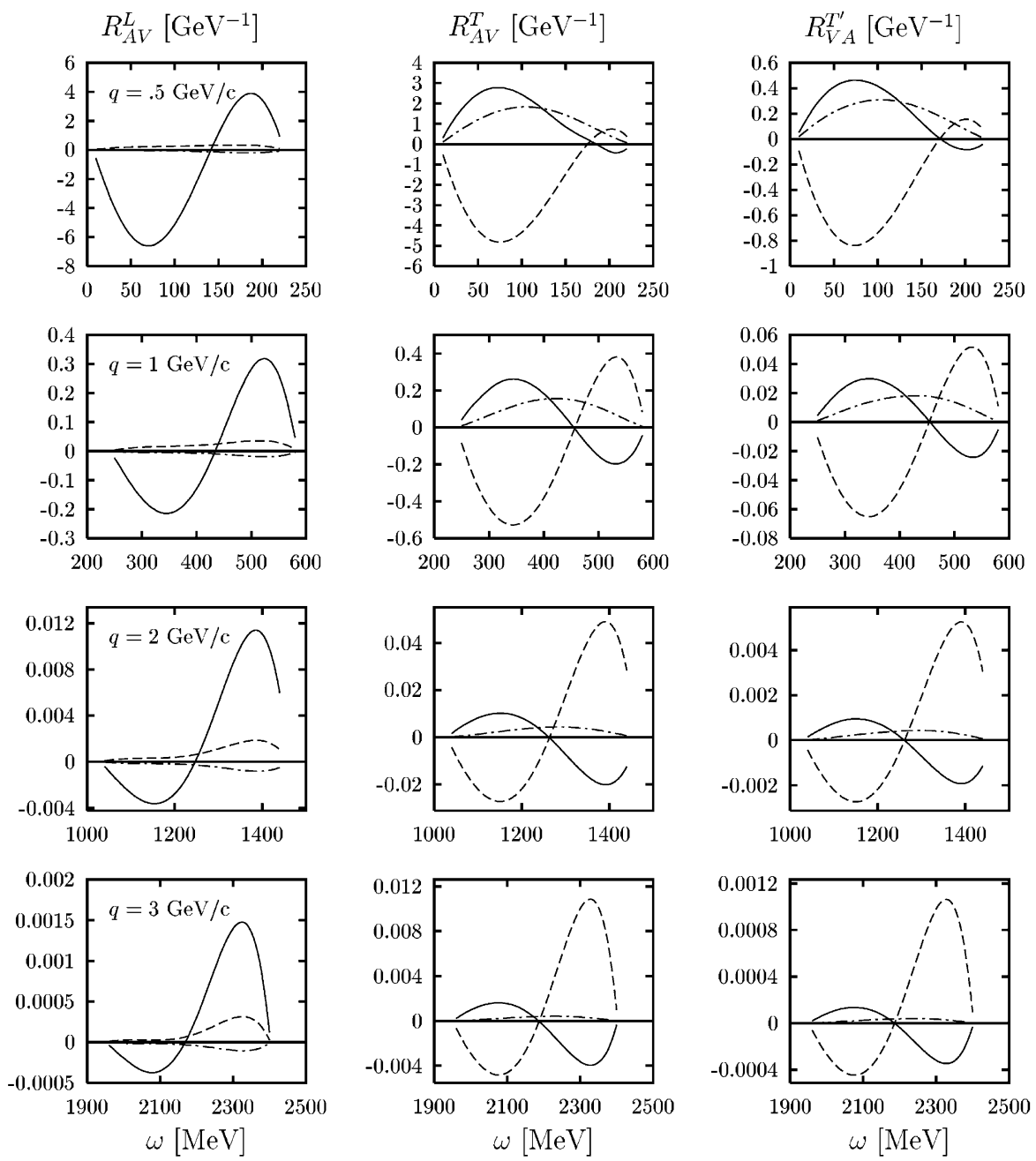

Fig. 20. Separate contributions to the PV longitudinal (left panels), transverse (central panels) and axial (right panels) responses plotted versus $\omega$. Solid: VC; dashed: seagull; dot-dashed: pion-in-flight.

dominates for all $q$ and tends to cancel the VC contribution. Note also that the seagull and VC vanish exactly at the same value of $\omega$, which, for high $q$, coincides with the QEP.

Since the three PV responses are not at present experimentally separable, we now explore the effect of the pionic physics on the asymmetry in Eq. (154). In Fig. 21 we show $\mathscr{A}$ at various values of the momentum transfer $q$ and of the electron scattering angle $\theta_{\mathrm{e}}$ for the free RFG (dashed), and including the MEC and VC (dot-dashed) or the MEC, VC and SE (solid) contributions. Clearly the pionic correlations are mostly felt at low values of $\theta_{\mathrm{e}}$ (left panel), where the longitudinal response is enhanced by the kinematical factor $v_{\mathrm{L}}$, and at low values of $q$, where the vertex correlations dominate. At high values of $\theta_{\mathrm{e}}$ (right panel) the asymmetry is totally insensitive to pions, because the effect of the SE (which gives the main contribution) cancels between the PV and PC responses appearing in the numerator and denominator of Eq. (154). 

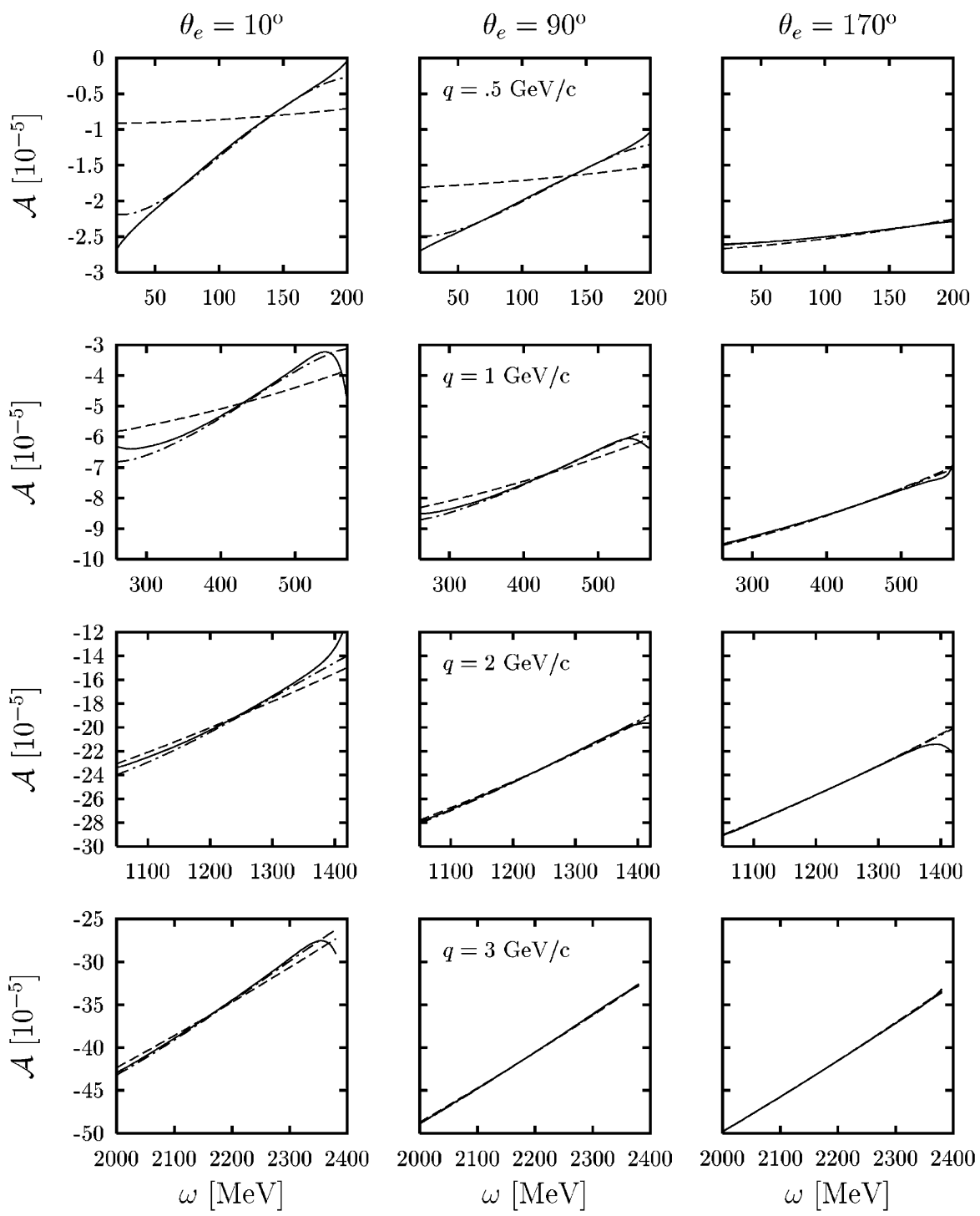

Fig. 21. The PV asymmetry displayed versus $\omega$ for various values of the momentum transfer $q$ and the scattering angle $\theta_{\mathrm{e}}$. Dashed: one-body; dot-dashed: one-body+MEC+VC; solid: total.

We thus conclude that the extraction (at large electron angles) of the axial nucleonic form factor $G_{\mathrm{A}}$ is almost independent of the nuclear model. On the contrary, at small angles PV experiments can measure the strange electric content of the nucleon only if a good control of the nuclear dynamics is achieved, since the isospin correlations give very large effects. Conversely, interesting insight into the latter can in principle be gained here. Our results show that only at very large momentum transfer does the forward-angle asymmetry become insensitive to pionic correlations and hence suitable for assessing the strangeness content of the nucleon. 


\section{Non-relativistic reductions}

For years most of the effects introduced by the two-body pionic currents in electron scattering reactions have been explored assuming different types of non-relativistic reduction [28,68-72]. Not only non-relativistic wave functions have been used, but also non-relativistic current operators derived from a direct Pauli reduction have been considered. Focusing on the pionic effects on the hadronic $\left(e, e^{\prime}\right)$ response functions one has to deal with the single-nucleon electromagnetic (electroweak in general) current and the various two-body pionic currents discussed previously. Concerning the former, an improved version of the single-nucleon electromagnetic current has been suggested in [66], where the expression of the current is derived as a non-relativistic expansion in terms of the dimensionless parameter $\eta \equiv p / m, p$ being the three-momentum of the struck nucleon. In Appendix F we review this approach - which we call semi-relativistic (SR) - and compare it with the traditional non-relativistic reduction, where the non-relativistic expansion is performed with the additional assumption $\kappa \equiv q / 2 m \ll 1$ and $\lambda \equiv \omega / 2 m \ll 1$. As shown in [20,66] and in Appendix F, the expansion of the current to first order in the variable $\eta$ yields quite simple expressions; moreover the various pieces of the relativized current differ from the traditional non-relativistic expressions only by multiplicative $(q, \omega)$-dependent factors, and therefore are easy to implement in already existing non-relativistic models.

The SR form of the OB electromagnetic current operator was first checked in [66], where the inclusive longitudinal and transverse responses of a non-relativistic Fermi gas were found to agree with the exact relativistic result within a few percent if one uses relativistic kinematics when computing the energy of the ejected nucleon. Recently the same expansion has been tested with great success by comparing with the relativistic exclusive polarized responses for the ${ }^{2} \mathrm{H}\left(e, e^{\prime} p\right)$ reaction at high momentum transfers [73]. This relativized current has also been applied to the calculation of inclusive and exclusive responses that arise in the scattering of polarized electrons from unpolarized [74] and polarized nuclei [75-78]. Finally, it also has been compared with a fully relativistic DWIA calculation of $\left(e, e^{\prime} p\right)$ observables for $|Q|^{2}=0.8(\mathrm{GeV} / c)^{2}$ in $[79,80]$. A systematic analysis of the SR approximation in the case of $\left(\vec{e}, e^{\prime} \vec{N}\right)$ reactions has been presented in [81].

Alternative expansions, in powers of the initial nucleon momentum, of the structure functions of nuclei have recently been proposed [15] and "recipes" to obtain a relativistic structure function from its non-relativistic analog by changing the scaling variable and performing an energy shift have been suggested. The so-called three-dimensional reduction model, which includes final-state interactions, has been tested in the case of a deuteron target, but not for $A \geqslant 3$.

The necessity of an SR form for the current even for moderate momentum transfer is demonstrated in Fig. 22, where we compare the traditional non-relativistic results for the electromagnetic responses with the fully relativistic calculation for a non-interacting system. It clearly appears that for low densities and momentum transfers the two approaches are equivalent, but that the two curves deviate from each other as $q$ and $k_{\mathrm{F}}$ increase. One of the effects of relativity is the shrinking of the response region [25] and is already significant at $q=400 \mathrm{MeV} / c$. This effect, which arises from the relativistic kinematics in the energy-conserving delta-function appearing in the responses, can be accounted for approximately by the replacement

$$
\lambda \rightarrow \lambda(1+\lambda)
$$



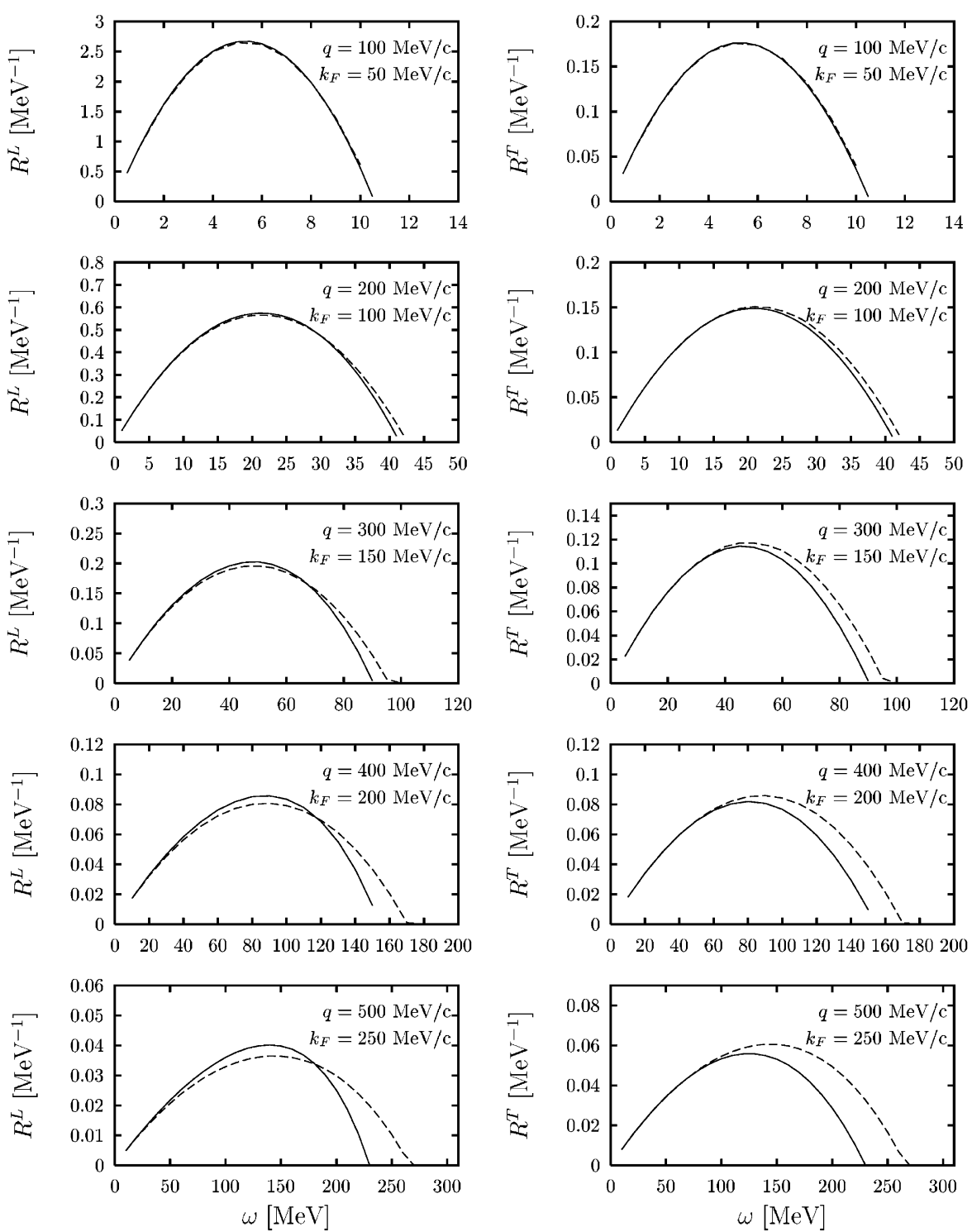

Fig. 22. The one-body longitudinal (left panels) and transverse (right panels) responses displayed versus $\omega$ for various values of the momentum transfer $q$ and of the Fermi momentum $k_{\mathrm{F}}$. Dashed: non-relativistic; solid: relativistic.

Another effect, stemming from the non-relativistic reduction of the currents, relates to the enhancement of the longitudinal response and to the reduction of the transverse one due to relativity. Such an effect can be mimicked by the kinematical factors $\kappa^{2} / \tau$ (in the $\mathrm{L}$ channel) and $\tau / \kappa^{2}$ (in the $\mathrm{T}$ channel), which naturally emerge from the $\eta$ expansion illustrated in Appendix F. When included in the non-relativistic responses these factors, together with the prescription of Eq. (172), allow one to reproduce the fully relativistic responses even for very high $q$ values (see, for example, [66]).

In this section we explore the impact of relativity on the MEC. 


\subsection{Pion-exchange currents}

We first compare the fully relativistic transverse MEC responses with the traditional non-relativistic calculation developed in [82], where the seagull $\mathrm{p}-\mathrm{h}$ matrix element is evaluated analytically, while the pion-in-flight contribution is reduced to a one-dimensional integral. For this comparison the value $\Gamma_{\pi}=1$ for the $\pi N N$ form factor and the static pion propagator have been used in the relativistic calculation. The effect of static versus dynamic pion propagator will be discussed later on.

From Fig. 23 it emerges that the two calculations give the same results for small density and momentum transfer. As $q$ and $k_{\mathrm{F}}$ increase we see that, apart from the difference stemming from the relativistic kinematics, which shrinks the response domain, the relativistic responses are smaller than the non-relativistic ones: this reduction amounts to about $30 \%$ for $q=500 \mathrm{MeV} / c$ and $k_{\mathrm{F}}=250 \mathrm{MeV} / c$, indicating that relativity plays an important role even for not so high $q$ values.

The same curves are displayed for $k_{\mathrm{F}}=250 \mathrm{MeV} / c$ and $q=500,600$ and $700 \mathrm{MeV} / c$ in Fig. 24, where it is shown that the effect of relativity clearly grows with the momentum transfer.

In Fig. 25 the relativistic MEC-correlated transverse response (dotted) is compared with the corresponding non-relativistic one (dot-dashed) as well as with the relativistic (solid) and non-relativistic (dashed) one-body response for three values of $q$. The figure shows that for low values of $q$ $(500 \mathrm{MeV} / c)$ the effects of MEC and relativity are roughly of the same size, the former acting mainly to the left of the QEP, the latter to the right. As $q$ increases, the effect of relativity becomes dominant, pointing to the necessity of a relativistic treatment for momentum transfers larger than $500 \mathrm{MeV} / c$.

Finally the impact on the responses of the relativistic propagator $\Delta_{\pi}(K)=\left(K^{2}-m_{\pi}^{2}\right)^{-1}$ as compared with the static one $\Delta_{\pi}^{(\text {n.r. })}(\mathbf{k})=-\left(\mathbf{k}^{2}+m_{\pi}^{2}\right)^{-1}$, which is commonly used in non-relativistic calculations, is explored. In Fig. 26 the pion-in-flight, seagull and total MEC contributions to $R^{\mathrm{T}}$ are evaluated for $q=0.5$ and $2 \mathrm{GeV} / c$ using the two versions of the propagator. It appears that the dynamical propagator affects the pion-in-flight contribution more than the seagull term (it increases the former by more than a factor 2 at $q=2 \mathrm{GeV} / c$ ); however, the two effects tend to cancel, so that their net effect is not very significant.

\subsubsection{The $\eta_{\mathrm{F}}$ expansion}

In view of the relevance of relativistic effects illustrated above and following the ideas and methods developed in the case of the single-nucleon electromagnetic current operator and its non-relativistic reduction [66], a new SR reduction of the MEC has been developed in [20], where the transferred energy and momentum have been left unexpanded while expanding only the initial nucleon momentum. The expressions thus obtained retain important aspects of relativity not included in the traditional non-relativistic MEC used throughout the literature. Here we summarize the basic results.

We are interested in the evaluation of the particle-hole matrix elements $\left\langle p k\left|j_{\text {MEC }}^{\mu}\right| k h\right\rangle$ and their new SR expressions. The resulting expansion for the MEC should be used together with the single-nucleon current, developed to first order in $\eta$ (see [20] and Appendix F), to set up the various responses. Therefore, in order to be consistent, the expansion of the MEC should also be performed to first order in the corresponding small quantities $\left\{\eta_{k} \equiv k / m, \eta_{h} \equiv h / m\right\}$, whereas $\left\{\eta_{p} \equiv p / m, \kappa \equiv q / 2 m\right\}$ are treated exactly.

After some algebra [20] the following SR expressions of the MEC currents (referred to as NR1 approximation in [20]) are obtained: 
Seagull
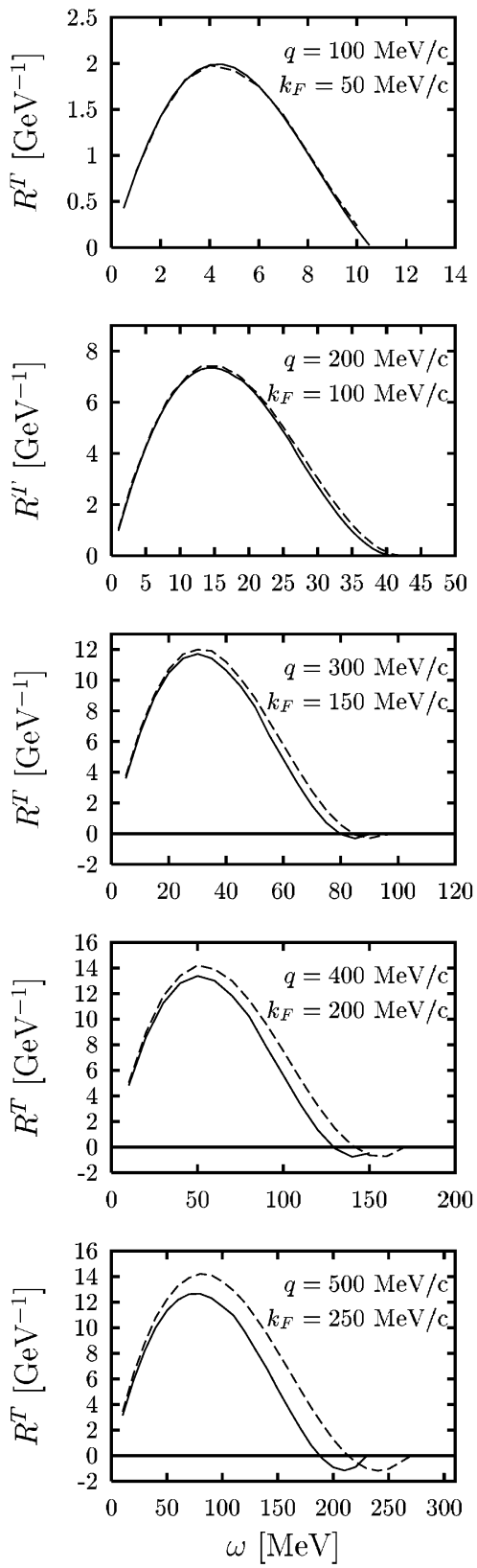

Pion in flight
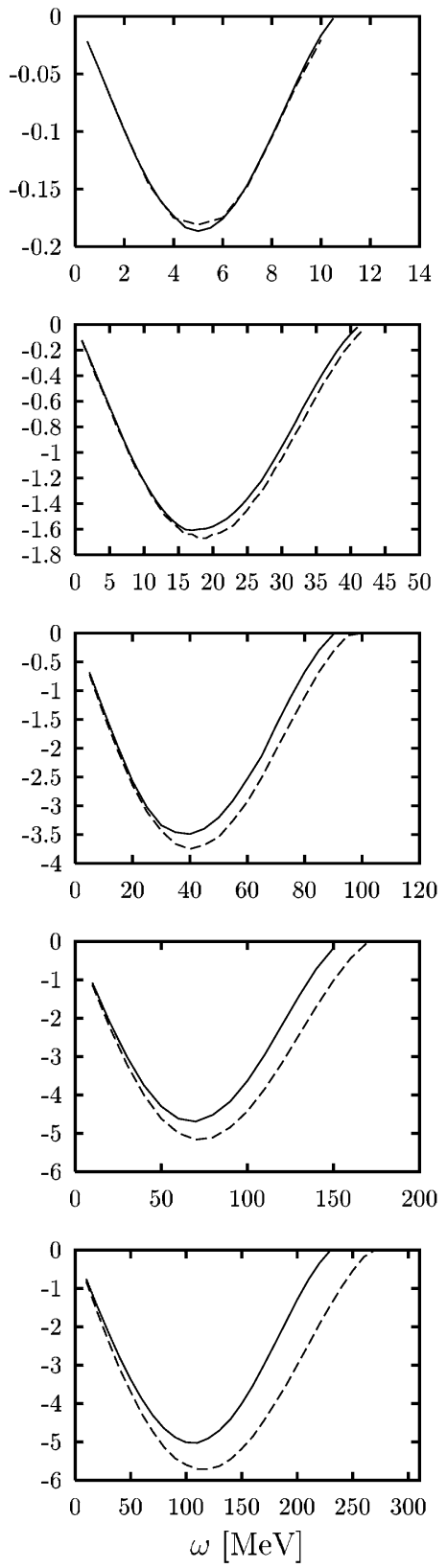

Fig. 23. The seagull (left panels) and pion-in-flight (right panels) contributions to the transverse response displayed versus $\omega$ for various values of the momentum transfer $q$ and of the Fermi momentum $k_{\mathrm{F}}$. Dashed: non-relativistic; solid: relativistic. 


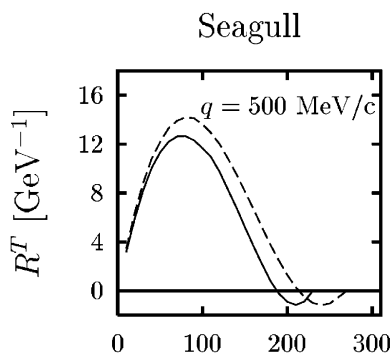

Pion in flight
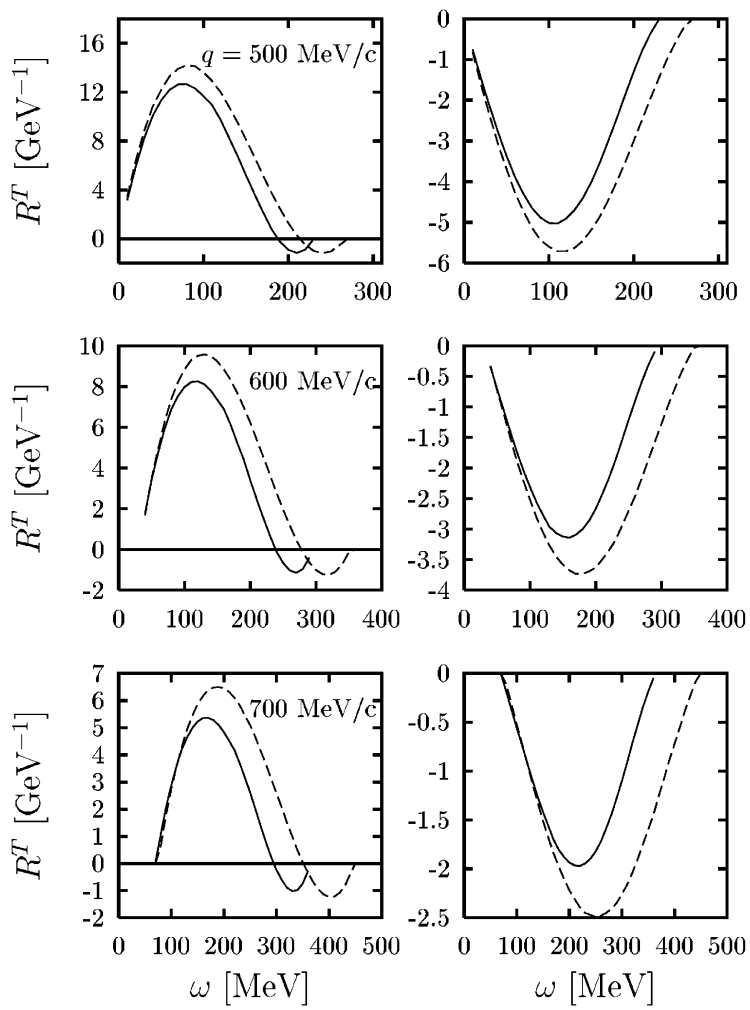

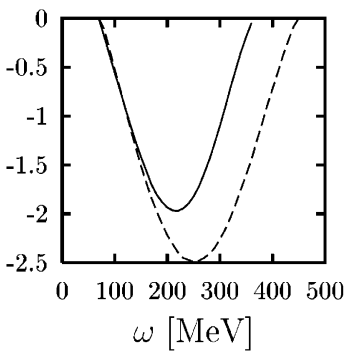

MEC
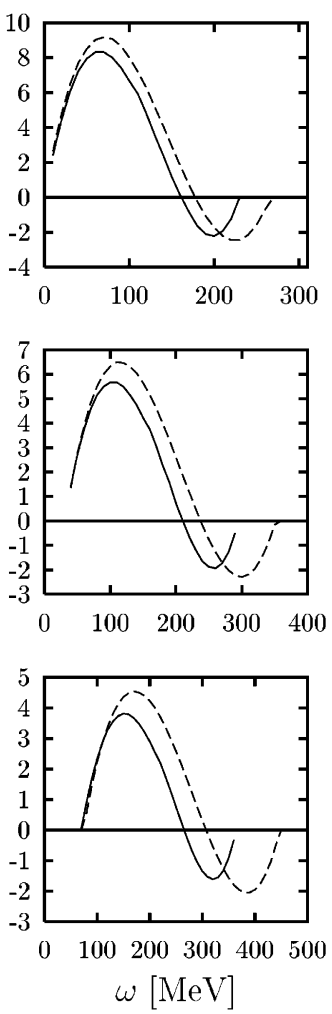

Fig. 24. The seagull (left panels), pion-in-flight (central panels) and total MEC (right panels) contributions to the transverse response displayed versus $\omega$ for $k_{\mathrm{F}}=250 \mathrm{MeV} / c$ and various values of the momentum transfer $q$. Dashed: non-relativistic; solid: relativistic.

\section{Seagull current operator.}

$j_{\mathrm{S}}^{0}(\mathbf{p}, \mathbf{k}, \mathbf{k}, \mathbf{p})_{\mathrm{SR} 1}$

$$
\begin{aligned}
= & \frac{\mathscr{F}}{2 \sqrt{1+\tau}} \chi_{s_{p}}^{\dagger}\left\{\frac{\boldsymbol{\sigma} \cdot\left[2 \boldsymbol{\kappa}+\boldsymbol{\eta}_{h}-(1+\tau) \boldsymbol{\eta}_{k}\right] \chi_{s_{k}} \chi_{s_{k}}^{\dagger} \boldsymbol{\sigma} \cdot\left(\boldsymbol{\eta}_{k}+\boldsymbol{\eta}_{h}\right)}{(P-K)^{2}-m_{\pi}^{2}}\right. \\
& \left.-\frac{\boldsymbol{\sigma} \cdot\left[2 \boldsymbol{\kappa}+\boldsymbol{\eta}_{h}+(1+\tau) \boldsymbol{\eta}_{k}\right] \chi_{s_{k}} \chi_{s_{k}}^{\dagger} \boldsymbol{\sigma} \cdot\left(\boldsymbol{\eta}_{k}-\boldsymbol{\eta}_{h}\right)}{(K-H)^{2}-m_{\pi}^{2}}\right\} \chi_{s_{h}},
\end{aligned}
$$

$\mathbf{j}_{\mathrm{s}}(\mathbf{p}, \mathbf{k}, \mathbf{k}, \mathbf{p})_{\mathrm{SR} 1}$

$$
\begin{aligned}
= & \frac{\mathscr{F}}{\sqrt{1+\tau}} \chi_{s_{p}}^{\dagger}\left\{\left[2 \boldsymbol{\sigma} \cdot \boldsymbol{\kappa}\left(1-\frac{\boldsymbol{\kappa} \cdot \boldsymbol{\eta}_{h}}{2(1+\tau)}\right)+\boldsymbol{\sigma} \cdot\left(\boldsymbol{\eta}_{h}-\boldsymbol{\eta}_{k}\right)-\tau \boldsymbol{\sigma} \cdot \boldsymbol{\eta}_{k}\right] \chi_{s_{k}}\right. \\
& \left.\times \chi_{s_{k}}^{\dagger} \frac{\boldsymbol{\sigma}}{(P-K)^{2}-m_{\pi}^{2}}-(1+\tau) \frac{\boldsymbol{\sigma}}{(K-H)^{2}-m_{\pi}^{2}} \chi_{s_{k}} \chi_{s_{k}}^{\dagger} \boldsymbol{\sigma} \cdot\left(\boldsymbol{\eta}_{k}-\boldsymbol{\eta}_{h}\right)\right\} \chi_{s_{h}},
\end{aligned}
$$



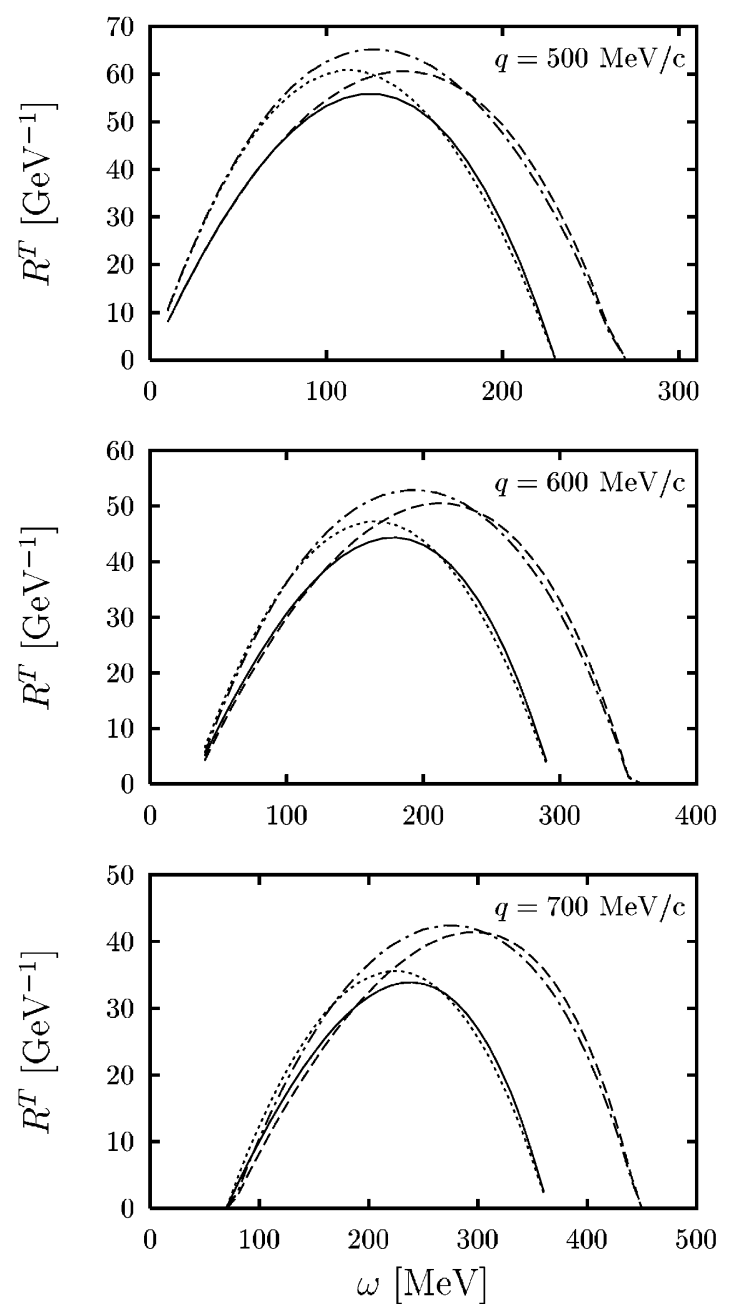

Fig. 25. The transverse response displayed versus $\omega$ for $k_{\mathrm{F}}=250 \mathrm{MeV} / c$ and various values of the momentum transfer $q$. Dashed: one-body non-relativistic; solid: RFG; dot-dashed: one-body+MEC non-relativistic; dotted: RFG+MEC relativistic.

where the factor

$$
\mathscr{F}=-\frac{f^{2} m}{m_{\pi}^{2}} \mathrm{i} \varepsilon_{3 a b}\left\langle t_{p}\left|\tau_{a}\right| t_{k}\right\rangle\left\langle t_{k}\left|\tau_{b}\right| t_{h}\right\rangle F_{1}^{\mathrm{V}}
$$

has been introduced. Note that if the terms $\boldsymbol{\eta}_{h}-(1+\tau) \boldsymbol{\eta}_{k}$ and $\boldsymbol{\eta}_{h}+(1+\tau) \boldsymbol{\eta}_{k}$ are neglected (this approximation will be referred to as SR2) the expression for the time component is similar to the one obtained in the traditional non-relativistic reduction [66] except for the factor $1 / \sqrt{1+\tau}$, which accordingly incorporates important aspects of relativity not considered in the traditional non-relativistic reduction. Analogously, in the space component, if the terms $\left(\boldsymbol{\kappa} \cdot \boldsymbol{\eta}_{h}\right) /[2(1+\tau)]$ and $\tau\left(\boldsymbol{\sigma} \cdot \boldsymbol{\eta}_{k}\right)$ are 

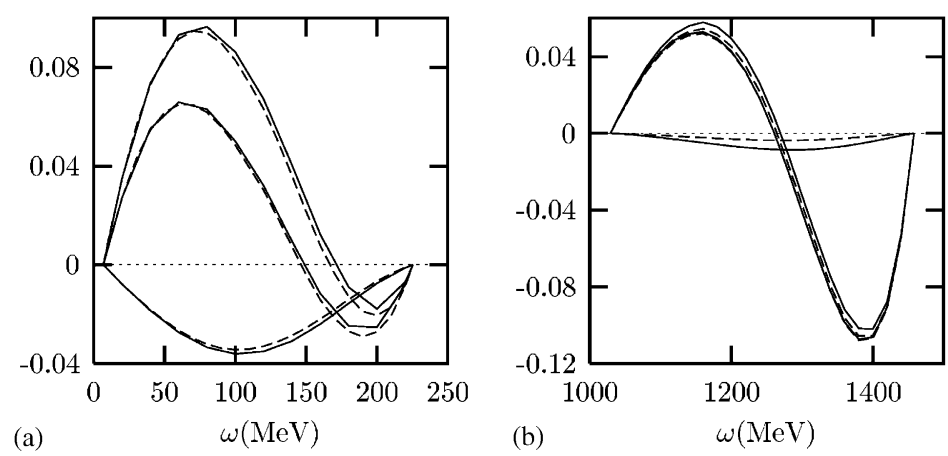

Fig. 26. MEC contribution to $R^{\mathrm{T}}$ (in $\mathrm{GeV}^{-1}$ ) versus $\omega$ with dynamic (solid curves) and static (dashed curves) pion propagator at (a) $q=0.5$ and (b) $2 \mathrm{GeV} / c$. The separate pion-in-flight and seagull contributions are displayed.

neglected (SR2 approximation), the traditional non-relativistic expression [24] is simply recovered, except for the factors $1 / \sqrt{1+\tau}$ and $\sqrt{1+\tau}$ that multiply the contributions given by the two diagrams involved. Thus, as in the case of the time component, also here important relativistic effects are simply accounted for by these multiplicative factors.

To illustrate this point we plot in Fig. 27 the current matrix element $K_{\mu}^{\mathrm{s}}$ defined through

$$
\frac{1}{V} \sum_{k<k_{\mathrm{F}}} \frac{m}{E_{\mathbf{k}}} j_{\mu}^{\mathrm{s}}(\mathbf{p}, \mathbf{k}, \mathbf{k}, \mathbf{h})=-\frac{m^{2}}{\sqrt{E_{\mathbf{p}} E_{\mathbf{h}} E_{\mathbf{k}}^{2}}} \frac{k_{\mathrm{F}}^{2}}{m} K_{\mu}^{\mathrm{s}}(q, \omega, \mathbf{h}) \sum_{t_{k}} \mathscr{F}
$$

for $q=1 \mathrm{GeV} / c, h=175 \mathrm{MeV} / c$ and $\phi_{h}=0$. The curves represent the fully relativistic result (solid), the traditional non-relativistic approximation, including relativistic kinematics through Eq. (172) (dot-dashed), the SR1 approximation of Eqs. (173) and (174) (dashed) and the SR2 approximation (dotted). Only the relevant components are shown, the other vanishing for symmetry reasons (see [20] for details). It clearly appears that, while the traditional non-relativistic reduction, although corrected by the replacement $\lambda \rightarrow \lambda(1+\lambda)$, fails to reproduce the exact results by roughly $10-20 \%$ (this deviation increasing with $q$, as shown in [20]), both the SR1 and SR2 approaches yield excellent agreement with the fully relativistic current.

Finally, we examine the limit $\eta_{\mathrm{F}} \rightarrow 0$, since this provides some understanding of how the MEC effects are expected to evolve in going from light $\left(\eta_{\mathrm{F}}\right.$ very small) to heavy nuclei $\left(\eta_{\mathrm{F}} \cong 0.29\right)$. In this limit the seagull current simply reduces to

$$
\lim _{\eta_{\mathrm{F}} \rightarrow 0} j_{\mathrm{S}}^{0}(\mathbf{p}, \mathbf{k}, \mathbf{k}, \mathbf{h})=0 .
$$

This is because the time component of the seagull current is of first order in the small momenta involved or, equivalently, it is $O\left(\eta_{\mathrm{F}}\right)$. On the contrary, the vector component in the limit $\eta_{\mathrm{F}} \rightarrow 0$ becomes

$$
\lim _{\eta_{\mathrm{F}} \rightarrow 0} \mathbf{j}_{\mathrm{s}}(\mathbf{p}, \mathbf{k}, \mathbf{k}, \mathbf{h})=\frac{2 \mathscr{F}}{\sqrt{1+\tau}} \chi_{s_{p}}^{\dagger} \frac{(\boldsymbol{\sigma} \cdot \boldsymbol{\kappa}) \chi_{s_{k}} \chi_{s_{k}}^{\dagger} \boldsymbol{\sigma}}{Q^{2}-m_{\pi}^{2}} \chi_{s_{h}},
$$

which shows that the space components of the seagull current are $O(1)$ and contribute even for nucleons at rest, as happens for the charge and magnetization pieces of the one-body current. 


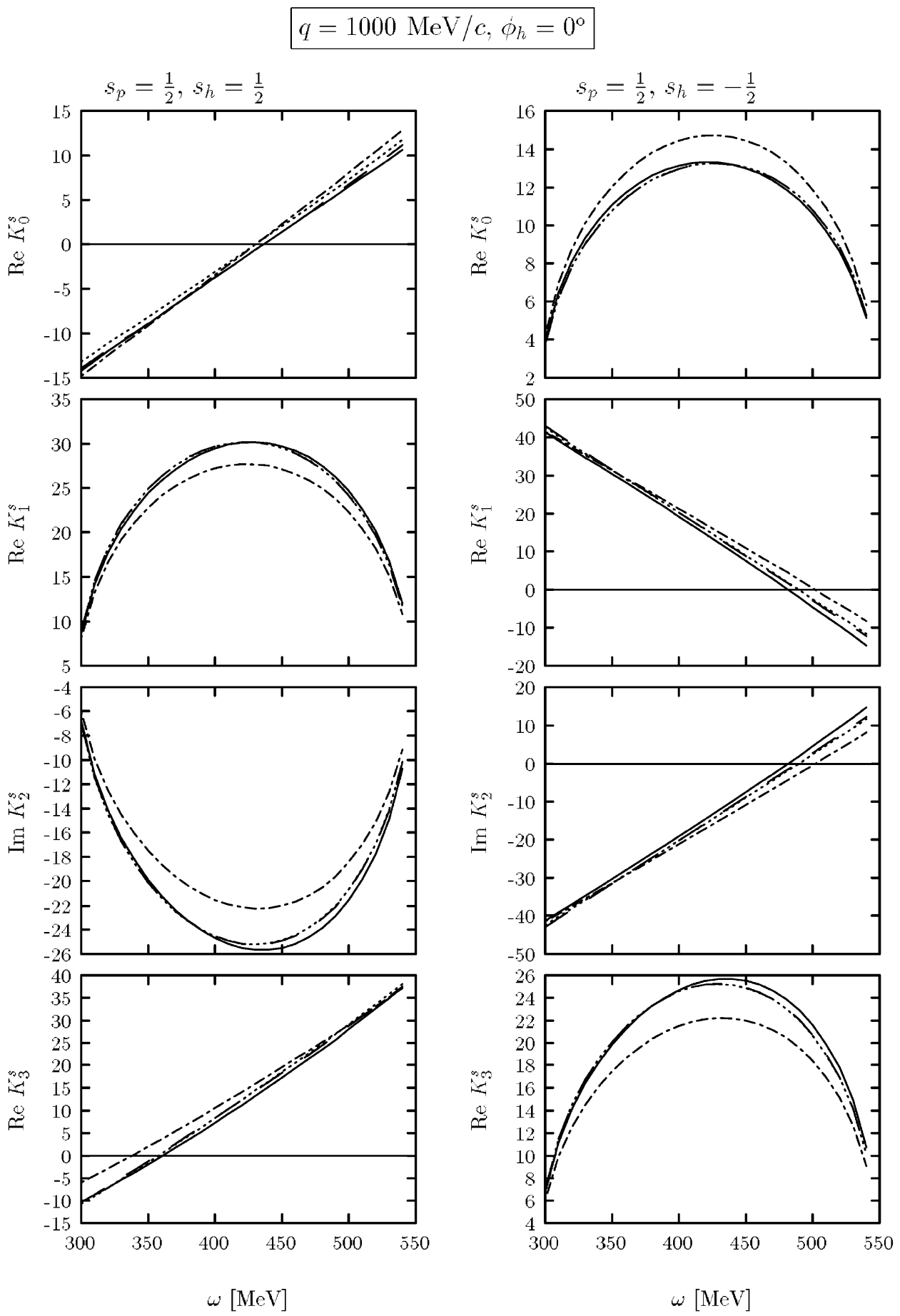

Fig. 27. Seagull current matrix element $K_{\mu}^{\mathrm{s}}$-see Eq. (176)-for $q=1 \mathrm{GeV} / c$ and $k_{\mathrm{F}}=250 \mathrm{MeV} / c$. The kinematics for the hole are $h=175 \mathrm{MeV} / c$ and $\phi_{h}=0$. First column: spin $\left(\frac{1}{2}, \frac{1}{2}\right)$ component; second column: spin $\left(\frac{1}{2},-\frac{1}{2}\right)$ component. Solid: fully relativistic; dashed: SR1 approximation; dot-dashed: traditional non-relativistic; dotted: SR2 approximation. 
Pion-in-flight current operator. Keeping only linear terms in the small momenta, one obtains for the SR pion-in-flight current

$$
\begin{aligned}
j_{\mathrm{p}}^{0}(\mathbf{p}, \mathbf{k}, \mathbf{k}, \mathbf{h})_{\mathrm{SR}} & =-\frac{\mathscr{F}}{\sqrt{1+\tau}} 4 m^{2} \tau \chi_{s_{p}}^{\dagger} \frac{\boldsymbol{\sigma} \cdot \boldsymbol{\kappa} \chi_{s_{k}} \chi_{s_{k}}^{\dagger} \boldsymbol{\sigma} \cdot\left(\boldsymbol{\eta}_{k}-\boldsymbol{\eta}_{h}\right)}{\left[(P-K)^{2}-m_{\pi}^{2}\right]\left[(K-H)^{2}-m_{\pi}^{2}\right]} \chi_{s_{h}}, \\
\mathbf{j}_{\mathrm{p}}(\mathbf{p}, \mathbf{k}, \mathbf{k}, \mathbf{h})_{\mathrm{SR}} & =-\frac{\mathscr{F}}{\sqrt{1+\tau}} 4 m^{2} \chi_{s_{p}}^{\dagger} \frac{\boldsymbol{\sigma} \cdot \boldsymbol{\kappa} \chi_{s_{k}} \chi_{s_{k}}^{\dagger} \boldsymbol{\sigma} \cdot\left(\boldsymbol{\eta}_{k}-\boldsymbol{\eta}_{h}\right)}{\left[(P-K)^{2}-m_{\pi}^{2}\right]\left[(K-H)^{2}-m_{\pi}^{2}\right]} \chi_{s_{h}} \boldsymbol{\kappa} .
\end{aligned}
$$

Again, these expressions are similar to the traditional non-relativistic currents [24] except for the common factor $1 / \sqrt{1+\tau}$, which includes important aspects of relativity not taken into account in the traditional non-relativistic reduction. Note that the space component of the pionic current is, in leading order, purely longitudinal; its transverse components are in fact of second order in $\eta_{\mathrm{F}}$.

In Fig. 28 we display the current matrix element $K_{\mu}^{\mathrm{p}}$, defined analogously to eq. (176), for $q=1 \mathrm{GeV} / c, h=175 \mathrm{MeV} / c$ and $\phi_{h}=0$. As for the seagull, the fully relativistic result (solid) is very well approximated by the SR prescription (dashed), whereas the traditional non-relativistic approach with relativistic kinematics (dot-dashed) deviates from the exact result by $10-20 \%$.

Finally the limit $\eta_{\mathrm{F}} \rightarrow 0$ implies that $j_{\mathrm{p}}^{\mu}(\mathbf{p}, \mathbf{k}, \mathbf{k}, \mathbf{h})=0$, since all components of the pionic current are $O\left(\eta_{\mathrm{F}}\right)$ in the expansion.

Summarizing, the $\eta$ expansion shows that relativity can be very easily implemented in MEC SR calculations by applying the prescription $\lambda \rightarrow \lambda(1+\lambda)$ and by multiplying the exchange currents by the kinematical factors indicated in Eqs. (173)-(180).

\subsubsection{Results for the responses using the relativized MEC model}

In this section we discuss the validity of the relativizing prescriptions introduced above, when they are implemented in a traditional non-relativistic model of the reaction. We begin with the non-relativistic Fermi gas of [82], which includes also MEC in the transverse response. One of the advantages of this model is that the integral over the Fermi sea appearing in the seagull matrix elements can be performed analytically, while the pion-in-flight is reduced to an one-dimensional integral.

Next we will relativize this model by implementing relativistic kinematics through the substitution $\lambda \rightarrow \lambda(1+\lambda)$ in all places except in the nucleon and pion form factors $F(q, \omega)$, which should be evaluated at the correct $\omega$-value. Second, we use the new SR expansion of the electromagnetic $\mathrm{OB}+\mathrm{MEC}$ operators in powers of $\eta$. For the OB operators we use the following expressions (see Appendix F):

$$
\begin{aligned}
& \rho_{\mathrm{OB}}=\frac{\kappa}{\sqrt{\tau}} G_{E}+\mathrm{i} \frac{G_{\mathrm{M}}-G_{E} / 2}{\sqrt{1+\tau}}(\boldsymbol{\kappa} \times \boldsymbol{\eta}) \cdot \boldsymbol{\sigma}, \\
& \mathbf{J}_{\mathrm{OB}}^{\mathrm{T}}=\frac{\sqrt{\tau}}{\kappa}\left[\mathrm{i} G_{\mathrm{M}}(\boldsymbol{\sigma} \times \boldsymbol{\kappa})+G_{E} \boldsymbol{\eta}_{T}\right] .
\end{aligned}
$$

Note that near the QEP it makes little difference to use the factors $1+\tau$ or $\kappa^{2} / \tau$. In these factors lies the main difference with the traditional non-relativistic charge and transverse current operators. Note that in addition we include a first-order spin-orbit term in the charge operator. The contribution of this term is small in the longitudinal unpolarized response, since its interference with the leading 


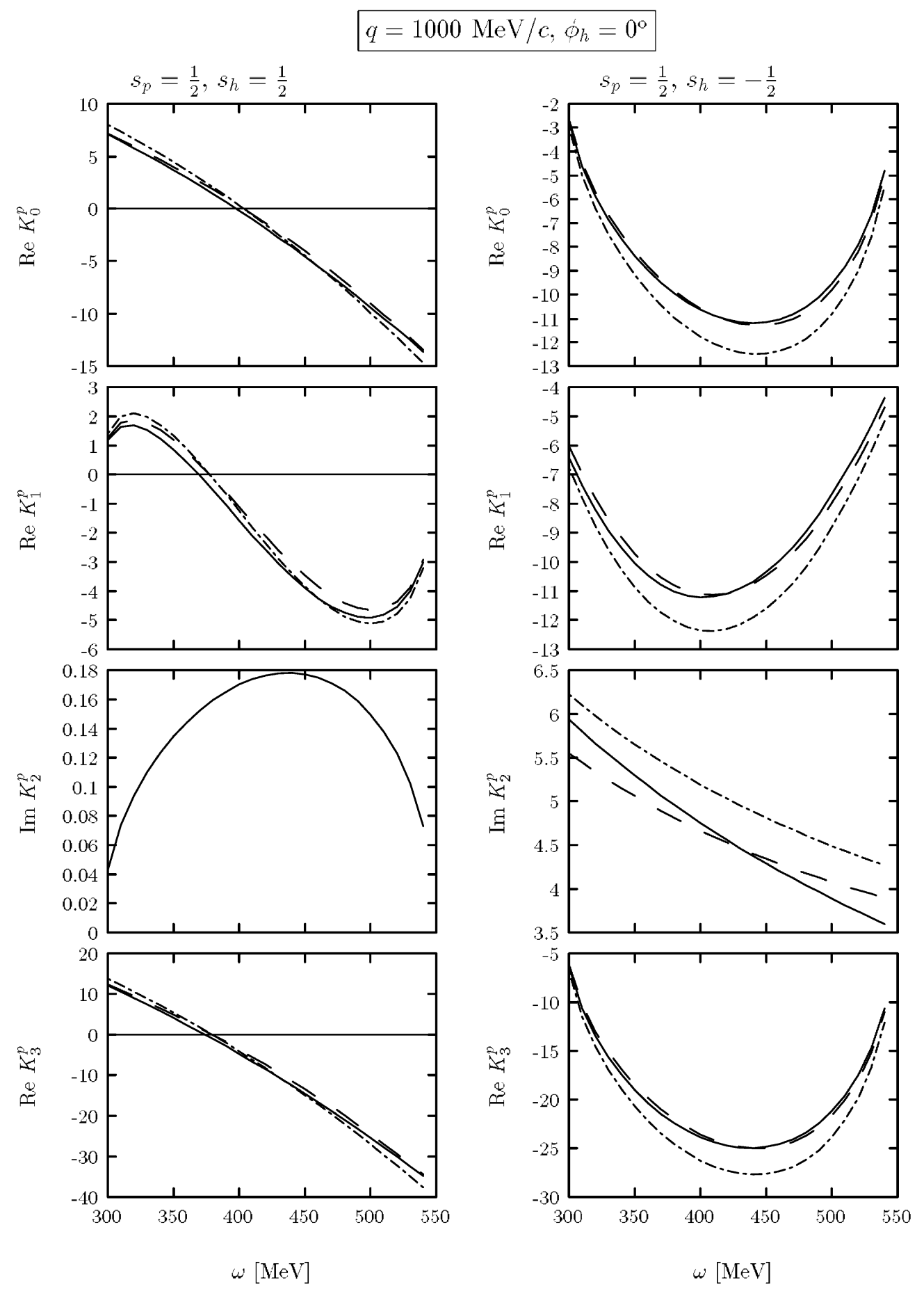

Fig. 28. Pion-in flight current matrix element $K_{\mu}^{\mathrm{p}}$ for $q=1 \mathrm{GeV} / c$ and $k_{\mathrm{F}}=250 \mathrm{MeV} / c$. The kinematics for the hole are $h=175 \mathrm{MeV} / c$ and $\phi_{h}=0$. First column: spin $\left(\frac{1}{2}, \frac{1}{2}\right)$ component; second column: spin $(1 / 2,-1 / 2)$ component. Solid: fully relativistic; dashed: semi-relativistic approximation; dot-dashed: traditional non-relativistic. 
order is exactly zero in PWIA and hence it gives a negligible contribution of second order in $\eta$. However one should be careful in including this term in the more complex cases in which there is an interference TL response (when the nucleus is polarized or in the exclusive reactions $\left(e, e^{\prime} p\right)$, see $[75,76,81])$, and where it gives a significant contribution, since in this response the leading order is zero in PWIA.

In the case of the MEC we use the following simplified prescription to relativize transverse operators:

$$
\mathbf{J}_{\mathrm{MEC}}^{\mathrm{T}}=\frac{1}{\sqrt{1+\tau}} \mathbf{J}_{\mathrm{MEC}, \text { non rel }}^{\mathrm{T}},
$$

namely we introduce a factor $1 / \sqrt{1+\tau}$ to take into account relativistic corrections coming from the free Dirac spinors. Note that in the case of the seagull we have neglected a further correction factor $1+\tau$ in the hole part of the seagull current. However here we choose to present results with the above simplified version of the transverse current, since it is easier to implement in already existing models of the reaction; otherwise one has to identify the different pieces of the operator, which may be difficult. Furthermore, this correction is not of much importance, its main effect being to correct slightly the position of the zero in the seagull response. Be it as it may this ad hoc prescription for the seagull current is supported by the quality of the results shown below.

Results for the ${ }^{40} \mathrm{Ca}$ nucleus for $q=500$ and $1000 \mathrm{MeV} / c$ are shown in Figs. 29 and 30, respectively. In the upper part of these figures we show the one-body (OB) separated longitudinal and transverse responses. The solid lines are the exact relativistic results. These are very different from the traditional non-relativistic results shown with dashed lines. Note that the same nucleon form factors and the same $k_{\mathrm{F}}=237 \mathrm{MeV} / c$ are used in both calculations. If we include relativistic kinematics, then we obtain the dotted lines, which are still different from the exact result, even if now the region where the response is non-zero is similar to the relativistic case. Finally, using in addition the new SR corrections (factors $\kappa / \sqrt{\tau}$ in the charge and $\sqrt{\tau} / \kappa$ in the current) we obtain the relativistic approximation shown with dot-dashed lines, which is very similar to the exact result. Hence we can safely say that the new expansion of the OB current is very good, giving essentially the exact answer.

The case of the MEC transverse responses is shown in the lower part of Figs. 29 and 30. There we show the separate contribution of seagull and pion-in-flight currents to the transverse response (interference with the OB current). Again we show with solid lines the exact relativistic results, and with dashed lines the traditional non-relativistic results. If again we include the relativistic kinematics we obtain the dotted lines. With dot-dashed lines we display results which include in addition the relativistic correction to the $\mathrm{OB}$ current, amounting to a factor $1 / \sqrt{1+\tau} \simeq \sqrt{\tau} / \kappa$. This correction produces a small reduction of the responses. Finally, with double-dashed lines we show the results computed using in addition the relativistic corrections in the MEC, which amounts to another factor $1 / \sqrt{1+\tau}$. This correction produces a further reduction of the responses, giving a result which is closer to the exact one.

From these results it appears that our expansion of MEC currents is not as good (at least fractionally) as the $\mathrm{OB}$ expansion. This is likely related to the fact that the $\mathrm{OB}$ currents have been expanded in powers of $\eta=h / M$, where $h$ is the momentum of the hole, and they are exact, by construction, for $h=0$. However in the case of the MEC there is another variable in the expansion: 

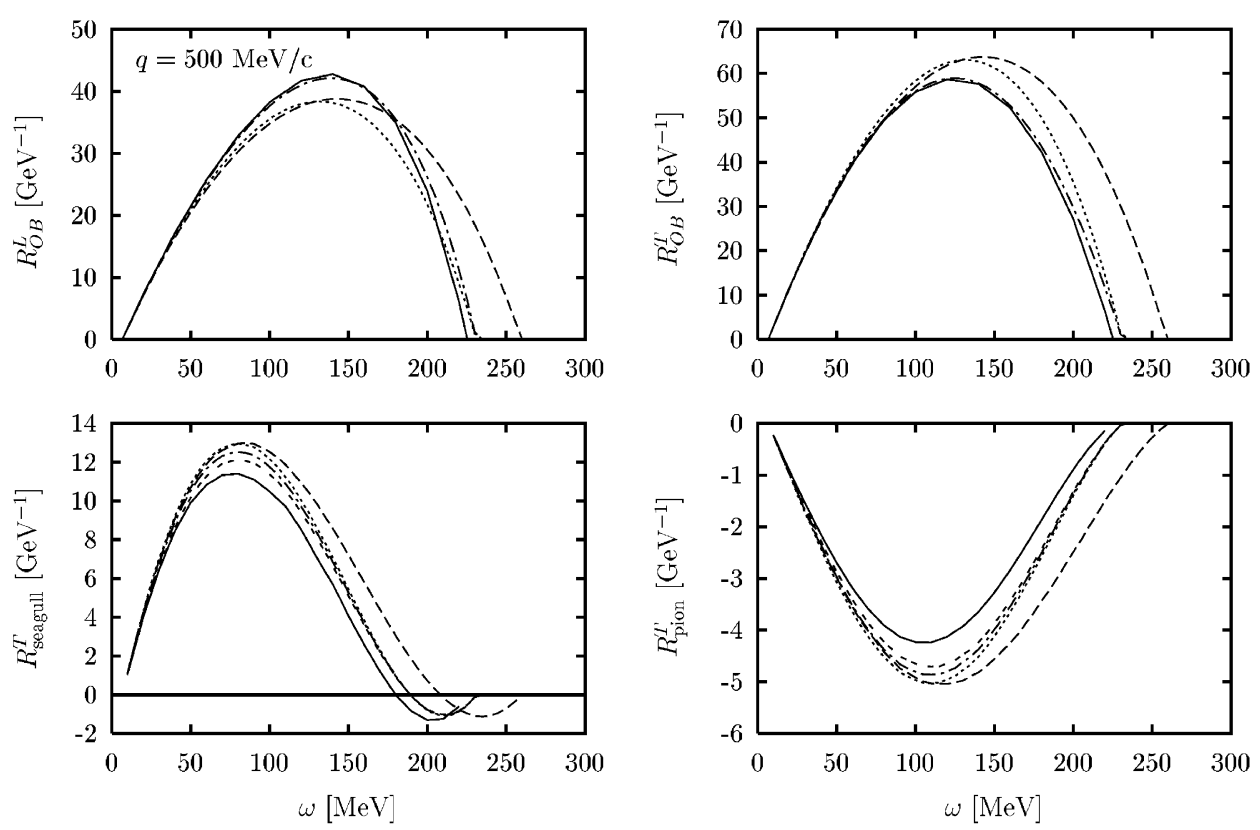

Fig. 29. One-body longitudinal and transverse response functions (top panels), and transverse responses of interference between MEC and $\mathrm{OB}$ currents (bottom panels) for $q=500 \mathrm{MeV} / c$ and $k_{\mathrm{F}}=237 \mathrm{MeV} / c$. Solid: exact relativistic results with static propagator and without $\pi N$ form factor. The rest of the curves have been computed using the non-relativistic Fermi gas model, with or without relativistic corrections. Dashed: traditional non-relativistic results. Dotted: including relativistic kinematics in the non-relativistic calculations. Dot-dashed: including in addition the new expansion of the OB currents. Double-dashed: including in addition a correction to the MEC operators with a factor $1 / \sqrt{1+\tau}$.

the momentum of the second hole $h^{\prime} / M$, which is small, but is never zero and is being integrated up to $k_{\mathrm{F}}$. Therefore our currents are not constructed to agree with the exact ones for $h=0$.

On the other hand, we have explicitly showed before that in the limit $q \rightarrow 0$ and $k_{\mathrm{F}} \rightarrow 0$, the relativistic and non-relativistic results agree. This is also the case for the present results of the relativized currents, as it is illustrated in Fig. 31. There we show the seagull and pionic responses for several small values of $q=100, \ldots, 500 \mathrm{MeV} / c$ and for $k_{\mathrm{F}}=q / 2$. With solid lines we show the exact relativistic results, while with dashed lines we show the traditional non-relativistic results. Finally we also show with dotted lines the results using the present semi-relativized approach. It is seen that the last are always much closer to the exact result than the non-relativistic ones, and that they converge faster to the exact results.

Better agreement between the exact and the relativized models for the MEC responses is also expected in the limit $\eta_{\mathrm{F}} \rightarrow 0$ in the quasielastic peak, since in this case both momenta $h$ and $h^{\prime}$ are forced to be small, which are the conditions assumed in our expansion. Results for the transverse MEC responses in this limit are shown in Fig. 32 for $q=1000 \mathrm{MeV} / c$ and for two values of $k_{\mathrm{F}}=50$ and $25 \mathrm{MeV} / c$. With solid lines we show the exact relativistic results, while with dashed lines we display the non-relativistic ones, but including relativistic kinematics. Finally, the dotted lines correspond to the semi-relativized results, which fully agree with the exact ones in the case of the seagull current, while in the pionic case there is still a difference between the two calculations. 

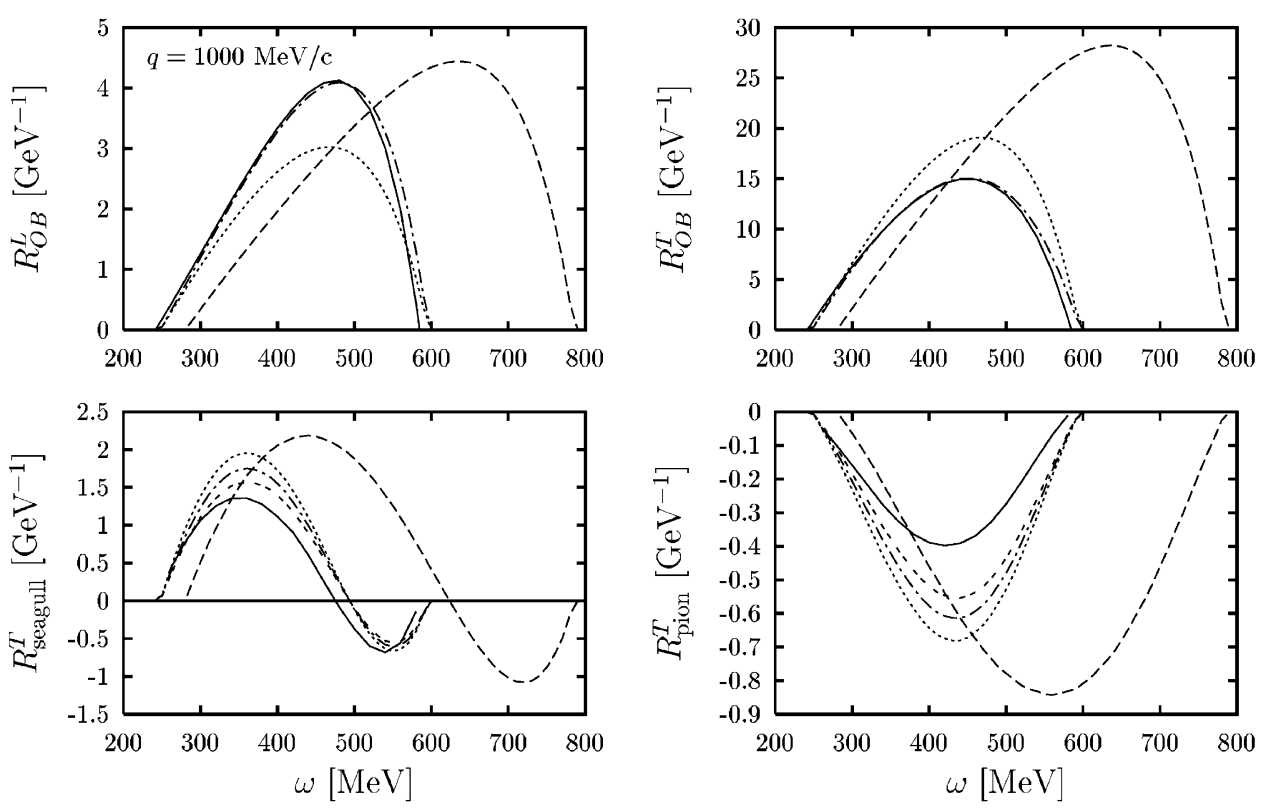

Fig. 30. The same as Fig. 29, now for $q=1000 \mathrm{MeV} / c$.

However this is not very important in this limit, since the transverse pionic contribution which we are computing here is of second order in the hole momenta, and so is negligible compared with the seagull one, as can be seen in the figure.

In order to improve the present results for the MEC responses one should look for an expansion of the MEC in the form

$$
\mathbf{J}(p, h)=\frac{N\left(q, \omega, k_{\mathrm{F}}\right)}{\sqrt{1+\tau}} \mathbf{J}_{\text {non rel }}(p, h),
$$

where $N\left(q, \omega, k_{\mathrm{F}}\right)$ is an appropriate normalization factor defined by requiring

$$
\lim _{h \rightarrow 0} \frac{\mathbf{J}(p, h)}{\mathbf{J}_{\text {rel }}(p, h)}=1,
$$

i.e., the coincidence between the relativistic and the approximate results at the quasielastic peak. Obviously the factor $N\left(q, \omega, k_{\mathrm{F}}\right)$ is a function of $k_{\mathrm{F}}$ also, since an integral over the Fermi sphere is implicit in the definition of the MEC in the $1 \mathrm{p}-1 \mathrm{~h}$ channel, and it can be written in the form

$$
N\left(q, \omega, k_{\mathrm{F}}\right)=\sqrt{1+\tau} \frac{\mathbf{J}_{\text {rel }}(q, 0)}{\mathbf{J}_{\text {non rel }}(q, 0)} .
$$

A simple approximation for this function is not easy to obtain, since it requires the knowledge of the exact relativistic answer.

Despite these difficulties, the quality of the OB expansion plus the approximated improvement of the MEC currents obtained in the present expansion are good enough to ensure a quite satisfactory description of the exact relativistic transverse response using the relativized OB plus MEC operators 
Seagull
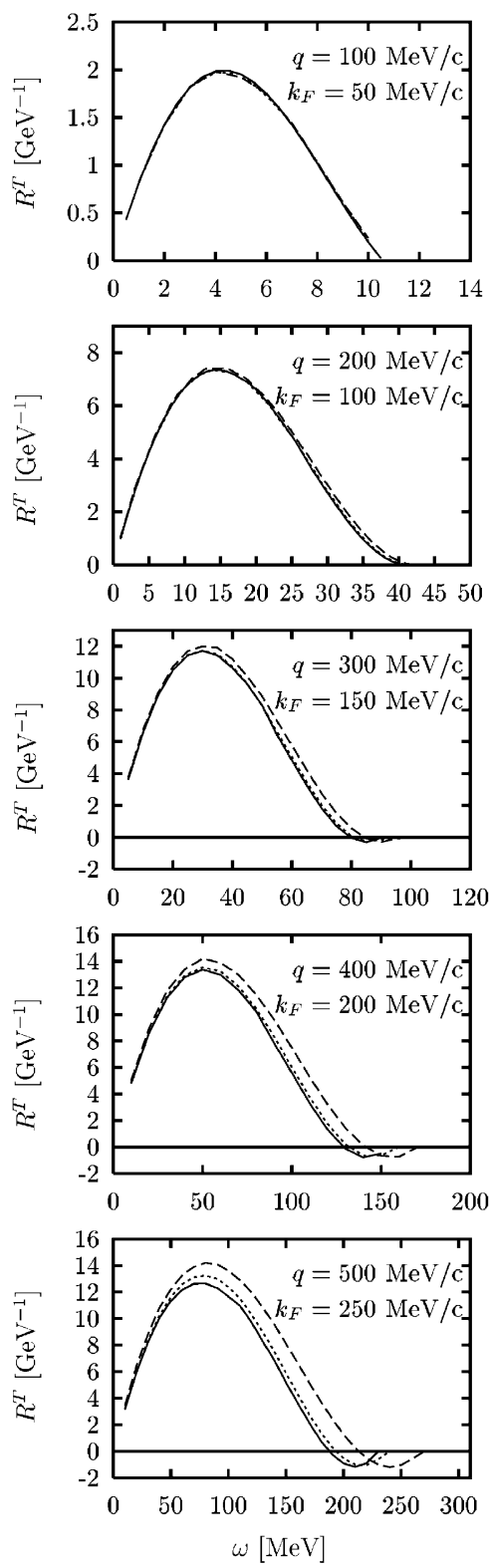

Pion in flight
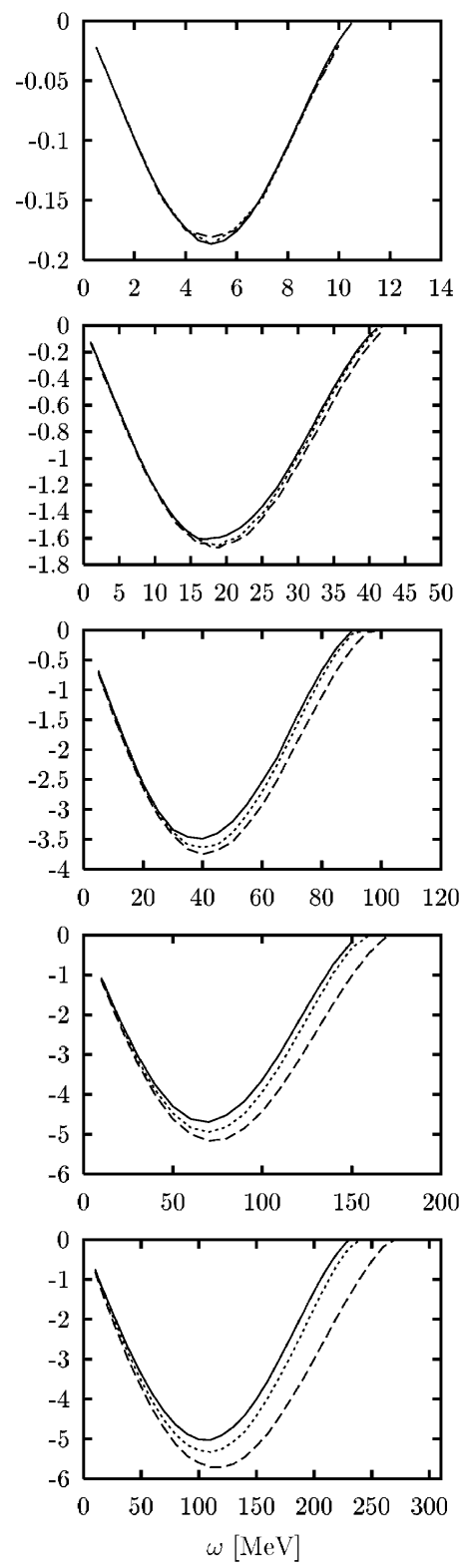

Fig. 31. Seagull and pionic responses computed for several values of $q$ and $k_{\mathrm{F}}$. Solid lines: exact relativistic results. Dashed lines: non-relativistic results. Dotted lines: approximated semi-relativistic results using relativistic kinematics and relativized currents. Static propagators without a $\pi N N$ form factor have been used here.

altogether with relativistic kinematics. This is shown in Fig. 33, where we show the total transverse response, including $\mathrm{OB}+\mathrm{MEC}$ operators, for $q=500,1000,2000$ and $3000 \mathrm{MeV} / c$. The solid lines are the exact relativistic result. Again with dashed lines we display the traditional non-relativistic 

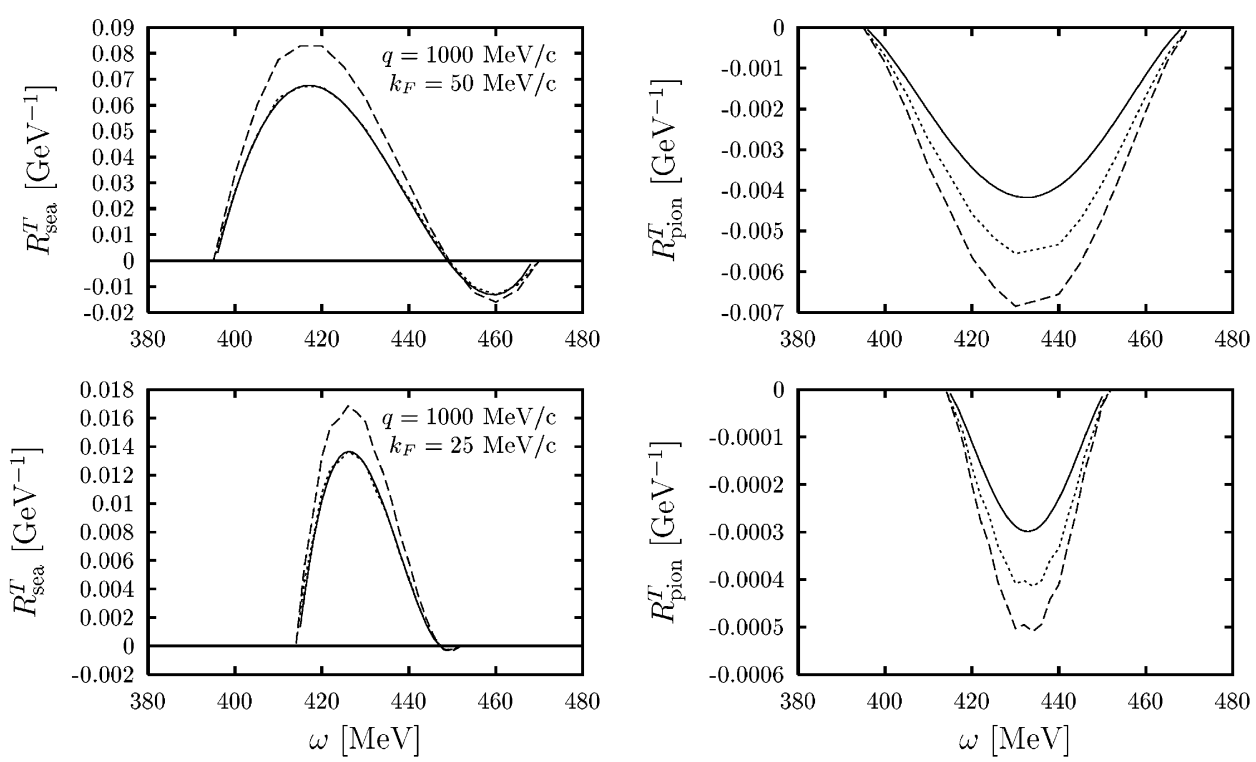

Fig. 32. MEC-OB transverse responses for $q=1000 \mathrm{MeV} / c$ and $k_{\mathrm{F}}=50 \mathrm{MeV} / c$. Solid: exact relativistic results with static propagator and without a $\pi N$ form factor. Dashed: non-relativistic results, but including relativistic kinematics. Dotted: using in addition the relativized currents.

results, which together with relativistic kinematics give the dotted lines. Finally, with dot-dashed lines we show the results using the semi-relativized OB+MEC currents. The agreement between the two models is quite good even for very high $q$, since the major part of the relativistic effects is included in the approximated model, and therefore these currents are very appropriate and easy to implement in already existing non-relativistic models of the reaction.

\subsubsection{Comparison with the traditional relativistic corrections}

Here we discuss the reasons why the present expansion of electromagnetic operators is preferable to other kinds of relativistic corrections existing in the literature. The most common of these is the Darwin-Foldy correction to the charge operator of the nucleus [7,83-85]. This correction is usually derived from a Foldy-Wouthuysen transformation [31], but can also be obtained from a Pauli reduction of the spin matrix element (we do not write the spin indices for simplicity):

$$
J_{E}^{\mu}(\mathbf{p}, \mathbf{h})=\bar{u}_{E}(\mathbf{p}) \Gamma^{\mu}(Q) u_{E}(\mathbf{h})=\bar{u}_{E}(\mathbf{p})\left(F_{1} \gamma^{\mu}+\mathrm{i} \frac{F_{2}}{2 m} \sigma^{\mu v} Q_{v}\right) u_{E}(\mathbf{h}),
$$

where we use the sub-index $E$ to denote the spinors normalized to $u_{E}^{\dagger}(\mathbf{p}) u_{E}(\mathbf{p})=1$, i.e., namely

$$
u_{E}(\mathbf{p})=\left(\frac{E+m}{2 E}\right)^{1 / 2}\left(\begin{array}{c}
\chi \\
\frac{\boldsymbol{\sigma} \cdot \mathbf{p}}{E+m}
\end{array}\right) .
$$

This is in contrast to the Bjorken and Drell spinor normalization used in the present work, where spinors are normalized to $u^{\dagger}(\mathbf{p}) u(\mathbf{p})=E / m$. The relation between the two sets of spinors 

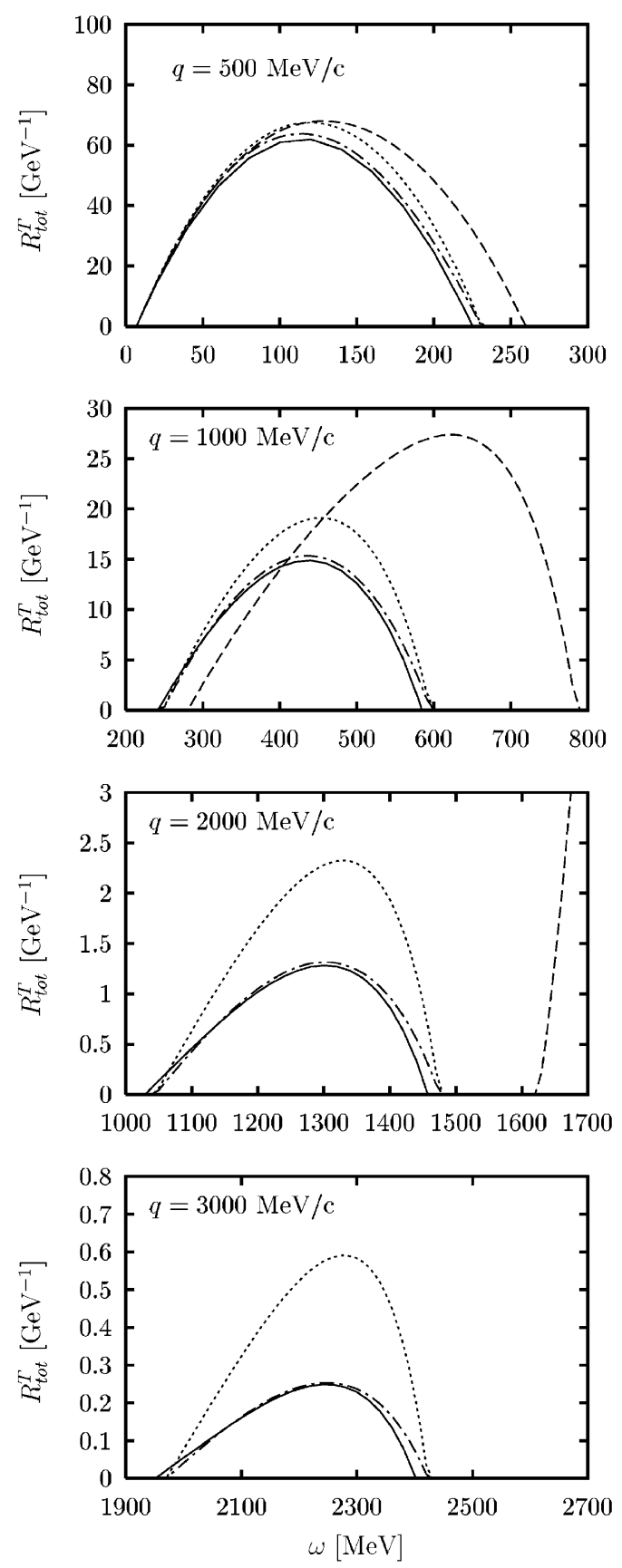

Fig. 33. Total transverse response function of ${ }^{40} \mathrm{Ca}$ including $\mathrm{MEC}$ for several values of the momentum transfer, and for $k_{\mathrm{F}}=237 \mathrm{MeV} / c$. Solid: exact relativistic results. The rest of the curves have been computed using the non-relativistic Fermi gas model, with or without relativistic corrections. Dashed: traditional non-relativistic results. Dotted: including relativistic kinematics in the non-relativistic calculations. Dot-dashed: including in addition the new expansion of the OB+MEC currents. The relativistic calculations include a dynamical propagator and $\pi N$ form factor, while the non-relativistic calculations do not include these corrections. 
obviously is

$$
u(\mathbf{p})=\sqrt{\frac{E}{m}} u_{E}(\mathbf{p}) .
$$

Of course both formalisms based on the different spinor sets $u_{E}(\mathbf{p})$ or $u(\mathbf{p})$ should give the same results for the observable quantities. For instance, if we like to think in terms of wave functions, for a nucleon in a box of volume $V$ this would be

$$
\psi(x)=\sqrt{\frac{m}{E V}} u(\mathbf{p}) \mathrm{e}^{-\mathrm{i} p \cdot x}=\frac{1}{\sqrt{V}} u_{E}(\mathbf{p}) \mathrm{e}^{-\mathrm{i} p \cdot x} .
$$

This means that observables (expectation values, probabilities or cross sections) computed using the Bjorken and Drell normalization always contain additional phase-space factors $m / E$, while these factors do not appear explicitly if one uses the $E$-scheme, since they are already included inside the spinors $u_{E}(\mathbf{p})$.

As an example let us consider the case of the longitudinal response function for protons

$$
\begin{aligned}
R^{\mathrm{L}} & =\sum_{\mathbf{p h}} \sum_{s_{p} s_{h}} \delta\left(E_{\mathbf{p}}-E_{\mathbf{h}}-\omega\right)\left|\left\langle p h^{-1}|\rho(\mathbf{q})| F\right\rangle\right|^{2} \\
& =\sum_{\mathbf{p h}} \delta\left(E_{\mathbf{p}}-E_{\mathbf{h}}-\omega\right) \frac{m^{2}}{E_{\mathbf{p}} E_{\mathbf{h}}} \delta_{\mathbf{p}, \mathbf{h}+\mathbf{q}} \operatorname{Tr}\left[\rho(\mathbf{p}, \mathbf{h})^{\dagger} \rho(\mathbf{p}, \mathbf{h})\right] \\
& =\left(\frac{3 Z}{8 \pi k_{\mathrm{F}}^{3}}\right) \int_{h<k_{\mathrm{F}}} \mathrm{d}^{3} h \delta\left(E_{\mathbf{p}}-E_{\mathbf{h}}-\omega\right) \frac{m^{2}}{E_{\mathbf{p}} E_{\mathbf{h}}} \operatorname{Tr}\left[\rho(\mathbf{p}, \mathbf{h})^{\dagger} \rho(\mathbf{p}, \mathbf{h})\right],
\end{aligned}
$$

where in the last line $\mathbf{p}=\mathbf{h}+\mathbf{q}$, and we have used the replacement $\sum_{\mathbf{h}} \rightarrow\left(V /(2 \pi)^{3}\right) \int \mathrm{d}^{3} h$, with $V /(2 \pi)^{3}=3 Z / 8 \pi k_{\mathrm{F}}^{3}$. Note that we use the Bjorken and Drell normalization and so the energy denominators appear explicitly. The charge matrix element used here is the fully relativistic one $\rho(\mathbf{p}, \mathbf{h})=\bar{u}(\mathbf{p}) \Gamma^{0}(Q) u(\mathbf{h})$.

The interesting (and crucial) point is that the energy denominator $E_{\mathbf{p}}$ cancels out when we perform the integral over $\cos \theta$ - the angle between $\mathbf{h}$ and $\mathbf{q}-$ using the energy-conserving delta function. In fact, from

$$
E_{\mathbf{p}}^{2}=\mathbf{p}^{2}+m^{2}=\mathbf{h}^{2}+\mathbf{q}^{2}+2 h q \cos \theta+m^{2},
$$

we have $E_{\mathbf{p}} \mathrm{d} E_{\mathbf{p}}=h q \mathrm{~d} \cos \theta$. Therefore, the angle $\theta$ becomes fixed by the energy conservation $E_{\mathbf{p}}=$ $E_{\mathbf{h}}+\omega$ and we obtain

$$
\begin{aligned}
R^{\mathrm{L}}(q, \omega) & =\frac{3 Z}{8 \pi k_{\mathrm{F}}^{3}} \int_{0}^{k_{\mathrm{F}}} h \mathrm{~d} h \int_{0}^{2 \pi} \mathrm{d} \phi \frac{E_{\mathbf{p}}}{q} \frac{m^{2}}{E_{\mathbf{p}} E_{\mathbf{h}}} \operatorname{Tr}\left[\rho(\mathbf{p}, \mathbf{h})^{\dagger} \rho(\mathbf{p}, \mathbf{h})\right] \\
& =\frac{3 Z}{8 \pi k_{\mathrm{F}}^{3}} \frac{m}{q} \int_{0}^{k_{\mathrm{F}}} h \mathrm{~d} h \int_{0}^{2 \pi} \mathrm{d} \phi \frac{m}{E_{\mathbf{h}}} \operatorname{Tr}\left[\rho(\mathbf{p}, \mathbf{h})^{\dagger} \rho(\mathbf{p}, \mathbf{h})\right] .
\end{aligned}
$$

This expression has to be compared with the non-relativistic response function, which can be computed by repeating the steps above using instead the non-relativistic energies $\epsilon_{\mathbf{p}}=\mathbf{p}^{2} / 2 \mathrm{~m}$, 
i.e.,

$$
R^{\mathrm{L}}(q, \omega)_{\text {non rel }}=\left(\frac{3 Z}{8 \pi k_{\mathrm{F}}^{3}}\right) \int_{h<k_{\mathrm{F}}} \mathrm{d}^{3} h \delta\left(\epsilon_{\mathbf{p}}-\epsilon_{\mathbf{h}}-\omega\right) \operatorname{Tr}\left[\rho(\mathbf{p}, \mathbf{h})^{\dagger} \rho(\mathbf{p}, \mathbf{h})\right]_{\text {n.r. }},
$$

where again $\mathbf{p}=\mathbf{h}+\mathbf{q}$, no energy denominators appear and the non-relativistic charge operator is used.

Now the integral over $\cos \theta$ can again be performed using the non-relativistic identity

$$
\epsilon_{\mathbf{p}}=\frac{\mathbf{p}^{2}}{2 m}=\frac{\mathbf{h}^{2}+\mathbf{q}^{2}+2 h q \cos \theta}{2 m} .
$$

Hence $\mathrm{d} \epsilon_{\mathbf{p}}=(h q / m) \mathrm{d} \cos \theta$ and the integral over the new variable $\epsilon_{\mathbf{p}}$ can be performed. The latter is $\epsilon_{\mathbf{p}}=\epsilon_{\mathbf{h}}+\omega$. We obtain

$$
R^{\mathrm{L}}(q, \omega)_{\text {nonrel }}=\frac{3 Z}{8 \pi k_{\mathrm{F}}^{3}} \frac{m}{q} \int_{0}^{k_{\mathrm{F}}} h \mathrm{~d} h \int_{0}^{2 \pi} \mathrm{d} \phi \operatorname{Tr}\left[\rho(\mathbf{p}, \mathbf{h})^{\dagger} \rho(\mathbf{p}, \mathbf{h})\right]_{\text {n.r. }},
$$

which has formally the same structure as Eq. (193) with the exception of the factor $m / E_{\mathbf{h}} \simeq 1$ included there. Therefore, the relativistic response can be reproduced using a non-relativistic model if we introduce in the non-relativistic response in Eq. (196) a good approximation for $\rho(\mathbf{p}, \mathbf{h})$, and in addition we use relativistic kinematics, i.e., we use the relativistic relation between $\cos \theta$ and $\omega$. This can be approximately accomplished starting from Eq. (192). Indeed we have

$$
\begin{aligned}
h^{2}+q^{2}+2 h q \cos \theta & =\left(E_{\mathbf{h}}+\omega\right)^{2}-m^{2}=h^{2}+\omega^{2}+2 E_{\mathbf{h}}+\omega \\
& \simeq h^{2}+\omega^{2}+2 m \omega+2 \epsilon_{\mathbf{h}} \omega \\
& =2 m\left(\omega+\epsilon_{\mathbf{h}}+\frac{\omega^{2}+2 \epsilon_{\mathbf{h}} \omega}{2 m}\right) \\
& \simeq 2 m\left(\omega+\epsilon_{\mathbf{h}}+\frac{\omega^{2}}{2 m}\right),
\end{aligned}
$$

where we have neglected the term $\epsilon_{\mathbf{h}} \omega / m=O\left(h^{2} / m^{2}\right)$. Comparing with the non-relativistic relation (195) we see that the relativistic one can be approximately obtained with the replacement $\omega \rightarrow$ $\omega(1+\omega / 2 m)$. The validity of this approximate method of relativization was demonstrated numerically in the last sections.

If instead we use the other spinor normalization $u_{E}(\mathbf{p})$ in the relativistic model, then the matrix element is $\rho_{E}(\mathbf{p}, \mathbf{h})$ and now there are no explicit energy denominators in the expression of the response, which hence becomes, after integration over $\theta$,

$$
R^{\mathrm{L}}(q, \omega)=\frac{3 Z}{8 \pi k_{\mathrm{F}}^{3}} \frac{m}{q} \int_{0}^{k_{\mathrm{F}}} h \mathrm{~d} h \int_{0}^{2 \pi} \mathrm{d} \phi \frac{E_{\mathbf{p}}}{m} \operatorname{Tr}\left[\rho_{E}(\mathbf{p}, \mathbf{h})^{\dagger} \rho_{E}(\mathbf{p}, \mathbf{h})\right] .
$$

Comparing with Eq. (196) we see that if we start with a non-relativistic model and use an approximate non-relativistic form for $\rho_{E}(\mathbf{p}, \mathbf{h})$, as happens with the Darwin-Foldy correction, an additional 
factor $E_{\mathbf{p}} / m$ is needed in order to reproduce the relativistic response. For this reason, a careless introduction of relativistic corrections alone in non-relativistic models can produce incorrect results.

\subsection{Pionic correlations}

The analysis carried out in the previous section for the MEC could in principle be extended to the correlation current. However in this case the calculation becomes extremely cumbersome and has not yet been performed. A SR calculation of the vertex and self-energy correlations has been carried out in $[51,55,86]$, where the relativistic energy-conserving delta function has been accounted for via the replacement in Eq. (172) and the form factors in the two-body current have been modified to implement relativistic effects. The response functions so obtained are in qualitative agreement with the fully relativistic ones for not too high $q$. However, for high values of $q$ a careful treatment of the relativistic effects is needed.

In what follows we briefly examine the non-relativistic limit of the vertex correlations and selfenergy diagrams in order to bring to light some differences with respect to the fully relativistic case.

The non-relativistic leading order of the pionic correlation currents in Eqs. (27) and (28) is obtained by using the following prescriptions, valid in the static limit:

$$
\begin{aligned}
& E_{\mathbf{k}} \simeq m \\
& \gamma_{5} K \simeq \boldsymbol{\sigma} \cdot \mathbf{k}, \\
& \frac{1}{K^{2}-m_{\pi}^{2}} \simeq-\frac{1}{\mathbf{k}^{2}+m_{\pi}^{2}}, \\
& S_{\mathrm{F}}(P) \simeq S_{\mathrm{nr}}(P)=\frac{1}{p_{0}-\mathbf{p}^{2} / 2 m} .
\end{aligned}
$$

The electromagnetic form factor $\Gamma^{\mu}(Q)$ is also replaced by $\Gamma_{\mathrm{nr}}^{\mu}(q)$, representing the usual nonrelativistic one-body current acting over bi-spinors [20,24]. Using the above relations and performing the sums over spin and isospin indices, the VC and SE current matrix elements read

$$
\begin{aligned}
j_{\mathrm{VC}}^{\mu}(\mathbf{p}, \mathbf{h})_{\mathrm{nr}}= & \frac{f^{2}}{V m_{\pi}^{2}} \chi_{s_{p}}^{\dagger} \sum_{\mathbf{k} \leqslant k_{\mathrm{F}}}\left\{\frac{\boldsymbol{\sigma} \cdot(\mathbf{k}-\mathbf{h})}{(\mathbf{k}-\mathbf{h})^{2}+m_{\pi}^{2}} S_{\mathrm{nr}}(K+Q) \tau_{a} \Gamma_{\mathrm{nr}}^{\mu}(Q) \tau_{a} \boldsymbol{\sigma} \cdot(\mathbf{k}-\mathbf{h})\right. \\
& \left.+\boldsymbol{\sigma} \cdot(\mathbf{p}-\mathbf{k}) \tau_{a} \Gamma_{\mathrm{nr}}^{\mu}(Q) \tau_{a} S_{\mathrm{nr}}(K-Q) \frac{\boldsymbol{\sigma} \cdot(\mathbf{p}-\mathbf{k})}{(\mathbf{p}-\mathbf{k})^{2}+m_{\pi}^{2}}\right\} \chi_{s_{h}}
\end{aligned}
$$

and

$$
j_{\mathrm{SE}}^{\mu}(\mathbf{p}, \mathbf{h})_{\mathrm{nr}}=\chi_{s_{p}}^{\dagger}\left[\Sigma_{\mathrm{nr}}(\mathbf{p}) S_{\mathrm{nr}}(P) \Gamma_{\mathrm{nr}}^{\mu}(Q)+\Gamma_{\mathrm{nr}}^{\mu}(Q) S_{\mathrm{nr}}(H) \Sigma_{\mathrm{nr}}(\mathbf{h})\right] \chi_{s_{h}},
$$

where $\chi_{s_{p}}$ and $\chi_{s_{h}}$ are two-components spinors. The non-relativistic self-energy function is given by

$$
\Sigma_{\mathrm{nr}}(\mathbf{p})=3 \frac{f^{2}}{V m_{\pi}^{2}} \sum_{\mathbf{k} \leqslant k_{\mathrm{F}}} \frac{(\mathbf{p}-\mathbf{k})^{2}}{(\mathbf{p}-\mathbf{k})^{2}+m_{\pi}^{2}}=\Sigma_{\mathrm{nr}}(|\mathbf{p}|) .
$$

The above expressions coincide with the traditional non-relativistic currents used in the literature. 
With the self-energy in Eq. (205) one can then construct the non-relativistic Fock nucleon propagator

$$
S_{\mathrm{nr}}^{\mathrm{HF}}\left(p_{0}, \mathbf{p}\right)=\frac{1}{p_{0}-\frac{\mathbf{p}^{2}}{2 m}}+\frac{1}{p_{0}-\frac{\mathbf{p}^{2}}{2 m}} \sum_{\mathrm{nr}}(\mathbf{p}) \frac{1}{p_{0}-\frac{\mathbf{p}^{2}}{2 m}}+\cdots=\frac{1}{p_{0}-\frac{\mathbf{p}^{2}}{2 m}-\Sigma_{\mathrm{nr}}(\mathbf{p})} .
$$

As is well known, this is a meromorphic function whose simple pole again defines the new energy of the nucleon in the medium, namely

$$
\varepsilon_{\mathrm{nr}}(\mathbf{p})=\frac{\mathbf{p}^{2}}{2 m}+\Sigma_{\mathrm{nr}}(\mathbf{p}),
$$

since $\Sigma_{\mathrm{nr}}(\mathbf{p})$ is a function only of $\mathbf{p}$.

Since the non-relativistic self-energy function in Eq. (205) does not depend on spin, the nucleon wave functions are not modified in the medium. In fact the corresponding Schrödinger equation in momentum space, including the self-energy, is simply given by

$$
\left[\frac{\mathbf{p}^{2}}{2 m}+\Sigma_{\mathrm{nr}}(\mathbf{p})\right] \phi_{\mathrm{nr}}(\mathbf{p})=p_{0} \phi_{\mathrm{nr}}(\mathbf{p}),
$$

with the bi-spinor $\phi_{\mathrm{nr}}(\mathbf{p})$ corresponding to the eigenvalue $p_{0}=\epsilon_{\mathrm{nr}}(\mathbf{p})$.

The non-relativistic analysis of the nucleon self-energy current [43] is much simpler than its relativistic counterpart. Indeed, in the former the self-consistency is immediately achieved because the nucleon wave functions are not modified by the self-energy interaction and thus the first iteration of the "Hartree-Fock" equations already provides the exact energy. By contrast, in the relativistic framework the spin dependence of the self-energy [87] modifies the Dirac spinors, inducing an enhancement of the lower components. Moreover, the field-strength renormalization constant, namely the residue of the nucleon propagator in Eq. (206) at the pole, in the non-relativistic case is just unity. Hence the enhancement of the lower components and the spinors' field strength renormalization are genuine relativistic effects absent in a non-relativistic analysis where only the energy-momentum relation in the medium is altered by the self-energy diagrams. We have shown in Section 2.4 that the two above-mentioned relativistic signatures can be incorporated as new pieces in the electromagnetic current acting over free spinors.

\section{Conclusions}

Our goal in these studies has been to explore some of the ingredients that enter at high energies where relativistic effects become important in attempting to model the nuclear response functions for inclusive quasielastic electron scattering. The full problem of accounting for relativistic dynamics in nuclear physics is a daunting one and far from being solved [88,89]. While in many papers it appears that a reasonable level of understanding has been reached [1,90-92], since the basic trends seen in the data are reproduced, closer scrutiny reveals a different situation. It is not only that contributions left out in various analyses are far from being small, but, even more serious, fundamental physics principles (Lorentz covariance, gauge invariance and unitarity) turn out patently to be violated. Thus the successes in reproducing the experiments often reflect more an adjusting of parameters than a real understanding of the physics involved in the quasielastic regime. 
Our approach has been less to use a highly elaborated non-relativistic model whose failings are expected at the outset than to employ a simple model in which the important consequences of relativity are hopefully present. For this we have begun with the relativistic Fermi gas as our starting point $[93,94]$. This approach is motivated by several critical features of the model, namely, that it is Lorentz covariant, that it allows the implementation of gauge invariance and that it is simple enough to be tractable and yet not obviously lacking at least for the quasielastic responses for which it is designed. Clearly it is not an appropriate way to proceed if near-Fermi-surface physics is the goal and this regime is not our focus.

With these as basic motivations in a series of papers we have explored the consequences of having a Lorentz covariant model. In particular in [20] we attempted to approximate the full theory by identifying a dimensionless variable that is small enough to be suitable in setting up a semi-relativistic expansion of the responses (namely the momentum of a nucleon lying below the Fermi surface compared with its mass). In contrast, in very recent work [19] no expansion whatsoever is involved and the theory is now fully relativistic.

Our treatment proceeds in terms of nucleonic and mesonic degrees of freedom (the latter viewed both as force and current carriers). As our aim is to study the quasielastic regime where the longest-range hadronic ingredients may be expected to be dominant, we focus on pions; studies using a larger set of hadrons can be undertaken and some steps have already been taken by us in that direction. In our model, the pions are dealt with to first order in a perturbative framework, since their effects on the free responses of the RFG are not expected to be too disruptive.

Gauge invariance is a fundamental property we have also addressed in very recent work [19]. We now understand how the continuity equation is satisfied order by order in perturbation theory. We have succeeded in showing that the continuity equation for the one-body (single-nucleon) and the two-body (MEC and correlations) currents is fulfilled, implying that our approach deals consistently with both forces and currents.

Given the point in our understanding of the quasielastic responses at relatively high energies, we have been motivated to provide a comprehensive set of discussions of progress made so far. In particular, as a more in-depth presentation of the analysis carried out in [19], where we first studied the fully relativistic set of OPE operators that contribute to the electromagnetic responses of nuclei in the $1 \mathrm{p}-1 \mathrm{~h}$ channel, in the present work we have gone further to answer the question of whether or not a finite OPE self-energy current in nuclear matter exists. Indeed we have proven that the latter can be obtained through a renormalization of the $1 \mathrm{p}-1 \mathrm{~h}$ excitation vertex with a Fock self-energy insertion in the particle or in the hole line. In [19] these diagrams were shown to diverge but, at the same time, to be crucial to preserve gauge invariance. To overcome this impasse in that work we abandoned the notion of current operators, using instead the polarization propagator for computation of these diagrams. Indeed the double pole appearing in the self-energy polarization propagator can be dealt with employing the derivative of the nucleon propagator.

In assessing the role of the pions in the electromagnetic nuclear responses, the MEC are not the only contributions that arise in first-order perturbation theory. In fact, the pionic correlations are intimately linked to MEC through the continuity equation and, as we have seen, only when the full set of Feynman diagrams with OPE is considered can one expect gauge invariance to be fulfilled.

Since all of these ingredients are required for a consistent theory, a question we have addressed in this paper is whether or not a unified treatment based on current operators at the level of the OPE can be used even for the self-energy contribution. We succeeded in achieving this goal introducing 
a new ingredient: the first-order correction to the wave function and energy of the nucleon in the medium, which is modified by the interaction with the other nucleons. Indeed the iteration of the self-energy diagrams generates a "dressed" propagator in the medium. By the same token, the self-energy generates "dressed" or "renormalized" wave functions in the medium, solutions of an in-medium Dirac equation, where the self-energy plays the role of a mean relativistic potential. This equation also provides the dispersion relation linking the energy and momentum of the nucleon in the medium. Importantly, the new spinors should be multiplied by a renormalization function $\sqrt{Z_{2}(\mathbf{p})}$.

As the self-energy is generated by the interaction of a nucleon with the other nucleons in the medium, the solutions of the new Dirac equation should be used as input to re-compute the self-energy and so on. The exact answer is obtained through a self-consistent procedure. In this paper, however, we have just considered the first iteration: we have thus computed the self-energy current confining ourselves to first-order corrections to the energy and spinors - or, equivalently, to corrections linear in the self-energy-which correspond to diagrams with only one pionic line, in order to be consistent with the MEC and vertex correlation currents.

Notably in the first-order expansion of the renormalized spinors two new elements with respect to the non-relativistic approach emerge, one arising from the negative-energy components in the wave function produced by the interaction, the other from the renormalization function $\sqrt{Z_{2}(\mathbf{p})}$. These two elements can be combined in a new renormalized self-energy current, $j_{\mathrm{RSE}}^{\mu}$, acting over free spinors, and, together with renormalized self-energies, lead to the same self-energy contribution of [19]. The introduction of renormalized energies produces a shift of the response function. Our results for the response functions for typical kinematics show that the negative energy components constitute a correction to the total self-energy contribution of roughly $10-20 \%$, whereas the renormalization function for OPE is small, yet necessary if gauge invariance is to be fulfilled exactly. Moreover, while at low momentum transfers both particle and hole contributions play a role in the response, at high $q$ only the hole contribution survives. Finally, the self-energy contribution to the response functions is comparable in size to the one arising from the MEC and vertex correlations.

These formal developments have been discussed at length in the present work, and not to interrupt the flow of the arguments unduly some details have been placed in a series of appendices.

In the remainder of the article we have presented some typical results, both for parity-conserving and parity-violating quasielastic electron scattering. Briefly we have found the following: we have found that the MEC contributions are small enough to be well handled in first order. In particular, both the pion-in-flight and seagull contributions are very small in the L channel where the virtual photon exchanged between the electron and the Fermi gas couples to the charge of the pion, implying as expected that the MEC only marginally affect the Coulomb sum rule. In contrast in the T channel the MEC are more significant. There the seagull contribution dominates, and one sees that the MEC contribution does not vanish when $q$ increases. In [19] the scaling behaviors of the MEC were also explored in detail: in summary it was seen that they break scaling of the second kind everywhere, but, while breaking scaling of the first kind at modest momentum transfers, tend to successful first-kind scaling behavior at sufficiently high values of $q$.

The correlation contributions arising from the vertex corrections (VC) display a different pattern: the $\mathrm{L}$ channel dominates over the $\mathrm{T}$ channel by an amount of roughly $3: 1$. Thus the longitudinal response effectively picks up only these correlation contributions, since the MEC effects are so small there, and the former contribute to the total at roughly the $10-15 \%$ level. Indeed, were these to be the only contributions needed in addition to the RFG response itself, then we would expect the 
total to shift in $\omega$. Note that, since the correlation contributions are roughly symmetrical about the quasielastic peak, their impact on the Coulomb sum rule should be very small, perhaps only at the few percent level. The correlation contribution to $R^{\mathrm{T}}$ is similar to the MEC contribution, but is smaller, roughly $\frac{1}{2}$ the size of the latter; since the two are of opposite sign, they tend to cancel and thus the total is similar to the MEC contribution but is cut down roughly by a factor of two.

In summary, the total contribution (the sum of $1 \mathrm{p}-1 \mathrm{~h}$ MEC $+1 \mathrm{p}-1 \mathrm{~h}$ correlations) to be added to the RFG response (1) is not insignificant, (2) is Lorentz covariant/gauge invariant and interestingly (3) does not go away as $q$ becomes very large.

\section{Acknowledgements}

J.E.A. would like to thank J. Nieves for useful discussions. This work was partially supported by funds provided by DGICYT (Spain) under Contract Nos. PB/98-1111, PB/98-0676 and PB/98-1367 and the Junta de Andalucía (Spain), by the Spanish-Italian Research Agreement HI1998-0241, by the "Bruno Rossi" INFN-CTP Agreement, by the INFN-CICYT exchange and in part by the US. Department of Energy under Cooperative Research Agreement No. DE-FC02-94ER40818.

\section{Appendix A. Gauge invariance of two-body currents}

In this appendix we prove that the total two-body current is gauge invariant at the level of the two-body matrix elements in free space. We start by evaluating the contraction of the four-momentum transfer $Q_{\mu}$ with the correlation current $j_{\text {cor }}^{\mu}\left(\mathbf{p}_{1}^{\prime}, \mathbf{p}_{2}^{\prime}, \mathbf{p}_{1}, \mathbf{p}_{2}\right)$. It can be written as

$$
Q_{\mu} j_{\text {cor }}^{\mu}\left(\mathbf{p}_{1}^{\prime}, \mathbf{p}_{2}^{\prime}, \mathbf{p}_{1}, \mathbf{p}_{2}\right)=\frac{f^{2}}{m_{\pi}^{2}} \bar{u}\left(\mathbf{p}_{1}^{\prime}\right) \tau_{a} \gamma_{5} K_{1} u\left(\mathbf{p}_{1}\right) \frac{1}{K_{1}^{2}-m_{\pi}^{2}} \mathscr{M}_{a}+(1 \leftrightarrow 2)
$$

with $\mathscr{M}_{a}$ given by

$$
\mathscr{M}_{a}=\bar{u}\left(\mathbf{p}_{2}^{\prime}\right)\left[\tau_{a} \gamma_{5} K_{1} S_{\mathrm{F}}\left(P_{2}+Q\right) / Q F_{1}+F_{1} / Q S_{\mathrm{F}}\left(P_{2}^{\prime}-Q\right) \tau_{a} \gamma_{5} K_{1}\right] u\left(\mathbf{p}_{2}\right),
$$

where we have used the relation $Q_{\mu} \Gamma^{\mu}(Q)=F_{1}(Q) / Q$. After some algebra, involving the nucleon propagator and the Dirac spinors, $\mathscr{M}_{a}$ can be further simplified leading to

$$
\mathscr{M}_{a}=\bar{u}\left(\mathbf{p}_{2}^{\prime}\right)\left[\tau_{a} \gamma_{5} K_{1} F_{1}-F_{1} \tau_{a} \gamma_{5} K_{1}\right] u\left(\mathbf{p}_{2}\right)=\bar{u}\left(\mathbf{p}_{2}^{\prime}\right)\left[\tau_{a}, F_{1}\right] \gamma_{5} K_{1} u\left(\mathbf{p}_{2}\right) .
$$

To evaluate the commutator $\left[\tau_{a}, F_{1}\right]$ we now decompose the nucleon form factor into its isoscalar and isovector pieces, $F_{1}=\frac{1}{2}\left(F_{1}^{\mathrm{S}}+F_{1}^{\mathrm{V}} \tau_{3}\right)$. Then

$$
\left[\tau_{a}, F_{1}\right]=-\mathrm{i} F_{1}^{\mathrm{V}} \varepsilon_{3 a b} \tau_{b},
$$

which entails the automatic conservation of the $\pi^{0}$ exchange current $(a=3)$. Using Eq. (A.4) we can recast $\mathscr{M}_{a}$ as follows:

$$
\mathscr{M}_{a}=-\mathrm{i} F_{1}^{\mathrm{V}} \varepsilon_{3 a b} \bar{u}\left(\mathbf{p}_{2}^{\prime}\right) \tau_{b} \gamma_{5} K_{1} u\left(\mathbf{p}_{2}\right) \text {. }
$$


Hence the divergence of the two-body correlation current matrix element can finally be written as

$$
\begin{aligned}
Q_{\mu} j_{\text {cor }}^{\mu}\left(\mathbf{p}_{1}^{\prime}, \mathbf{p}_{2}^{\prime}, \mathbf{p}_{1}, \mathbf{p}_{2}\right)= & 2 m \frac{f^{2}}{m_{\pi}^{2}} \mathrm{i} \varepsilon_{3 a b} \bar{u}\left(\mathbf{p}_{1}^{\prime}\right) \tau_{a} \gamma_{5} u\left(\mathbf{p}_{1}\right) \frac{F_{1}^{\mathrm{V}}}{K_{1}^{2}-m_{\pi}^{2}} \\
& \times \bar{u}\left(\mathbf{p}_{2}^{\prime}\right) \tau_{b} \gamma_{5}(\mathscr{Q}+2 m) u\left(\mathbf{p}_{2}\right)+(1 \leftrightarrow 2) .
\end{aligned}
$$

The divergence of the seagull and pion-in-flight two-body current matrix elements can also be calculated in a straightforward way. The final result reads

$$
\begin{aligned}
Q_{\mu} j_{\mathrm{s}}^{\mu}\left(\mathbf{p}_{1}^{\prime}, \mathbf{p}_{2}^{\prime}, \mathbf{p}_{1}, \mathbf{p}_{2}\right)= & -2 m \frac{f^{2}}{m_{\pi}^{2}} \mathrm{i} \varepsilon_{3 a b} \bar{u}\left(\mathbf{p}_{1}^{\prime}\right) \tau_{a} \gamma_{5} u\left(\mathbf{p}_{1}\right) \frac{F_{1}^{\mathrm{V}}}{K_{1}^{2}-m_{\pi}^{2}} \\
& \times \bar{u}\left(\mathbf{p}_{2}^{\prime}\right) \tau_{b} \gamma_{5} Q u\left(\mathbf{p}_{2}\right)+(1 \leftrightarrow 2) \\
Q_{\mu} j_{\mathrm{p}}^{\mu}\left(\mathbf{p}_{1}^{\prime}, \mathbf{p}_{2}^{\prime}, \mathbf{p}_{1}, \mathbf{p}_{2}\right)= & 4 m^{2} \frac{f^{2}}{m_{\pi}^{2}} \mathrm{i} \varepsilon_{3 a b} F_{\pi} \frac{\left(K_{1}-K_{2}\right) \cdot Q}{\left(K_{1}^{2}-m_{\pi}^{2}\right)\left(K_{2}^{2}-m_{\pi}^{2}\right)} \\
& \times \bar{u}\left(\mathbf{p}_{1}^{\prime}\right) \tau_{a} \gamma_{5} u\left(\mathbf{p}_{1}\right) \bar{u}\left(\mathbf{p}_{2}^{\prime}\right) \tau_{b} \gamma_{5} u\left(\mathbf{p}_{2}\right) .
\end{aligned}
$$

Then, by summing up the contributions given by the correlation (Eq. (A.6)) and seagull (Eq. (A.7)) currents and writing the four-momentum transfer as $Q_{\mu}=\left(K_{1}+K_{2}\right)_{\mu}$, we finally obtain

$$
\begin{aligned}
Q_{\mu} & {\left[j_{\text {cor }}^{\mu}\left(\mathbf{p}_{1}^{\prime}, \mathbf{p}_{2}^{\prime}, \mathbf{p}_{1}, \mathbf{p}_{2}\right)+j_{\mathrm{s}}^{\mu}\left(\mathbf{p}_{1}^{\prime}, \mathbf{p}_{2}^{\prime}, \mathbf{p}_{1}, \mathbf{p}_{2}\right)\right] } \\
& =4 m^{2} \frac{f^{2}}{m_{\pi}^{2}} F_{1}^{\mathrm{V}} \mathrm{i} \varepsilon_{3 a b} \frac{\left(K_{2}-K_{1}\right) \cdot Q}{\left(K_{1}^{2}-m_{\pi}^{2}\right)\left(K_{2}^{2}-m_{\pi}^{2}\right)} \bar{u}\left(\mathbf{p}_{1}^{\prime}\right) \tau_{a} \gamma_{5} u\left(\mathbf{p}_{1}\right) \bar{u}\left(\mathbf{p}_{2}^{\prime}\right) \tau_{b} \gamma_{5} u\left(\mathbf{p}_{2}\right),
\end{aligned}
$$

which cancels exactly the contribution of pion-in-flight current in Eq. (A.8) provided the electromagnetic pion form factor is chosen to be $F_{\pi}=F_{1}^{\mathrm{V}}$.

\section{Appendix B. Gauge invariance of the two-body current $\mathrm{p}-\mathrm{h}$ matrix elements}

Following the study of gauge invariance at the level of the free-space particle-particle matrix elements, here we extend the analysis to the particle-hole channel, deriving the contribution to the continuity equation of the isoscalar and isovector SE, VC and MEC particle-hole matrix elements. We start by evaluating the divergence of the correlation particle-hole matrix element $j_{\text {cor }}^{\mu}(\mathbf{p}, \mathbf{h})$ for the SE and VC contributions; next we address the MEC $\mathrm{p}-\mathrm{h}$ matrix elements.

\section{B.1. Self energy (SE)}

From Eqs. (31) and (32) we get

$$
Q \cdot \mathscr{H}_{p}=-\frac{3 f^{2}}{2 m V m_{\pi}^{2}} \sum_{\mathbf{k} \leqslant k_{\mathrm{F}}} \frac{m}{E_{\mathbf{k}}} \bar{u}(\mathbf{p}) \frac{(\not P-\not K)(K X-m)(\not P-\not K)}{(P-K)^{2}-m_{\pi}^{2}} S_{\mathrm{F}}(P) F_{1} \not P u(\mathbf{h}),
$$




$$
Q \cdot \mathscr{H}_{h}=-\frac{3 f^{2}}{2 m V m_{\pi}^{2}} \sum_{\mathbf{k} \leqslant k_{\mathrm{F}}} \frac{m}{E_{\mathbf{k}}} \bar{u}(\mathbf{p}) F_{1} \emptyset S_{\mathrm{F}}(H) \frac{(K-H I)(K-m)(K-H)}{(K-H)^{2}-m_{\pi}^{2}} u(\mathbf{h}) .
$$

Note that $F_{1}$ cannot be taken out of the matrix element, since it acts on the isospinors. Now from the relations

$$
\begin{aligned}
& S_{\mathrm{F}}(P) Q u(\mathbf{h})=u(\mathbf{h}), \\
& \bar{u}(\mathbf{p}) \Phi S_{\mathrm{F}}(H)=-\bar{u}(\mathbf{p}), \\
& \bar{u}(\mathbf{p})(P-K)(K-m)=2 m \bar{u}(\mathbf{p})(P-K), \\
& (K-m)(K-H) u(\mathbf{h})=-2 m(K-m) u(\mathbf{h}),
\end{aligned}
$$

the following expressions are derived:

$$
\begin{aligned}
& Q \cdot \mathscr{H}_{p}=-\frac{3 f^{2}}{V m_{\pi}^{2}} \sum_{\mathbf{k} \leqslant k_{\mathrm{F}}} \frac{m}{E_{\mathbf{k}}} \bar{u}(\mathbf{p}) \frac{(K-m)(P-K)}{(P-K)^{2}-m_{\pi}^{2}} F_{1} u(\mathbf{h}), \\
& Q \cdot \mathscr{H}_{h}=-\frac{3 f^{2}}{V m_{\pi}^{2}} \sum_{\mathbf{k} \leqslant k_{\mathrm{F}}} \frac{m}{E_{\mathbf{k}}} \bar{u}(\mathbf{p}) F_{1} \frac{(K-H)(K-m)}{(K-H)^{2}-m_{\pi}^{2}} u(\mathbf{h}) .
\end{aligned}
$$

\section{B.2. Vertex correlations ( $V C)$}

From Eqs. (29) and (30) the four-divergence of the VC matrix element is found to be

$$
\begin{aligned}
Q \cdot \mathscr{F} & =-\frac{f^{2}}{V m_{\pi}^{2}} \sum_{\mathbf{k} \leqslant k_{\mathrm{F}}} \frac{m}{E_{\mathbf{k}}} \bar{u}(\mathbf{p}) \gamma_{5}(K-H I) S_{\mathrm{F}}(K+Q) \tau_{a} F_{1} \tau_{a} \Phi \gamma_{5} \frac{\not K-m}{(K-H)^{2}-m_{\pi}^{2}} u(\mathbf{h}), \\
Q \cdot \mathscr{B} & =-\frac{f^{2}}{V m_{\pi}^{2}} \sum_{\mathbf{k} \leqslant k_{\mathrm{F}}} \frac{m}{E_{\mathbf{k}}} \bar{u}(\mathbf{p}) \frac{K-m}{(P-K)^{2}-m_{\pi}^{2}} \tau_{a} F_{1} \tau_{a} \gamma_{5} Q S_{\mathrm{F}}(K-Q) \gamma_{5}(P-K) u(\mathbf{h}) .
\end{aligned}
$$

We now exploit the identities

$$
\begin{aligned}
& S_{\mathrm{F}}(K+Q) Q(K+m)=+(K+m), \\
& (K+m) Q S_{\mathrm{F}}(K-Q)=-(K+m)
\end{aligned}
$$

to get finally

$$
\begin{aligned}
& Q \cdot \mathscr{F}=\frac{f^{2}}{V m_{\pi}^{2}} \sum_{\mathbf{k} \leqslant k_{\mathrm{F}}} \frac{m}{E_{\mathbf{k}}} \bar{u}(\mathbf{p}) \tau_{a} F_{1} \tau_{a} \frac{(K-H I)(K-m)}{(K-H)^{2}-m_{\pi}^{2}} u(\mathbf{h}), \\
& Q \cdot \mathscr{B}=\frac{f^{2}}{V m_{\pi}^{2}} \sum_{\mathbf{k} \leqslant k_{\mathrm{F}}} \frac{m}{E_{\mathbf{k}}} \bar{u}(\mathbf{p}) \tau_{a} F_{1} \tau_{a} \frac{(K-m)(P-K)}{(P-K)^{2}-m_{\pi}^{2}} u(\mathbf{h}) .
\end{aligned}
$$


If expressions (B.7) (B.8), (B.13) and (B.14) are split into their isoscalar and isovector parts, as illustrated in Section 2.2.2, we get

$$
\begin{aligned}
& Q \cdot \mathscr{H}_{p}^{(\mathrm{S})}=-\frac{3 f^{2}}{V m_{\pi}^{2}} F_{1}^{\mathrm{S}} \sum_{\mathbf{k} \leqslant k_{\mathrm{F}}} \frac{m}{E_{\mathbf{k}}} \bar{u}(\mathbf{p}) \frac{(K-m)(P-K)}{(P-K)^{2}-m_{\pi}^{2}} u(\mathbf{h}), \\
& Q \cdot \mathscr{H}_{p}^{(\mathrm{V})}=-\frac{3 f^{2}}{V m_{\pi}^{2}} F_{1}^{\mathrm{V}} \sum_{\mathbf{k} \leqslant k_{\mathrm{F}}} \frac{m}{E_{\mathbf{k}}} \bar{u}(\mathbf{p}) \frac{(K-m)(P-K) \tau_{3}}{(P-K)^{2}-m_{\pi}^{2}} u(\mathbf{h}), \\
& Q \cdot \mathscr{H}_{h}^{(\mathrm{S})}=-\frac{3 f^{2}}{V m_{\pi}^{2}} F_{1}^{\mathrm{S}} \sum_{\mathbf{k} \leqslant k_{\mathrm{F}}} \frac{m}{E_{\mathbf{k}}} \bar{u}(\mathbf{p}) \frac{(K-H I)(K-m)}{(K-H)^{2}-m_{\pi}^{2}} u(\mathbf{h}), \\
& Q \cdot \mathscr{H}_{h}^{(\mathrm{V})}=-\frac{3 f^{2}}{V m_{\pi}^{2}} F_{1}^{\mathrm{V}} \sum_{\mathbf{k} \leqslant k_{\mathrm{F}}} \frac{m}{E_{\mathbf{k}}} \bar{u}(\mathbf{p}) \frac{(K-H)(K K-m) \tau_{3}}{(K-H)^{2}-m_{\pi}^{2}} u(\mathbf{h}), \\
& Q \cdot \mathscr{F}^{(\mathrm{S})}=+\frac{3 f^{2}}{V m_{\pi}^{2}} F_{1}^{\mathrm{S}} \sum_{\mathbf{k} \leqslant k_{\mathrm{F}}} \frac{m}{E_{\mathbf{k}}} \bar{u}(\mathbf{p}) \frac{(K-H I)(K-m)}{(K-H)^{2}-m_{\pi}^{2}} u(\mathbf{h}), \\
& Q \cdot \mathscr{F}^{(\mathrm{V})}=+\frac{f^{2}}{V m_{\pi}^{2}} F_{1}^{\mathrm{V}} \sum_{\mathbf{k} \leqslant k_{\mathrm{F}}} \frac{m}{E_{\mathbf{k}}} \bar{u}(\mathbf{p}) \frac{(K-H H)(K-m)}{(K-H)^{2}-m_{\pi}^{2}}\left(\tau_{3}+\mathrm{i} \varepsilon_{3 a b} \tau_{a} \tau_{b}\right) u(\mathbf{h}), \\
& Q \cdot \mathscr{B}^{(\mathrm{S})}=+\frac{3 f^{2}}{V m_{\pi}^{2}} F_{1}^{\mathrm{S}} \sum_{\mathbf{k} \leqslant k_{\mathrm{F}}} \frac{m}{E_{\mathbf{k}}} \bar{u}(\mathbf{p}) \frac{(K-m)(P-K)}{(P-K)^{2}-m_{\pi}^{2}} u(\mathbf{h}), \\
& Q \cdot \mathscr{B}^{(\mathrm{V})}=+\frac{f^{2}}{V m_{\pi}^{2}} F_{1}^{\mathrm{V}} \sum_{\mathbf{k} \leqslant k_{\mathrm{F}}} \frac{m}{E_{\mathbf{k}}} \bar{u}(\mathbf{p}) \frac{(K-m)(P-\not K)}{(P-K)^{2}-m_{\pi}^{2}}\left(\tau_{3}+\mathrm{i} \varepsilon_{3 a b} \tau_{a} \tau_{b}\right) u(\mathbf{h}) .
\end{aligned}
$$

From these relations we learn that:

- In the isoscalar channel the self-energy and vertex contributions cancel

$$
Q \cdot \mathscr{H}_{p}^{(\mathrm{S})}+Q \cdot \mathscr{B}^{(\mathrm{S})}=Q \cdot \mathscr{H}_{h}^{(\mathrm{S})}+Q \cdot \mathscr{F}^{(\mathrm{S})}=0 .
$$

This differs from the non-relativistic result [24], where the self-energy is by itself gauge invariant. - In the isovector channel we get

$$
\begin{aligned}
& Q \cdot\left[\mathscr{H}_{p}^{(\mathrm{V})}+\mathscr{B}^{(\mathrm{V})}\right]=\frac{2 f^{2}}{V m_{\pi}^{2}} F_{1}^{\mathrm{V}} \mathrm{i} \varepsilon_{3 a b} \sum_{\mathbf{k} \leqslant k_{\mathrm{F}}} \frac{m}{E_{\mathbf{k}}} \bar{u}(\mathbf{p}) \frac{(K-m)(P-K) \tau_{a} \tau_{b}}{(P-K)^{2}-m_{\pi}^{2}}, u(\mathbf{h}) \\
& Q \cdot\left[\mathscr{H}_{h}^{(\mathrm{V})}+\mathscr{F}^{(\mathrm{V})}\right]=\frac{2 f^{2}}{V m_{\pi}^{2}} F_{1}^{\mathrm{V}} \mathrm{i} \varepsilon_{3 a b} \sum_{\mathbf{k} \leqslant k_{\mathrm{F}}} \frac{m}{E_{\mathbf{k}}} \bar{u}(\mathbf{p}) \frac{(K K-H I)(K K-m) \tau_{a} \tau_{b}}{(K-H)^{2}-m_{\pi}^{2}} u(\mathbf{h}) .
\end{aligned}
$$


These expressions, using the Dirac equations $\not h u(\mathbf{h})=m u(\mathbf{h})$ and $\bar{u}(\mathbf{p}) P=m \bar{u}(\mathbf{p})$, can be further simplified to yield the following four-divergence of the correlation current:

$$
\begin{aligned}
Q \cdot j_{\text {cor }}(\mathbf{p}, \mathbf{h}) & =\frac{1}{2} Q \cdot\left[\mathscr{H}_{p}^{(\mathrm{V})}+\mathscr{B}^{(\mathrm{V})}+\mathscr{H}_{h}^{(\mathrm{V})}+\mathscr{F}^{(\mathrm{V})}\right] \\
& =\frac{2 f^{2}}{V m_{\pi}^{2}} F_{1}^{\mathrm{V}} i \varepsilon_{3 a b} \sum_{\mathbf{k} \leqslant k_{\mathrm{F}}} \frac{m}{E_{\mathbf{k}}} \bar{u}(\mathbf{p}) \tau_{a}\left\{\frac{K \cdot P-m^{2}}{(P-K)^{2}-m_{\pi}^{2}}-\frac{K \cdot H-m^{2}}{(K-H)^{2}-m_{\pi}^{2}}\right\} \tau_{b} u(\mathbf{h}) .
\end{aligned}
$$

This contribution is exactly canceled by that of the MEC (seagull and pion-in-flight) as we illustrate in what follows.

\section{B.3. $M E C$}

Using expressions (25) and (26) for the $\mathrm{p}-\mathrm{h}$ matrix elements corresponding to the seagull and pion-in-flight currents, the associated four-divergences are found to be

$$
\begin{aligned}
Q & \cdot j_{\mathrm{s}}(\mathbf{p}, \mathbf{h}) \\
& =-\frac{f^{2}}{V m_{\pi}^{2}} F_{1}^{\mathrm{V}} \mathrm{i} \varepsilon_{3 a b} \sum_{\mathbf{k} \leqslant k_{\mathrm{F}}} \frac{m}{E_{\mathbf{k}}} \bar{u}(\mathbf{p}) \tau_{a} \tau_{b}\left\{\frac{(K-m) Q}{(P-K)^{2}-m_{\pi}^{2}}+\frac{Q(K-m)}{(K-H)^{2}-m_{\pi}^{2}}\right\} u(\mathbf{h}), \\
Q & \cdot j_{\mathrm{p}}(\mathbf{p}, \mathbf{h}) \\
& =\frac{2 m f^{2}}{V m_{\pi}^{2}} F_{1}^{\mathrm{V}} \mathrm{i} \varepsilon_{3 a b} \sum_{\mathbf{k} \leqslant k_{\mathrm{F}}} \frac{m}{E_{\mathbf{k}}} \frac{\left(Q^{2}+2 H \cdot Q-2 K \cdot Q\right)}{\left[(P-K)^{2}-m_{\pi}^{2}\right]\left[(K-H)^{2}-m_{\pi}^{2}\right]} \bar{u}(\mathbf{p}) \tau_{a}(K-m) \tau_{b} u(\mathbf{h}) .
\end{aligned}
$$

Exploiting the Dirac equation and after some algebra the above can be recast as follows:

$$
\begin{aligned}
& Q \cdot j_{\mathrm{MEC}}(\mathbf{p}, \mathbf{h})=Q \cdot j_{\mathrm{s}}(\mathbf{p}, \mathbf{h})+Q \cdot j_{\mathrm{p}}(\mathbf{p}, \mathbf{h}) \\
& \quad=-\frac{2 f^{2}}{V m_{\pi}^{2}} F_{1}^{\mathrm{V}} \mathrm{i} \varepsilon_{3 a b} \sum_{\mathbf{k} \leqslant k_{\mathrm{F}}} \frac{m}{E_{\mathbf{k}}} \bar{u}(\mathbf{p}) \tau_{a}\left\{\frac{K \cdot P-m^{2}}{(P-K)^{2}-m_{\pi}^{2}}-\frac{K \cdot H-m^{2}}{(K-H)^{2}-m_{\pi}^{2}}\right\} \tau_{b} u(\mathbf{h}) .
\end{aligned}
$$

We have thus proven that the correlation and MEC $\mathrm{p}-\mathrm{h}$ matrix elements satisfy current conservation, i.e., $Q \cdot j_{\text {cor }}(\mathbf{p}, \mathbf{h})+Q \cdot j_{\mathrm{MEC}}(\mathbf{p}, \mathbf{h})=0$.

\section{Appendix C. Polarization propagator with nucleon self-energy}

Here we evaluate the Feynman diagrams for the polarization propagator with self-energy $(\Sigma)$ insertions in the particle and hole lines, depicted in Fig. $6 \mathrm{~g}$ and $\mathrm{h}$. From the general Feynman rules for the polarization propagator [43] we have

$$
\begin{aligned}
\Pi_{\mathrm{SE}}^{\mu v}(Q)= & -\mathrm{i} \int \frac{\mathrm{d} h_{0} \mathrm{~d}^{3} h}{(2 \pi)^{4}} \operatorname{Tr}\left\{\Gamma^{\mu}(Q) S_{0}(H) \Sigma(H) S_{0}(H) \Gamma^{v}(-Q) S_{0}(P)\right. \\
& \left.+\Gamma^{\mu}(Q) S_{0}(H) \Gamma^{v}(-Q) S_{0}(P) \Sigma(P) S_{0}(P)\right\}
\end{aligned}
$$


where $P=H+Q$ and $S_{0}$ is the free relativistic propagator for a nucleon in the nuclear medium in Eq. (51), which can also be written in the equivalent ways:

$$
\begin{aligned}
S_{0}(K) & =(K+m)\left[\frac{1}{K^{2}-m^{2}+\mathrm{i} \epsilon}+2 \pi \mathrm{i} \theta\left(k_{\mathrm{F}}-k\right) \delta\left(K^{2}-m^{2}\right) \theta\left(k_{0}\right)\right] \\
& =(K+m)\left[\frac{\theta\left(k-k_{\mathrm{F}}\right)}{K^{2}-m^{2}+\mathrm{i} \epsilon}+\frac{\theta\left(k_{\mathrm{F}}-k\right)}{K^{2}-m^{2}-\mathrm{i} \epsilon k_{0}}\right] .
\end{aligned}
$$

The self-energy function $\Sigma$ is given by Eq. (40).

In order to simplify the calculation of the above polarization propagator, we will simultaneously compute the two diagrams contributing to Eq. (C.1). First we note that Eq. (C.1) can be rewritten as

$$
\Pi_{\mathrm{SE}}^{\mu v}(Q)=\Pi_{10}^{\mu v}(Q)+\Pi_{01}^{\mu v}(Q)
$$

where we introduce $\Pi_{n l}^{\mu v}(Q)$ as the polarization propagator shown in Fig. 34, containing $n$ self-energy insertions $\Sigma(H)$ in the hole line and $l$ insertions $\Sigma(P)$ in the particle line, i.e.,

$$
\Pi_{n l}^{\mu v}(Q) \equiv-\mathrm{i} \operatorname{Tr} \int \frac{\mathrm{d} h_{0} \mathrm{~d}^{3} h}{(2 \pi)^{4}} \Gamma^{\mu}(Q)\left[S_{0}(H) \Sigma(H)\right]^{n} S_{0}(H) \Gamma^{v}(-Q)\left[S_{0}(P) \Sigma(P)\right]^{l} S_{0}(P),
$$

where again $P=H+Q$. From this expression one can derive, as particular cases, the leading-order response $(n=l=0$, no interaction lines) and the first-order self-energy response (with one interaction line, given by Eq. (C.3)).

Using the nucleon propagator in the medium written in the form of Eq. (C.2), the product of $n+1$ propagators appearing in Eq. (C.4) can be expressed as a derivative of order $n$ according to

$$
\begin{aligned}
{\left[S_{0}(H) \Sigma(H)\right]^{n} S_{0}(H) } \\
=[(H+m) \Sigma(H)]^{n}(H+m)\left[\frac{\theta\left(h-k_{\mathrm{F}}\right)}{\left(H^{2}-m^{2}+\mathrm{i} \epsilon\right)^{n+1}}+\frac{\theta\left(k_{\mathrm{F}}-h\right)}{\left(H^{2}-m^{2}-\mathrm{i} \epsilon h_{0}\right)^{n+1}}\right] \\
=\left.[(H+m) \Sigma(H)]^{n}(H+m) \frac{1}{n !} \frac{\mathrm{d}^{n}}{\mathrm{~d} \alpha^{n}}\right|_{\alpha=0}\left[\frac{\theta\left(h-k_{\mathrm{F}}\right)}{H^{2}-\alpha-m^{2}+\mathrm{i} \epsilon}+\frac{\theta\left(k_{\mathrm{F}}-h\right)}{H^{2}-\alpha-m^{2}-\mathrm{i} \epsilon h_{0}}\right] \\
=[(H+m) \Sigma(H)]^{n}(H+m) \\
\times\left.\frac{1}{n !} \frac{\mathrm{d}^{n}}{\mathrm{~d} \alpha^{n}}\right|_{\alpha=0}\left[\frac{1}{H^{2}-\alpha-m^{2}+\mathrm{i} \varepsilon}+2 \pi \mathrm{i} \theta\left(k_{\mathrm{F}}-h\right) \delta\left(H^{2}-\alpha-m^{2}\right) \theta\left(h_{0}\right)\right],
\end{aligned}
$$

where a parameter $\alpha$, which at the end is going to be zero, has been introduced in the propagator denominators. A similar equation holds for the propagation of a particle introducing a second 


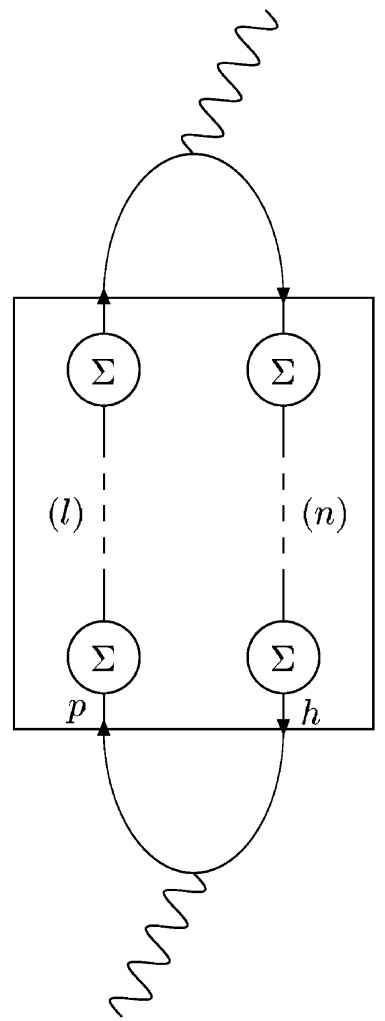

(a)

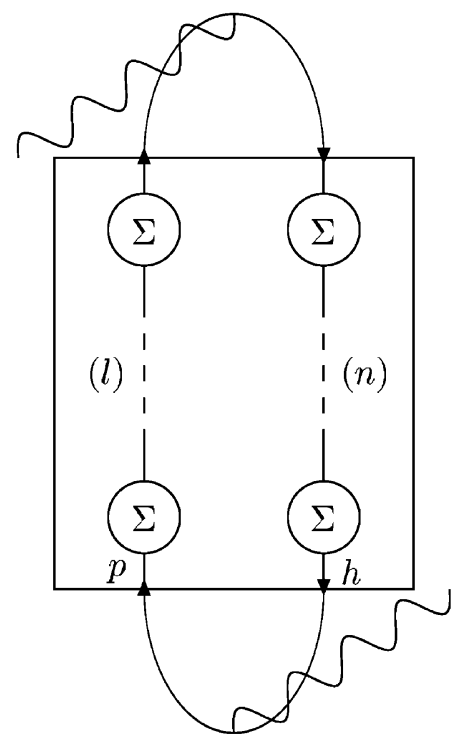

(b)

Fig. 34. Diagrammatic definition of the polarization propagator $\Pi_{n l}^{\mu v}$ for a $p h$ excitation with self-energy insertions in the hole and particle lines. Only the forward diagram (a) contributes to the electromagnetic responses, while the backward diagram (b) corresponds to a negative value of the energy transfer.

parameter $\beta$. The polarization propagator $\Pi_{n l}^{\mu \nu}$ can then be written as

$$
\begin{aligned}
\Pi_{n l}^{\mu v}(Q)= & -\left.\left.\mathrm{i} \frac{\mathrm{d}^{n}}{\mathrm{~d} \alpha^{n}}\right|_{\alpha=0} \frac{\mathrm{d}^{l}}{\mathrm{~d} \beta^{l}}\right|_{\beta=0} \int \frac{\mathrm{d} h_{0} \mathrm{~d}^{3} h}{(2 \pi)^{4}} I_{n l}^{\mu v}(H, P, Q) \\
& \times\left[\frac{1}{H^{2}-\alpha-m^{2}+\mathrm{i} \epsilon}+2 \pi \mathrm{i} \theta\left(k_{\mathrm{F}}-h\right) \delta\left(H^{2}-\alpha-m^{2}\right) \theta\left(h_{0}\right)\right] \\
& \times\left[\frac{1}{P^{2}-\beta-m^{2}+\mathrm{i} \epsilon}+2 \pi \mathrm{i} \theta\left(k_{\mathrm{F}}-p\right) \delta\left(P^{2}-\beta-m^{2}\right) \theta\left(p_{0}\right)\right]
\end{aligned}
$$

with $P=H+Q$, and where we have introduced the functions

$$
\begin{aligned}
I_{n l}^{\mu v}(H, P, Q) & =I_{n l}^{\mu v}\left(h_{0}, \mathbf{h} ; p_{0}, \mathbf{p} ; q_{0}, \mathbf{q}\right) \\
& \equiv \frac{1}{n ! l !} \operatorname{Tr}\left\{\Gamma^{\mu}(Q)[(H+m) \Sigma(H)]^{n}(H+m) \Gamma^{v}(-Q)[(P+m) \Sigma(P)]^{l}(P+m)\right\} .
\end{aligned}
$$


The product of the two brackets inside the integral in Eq. (C.6) gives rise to four terms. The first of these contains the product of the two free propagators, namely

$$
\frac{1}{H^{2}-\alpha-m^{2}+\mathrm{i} \epsilon} \times \frac{1}{P^{2}-\beta-m^{2}+\mathrm{i} \epsilon},
$$

and yields a genuine vacuum contribution, $\Pi_{n l}^{(0) \mu v}(Q)$, which diverges after integration. Therefore we subtract out its contribution since it pertains to a domain beyond nuclear physics. Performing this subtraction of the vacuum propagator we obtain

$$
\begin{aligned}
\Pi_{n l}^{\mu v}(Q)-\Pi_{n l}^{(0) \mu v}(Q)= & \left.\left.2 \pi \frac{\mathrm{d}^{n}}{\mathrm{~d} \alpha^{n}}\right|_{\alpha=0} \frac{\mathrm{d}^{l}}{\mathrm{~d} \beta^{l}}\right|_{\beta=0} \int \frac{\mathrm{d} h_{0} \mathrm{~d}^{3} h}{(2 \pi)^{4}} I_{n l}^{\mu v}(H, P, Q) \\
& \times\left[\frac{\theta\left(k_{\mathrm{F}}-p\right) \delta\left(P^{2}-\beta-m^{2}\right) \theta\left(p_{0}\right)}{H^{2}-\alpha-m^{2}+\mathrm{i} \epsilon}+\frac{\theta\left(k_{\mathrm{F}}-h\right) \delta\left(H^{2}-\alpha-m^{2}\right) \theta\left(h_{0}\right)}{H^{2}-\beta-m^{2}+\mathrm{i} \epsilon}\right. \\
& \left.+2 \pi \mathrm{i} \theta\left(k_{\mathrm{F}}-p\right) \theta\left(k_{\mathrm{F}}-h\right) \delta\left(P^{2}-\beta-m^{2}\right) \delta\left(H^{2}-\alpha-m^{2}\right) \theta\left(P_{0}\right) \theta\left(H_{0}\right)\right] .
\end{aligned}
$$

Taking the imaginary part according to Eq. (11) we obtain the corresponding hadronic tensor ${ }^{6}$

$$
\begin{aligned}
-\frac{V}{\pi} & \operatorname{Im}\left[\Pi_{n l}^{\mu v}(Q)-\Pi_{n l}^{(0) \mu v}(Q)\right] \\
= & \left.\left.2 \pi \frac{\mathrm{d}^{n}}{\mathrm{~d} \alpha^{n}}\right|_{\alpha=0} \frac{\mathrm{d}^{l}}{\mathrm{~d} \beta^{l}}\right|_{\beta=0} \int \frac{\mathrm{d} h_{0} \mathrm{~d}^{3} h}{(2 \pi)^{4}} I_{n l}^{\mu v}(H, P, Q) \delta\left(P^{2}-\beta-m^{2}\right) \delta\left(H^{2}-\alpha-m^{2}\right) \\
& \times\left[\theta\left(k_{\mathrm{F}}-p\right) \theta\left(p_{0}\right)+\theta\left(k_{\mathrm{F}}-h\right) \theta\left(h_{0}\right)-2 \theta\left(k_{\mathrm{F}}-p\right) \theta\left(k_{\mathrm{F}}-h\right) \theta\left(p_{0}\right) \theta\left(h_{0}\right)\right] .
\end{aligned}
$$

Now the factor containing the step functions can be expressed in the form

$$
\begin{aligned}
& {\left[\theta\left(k_{\mathrm{F}}-p\right) \theta\left(p_{0}\right)+\theta\left(k_{\mathrm{F}}-h\right) \theta\left(h_{0}\right)-2 \theta\left(k_{\mathrm{F}}-p\right) \theta\left(k_{\mathrm{F}}-h\right) \theta\left(p_{0}\right) \theta\left(h_{0}\right)\right]} \\
& \quad=\theta\left(k_{\mathrm{F}}-h\right) \theta\left(h_{0}\right)\left[1-\theta\left(k_{\mathrm{F}}-p\right) \theta\left(p_{0}\right)\right]+\theta\left(k_{\mathrm{F}}-p\right) \theta\left(p_{0}\right)\left[1-\theta\left(k_{\mathrm{F}}-h\right) \theta\left(h_{0}\right)\right]
\end{aligned}
$$

so that the hadronic tensor can be written as a sum of two pieces

$$
-\frac{V}{\pi} \operatorname{Im}\left[\Pi_{n l}^{\mu v}-\Pi_{n l}^{(0) \mu v}\right]=W_{n l}^{(+) \mu v}+W_{n l}^{(-) \mu v}
$$

where

$$
\begin{aligned}
W_{n l}^{(+) \mu v}(Q)= & \left.\left.2 \pi V \frac{\mathrm{d}^{n}}{\mathrm{~d} \alpha^{n}}\right|_{\alpha=0} \frac{\mathrm{d}^{l}}{\mathrm{~d} \beta^{l}}\right|_{\beta=0} \int \frac{\mathrm{d} h_{0} \mathrm{~d}^{3} h}{(2 \pi)^{4}} I_{n l}^{\mu v}(H, P, Q) \delta\left(P^{2}-\beta-m^{2}\right) \delta\left(H^{2}-\alpha-m^{2}\right) \\
& \times \theta\left(k_{\mathrm{F}}-h\right) \theta\left(h_{0}\right)\left[1-\theta\left(k_{\mathrm{F}}-p\right) \theta\left(p_{0}\right)\right]
\end{aligned}
$$

\footnotetext{
${ }^{6}$ The extra factor $V$ appears since we are computing the response function of an extended system, see Eq. (17.17) of Ref. [43]
} 
corresponds to the hadronic tensor sought for electron scattering (Fig. 34a), whereas the second term

$$
\begin{aligned}
W_{n l}^{(-) \mu v}(Q)= & \left.\left.2 \pi V \frac{\mathrm{d}^{n}}{\mathrm{~d} \alpha^{n}}\right|_{\alpha=0} \frac{\mathrm{d}^{l}}{\mathrm{~d} \beta^{l}}\right|_{\beta=0} \int \frac{\mathrm{d} h_{0} \mathrm{~d}^{3} h}{(2 \pi)^{4}} I_{n l}^{\mu v}(H, P, Q) \delta\left(P^{2}-\beta-m^{2}\right) \delta\left(H^{2}-\alpha-m^{2}\right) \\
& \times \theta\left(k_{\mathrm{F}}-p\right) \theta\left(p_{0}\right)\left[1-\theta\left(k_{\mathrm{F}}-h\right) \theta\left(h_{0}\right)\right]
\end{aligned}
$$

corresponds to a process with negative energy transfer (Fig. 34b); hence it does not contribute to the electron scattering response and should be disregarded.

Finally, the integration with respect to $h_{0}$ in Eq. (C.13) can be explicitly performed by using the $\delta$-functions. One then gets the following expression for the $n l$ th $\mathrm{SE}$ contribution to the hadronic tensor:

$$
\begin{aligned}
W_{n l}^{\mu v} \equiv W_{n l}^{(+) \mu v}= & \left.\left.V \frac{\mathrm{d}^{n}}{\mathrm{~d} \alpha^{n}}\right|_{0} \frac{\mathrm{d}^{l}}{\mathrm{~d} \beta^{l}}\right|_{0} \int \frac{\mathrm{d}^{3} h}{(2 \pi)^{3}} \frac{I_{n l}^{\mu v}\left(E_{\mathbf{h}}^{\prime}(\alpha), \mathbf{h} ; E_{\mathbf{p}}^{\prime}(\beta), \mathbf{p} ; q\right)}{4 E_{\mathbf{h}}^{\prime}(\alpha) E_{\mathbf{p}}^{\prime}(\beta)} \\
& \times \delta\left(E_{\mathbf{h}}^{\prime}(\alpha)+q_{0}-E_{\mathbf{p}}^{\prime}(\beta)\right) \theta\left(k_{\mathrm{F}}-h\right) \theta\left(p-k_{\mathrm{F}}\right),
\end{aligned}
$$

where $\mathbf{p}=\mathbf{h}+\mathbf{q}$ and we have defined the following energy functions of the parameters $\alpha, \beta$ :

$$
\begin{aligned}
& E_{\mathbf{h}}^{\prime}(\alpha)=\sqrt{\mathbf{h}^{2}+\alpha+m^{2}}, \\
& E_{\mathbf{p}}^{\prime}(\beta)=\sqrt{\mathbf{p}^{2}+\beta+m^{2}} .
\end{aligned}
$$

Expression (C.15) is the general equation for which we are searching. It is one of the $(n+l)$ th order contributions to the full HF hadronic tensor, which is an infinite sum of all perturbative orders. In the particular case $n=l=0$ it gives the well known free (OB) hadronic tensor

$$
W_{\mathrm{OB}}^{\mu v}=W_{00}^{\mu v}=V \int \frac{\mathrm{d}^{3} h}{(2 \pi)^{3}} \frac{I_{00}^{\mu v}\left(E_{\mathbf{h}}, \mathbf{h} ; E_{\mathbf{p}}, \mathbf{p} ; q\right)}{4 E_{\mathbf{h}} E_{\mathbf{p}}} \delta\left(E_{\mathbf{h}}+q_{0}-E_{\mathbf{p}}\right) \theta\left(k_{\mathrm{F}}-h\right) \theta\left(p-k_{\mathrm{F}}\right) .
$$

Finally, the hadronic tensor corresponding to one self-energy insertion in the particle or hole lines, corresponding to diagrams $(\mathrm{g})$ and $(\mathrm{h})$ in Fig. 6 is given by

$$
W_{\mathrm{SE}}^{\mu v}=W_{10}^{\mu v}+W_{01}^{\mu v}
$$

where the $n=1, l=0$ terms correspond to the first-order hole self-energy diagram (Fig. $6 \mathrm{~h}$ )

$$
W_{10}^{\mu v}=\left.V \frac{\mathrm{d}}{\mathrm{d} \alpha}\right|_{\alpha=0} \int \frac{\mathrm{d}^{3} h}{(2 \pi)^{3}} \frac{I_{10}^{\mu v}\left(E_{\mathbf{h}}^{\prime}(\alpha), \mathbf{h} ; E_{\mathbf{p}}, \mathbf{p} ; q\right)}{4 E_{\mathbf{h}}^{\prime}(\alpha) E_{\mathbf{p}}} \delta\left(E_{\mathbf{h}}^{\prime}(\alpha)+q_{0}-E_{\mathbf{p}}\right) \theta\left(k_{\mathrm{F}}-h\right) \theta\left(p-k_{\mathrm{F}}\right)
$$

and for $n=0, l=1$, the first-order particle self-energy diagram (Fig. 6g)

$$
W_{01}^{\mu v}=\left.V \frac{\mathrm{d}}{\mathrm{d} \beta}\right|_{\beta=0} \int \frac{\mathrm{d}^{3} h}{(2 \pi)^{3}} \frac{I_{01}^{\mu v}\left(E_{\mathbf{h}}, \mathbf{h} ; E_{\mathbf{p}}^{\prime}(\beta), \mathbf{p} ; q\right)}{4 E_{\mathbf{h}} E_{\mathbf{p}}^{\prime}(\beta)} \delta\left(E_{\mathbf{h}}+q_{0}-E_{\mathbf{p}}^{\prime}(\beta)\right) \theta\left(k_{\mathrm{F}}-h\right) \theta\left(p-k_{\mathrm{F}}\right) .
$$


In the above expressions, after the derivatives with respect to the parameters $\alpha$ and $\beta$ are taken, the integral over the hole polar angle $\cos \theta_{h}$ can be performed analytically by exploiting the $\delta$-function. Hence the SE contribution to the hadronic tensor can finally be expressed as a double integral. Since the self-energy $\Sigma$ involves a triple integral, the contribution to hadronic tensor turns out to be a five-dimensional integral, to be carried out numerically.

\section{Appendix D. Renormalized self-energy response using the polarization propagator}

In Appendix $\mathrm{C}$ we computed the first-order self-energy contribution to the polarization propagator corresponding to the two diagrams of Fig. 6. The corresponding hadronic tensor splits into the sum of the two terms given in Eqs. (C.20) and (C.21) with Fock self-energy insertions in the hole and particle lines respectively, and reads

$$
\begin{aligned}
W^{\mu v}= & \left.V \frac{\mathrm{d}}{\mathrm{d} \alpha}\right|_{\alpha=0} \int \frac{\mathrm{d}^{3} h}{(2 \pi)^{3}} \frac{I_{10}^{\mu v}\left(E_{\mathbf{h}}^{\prime}(\alpha), \mathbf{h} ; E_{\mathbf{p}}, \mathbf{p} ; q\right)}{4 E_{\mathbf{h}}^{\prime}(\alpha) E_{\mathbf{p}}} \delta\left(E_{\mathbf{h}}^{\prime}(\alpha)+q_{0}-E_{\mathbf{p}}\right) \theta\left(k_{\mathrm{F}}-h\right) \theta\left(p-k_{\mathrm{F}}\right) \\
& +\left.V \frac{\mathrm{d}}{\mathrm{d} \beta}\right|_{\beta=0} \int \frac{\mathrm{d}^{3} h}{(2 \pi)^{3}} \frac{I_{01}^{\mu v}\left(E_{\mathbf{h}}, \mathbf{h} ; E_{\mathbf{p}}^{\prime}(\beta), \mathbf{p} ; q\right)}{4 E_{\mathbf{h}} E_{\mathbf{p}}^{\prime}(\beta)} \delta\left(E_{\mathbf{h}}+q_{0}-E_{\mathbf{p}}^{\prime}(\beta)\right) \theta\left(k_{\mathrm{F}}-h\right) \theta\left(p-k_{\mathrm{F}}\right),
\end{aligned}
$$

where $\mathbf{p}=\mathbf{h}+\mathbf{q}$, and the modified energies for holes and particles have been introduced in Eqs. (C.16) and (C.17), with $\alpha$ and $\beta$ being real parameters. Finally, the functions $I_{n l}^{\mu v}$ are defined in Eq. (C.7).

In order to prove the equivalence between the responses computed using the polarization propagator in Eq. (D.1) and the result in Eq. (145), obtained using the renormalized current and energies, we proceed to perform the derivative with respect to $\alpha$ and $\beta$. For a general function $F\left(h_{0}\right)$ we have

$$
\left.\frac{\mathrm{d} F\left(E_{\mathbf{h}}^{\prime}(\alpha)\right)}{\mathrm{d} \alpha}\right|_{\alpha=0}=\frac{1}{2 E_{\mathbf{h}}}\left[\frac{\mathrm{d} F\left(h_{0}\right)}{\mathrm{d} h_{0}}\right]_{h_{0}=E_{\mathbf{h}}} .
$$

Hence, interchanging the derivatives and the integral, we get for the hadronic tensor the expression

$$
\begin{aligned}
W^{\mu v}= & V \int \frac{\mathrm{d}^{3} h}{(2 \pi)^{3}} \frac{1}{4 E_{\mathbf{h}} E_{\mathbf{p}}} \frac{\mathrm{d}}{\mathrm{d} h_{0}}\left[\frac{I_{10}^{\mu v}\left(h_{0}, \mathbf{h} ; E_{\mathbf{p}}, \mathbf{p} ; q\right)}{2 h_{0}}\right]_{h_{0}=E_{\mathbf{h}}} \delta\left(E_{\mathbf{h}}+q_{0}-E_{\mathbf{p}}\right) \theta\left(k_{\mathrm{F}}-h\right) \theta\left(p-k_{\mathrm{F}}\right) \\
& +V \int \frac{\mathrm{d}^{3} h}{(2 \pi)^{3}} \frac{1}{4 E_{\mathbf{h}} E_{\mathbf{p}}} I_{10}^{\mu v}\left(E_{\mathbf{h}}, \mathbf{h} ; E_{\mathbf{p}}, \mathbf{p} ; q\right) \frac{1}{2 E_{\mathbf{h}}} \frac{\mathrm{d}}{\mathrm{d} q_{0}} \delta\left(E_{\mathbf{h}}+q_{0}-E_{\mathbf{p}}\right) \theta\left(k_{\mathrm{F}}-h\right) \theta\left(p-k_{\mathrm{F}}\right) \\
& +V \int \frac{\mathrm{d}^{3} h}{(2 \pi)^{3}} \frac{1}{4 E_{\mathbf{h}} E_{\mathbf{p}}} \frac{\mathrm{d}}{\mathrm{d} p_{0}}\left[\frac{I_{01}^{\mu v}\left(E_{\mathbf{h}}, \mathbf{h} ; p_{0}, \mathbf{p} ; q\right)}{2 p_{0}}\right]_{p_{0}=E_{\mathbf{p}}} \delta\left(E_{\mathbf{h}}+q_{0}-E_{\mathbf{p}}\right) \theta\left(k_{\mathrm{F}}-h\right) \theta\left(p-k_{\mathrm{F}}\right) \\
& -V \int \frac{\mathrm{d}^{3} h}{(2 \pi)^{3}} \frac{1}{4 E_{\mathbf{h}} E_{\mathbf{p}}} I_{01}^{\mu v}\left(E_{\mathbf{h}}, \mathbf{h} ; E_{\mathbf{p}}, \mathbf{p} ; q\right) \frac{1}{2 E_{\mathbf{p}}} \frac{\mathrm{d}}{\mathrm{d} q_{0}} \delta\left(E_{\mathbf{h}}+q_{0}-E_{\mathbf{p}}\right) \theta\left(k_{\mathrm{F}}-h\right) \theta\left(p-k_{\mathrm{F}}\right) .
\end{aligned}
$$


In differentiating the function $I_{01}^{\mu v}$ defined in Eq. (C.7), we first consider the term

$$
\begin{aligned}
\frac{\mathrm{d}}{\mathrm{d} p_{0}} & {\left[\frac{1}{2 p_{0}}(P+m) \Sigma(P)(P+m)\right]_{p_{0}=E_{\mathbf{p}}} } \\
= & \frac{\sum_{0}(\mathbf{p})}{2 E_{\mathbf{p}}}\left[-\frac{2 m}{E_{\mathbf{p}}}(P+m)+\gamma_{0}(P+m)+(P+m) \gamma_{0}\right]_{p_{0}=E_{\mathbf{p}}} \\
& +\left[\frac{1}{2 E_{\mathbf{h}}}(H+m) \frac{\partial \Sigma(H)}{\partial p_{0}}(H+m)\right]_{p_{0}=E_{\mathbf{p}}},
\end{aligned}
$$

where use has been made of the results

$$
\begin{aligned}
& \Sigma(P)(\not P+m)=\Sigma_{0}(\mathbf{p})(\not P+m), \\
& (\not P+m)(\not P+m)=2 m(\not P+m),
\end{aligned}
$$

which hold for $P^{\mu}$ on-shell and where $\Sigma_{0}(\mathbf{p})$ is the eigenvalue of the self-energy for on-shell spinors.

Next we should compute the derivative of the self-energy $\Sigma(P)$. This function has the general structure given in Eq. (44), and its derivative implies derivatives of the coefficients $A, B$ and $C$, namely

$$
\frac{\partial \Sigma(P)}{\partial p_{0}}=m \frac{\partial A(P)}{\partial p_{0}}+\frac{\partial B(P)}{\partial p_{0}} \gamma_{0} p_{0}-\frac{\partial C(P)}{\partial p_{0}} \boldsymbol{\gamma} \cdot \mathbf{p}+B(P) \gamma_{0},
$$

which must be evaluated for $P^{\mu}$ on-shell. Using again Eq. (D.6) together with the identity

$$
(P+m) \gamma^{\mu}(P+m)=2 P^{\mu}(P+m)
$$

we obtain, for $P$ on-shell,

$$
\begin{aligned}
& \frac{1}{2 E_{\mathbf{p}}}(\not P+m) \frac{\partial \Sigma(P)}{\partial p_{0}}(\not P+m) \\
& \quad=\frac{1}{E_{\mathbf{p}}}\left[m^{2} \frac{\partial A(P)}{\partial p_{0}} E_{\mathbf{p}}^{2} \frac{\partial B(P)}{\partial p_{0}}-\mathbf{p}^{2} \frac{\partial C(P)}{\partial p_{0}}+E_{\mathbf{p}} B_{0}(\mathbf{p})\right]_{p_{0}=E_{\mathbf{p}}}(P+m) \\
& \quad=\alpha(\mathbf{p})(P+m)
\end{aligned}
$$

where the definition of the function $\alpha(\mathbf{p})$ in Eq. (100) has been used.

Finally, collecting the above results, the derivative in Eq. (D.4) is found to read

$$
\begin{aligned}
\frac{\mathrm{d}}{\mathrm{d} p_{0}} & {\left[\frac{1}{2 p_{0}}(P+m) \Sigma(P)(P+m)\right]_{p_{0}=E_{\mathbf{p}}} } \\
= & \frac{\sum_{0}(\mathbf{p})}{E_{\mathbf{p}}}\left[\frac{\gamma_{0} E_{\mathbf{p}}-m}{2 E_{\mathbf{p}}}(\not P+m)+(\not P+m) \frac{\gamma_{0} E_{\mathbf{p}}-m}{2 E_{\mathbf{p}}}\right]+\alpha(\mathbf{p})(P+m) .
\end{aligned}
$$


Hence the following expression

$$
\begin{aligned}
\frac{\mathrm{d}}{\mathrm{d} p_{0}} & {\left[\frac{I_{01}^{\mu v}(H, P, Q)}{2 p_{0}}\right]_{p_{0}=E_{\mathbf{p}}} } \\
= & \operatorname{Tr}\left\{\Gamma^{\mu}(Q)(H+m) \Gamma^{v}(-Q)\left[\frac{\Sigma_{0}(\mathbf{p})}{E_{\mathbf{p}}} \frac{\gamma_{0} E_{\mathbf{p}}-m}{2 E_{\mathbf{p}}}+\frac{\alpha(\mathbf{p})}{2}\right](P+m)\right\} \\
& +\operatorname{Tr}\left\{\Gamma^{\mu}(Q)(H+m) \Gamma^{v}(-Q)(P+m)\left[\frac{\Sigma_{0}(\mathbf{p})}{E_{\mathbf{p}}} \frac{\gamma_{0} E_{\mathbf{p}}-m}{2 E_{\mathbf{p}}}+\frac{\alpha(\mathbf{p})}{2}\right]\right\}
\end{aligned}
$$

yields the derivative of $I_{01}^{\mu v}(H, P, Q)$ with respect to $p_{0}$ and the similar result

$$
\begin{aligned}
\frac{\mathrm{d}}{\mathrm{d} h_{0}} & {\left[\frac{I_{10}^{\mu v}(H, P, Q)}{2 h_{0}}\right]_{h_{0}=E_{\mathbf{h}}} } \\
= & \operatorname{Tr}\left\{\Gamma^{\mu}(Q)\left[\frac{\Sigma_{0}(\mathbf{h})}{E_{\mathbf{h}}} \frac{\gamma_{0} E_{\mathbf{h}}-m}{2 E_{\mathbf{h}}}+\frac{\alpha(\mathbf{h})}{2}\right](H+m) \Gamma^{v}(-Q)(P+m)\right\} \\
& +\operatorname{Tr}\left\{\Gamma^{\mu}(Q)(H+m)\left[\frac{\Sigma_{0}(\mathbf{h})}{E_{\mathbf{h}}} \frac{\gamma_{0} E_{\mathbf{h}}-m}{2 E_{\mathbf{h}}}+\frac{\alpha(\mathbf{h})}{2}\right] \Gamma^{v}(-Q)(P+m)\right\}
\end{aligned}
$$

holds for the derivative of $I_{10}^{\mu \nu}(H, P, Q)$ with respect to $h_{0}$. In addition, with the help of Eq. (D.5), we can write for on-shell momenta

$$
\begin{aligned}
I_{10}^{\mu v}(H, P, Q) & =\operatorname{Tr}\left\{\Gamma^{\mu}(Q)(H+m) \Sigma(H)(H+m) \Gamma^{v}(-Q)(P+m)\right\} \\
& =2 m \Sigma_{0}(\mathbf{h}) \operatorname{Tr}\left\{\Gamma^{\mu}(Q)(H+m) \Gamma^{v}(-Q)(P+m)\right\}
\end{aligned}
$$

and, as well,

$$
I_{01}^{\mu v}(H, P, Q)=2 m \Sigma_{0}(\mathbf{p}) \operatorname{Tr}\left\{\Gamma^{\mu}(Q)(H+m) \Gamma^{v}(-Q)(P+m)\right\}
$$

Finally, the response functions are found as linear combinations of the diagonal components of the hadronic tensor, i.e., $W^{\mu \mu}$. Using the above equations the latter reads

$$
\begin{aligned}
W^{\mu \mu}= & V \int \frac{\mathrm{d}^{3} h}{(2 \pi)^{3}} \frac{1}{4 E_{\mathbf{h}} E_{\mathbf{p}}} \delta\left(E_{\mathbf{h}}+q_{0}-E_{\mathbf{p}}\right) \theta\left(k_{\mathrm{F}}-h\right) \theta\left(p-k_{\mathrm{F}}\right) \\
& \times \operatorname{Tr}\left\{\Gamma^{\mu}(Q)\left[\frac{\Sigma_{0}(\mathbf{h})}{E_{\mathbf{h}}} \frac{\gamma_{0} E_{\mathbf{h}}-m}{2 E_{\mathbf{h}}}+\frac{\alpha(\mathbf{h})}{2}\right](H+m) \Gamma^{\mu}(-Q)(P+m)\right. \\
& +\Gamma^{\mu}(Q)(H+m)\left[\frac{\Sigma_{0}(\mathbf{h})}{E_{\mathbf{h}}} \frac{\gamma_{0} E_{\mathbf{h}}-m}{2 E_{\mathbf{h}}}+\frac{\alpha(\mathbf{h})}{2}\right] \Gamma^{\mu}(-Q)(P+m)
\end{aligned}
$$




$$
\begin{aligned}
& +\Gamma^{\mu}(Q)(H+m) \Gamma^{\mu}(-Q)\left[\frac{\Sigma_{0}(\mathbf{p})}{E_{\mathbf{p}}} \frac{\gamma_{0} E_{\mathbf{p}}-m}{2 E_{\mathbf{p}}}+\frac{\alpha(\mathbf{p})}{2}\right](P+m) \\
& \left.+\Gamma^{\mu}(Q)(H+m) \Gamma^{\mu}(-Q)(P+m)\left[\frac{\Sigma_{0}(\mathbf{p})}{E_{\mathbf{p}}} \frac{\gamma_{0} E_{\mathbf{p}}-m}{2 E_{\mathbf{p}}}+\frac{\alpha(\mathbf{p})}{2}\right]\right\} \\
& +V \int \frac{\mathrm{d}^{3} h}{(2 \pi)^{3}} \frac{1}{4 E_{\mathbf{h}} E_{\mathbf{p}}} \operatorname{Tr}\left\{\Gamma^{\mu}(Q)(H+m) \Gamma^{\mu}(-Q)(P+m)\right\} \\
& \times\left(\Sigma_{0}(\mathbf{h}) \frac{m}{E_{\mathbf{h}}}-\Sigma_{0}(\mathbf{p}) \frac{m}{E_{\mathbf{p}}}\right) \frac{\mathrm{d}}{\mathrm{d} q_{0}} \delta\left(E_{\mathbf{h}}+q_{0}-E_{\mathbf{p}}\right) \theta\left(k_{\mathrm{F}}-h\right) \theta\left(p-k_{\mathrm{F}}\right),
\end{aligned}
$$

which coincides with the result in Eq. (145), obtained by computing the response functions using the renormalized current and energy.

\section{Appendix E. On-shell self-energy and field strength renormalization function}

In this appendix we show in detail how to evaluate the on-shell self-energy in Eq. (82) and the field strength renormalization function in Eq. (100). They can be expressed in terms of the integrals $I(P)$ and $L^{\mu}(P)$ in Eqs. (45) and (46) as follows:

$$
\Sigma_{0}(\mathbf{p})=2 m B\left(E_{\mathbf{p}}, \mathbf{p}\right)=-12 m \frac{f^{2}}{m_{\pi}^{2}}\left[p_{0} L_{0}\left(p_{0}, \mathbf{p}\right)-p L_{3}\left(p_{0}, \mathbf{p}\right)-m^{2} I\left(p_{0}, \mathbf{p}\right)\right]_{p_{0}=E_{\mathbf{p}}}
$$

and

$$
\begin{aligned}
\alpha(\mathbf{p}) & =B_{0}(\mathbf{p})+\frac{1}{E_{\mathbf{p}}}\left[m^{2} \frac{\partial A\left(p_{0}, \mathbf{p}\right)}{\partial p_{0}}+E_{\mathbf{p}}^{2} \frac{\partial B\left(p_{0}, \mathbf{p}\right)}{\partial p_{0}}-\mathbf{p}^{2} \frac{\partial C\left(p_{0}, \mathbf{p}\right)}{\partial p_{0}}\right]_{p_{0}=E_{\mathbf{p}}} \\
& =-12 m^{2} \frac{f^{2}}{m_{\pi}^{2}}\left[\frac{L_{0}\left(p_{0}, \mathbf{p}\right)}{p_{0}}-I\left(p_{0}, \mathbf{p}\right)+\frac{\partial L_{0}\left(p_{0}, \mathbf{p}\right)}{\partial p_{0}}-\frac{p}{p_{0}} \frac{\partial L_{3}\left(p_{0}, \mathbf{p}\right)}{\partial p_{0}}-\frac{m^{2}}{p_{0}} \frac{\partial I\left(p_{0}, \mathbf{p}\right)}{\partial p_{0}}\right]_{p_{0}=E_{\mathbf{p}}},
\end{aligned}
$$

where we have used Eqs. (47)-(49) and the derivatives

$$
\begin{aligned}
\left(\frac{\partial A\left(p_{0}, \mathbf{p}\right)}{\partial p_{0}}\right)_{p_{0}=E_{\mathbf{p}}}= & -6 \frac{f^{2}}{m_{\pi}^{2}}\left[p_{0} \frac{\partial L_{0}\left(p_{0}, \mathbf{p}\right)}{\partial p_{0}}-p \frac{\partial L_{3}\left(p_{0}, \mathbf{p}\right)}{\partial p_{0}}-m^{2} \frac{\partial I\left(p_{0}, \mathbf{p}\right)}{\partial p_{0}}\right. \\
& \left.+L_{0}\left(p_{0}, \mathbf{p}\right)-p_{0} I\left(p_{0}, \mathbf{p}\right)\right]_{p_{0}=E_{\mathbf{p}}} \\
\left(\frac{\partial B\left(p_{0}, \mathbf{p}\right)}{\partial p_{0}}\right)_{p_{0}=E_{\mathbf{p}}}= & -6 \frac{f^{2}}{m_{\pi}^{2}}\left[p_{0} \frac{\partial L_{0}\left(p_{0}, \mathbf{p}\right)}{\partial p_{0}}-p \frac{\partial L_{3}\left(p_{0}, \mathbf{p}\right)}{\partial p_{0}}-m^{2} \frac{\partial I\left(p_{0}, \mathbf{p}\right)}{\partial p_{0}}\right]_{p_{0}=E_{\mathbf{p}}}
\end{aligned}
$$




$$
\begin{aligned}
\left(\frac{\partial C\left(p_{0}, \mathbf{p}\right)}{\partial p_{0}}\right)_{p_{0}=E_{\mathbf{p}}}= & -6 \frac{f^{2}}{m_{\pi}^{2}}\left[p_{0} \frac{\partial L_{0}\left(p_{0}, \mathbf{p}\right)}{\partial p_{0}}-p \frac{\partial L_{3}\left(p_{0}, \mathbf{p}\right)}{\partial p_{0}}-m^{2} \frac{\partial I\left(p_{0}, \mathbf{p}\right)}{\partial p_{0}}\right. \\
& \left.+L_{0}\left(p_{0}, \mathbf{p}\right)-\frac{p_{0}}{p} L_{3}\left(p_{0}, \mathbf{p}\right)\right]_{p_{0}=E_{\mathbf{p}}} .
\end{aligned}
$$

By choosing the $z$-axis in the direction of $\mathbf{p}$, the angular integrals can be performed analytically, yielding

$$
\begin{aligned}
& I\left(E_{\mathbf{p}}, \mathbf{p}\right)=\frac{1}{(2 \pi)^{2}} \int_{0}^{k_{\mathrm{F}}} \mathrm{d} k \frac{k}{4 p E_{\mathbf{k}}} \ln \frac{\gamma(p, k)+2 p k}{\gamma(p, k)-2 p k}, \\
& L_{0}\left(E_{\mathbf{p}}, \mathbf{p}\right)=\frac{1}{(2 \pi)^{2}} \int_{0}^{k_{\mathrm{F}}} \mathrm{d} k \frac{k}{4 p} \ln \frac{\gamma(p, k)+2 p k}{\gamma(p, k)-2 p k}, \\
& L_{3}\left(E_{\mathbf{p}}, \mathbf{p}\right)=\frac{1}{(2 \pi)^{2}} \int_{0}^{k_{\mathrm{F}}} \mathrm{d} k\left\{\frac{k^{2}}{2 p E_{\mathbf{k}}}-\frac{k \gamma(p, k)}{8 p^{2} E_{\mathbf{k}}} \ln \frac{\gamma(p, k)+2 p k}{\gamma(p, k)-2 p k}\right\}, \\
& \left.\frac{\partial I\left(p_{0}, \mathbf{p}\right)}{\partial p_{0}}\right|_{p_{0}=E_{\mathbf{p}}}=\frac{1}{(2 \pi)^{2}} \int_{0}^{k_{\mathrm{F}}} \mathrm{d} k \frac{k}{2 p E_{\mathbf{k}}}\left(E_{\mathbf{p}}-E_{\mathbf{k}}\right)\left[\frac{1}{\gamma(p, k)+2 p k}-\frac{1}{\gamma(p, k)-2 p k}\right], \\
& \left.\frac{\partial L_{0}\left(p_{0}, \mathbf{p}\right)}{\partial p_{0}}\right|_{p_{0}=E_{\mathbf{p}}}=\frac{1}{(2 \pi)^{2}} \int_{0}^{k_{\mathrm{F}}} \mathrm{d} k \frac{k}{2 p}\left(E_{\mathbf{p}}-E_{\mathbf{k}}\right)\left[\frac{1}{\gamma(p, k)+2 p k}-\frac{1}{\gamma(p, k)-2 p k}\right], \\
& \left.\frac{\partial L_{3}\left(p_{0}, \mathbf{p}\right)}{\partial p_{0}}\right|_{p_{0}=E_{\mathbf{p}}}=-\frac{1}{(2 \pi)^{2}} \int_{0}^{k_{\mathrm{F}}} \mathrm{d} k \frac{k}{4 p^{2} E_{\mathbf{k}}}\left(E_{\mathbf{p}}-E_{\mathbf{k}}\right)\left\{\ln \frac{\gamma(p, k)+2 p k}{\gamma(p, k)-2 p k}\right. \\
& \left.+\gamma(p, k)\left[\frac{1}{\gamma(p, k)+2 p k}-\frac{1}{\gamma(p, k)-2 p k}\right]\right\} \text {, }
\end{aligned}
$$

where we have defined the function

$$
\gamma(p, k) \equiv\left(E_{\mathbf{p}}-E_{\mathbf{k}}\right)^{2}-p^{2}-k^{2}-m_{\pi}^{2}=2 m^{2}-m_{\pi}^{2}-2 E_{\mathbf{p}} E_{\mathbf{k}} .
$$

By replacing the above integrals in Eqs. (E.1) and (E.2) we obtain

$$
\Sigma_{0}(p)=\frac{3 m f^{2}}{2 \pi^{2} m_{\pi}^{2}} \int_{0}^{k_{\mathrm{F}}} \mathrm{d} k \frac{k^{2}}{E_{\mathbf{k}}}\left[1+\frac{m_{\pi}^{2}}{4 p k} \ln \frac{\gamma(p, k)+2 p k}{\gamma(p, k)-2 p k}\right]
$$

and

$$
\alpha(p)=\frac{3 m^{2} f^{2}}{\pi^{2} E_{\mathbf{p}}} \int_{0}^{k_{\mathrm{F}}} \mathrm{d} k \frac{k^{2}}{E_{\mathbf{k}}} \cdot \frac{E_{\mathbf{k}}-E_{\mathbf{p}}}{\gamma^{2}(p, k)-4 p^{2} k^{2}} .
$$


It is interesting to note that for large $p$-values the following limits hold:

$$
\begin{aligned}
& \lim _{p \rightarrow \infty} \alpha(p)=0 \\
& \lim _{p \rightarrow \infty} \Sigma_{0}(p)=\frac{3 m f^{2}}{2 \pi^{2} m_{\pi}^{2}} \int_{0}^{k_{\mathrm{F}}} \mathrm{d} k \frac{k^{2}}{E_{\mathbf{k}}}=\frac{3 m f^{2}}{4 \pi^{2} m_{\pi}^{2}}\left(E_{\mathrm{F}} k_{\mathrm{F}}-m^{2} \ln \frac{E_{\mathrm{F}}+k_{\mathrm{F}}}{m}\right),
\end{aligned}
$$

where $E_{\mathrm{F}}=\sqrt{k_{\mathrm{F}}^{2}+m^{2}}$ is the Fermi energy. For $k_{\mathrm{F}}=237 \mathrm{MeV} / c$, the on-shell self-energy limit is $\sim 34 \mathrm{MeV}$.

\section{Appendix F. The electromagnetic current operator}

In this appendix we provide a simple derivation of the non-relativistic reduction of the singlenucleon on-shell electromagnetic current operator (see $[66,75,73]$ ). The single-nucleon electromagnetic current reads

$$
J^{\mu}\left(P^{\prime} s^{\prime} ; P s\right)=\bar{u}\left(\mathbf{p}^{\prime}, s^{\prime}\right)\left[F_{1}\left(Q^{2}\right) \gamma^{\mu}+\frac{\mathrm{i}}{2 m} F_{2}\left(Q^{2}\right) \sigma^{\mu v} Q_{v}\right] u(\mathbf{p}, s),
$$

where $P^{\mu}=(E, \mathbf{p})$ is the four-momentum of the incident nucleon, $P^{\prime \mu}=\left(E^{\prime}, \mathbf{p}^{\prime}\right)$ the four-momentum of the outgoing nucleon and $Q^{\mu}=P^{\prime \mu}-P^{\mu}=(\omega, \mathbf{q})$ the transferred four-momentum. The spin projections for incoming and outgoing nucleons are labeled $s$ and $s^{\prime}$, respectively. We follow the conventions of Bjorken and Drell [31] for the $u$-spinors. For convenience in the discussions that follow the scales in the problem we introduce the dimensionless variables: $\boldsymbol{\eta}=\mathbf{p} / m, \varepsilon=E / m=\sqrt{1+\eta^{2}}, \lambda=\omega / 2 m$, $\kappa=q / 2 m$ and $\tau=-Q^{2} / 4 m^{2}=\kappa^{2}-\lambda^{2}$. For the outgoing nucleon, $\boldsymbol{\eta}^{\prime}$ and $\varepsilon^{\prime}$ are defined correspondingly.

For any general operator whose $\Gamma$-matrix form is given by

$$
\Gamma=\left(\begin{array}{ll}
\Gamma_{11} & \Gamma_{12} \\
\Gamma_{21} & \Gamma_{22}
\end{array}\right)
$$

one has $\bar{u}\left(\mathbf{p}^{\prime}, s^{\prime}\right) \Gamma u(\mathbf{p}, s)=\chi_{s^{\prime}}^{\dagger} \bar{\Gamma} \chi_{\mathrm{s}}$, with the current operator $\bar{\Gamma}$ given by

$$
\bar{\Gamma}=\frac{1}{2} \sqrt{(1+\varepsilon)\left(1+\varepsilon^{\prime}\right)}\left(\Gamma_{11}+\Gamma_{12} \frac{\boldsymbol{\sigma} \cdot \boldsymbol{\eta}}{1+\varepsilon}-\frac{\boldsymbol{\sigma} \cdot \boldsymbol{\eta}^{\prime}}{1+\varepsilon^{\prime}} \Gamma_{21}-\frac{\boldsymbol{\sigma} \cdot \boldsymbol{\eta}^{\prime}}{1+\varepsilon^{\prime}} \Gamma_{22} \frac{\boldsymbol{\sigma} \cdot \boldsymbol{\eta}}{1+\varepsilon}\right) .
$$

An important point in our approach is that we expand only in powers of the bound nucleon momentum $\eta$, not in the transferred momentum $\kappa$ or the transferred energy $\lambda$. This is a very reasonable approximation as the momentum of the initial nucleon is relatively low in most cases, since the typical values of $\eta$ lie below $\eta_{\mathrm{F}} \equiv k_{\mathrm{F}} / m$, where $k_{\mathrm{F}}$ is the Fermi momentum $\left(\eta_{\mathrm{F}}\right.$ is typically about $\frac{1}{4}$ ). However, for those cases corresponding to short-range properties of the nuclear wave functions it will be necessary to be very careful with the approximations made. Indeed, for large values of $\eta$ a fully relativistic approach will likely prove necessary. Expanding up to first order in powers of $\eta$ we get $\varepsilon \simeq 1$ and $\varepsilon^{\prime} \simeq 1+2 \lambda$.

Thus, the non-relativistic reductions of the time and space components of the single-nucleon electromagnetic current operator can be evaluated in a rather simple form. 
Let us consider first the case of the time component. We have

$$
J^{0}\left(P^{\prime} s^{\prime} ; P s\right)=\bar{u}\left(\mathbf{p}^{\prime}, s^{\prime}\right) J^{0} u(\mathbf{p}, s)=\chi_{s^{\prime}}^{\dagger} \overline{J^{0}} \chi_{\mathrm{s}}
$$

with the current operator $J^{0}=F_{1} \gamma^{0}+\mathrm{i} F_{2} \sigma^{0 v} Q_{v} / 2 m$. Using the general result given by Eq. (F.3) and expanding up to first order in $\eta$, it is straightforward to get the relation

$$
\overline{J^{0}} \simeq \frac{\kappa}{\sqrt{\tau}} G_{\mathrm{E}}+\frac{\mathrm{i}}{\sqrt{1+\tau}}\left(G_{\mathrm{M}}-\frac{G_{\mathrm{E}}}{2}\right)(\boldsymbol{\kappa} \times \boldsymbol{\eta}) \cdot \boldsymbol{\sigma},
$$

where we have introduced the Sachs form factors $G_{\mathrm{E}}=F_{1}-\tau F_{2}$ and $G_{\mathrm{M}}=F_{1}+F_{2}$, and have used the relations

$$
\begin{aligned}
& \lambda \simeq \tau+\boldsymbol{\kappa} \cdot \boldsymbol{\eta}, \\
& \kappa^{2} \simeq \tau(1+\tau+2 \boldsymbol{\kappa} \cdot \boldsymbol{\eta}) .
\end{aligned}
$$

Expression (F.5) coincides with the leading-order expressions obtained in previous work [66,73]; in those studies a different approach was taken which, while more cumbersome, does yield terms of higher order than the ones considered in the present work. It is important to remark again that no expansions have been made in terms of the transferred energy and transferred momentum; indeed, $\kappa, \lambda$ and $\tau$ may be arbitrarily large in our approach.

Let us consider now the case of space components. Thus, we have

$$
\boldsymbol{J}\left(P^{\prime} s^{\prime} ; P s\right)=\bar{u}\left(\mathbf{p}^{\prime}, s^{\prime}\right) \boldsymbol{J} u(\mathbf{p}, s)=\chi_{s^{\prime}}^{\dagger} \overline{\boldsymbol{J}} \chi_{\mathrm{s}} .
$$

Introducing the matrix form of the vector component for the single-nucleon electromagnetic current operator in general relation (F.3), one can finally write

$$
\begin{aligned}
\overline{\boldsymbol{J}} \simeq & \frac{1}{\sqrt{1+\tau}}\left\{\mathrm{i} G_{\mathrm{M}}(\boldsymbol{\sigma} \times \boldsymbol{\kappa})+\left(G_{\mathrm{E}}+\frac{\tau}{2} G_{\mathrm{M}}\right) \boldsymbol{\eta}+G_{\mathrm{E}} \boldsymbol{\kappa}\right. \\
& -\frac{G_{\mathrm{M}}}{2(1+\tau)}(\boldsymbol{\kappa} \cdot \boldsymbol{\eta}) \boldsymbol{\kappa}-\frac{\mathrm{i} G_{\mathrm{E}}}{2(1+\tau)}(\boldsymbol{\sigma} \times \boldsymbol{\kappa}) \boldsymbol{\kappa} \cdot \boldsymbol{\eta} \\
& \left.-\mathrm{i} \tau\left(G_{\mathrm{M}}-G_{\mathrm{E}} / 2\right)(\boldsymbol{\sigma} \times \boldsymbol{\eta})+\frac{\mathrm{i}\left(G_{\mathrm{M}}-G_{\mathrm{E}}\right)}{2(1+\tau)}(\boldsymbol{\kappa} \times \boldsymbol{\eta}) \boldsymbol{\sigma} \cdot \boldsymbol{\kappa}\right\},
\end{aligned}
$$

where we have used the relations given by Eqs. (F.6) and (F.7).

In order to compare with [73], we write the expression for the transverse component of the current, i.e., $\overline{\boldsymbol{J}}^{\perp}=\overline{\boldsymbol{J}}-\left(\overline{\boldsymbol{J}} \cdot \boldsymbol{\kappa} / \kappa^{2}\right) \boldsymbol{\kappa}$. After some algebra we get the final result

$$
\begin{aligned}
\overline{\boldsymbol{J}}^{\perp} \simeq & \frac{1}{\sqrt{1+\tau}}\left\{\mathrm{i} G_{\mathrm{M}}(\boldsymbol{\sigma} \times \boldsymbol{\kappa})+\left(G_{\mathrm{E}}+\frac{\tau}{2} G_{\mathrm{M}}\right)\left(\boldsymbol{\eta}-\frac{\boldsymbol{\kappa} \cdot \boldsymbol{\eta}}{\kappa^{2}} \boldsymbol{\kappa}\right)\right. \\
& \left.-\frac{\mathrm{i} G_{\mathrm{M}}}{1+\tau}(\boldsymbol{\sigma} \times \boldsymbol{\kappa}) \boldsymbol{\kappa} \cdot \boldsymbol{\eta}+\frac{\mathrm{i} G_{\mathrm{M}}}{2(1+\tau)}(\boldsymbol{\eta} \times \boldsymbol{\kappa}) \boldsymbol{\sigma} \cdot \boldsymbol{\kappa}\right\} .
\end{aligned}
$$

It is straightforward to prove that this expression coincides with the result given by Eq. (25) in [73] for an expansion in powers of $\eta$ up to first order. 
Therefore, as can be seen from Eqs. (F.5) and (F.10), at linear order in $\eta$ we retain the spin-orbit part of the charge and one of the relativistic corrections to the transverse current, the first-order convective spin-orbit term. It is also important to remark here that the current operators given by Eqs. (F.5) and (F.9) satisfy the property of current conservation $\lambda J_{0}=\boldsymbol{\kappa} \cdot \mathbf{J}$. Finally, it is also interesting to quote the results obtained in the traditional non-relativistic reduction [73,95-98], where it is assumed that $\kappa \ll 1$ and $\lambda \ll 1$ :

$$
\begin{aligned}
& {\overline{J^{0}}}_{\text {nonrel }}=G_{\mathrm{E}}, \\
& \overline{\boldsymbol{J}}_{\text {nonrel }}^{\perp}=-\mathrm{i} G_{\mathrm{M}}[\boldsymbol{\kappa} \times \boldsymbol{\sigma}]+G_{\mathrm{E}}\left[\boldsymbol{\eta}-\left(\frac{\boldsymbol{\kappa} \cdot \boldsymbol{\eta}}{\kappa^{2}}\right) \boldsymbol{\kappa}\right] .
\end{aligned}
$$

Note that this traditional non-relativistic reduction contains both terms of zeroth and first order in $\eta$, i.e., the convection current, and is therefore not actually of lowest order in $\eta$.

We see that the expansion of the current to first order in the variable $\eta=p / m$ yields quite simple expressions; moreover, the various surviving pieces of the relativized current (i.e., charge and spin-orbit in the longitudinal and magnetization and convection in the transverse) differ from the traditional non-relativistic expressions only by multiplicative $(q, \omega)$-dependent factors such as $\kappa / \sqrt{\tau}$ or $1 / \sqrt{1+\tau}$, and therefore are easy to implement in already existing non-relativistic models.

\section{References}

[1] C.F. Williamson, et al., Phys. Rev. C 56 (1997) 3152.**

[2] V.D. Burkert, Prog. Part. Nucl. Phys. 44 (2000) 273-291.

[3] J. Arrington, et al., Phys. Rev. Lett. 82 (1999) 2056.

[4] A. Gil, J. Nieves, E. Oset, Nucl. Phys. A 627 (1997) 543. **

[5] A. Gil, J. Nieves, E. Oset, Nucl. Phys. A 627 (1997) 599.

[6] J. Carlson, J. Jourdan, R. Schiavilla, I. Sick, Phys. Rev. C 65 (2002) 024002.

[7] J. Carlson, R. Schiavilla, Rev. Mod. Phys. 70 (1998) 743. *

[8] A. Fabrocini, Phys. Rev. C 55 (1997) 338.

[9] K.S. Kim, L.E. Wright, D.A. Resler, Phys. Rev. C 64 (2001) 044607.

[10] C.J. Horowitz, B.D. Serot, Nucl. Phys. A 368 (1981) 503. *

[11] Yanhe Jin, D.S. Onley, L.E. Wright, Phys. Rev. C 45 (1992) 1311.

[12] H. Kurasawa, T. Suzuki, preprint nucl-th/0201035.

[13] P. Amore, M.B. Barbaro, A. De Pace, Phys. Rev. C 53 (1996) 2801.

[14] O. Benhar, Phys. Rev. Lett. 83 (1999) 3130.

[15] S.A. Gurvitz, S. Rinat, Phys. Rev. C 65 (2002) 024310.

[16] S. Simula, preprint nucl-th/0002030.

[17] M.W. Paris, V.R. Pandharipande, preprint nucl-th/0110048.

[18] N. Isgur, S. Jeschonnek, W. Melnitchouk, J.W. Van Orden, Phys. Rev. D 64 (2001) 054005.

[19] J.E. Amaro, M.B. Barbaro, J.A. Caballero, T.W. Donnelly, A. Molinari, Nucl. Phys. A 697 (2002) 388. ***

[20] J.E. Amaro, M.B. Barbaro, J.A. Caballero, T.W. Donnelly, A. Molinari, Nucl. Phys. A 643 (1998) 349. **

[21] J.E. Amaro, M.B. Barbaro, J.A. Caballero, T.W. Donnelly, A. Molinari, Nucl. Phys. A 657 (1999) 161.

[22] M. Chemtob, M. Rho, Nucl. Phys. A 163 (1971) 1.

[23] J.E. Amaro, A.M. Lallena, G. Co', A. Fabrocini, Phys. Rev. C 57 (1998) 3473.

[24] W.M. Alberico, T.W. Donnelly, A. Molinari, Nucl. Phys. A 512 (1990) 541. ***

[25] W.M. Alberico, A. Molinari, J. Phys. G 7 (1981) L93.

[26] J.E. Amaro, G. Co', E.M.V. Fasanelli, A.M. Lallena, Phys. Lett. B 277 (1992) 249.

[27] J.E. Amaro, G. Co', A.M. Lallena, Ann. Phys. (N.Y.) 221 (1993) 306.

[28] J.E. Amaro, G. Co', A.M. Lallena, Nucl. Phys. A 578 (1994) 365. * 
[29] P.G. Blunden, M.N. Butler, Phys. Lett. B 219 (1989) 151.

[30] C.J. Horowitz, J. Piekarewicz, Nucl. Phys. A 511 (1990) 461.

[31] J.D. Bjorken, S.D. Drell, Relativistic Quantum Mechanics, McGraw-Hill, New York, 1965.

[32] M.E. Peskin, D.V. Schroeder, An Introduction to Quantum Field Theory, Perseus, 1995.

[33] E. Schiller, H. Mutter, European Phys. J. A 11 (2001) 15.

[34] T.W. Donnelly, A.S. Raskin, Ann. Phys. (N.Y.) 169 (1986) 247. *

[35] T.W. Donnelly, M.J. Musolf, W.M. Alberico, M.B. Barbaro, A. De Pace, A. Molinari, Nucl. Phys. A 541 (1992) 525. *

[36] S. Boffi, C. Giusti, F.D. Pacati, Phys. Rep. 226 (1993) 1. **

[37] A.M. Saruis, Phys. Rep. 235 (1993) 57.

[38] S. Boffi, C. Giusti, F. Pacati, M. Radici, Electromagnetic Response of Atomic Nuclei, Oxford-Clarendon Press, Oxford, 1996.

[39] M.B. Barbaro, A. De Pace, T.W. Donnelly, A. Molinari, in: R. Cenni (Ed.), Electromagnetic Response Functions of Nuclei, Nova Science Publishers, Huntington, NY, 2001.

[40] J.E. Amaro, G. Co', A.M. Lallena, in: R. Cenni (Ed.), Electromagnetic Response Functions of Nuclei, Nova Science Publishers, Huntington, NY, 2001.

[41] L. Alvarez-Ruso, M.B. Barbaro, T.W. Donnelly, A. Molinari, Phys. Lett. B 497 (2001) 214.

[42] C.R. Chinn, A. Picklesimer, J.W. Van Orden, Phys. Rev. C 40 (1989) 790.

[43] A.L. Fetter, J.D. Walecka, Quantum Theory of Many-Particle Systems, McGraw-Hill, New York, 1971.

[44] S. Galster, et al., Nucl. Phys. B 32 (1971) 221.

[45] J.W. Van Orden, T.W. Donnelly, Ann. Phys. 131 (1981) 451. ***

[46] F. Gross, D.O. Riska, Phys. Rev. C 36 (1987) 1928.

[47] J.C. Ward, Phys. Rev. 78 (1950) 182.

[48] Y. Takahashi, Nuov. Cim. 6 (1957) 371.

[49] L.S. Celenza, C.M. Shakin, Relativistic Nuclear Physics, World Scientific, Singapore, 1986. *

[50] J.E. Amaro, M.B. Barbaro, J.A. Caballero, T.W. Donnelly, A. Molinari, in preparation.

[51] W.M. Alberico, M.B. Barbaro, A. De Pace, T.W. Donnelly, A. Molinari, Nucl. Phys. A 563 (1993) 605.

[52] J. Dubach, J.H. Koch, T.W. Donnelly, Nucl. Phys. A 271 (1976) 279.

[53] D.O. Riska, Phys. Rep. 181 (1989) 208.

[54] J.F. Mathiot, Phys. Rep. 173 (1989) 64.

[55] M.B. Barbaro, A. De Pace, T.W. Donnelly, A. Molinari, Nucl. Phys. A 569 (1994) 701.

[56] J.E. Amaro, B. Ameziane, A.M. Lallena, Phys. Rev. C 53 (1996) 1430.

[57] P.J. Barneo, J.E. Amaro, A.M. Lallena, Phys. Rev. C 60 (1999) 044615.

[58] J.E. Amaro, A.M. Lallena, G. Co', Ann. Phys. (N.Y.) 221 (1993) 306.

[59] J.E. Amaro, Ph.D. Thesis, Granada 1993, unpublished.

[60] T.W. Donnelly, I. Sick, Phys. Rev. Lett. 82 (1999) 3212.

[61] T.W. Donnelly, I. Sick, Phys. Rev. C 60 (1999) 065502.

[62] C. Maieron, T.W. Donnelly, I. Sick, Phys. Rev. C 65 (2002) 025502.

[63] M.R. Anastasio, L.S. Celenza, C.M. Shakin, Phys. Rev. 23 (1981) 569.

[64] M.J. Musolf, T.W. Donnelly, J. Dubach, S.J. Pollock, S. Kowalski, E.J. Beise, Phys. Rep. 239 (1994) 1. ***

[65] M. Rekalo, J. Arvieux, E. Tomasi-Gustafsson, Phys. Rev. C 65 (2002) 035501.

[66] J.E. Amaro, J.A. Caballero, E. Moya de Guerra, T.W. Donnelly, A.M. Lallena, J.M. Udias, Nucl. Phys. A 602 (1996) 263.*

[67] M.B. Barbaro, A. De Pace, T.W. Donnelly, A. Molinari, Nucl. Phys. A 598 (1996) 503.

[68] M. Kohno, N. Ohtsuka, Phys. Lett. B 98 (1981) 335.

[69] S. Boffi, M. Radici, Nucl. Phys. A 526 (1991) 602.

[70] V. Van der Sluys, J. Ryckebusch, M. Waroquier, Phys. Rev. C 49 (1994) 2695.

[71] V. Van der Sluys, J. Ryckebusch, M. Waroquier, Phys. Rev. C 51 (1995) 2664.

[72] J.E. Amaro, A.M. Lallena, J.A. Caballero, Phys. Rev. C 60 (1999) 014602.

[73] S. Jeschonnek, T.W. Donnelly, Phys. Rev. C 57 (1998) 2438.

[74] M. Mazziotta, J.E. Amaro, F. Arias de Saavedra, Phys. Rev. C 65 (2002) 034602.

[75] J.E. Amaro, J.A. Caballero, E. Moya de Guerra, T.W. Donnelly, Nucl. Phys. A 611 (1996) 163. 
[76] J.E. Amaro, T.W. Donnelly, Ann. Phys. (N.Y.) 263 (1998) 56.

[77] J.E. Amaro, T.W. Donnelly, Nucl. Phys. A 646 (1999) 187.

[78] J.E. Amaro, T.W. Donnelly, Nucl. Phys. A 703 (2002) 541.

[79] J.M. Udias, J.A. Caballero, E. Moya de Guerra, J.E. Amaro, T.W. Donnelly, Phys. Rev. Lett. 83 (1999) 5451.

[80] J.A. Caballero, M.C. Martinez, E. Moya de Guerra, J.M. Udias, J.E. Amaro, T.W. Donnelly, Nucl. Phys. A 689 (2001) 449c.

[81] M.C. Martínez, J.A. Caballero, T.W. Donnelly, nucl-th/0201004, Nucl. Phys. A, in press.

[82] J.E. Amaro, G. Co', A.M. Lallena, Int. J. Mod. Phys. E 3 (1994) 735.

[83] T. deForest, J.D. Walecka, Adv. Phys. 15 (1966) 1.

[84] J.L. Friar, Ann. Phys. (N.Y.) 81 (1973) 332.

[85] C. Ciofi degli Atti, Prog. Part. Nucl. Phys. 3 (1980) 163.

[86] W.M. Alberico, A. Molinari, T.W. Donnelly, E.L. Kronenberg, J.W. Van Orden, Phys. Rev. C 38 (1988) 1801.

[87] B.D. Serot, J.D. Walecka, Adv. Nucl. Phys. 16 (1986) 1.

[88] M.J. Dekker, P.J. Brussaard, J.A. Tjon, Phys. Lett. B 289 (1992) 255.

[89] M.J. Dekker, P.J. Brussaard, J.A. Tjon, Phys. Rev. C 49 (1994) 2650.

[90] G. Orlandini, M. Traini, Rep. Prog. Phys. 54 (1991) 257.

[91] J. Jourdan, Phys. Lett. B 353 (1995) 189.

[92] J. Jourdan, Nucl. Phys. A 603 (1996) 117.

[93] R. Cenni, T.W. Donnelly, A. Molinari, Phys. Rev. C 56 (1997) 276.

[94] R. Cenni, Nucl. Phys. A 696 (2001) 605.

[95] S. Boffi, C. Giusti, F.D. Pacati, Nucl. Phys. A 336 (1980) 416.

[96] C. Giusti, F.D. Pacati, Nucl. Phys. A 336 (1980) 427.

[97] J. Adam Jr., H. Arenhövel, Nucl. Phys. A 614 (1997) 289.

[98] F. Ritz, H. Göller, T. Wilbois, H. Arenhövel, Phys. Rev. C 55 (1997) 2214. 